\title{
Cyclic Hypervalent lodine Reagents for Azidation: Safer Reagents and Photoredox-Catalyzed Ring Expansion
}

\author{
Sebastien Alazet, ${ }^{\dagger, \perp}$ Johannes Preindl, ${ }^{\dagger, \perp}$ Raphael Simonet-Davin, ${ }^{\dagger}$ Stefano Nicolai, ${ }^{\dagger}$ Annik Nanchen, ${ }^{\dagger}$ \\ Thierry Meyer, ${ }^{\S \odot}$ and Jerome Waser* ${ }^{*} \odot \odot$ \\ ${ }^{\dagger}$ Laboratory of Catalysis and Organic Synthesis, Institute of Chemical Sciences and Engineering, Ecole Polytechnique Fédérale de \\ Lausanne, EPFL SB ISIC LCSO, BCH 4306, CH-1015 Lausanne, Switzerland \\ ${ }^{\ddagger}$ Process Safety, TÜV SÜD Schweiz AG, CH-4002 Basel, Switzerland \\ ${ }^{\S}$ Group of Chemical and Physical Safety, Institute of Chemical Sciences and Engineering, Ecole Polytechnique Fédérale de \\ Lausanne, CH-1015 Lausanne, Switzerland
}

Supporting Information

ABSTRACT: Azides are building blocks of increasing importance in synthetic chemistry, chemical biology, and materials science. Azidobenziodoxolone ( $\mathrm{ABX}$, Zhdankin reagent) is a valuable azide source, but its safety profile has not been thoroughly established. Herein, we report a safety study of $A B X$, which shows its hazardous nature. We introduce two derivatives, $t \mathrm{Bu}-\mathrm{ABX}$ and $\mathrm{ABZ}$ (azidobenziodazolone), with a better safety profile, and use them in established photoredox- and metal-mediated
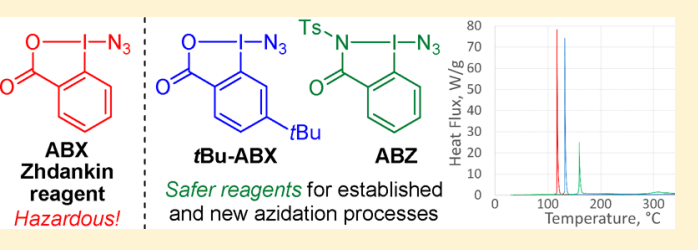

azidations, and in a new ring-expansion of silylated cyclobutanols to give azidated cyclopentanones.

rganic azides are versatile building blocks in synthetic chemistry, chemical biology, and materials science. ${ }^{1}$ Therefore, the development of a new synthetic methodology to access them is an important field of research. ${ }^{2}$ In this context, azide-containing hypervalent iodine(III) reagents $^{3}$ derived from iodobenzene have been known for a long time as sources of electrophilic azides or azido radicals. ${ }^{4}$ Nevertheless, these reagents are highly unstable at room temperature and need to be generated in situ.

In 1994, an important progress was realized by Zhdankin and co-workers, ${ }^{5}$ shortly followed by Kita and co-workers, ${ }^{6}$ with the isolation of the first cyclic hypervalent iodine reagents being stable up to $100{ }^{\circ} \mathrm{C}$ : the azidobenziodoxol(on)es (ABX) 1a-1c (Scheme 1A). Zhdankin demonstrated the functionalization of $\mathrm{C}-\mathrm{H}$ bonds with azidobenziodoxolone 1a (Zhdankin reagent) under mild thermal activation with or without dibenzoyl peroxide as the initiator. ${ }^{5 c}$ Surprisingly, this result did not attract the attention it should have, and it was only in 2013 that our group, as well as Gade and co-workers, reported the excellent properties of $A B X$ reagents as a source of electrophilic azides, whereas Studer and co-workers reported reductive conditions to generate azido radicals from $1 \mathbf{1 a}^{7}$ Since then, the Zhdankin reagent 1a has established itself as an excellent azide source, either under thermal, ${ }^{5 c, 8}$ photoredox, ${ }^{9}$ or metal-mediated activation. ${ }^{10}$

In 2017, our group reported the divergent reactivity of the Zhdankin reagent (1a) and azidobenziodoxole $\mathbf{1 b}$ in the azidolactonization of alkenes (Scheme 1B). ${ }^{11}$ Whereas 1a was ideally suited for 1,2-azidation under photoredox conditions, Lewis acid activation of $\mathbf{1 b}$ led to 1,1-azidolactonization via an 1,2-aryl shift. On the basis of these results, we wondered if we could also develop an azidative ring-expansion of alkene-substituted
Scheme 1. Cyclic Hypervalent Iodine Reagents as Azide Sources

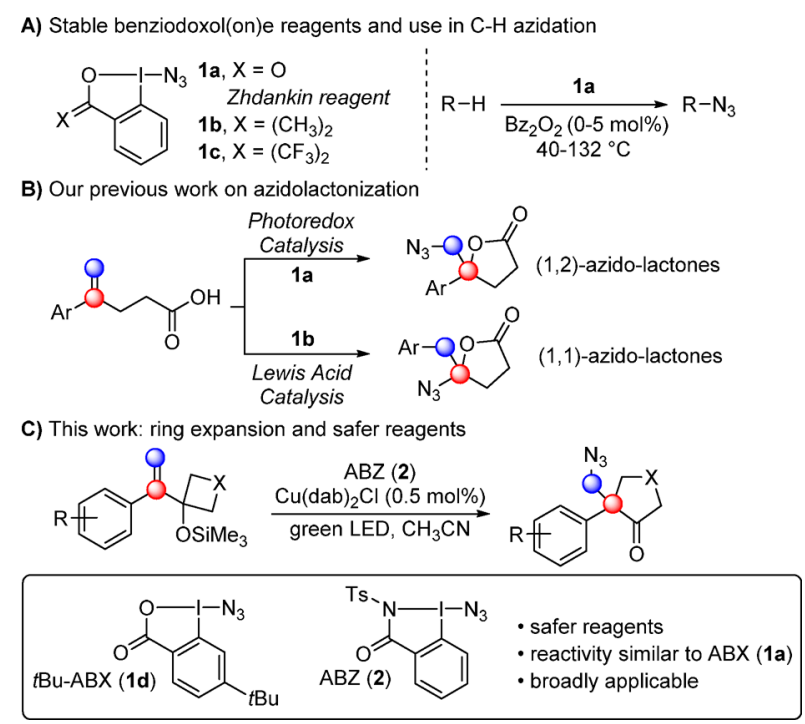

cyclobutanol derivatives via reaction with azido radicals (Scheme 1C). Such ring expansions are usually initiated by the addition of electrophilic ${ }^{12}$ or organometallic ${ }^{13}$ intermedi- $^{-}$ ates onto the olefin. More recently, the addition of a radical followed by oxidation and an 1,2-shift under photoredox conditions has emerged as a very efficient approach for ring

Received: August 17, 2018

Published: September 15, 2018 
expansion, ${ }^{14}$ but it has never been reported for the synthesis of organic azides. ${ }^{15}$ Preliminary results with the Zhdankin reagent (1a) using a copper photoredox catalyst were highly promising, but a spontaneous explosion of a pure sample of 1a in our laboratory made us aware of serious safety hazards.

Herein, we report safety studies of the Zhdankin reagent (1a), which highlighted a very high shock and friction sensitivity. ${ }^{16}$ The stability of two higher weight derivatives ( $t \mathrm{Bu}$ $A B X$ (1c) and $A B Z$ (azidobenziodazolone 2), Scheme 1C) was investigated, showing an enhanced safety profile. $A B Z$ (2), in particular, was well suited for the generation of azido radicals under mild photoredox conditions, allowing us to develop the desired ring expansion in high yield and with a broad scope. Finally, we demonstrate that either $t \mathrm{Bu}-\mathrm{ABX}$ (1d) or ABZ (2) can be used as alternatives to the Zhdankin reagent in a broad range of transformations involving thermal, photoredox, or metal-mediated activation.

On the basis of our photocatalyzed azidolactonisation work, ${ }^{11}$ we started our investigations on the tandem azidation-ring expansion by reacting 1-(1-phenylvinyl)cyclobutanol (3a) with $\mathrm{ABX}$ (1a) (2.0 equiv) as an azide precursor in acetonitrile in the presence of $\mathrm{Cu}(\mathrm{dap})_{2} \mathrm{Cl}(5)^{17}(0.5-1 \mathrm{~mol} \%)$ under blue LED irradiation (eq 1). The expected product 6a was obtained in
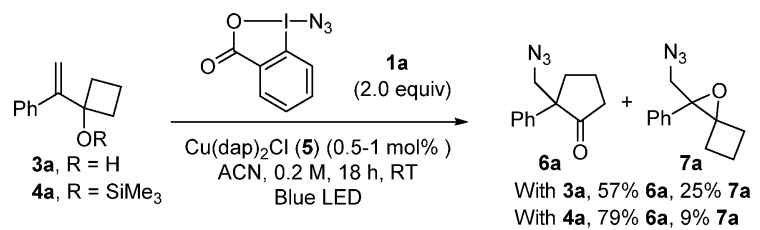

$57 \%$ yield as a mixture with $25 \%$ of 1,2 -azido-epoxyde $7 \mathrm{a}$. To avoid this oxyazidation process, the silyl-protected precursor 4a was used, and the ring-expansion product $\mathbf{6 a}$ could be obtained in an improved $79 \%$ yield.

However, during further optimization studies, a freshly prepared batch of $A B X$ reagent (1a) (500 mg) spontaneously exploded when the researcher was handling the reagent with a spatula in a glass flask, causing multiple serious cuts and burns. ${ }^{18}$ We therefore decided to stop experimentation with reagent 1a until its safety profile had been further evaluated. In addition, we synthesized the azidobenziodoxolone reagents $t \mathrm{Bu}-\mathrm{ABX}(1 \mathrm{~d})$ and $\mathrm{ABZ}$ (2) with higher molecular weight, hoping that the lower proportional azide content would diminish their explosion potential (Scheme 1C). Both reagents 1d and $\mathbf{2}$ are easy accessible on gram scale from 4-tert-butyl toluene (3.4 $\mathrm{g}$ in 5 steps and $31 \%$ overall yield) and 2-iodobenzoic acid (5.4 g in 4 steps and $30 \%$ overall yield), respectively.

The stability of the compounds was then determined by differential scanning calorimetry (DSC, Figure 1 and Table 1). ${ }^{19}$ According to Stoessel, ${ }^{20}$ thermal risk is the combination of the severity (expressed by the average heat release) and the likelihood of occurrence (defined as $\mathrm{TD}_{24}$ corresponding to the temperature at which the time to the maximum rate of the decomposition reaction is $24 \mathrm{~h}$ ). Mage et al. ${ }^{21}$ defined a $3 \times 3$ risk matrix using both the released energy and the $\mathrm{TD}_{24}$. Compounds with severity higher than $400 \mathrm{~kJ} / \mathrm{kg}$ and $\mathrm{TD}_{24}$ below $150{ }^{\circ} \mathrm{C}$ are classified in the most hazardous category. The DSC results (see Table 1) revealed a heat release of $1770 \mathrm{~kJ} / \mathrm{kg}$ for the Zhdankin reagent (1a), $1440 \mathrm{~kJ} / \mathrm{kg}$ for $t \mathrm{Bu}-\mathrm{ABX}$ (1d), and $965 \mathrm{~kJ} / \mathrm{kg}$ (first peak, with a second peak of $380 \mathrm{~kJ} / \mathrm{kg}$ ) for ABZ (2). Onset temperatures were higher for $\mathbf{1 d}$ and 2. Estimated values ${ }^{22}$ for $\mathrm{TD}_{24}$ are $45{ }^{\circ} \mathrm{C}$ for the Zhdankin

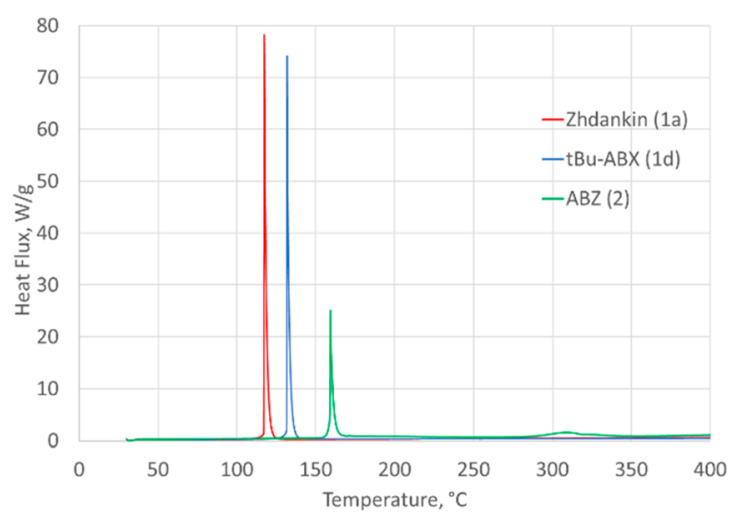

Figure 1. DSC thermograms for the three studied reagents.

reagent (1a), $52{ }^{\circ} \mathrm{C}$ for $t \mathrm{Bu}-\mathrm{ABX}$ (1d), and $88^{\circ} \mathrm{C}$ for $\mathrm{ABZ}$ (2). Therefore, all three compounds have a high severity and likelihood of occurrence, with the Zhdankin reagent having the highest thermal potential hazard.

Another safety parameter for these compounds is related to impact sensitivity. A Fallhammer test showed limiting energies of 1,2 , and $5 \mathrm{~J}$ for reagents $\mathbf{1 a}, \mathbf{1 d}$, and $\mathbf{2}$ (Table 1). As a comparison, the energy released by the fall of a flask on the floor can be estimated to $3.4 \mathrm{~J}$ and of a spatula in a flask to $0.1 \mathrm{~J} .{ }^{23}$ Both scenarios are hazardous for the Zhdankin reagent (1a), even if the lower limit for impact could not be determined experimentally. In addition, all three reagents were sensitive to a friction load of $360 \mathrm{~N}$ on a porcelain plate. The results for $\mathrm{ABZ}(\mathbf{2})$ and $t \mathrm{Bu}-\mathrm{ABX}$ (1d) were, however, most probably false positives as they showed no friction sensitivity on Alox plates.

In light of the preliminary safety studies, $\mathrm{ABZ}(2)$ is the most stable reagent to use as an azide source.

Motivated by the better safety profiles of $t \mathrm{Bu}-\mathrm{ABX}(\mathbf{1 d})$ and, especially, $A B Z$ (2), we then investigated the use of these reagents in the ring expansion reaction. The best results were obtained with $\mathrm{ABZ}$ (2), and the desired product 6a was obtained in $90 \%$ yield after a short optimization (Scheme 2). ${ }^{24}$ This is an important result, demonstrating that Zhdankin and $\mathrm{ABZ}$ reagents $\mathbf{1 a}$ and $\mathbf{2}$ have similar efficiency in the generation of azido radicals under photoredox conditions. The scope of the transformation was broad, and para-substituted styrenes with alkyl-, phenyl-, as well as methoxy-, and chlorine- groups gave the desired cyclopentanones $\mathbf{6 a - 6 g}$ in excellent yields. Cyclopentanones $\mathbf{6 h}-\mathbf{j}$ and $6 \mathbf{k}$ with either a meta or an ortho substituent on the arene ring were also obtained in very good yields. Meta, para-disubstituted derivatives 61-6n were as well isolated in very good yields. 1-Naphthyl substituted 6o was isolated in $95 \%$ yield, whereas the more hindered 2-naphthyl derivative $6 \mathrm{p}$ was obtained in $79 \%$ yield. Interestingly, oxetanes were suitable substrates for the ring expansion reaction, and 3 -furanone $6 \mathrm{q}$ was obtained in $58 \%$ yield. However, the corresponding Boc-protected azetidine did not react. Styrenes with 1,2 disubstituted olefins were also not suitable substrates, and the secondary azide 6s was not obtained. A surprising result was observed when the para- $\mathrm{CF}_{3}$-substituted styrene $4 \mathrm{t}$ was subjected to the optimized reaction conditions: epoxide $7 \mathbf{t}$ was isolated as the only product. Azides are versatile functional groups, and the obtained products were readily converted into useful structural motives, such as triazoles, protected amines, or isothiocyanates.

When considering the better safety profile of ABZ (2) and to a lesser extend $t \mathrm{Bu}-\mathrm{ABX}(\mathbf{1 d})$, we decided to investigate their 
Table 1. Safety Test Results

\begin{tabular}{|c|c|c|c|c|c|}
\hline test & data & units & 1a & 1d & 2 \\
\hline \multirow[t]{3}{*}{ DSC } & decomposition energy & $\mathrm{kJ} / \mathrm{kg}$ & 1770 & 1440 & $965^{b}, 380^{c}$ \\
\hline & $T_{\text {onset }}$ & ${ }^{\circ} \mathrm{C}$ & 90 & $98-110^{a}$ & $143^{b}, 268^{c}$ \\
\hline & $T_{\text {peak }}$ & ${ }^{\circ} \mathrm{C}$ & 127 & 139 & $159^{b}, 308^{c}$ \\
\hline impact & limiting energy & $\mathrm{J}$ & $<1$ & 2 & 5 \\
\hline \multirow[t]{2}{*}{ friction } & friction load $360 \mathrm{~N}$ porcelain plate & & positive & positive & positive \\
\hline & friction load $360 \mathrm{~N}$ alox plate & & positive & negative & negative \\
\hline
\end{tabular}

${ }^{a}$ Large variation in onset temperature between two measurements. ${ }^{b}$ First peak. ${ }^{c}$ Second peak.

Scheme 2. Scope of the Ring Expansion Reaction

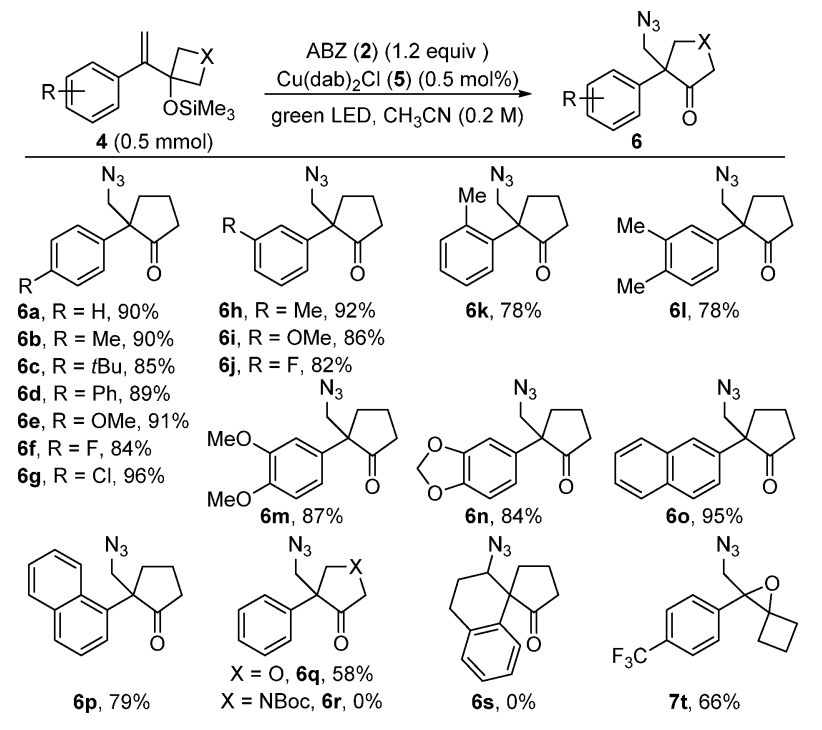

use in other transformations recently discovered using $\mathrm{ABX}$ (1a). First, we examined the azidolactonisation previously reported in our group (eq 2). ${ }^{11}$ The reaction of alkene 8 gave $76 \%$ yield
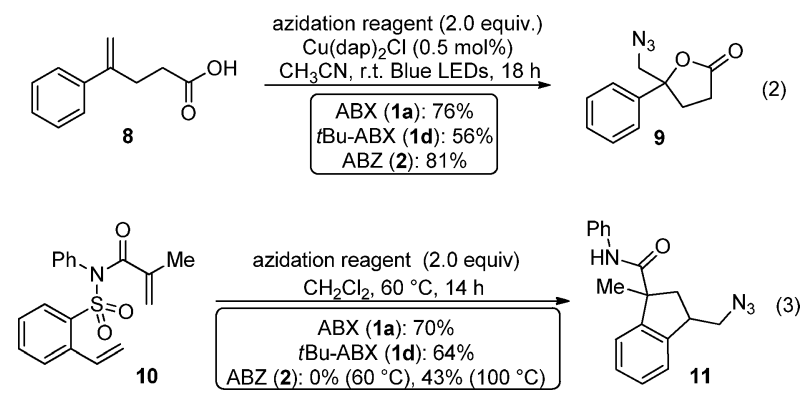

of azidolactone 9 with $\mathrm{ABX}$ (1a), 56\% with $t \mathrm{Bu}-\mathrm{ABX}$ (1d), and $81 \%$ with $\mathrm{ABZ}$ (2). This result definitively confirmed that $\mathrm{ABZ}$ (2) is an excellent and safer substitute of $\mathrm{ABX}$ (1a) for the generation of azide radicals under photoredox conditions. Another azidative cyclization, but using thermal activation, was then investigated (eq 2). The cyclization of $\mathbf{1 0}$ to give azide 11 has been reported in $70 \%$ yield with $\mathrm{ABX}$ (1a) by the Nevado group. ${ }^{8 \mathrm{C}} t \mathrm{Bu}-\mathrm{ABX}$ (1d) gave $64 \%$ yield of $\mathbf{1 1}$, but $\mathrm{ABZ}$ did not show any reactivity at $60{ }^{\circ} \mathrm{C}$. However, by heating at $100{ }^{\circ} \mathrm{C}, \mathbf{1 1}$ could be obtained in $43 \%$ yield with $\mathrm{ABZ}$ (2). This is in good accordance with the higher thermal stability of $\mathrm{ABZ}$ (2), requiring higher temperature for activation.

Next, metal-catalyzed azidation processes were examined, starting with the iron-catalyzed $\mathrm{sp}^{3} \mathrm{C}-\mathrm{H}$ azidation reported by Hartwig and co-workers (eq 4). ${ }^{10 c}$ The representative reaction selected was the azidation of cumene (12). Compared to

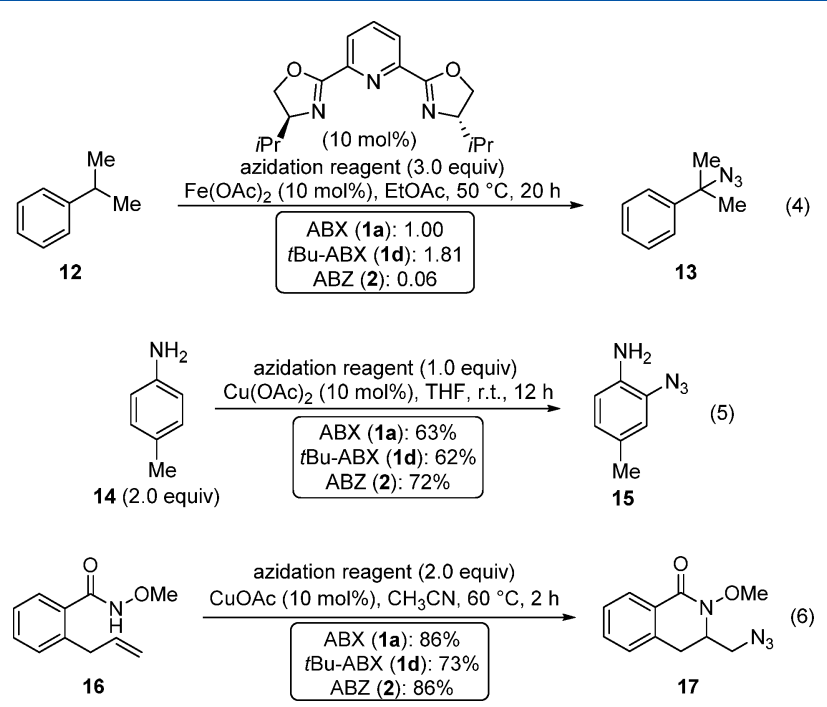

$\mathrm{ABX}(1 \mathrm{a})$ set at 1.00 , relative yields of 1.81 and 0.06 were obtained for $t \mathrm{Bu}-\mathrm{ABX}(1 \mathrm{~d})$ and $\mathrm{ABZ}$ (2), respectively, by GC-MS. ${ }^{25}$ This result may be due to the relative solubility of the reagents in ethyl acetate: $A B Z$ (2) was nearly insoluble, while $t \mathrm{Bu}-\mathrm{ABX}$ (1d) was more soluble than $\mathrm{ABX}$ (1a). As a next example, we examined the copper-catalyzed $\mathrm{SP}^{2} \mathrm{C}-\mathrm{H}$ bond azidation of anilines reported by Hao and co-workers (eq 5). ${ }^{10 b}$ Azide 15 was obtained in 63,62 , or $72 \%$ yields ${ }^{26}$ using reagents 1a, 1d, or 2, respectively, from $p$-toluidine (14). Finally, the copper-catalyzed aminoazidation of olefins developed by Wang and co-workers was examined on alkene 16 (eq 6). ${ }^{10 \mathrm{~h}}$ Again, no significant difference of reactivity between the reagents was observed, and the use of ABZ (2) should be favored.

In summary, we have reported the first in-depth safety studies of the hypervalent iodine azidation reagents $A B X$ (Zhdankin reagent, 1a), $t \mathrm{Bu}-\mathrm{ABX}$ (1d), and $\mathrm{ABZ}$ (2), showing the better profile of the latest in particular. $A B Z$ (2) was as efficient as the Zhdankin reagent (1a) in a new photoredoxmediated ring expansion process as well as in established radical- or metal-mediated transformations. When considering the growing importance of hypervalent iodine reagents for azide transfer in synthetic chemistry, we are convinced that our work will be highly useful for researchers to continue to exploit their amazing reactivity in safer settings.

\section{EXPERIMENTAL SECTION}

General Methods. All reactions were carried out in oven- or flame-dried glassware under a nitrogen atmosphere, unless stated otherwise. For quantitative flash chromatography, distilled technical grade solvents were used. THF, $\mathrm{Et}_{2} \mathrm{O}, \mathrm{CH}_{3} \mathrm{CN}$, and $\mathrm{CH}_{2} \mathrm{Cl}_{2}$ were dried by passage over activated alumina under a nitrogen atmosphere $\left(\mathrm{H}_{2} \mathrm{O}\right.$ content $<7 \mathrm{ppm}$, Karl Fischer titration). $\mathrm{NEt}_{3}$ was dried by distillation over $\mathrm{CaH}_{2}$ under a nitrogen atmosphere. All chemicals were purchased and used as received, unless stated otherwise. 
Chromatographic purification was performed as flash chromatography using Macherey-Nagel silica 40-63, $60 \AA$, using the solvents indicated as the eluent with $0.1-0.5$ bar pressure. TLC was performed on Merck silica gel 60 F254 TLC aluminum plates and visualized with UV-light, permanganate, CAM, or $p$-anisaldehyde stains. ${ }^{1} \mathrm{H}$ NMR spectra were recorded at room temperature on a Bruker DPX-400 $400 \mathrm{MHz}$ spectrometer in chloroform- $d$ or $d_{6}$-DMSO; all signals are reported in ppm with the internal chloroform signal at $7.26 \mathrm{ppm}$ or the internal $d_{6}$-DMSO signal at $2.50 \mathrm{ppm}$ as the standard. The data are being reported as $(\mathrm{s}=$ singlet, $\mathrm{d}=$ doublet, $\mathrm{t}=$ triplet, $\mathrm{q}=$ quadruplet, $\mathrm{qi}=$ quintet, $\mathrm{m}=$ multiplet or unresolved, $\mathrm{br}=$ broad signal, integration, coupling constant(s) in $\mathrm{Hz}$, interpretation). ${ }^{13} \mathrm{C}$ NMR spectra were recorded with ${ }^{1} \mathrm{H}$-decoupling on a Bruker DPX-400 $101 \mathrm{MHz}$ spectrometer in chloroform- $d$ or $d_{6}$-DMSO; all signals are reported in ppm with the internal chloroform signal at $77.00 \mathrm{ppm}$ or the internal DMSO signal at $39.51 \mathrm{ppm}$ as the standard. Infrared spectra were recorded on a JASCO FT-IR B4100 spectrophotometer with an ATR PRO4100-S and a ZnSe prism and are reported as $\mathrm{cm}^{-1}$ ( $\mathrm{w}=$ weak, $\mathrm{m}=$ medium, $\mathrm{s}=$ strong $)$. High-resolution mass spectrometric measurements were performed by the mass spectrometry service of ISIC at the EPFL on a MICROMASS (ESI): Q-TOF Ultima API. Melting points were measured on a Buechi B-540 melting point apparatus and were not corrected. Reactions were performed in test tubes $(1.0-0 \mathrm{~mL})$, which were held using a rack for test tubes placed at the center of a crystallization flask. On this flask, were attached the LEDs (LED ribbon cable with open ends, Barthelme Y51516414 $18240524 \mathrm{~V} 502 \mathrm{~cm}$ blue $1 \mathrm{pc}(\mathrm{s})$, and LED ribbon cable with open ends, Barthelme Y51515213 18200712 V $502 \mathrm{~cm}$ green $1 \mathrm{pc}(\mathrm{s})$, both purchased directly on www.conrad.ch/fr). The distance between the LEDs and the test tubes was approximately $3-4 \mathrm{~cm}$. Long irradiation for more than $2 \mathrm{~h}$ resulted in the temperature increasing up to $34{ }^{\circ} \mathrm{C}$. 3GC-MS analysis was performed on a TSQ 8000 EVO coupled with TRACE 1300 through a Sebron ZB-5 ms column. The injection volume was $1 \mu \mathrm{L}$ at $250{ }^{\circ} \mathrm{C}$, and the gradient started at $50{ }^{\circ} \mathrm{C}$ for $3 \mathrm{~min}$, then $15^{\circ} \mathrm{C} /$ mins up to $300^{\circ} \mathrm{C}$, and finally $300{ }^{\circ} \mathrm{C}$ for $10 \mathrm{~min}$. Reactions under microwave irradiation were performed using a Biotage Initiator reactor in a sealed vial using external temperature control.

Synthesis of $A B X(1 \mathrm{a})$. Caution: For safety reasons, the reaction was carried out behind an antiblast shield. Following a reported procedure, $^{7 \mathrm{~b}}$ 2-iodobenzoic acid (18) (13.6 g, $54.8 \mathrm{mmol}, 1.0$ equiv) and $\mathrm{NaIO}_{4}(17 \mathrm{~g}, 81 \mathrm{mmol}, 1.0$ equiv) were suspended in aq. $\mathrm{AcOH}$ $(30 \% \mathrm{v} / \mathrm{v}, 83 \mathrm{~mL})$. The mixture was stirred at reflux $\left(120^{\circ} \mathrm{C}\right)$ for $4 \mathrm{~h}$. Past this time, ice-cold water $(80 \mathrm{~mL})$ was added under stirring, and the mixture was allowed to cool down to room temperature, while being protected from light with aluminum foil. It was then filtered, and the solid was washed with ice-cold water $(3 \times 70 \mathrm{~mL})$ and cold acetone $(3 \times 70 \mathrm{~mL})$. The resulting colorless solid (HO-BX, $13.4 \mathrm{~g}$, $50.6 \mathrm{mmol}, 92 \%$ yield) was allowed to dry in the air overnight and then directly used in the next step. ${ }^{1} \mathrm{H}$ NMR $\left(400 \mathrm{MHz}, d_{6}\right.$-DMSO, $\delta): 8.02(\mathrm{dd}, J=7.7,1.4 \mathrm{~Hz}, 1 \mathrm{H}), 7.97(\mathrm{~m}, 1 \mathrm{H}), 7.85(\mathrm{dd}, J=8.2$, $0.7 \mathrm{~Hz}, 1 \mathrm{H}), 7.71(\mathrm{td}, J=7.6,1.2 \mathrm{~Hz}, 1 \mathrm{H}) \mathrm{ppm} .{ }^{13} \mathrm{C}\left\{{ }^{1} \mathrm{H}\right\}$ NMR $\left(100 \mathrm{MHz}, d_{6}\right.$-DMSO, $\left.\delta\right): 167.7,134.5,131.5,131.1,130.4,126.3$, $120.4 \mathrm{ppm}$. The signals of the NMR spectra were in accordance with the data reported in the literature. ${ }^{7 \mathrm{~b}}$

Caution: For safety reasons, the reaction was carried out behind an antiblast shield. Following a reported procedure, ${ }^{7 \mathrm{~b}}$ HO-BX (5.70 g, $21.6 \mathrm{mmol})$ was suspended in acetic anhydride $(20 \mathrm{~mL})$. The suspension was stirred to reflux $\left(140{ }^{\circ} \mathrm{C}\right)$, until it converted into a clear solution. Heating was then stopped, and the solution was allowed to cool down to room temperature over a period of $1.5 \mathrm{~h}$, resulting in the precipitation of a crystalline, colorless solid. Crystallization was continued at $-18{ }^{\circ} \mathrm{C}$ overnight. The solid was collected by filtration and washed with several portions of pentane. Pure AcO-BX (5.50 g, $18.0 \mathrm{mmol}, 83 \%$ yield) was dried in the air overnight and directly used as such. ${ }^{1} \mathrm{H}$ NMR $\left(400 \mathrm{MHz}, \mathrm{CDCl}_{3}, \delta\right): 8.25(\mathrm{dd} J=7.6,1.4 \mathrm{~Hz}$, $1 \mathrm{H}), 8.00(\mathrm{dd}, J=8.3,0.5 \mathrm{~Hz}, 1 \mathrm{H}), 7.92(\mathrm{dt}, J=7.0,1.7 \mathrm{~Hz}, 1 \mathrm{H})$, $7.71(\mathrm{td}, J=7.6,0.9 \mathrm{~Hz}, 1 \mathrm{H}), 2.25(\mathrm{~s}, 3 \mathrm{H}) \mathrm{ppm}$. The signals of the the ${ }^{1} \mathrm{H}$ NMR spectra were in accordance with the data reported in the literature. $^{7 \mathrm{~b}}$
Caution: For safety reasons, the reaction was carried out behind an antiblast shield. However, this procedure led to occasional detonations, one of which with severe injuries (see Supporting Information). Following a reported procedure, ${ }^{7 \mathrm{~b}}$ AcO-BX (1.00 g, $3.28 \mathrm{mmol}, 1.00$ equiv, synthesized in our laboratory) was stirred in dry DCM $(3 \mathrm{~mL})$, and then $\mathrm{TMSN}_{3}(0.66 \mathrm{~mL}, 4.9 \mathrm{mmol}, 1.5$ equiv, 94\% from Alfa Aesar, catalogue number L00173-22) was cautiously added. A catalytic amount of TMSOTf $(3 \mu \mathrm{L}, 0.02 \mathrm{mmol}, 0.005$ equiv, Fluorochem, catalogue number S20400-250g) was added last to the mixture, which was then stirred at room temperature for $30 \mathrm{~min}$. The solvent was then removed under reduced pressure at room temperature, and the residue was dried under high vacuum for $1 \mathrm{~h}$ to give a yellow solid, which was washed with pentane $(2 \times 10 \mathrm{~mL})$, cold acetone $(2 \times 5 \mathrm{~mL})$, and pentane $(2 \times 10 \mathrm{~mL})$ and dried $1 \mathrm{~h}$ under a high vacuum. ABX (1a) (0.711 g, $2.46 \mathrm{mmol}, 75 \%$ yield) was obtained as a pale yellow solid.

Caution: For safety reasons, the reaction was carried out behind an antiblast shield. Following a modified reported procedure, ${ }^{7 \mathrm{~b}}$ AcO-BX $(306 \mathrm{mg}, 1.00 \mathrm{mmol}, 1.0$ equiv) was dissolved in dry DCM $(2 \mathrm{~mL})$. TMS-azide $(0.21 \mathrm{~mL}, 1.5 \mathrm{mmol}, 1.5$ equiv) was cautiously added by syringe dropwise at $0{ }^{\circ} \mathrm{C}$, leading to the conversion of the initial colorless mixture to a yellowish suspension. One drop of TMS-triflate (ca. $0.90 \mu \mathrm{L}, 5.0 \mathrm{mmol}, 5 \mathrm{~mol} \%$ ) was finally added, and the mixture was stirred at $0{ }^{\circ} \mathrm{C}$ for $30 \mathrm{~min}$. The solids were then filtered under low pressure suction in two portions (for safety reasons), washed with pentane, and dried in the air for $15 \mathrm{~min} . \mathrm{ABX}(1 \mathrm{a})(0.246 \mathrm{~g}, 0.853 \mathrm{mmol}$, $85 \%$ yield) was obtained as a pale yellow solid. ${ }^{1} \mathrm{H}$ NMR $(400 \mathrm{MHz}$, $\left.\mathrm{CDCl}_{3}, \delta\right): 8.19(\mathrm{dd}, J=7.5,1.4 \mathrm{~Hz}, 1 \mathrm{H}), 7.95(\mathrm{dd}, J=8.4,1.3 \mathrm{~Hz}$, $1 \mathrm{H}$ ), 7.91 (ddd, $J=8.4,7.0,1.4 \mathrm{~Hz}, 1 \mathrm{H}$ ), 7.70 (ddd, $J=7.8,6.8,1.2 \mathrm{~Hz}$, 1H) ppm. ${ }^{13} \mathrm{C}\left\{{ }^{1} \mathrm{H}\right\}$ NMR (101 MHz, $\left.\mathrm{CDCl}_{3}: \mathrm{CD}_{3} \mathrm{CN} ; 10: 1, \delta\right): 166.2$, 134.8, 131.8, 130.4, 125.4, 116.6, $115.4 \mathrm{ppm}$.

Synthesis of $t$ BuABX (1d). Following a reported procedure, ${ }^{27}$ iodine (11.42 g, $45.00 \mathrm{mmol}, 0.50$ equiv) was dissolved in TFA $(90 \mathrm{~mL}$; some iodine remained undissolved) to give a violet solution. Under stirring, this mixture was treated with aq. $\mathrm{HCl}(37 \% \mathrm{w} / \mathrm{w}$; $1.1 \mathrm{~mL}, 13 \mathrm{mmol}, 0.15$ equiv) and aq. $\mathrm{NaNO}_{2}(40 \% \mathrm{w} / \mathrm{w} ; 0.776 \mathrm{~g}$, $4.50 \mathrm{mmol}, 0.05$ equiv), which resulted in the rapid darkening of the solution to red-brown-black. (1.0 mmol). 4-tert-Butyltoluene (19) ( $15.5 \mathrm{~mL}, 90.0 \mathrm{mmol}, 1.0$ equiv) was finally added. Using a balloon, oxygen was bubbled through the mixture. Stirring was then continued under oxygen for $4 \mathrm{~h}$, after which the reaction was quenched by the addition of saturated sodium thiosulfate $(200 \mathrm{~mL})$ and sat. aq. $\mathrm{NaHCO}_{3}(200 \mathrm{~mL})$. The mixture was partitioned between dichloromethane $(400 \mathrm{~mL})$ and deionized water $(400 \mathrm{~mL})$. The organic layer was collected, dried over $\mathrm{MgSO}_{4}$, filtered, and concentrated under vacuum to provide a red-brown crude oil. The latter was purified by flash chromatography (silica; pentane) to give 4-(tert-butyl)-2-iodo-1methylbenzene (20) (24.6 g, $90.0 \mathrm{mmol}$, quantitative yield) as a bright yellow oil. ${ }^{1} \mathrm{H}$ NMR $\left(400 \mathrm{MHz}, \mathrm{CDCl}_{3}, \delta\right): 7.80(\mathrm{~d}, J=2.0 \mathrm{~Hz}$, $1 \mathrm{H}), 7.27(\mathrm{q}, J=2.4,1.9 \mathrm{~Hz}, 1 \mathrm{H}), 7.16(\mathrm{~d}, J=7.7 \mathrm{~Hz}, 1 \mathrm{H}), 2.39$ $(\mathrm{s}, 3 \mathrm{H}), 1.29(\mathrm{~s}, 9 \mathrm{H}) \mathrm{ppm}$. The signals of the ${ }^{1} \mathrm{H}$ NMR spectra were in accordance with the data reported in the literature. ${ }^{28}$

Following a slightly modified reported procedure, ${ }^{29} 4$-(tert-butyl)2-iodo-1-methylbenzene (20) (15.0 g, $54.7 \mathrm{mmol}, 1.0$ equiv) was dissolved in a mixture of water $(175 \mathrm{~mL})$ and pyridine $(220 \mathrm{~mL})$. Potassium permanganate $(34.6 \mathrm{~g}, 219 \mathrm{mmol}, 4.0$ equiv) was added to the mixture, followed by tetrabutylammonium iodide $(0.303 \mathrm{~g}$, $0.821 \mathrm{mmol}, 15 \mathrm{~mol} \%)$. The violet suspension was refluxed under vigorous stirring for 3 days. It was then allowed to cool down to room temperature, and the solids were filtered off through a plug of Celite, which was then washed with aq. $\mathrm{NaOH}(2.0 \mathrm{M} ; 300 \mathrm{~mL})$. Most of the pyridine was removed from the filtrate by evaporation under reduced pressure. The aqueous residue was washed with diethyl ether $(3 \times$ $200 \mathrm{~mL}$ ), and it was then acidified until $\mathrm{pH}<2$ by careful addition of aq. $\mathrm{HCl}(37 \% \mathrm{v} / \mathrm{v})$. The aqueous layer was extracted with DCM $(3 \times$ $200 \mathrm{~mL}$ ), and the combined organic extracts were dried over $\mathrm{MgSO}_{4}$, filtered, and concentrated under vacuum to furnish the desired product as a pale yellow solid. The latter was recrystallized from hexane and chloroform $(30 \mathrm{~mL}+8 \mathrm{~mL})$ to furnish highly pure 4-(tert-butyl)-2-iodobenzoic acid (21) (8.10 g, $26.6 \mathrm{mmol}, 48 \%)$ as a 
sticky, pale yellow crystalline solid. ${ }^{1} \mathrm{H}$ NMR $\left(400 \mathrm{MHz} \mathrm{CDCl}_{3}, \delta\right)$ : $11.81($ br s, $1 \mathrm{H}), 8.05(\mathrm{~d}, J=1.9 \mathrm{~Hz}, 1 \mathrm{H}), 7.99(\mathrm{~d}, J=8.3 \mathrm{~Hz}, 1 \mathrm{H})$, $7.46(\mathrm{dd}, J=8.3,2.0 \mathrm{~Hz}, 1 \mathrm{H}), 1.33(\mathrm{~s}, 9 \mathrm{H}) \mathrm{ppm} .{ }^{13} \mathrm{C}\left\{{ }^{1} \mathrm{H}\right\} \mathrm{NMR}$ $\left(101 \mathrm{MHz}, \mathrm{CDCl}_{3}, \delta\right): 171.4,157.6,139.3,132.0,130.0,125.2$, 95.2, $34.8,30.9 \mathrm{ppm}$. The signals of the ${ }^{1} \mathrm{H}$ NMR spectra were in accordance with the data reported in the literature. ${ }^{29}$

Caution: For safety reasons, the reaction was carried out behind an antiblast shield. Following a slightly modified reported procedure, ${ }^{29}$ 4-(tert-butyl)-2-iodobenzoic acid (21) $(7.70 \mathrm{~g}, 25.3 \mathrm{mmol}, 1.0$ equiv) and $\mathrm{NaIO}_{4}(5.42 \mathrm{~g}, 25.3 \mathrm{mmol}, 1.0$ equiv) were suspended in a 7:3 mixture of water $(34.4 \mathrm{~mL})$ and $\mathrm{AcOH}(14.7 \mathrm{~mL})$. The mixture was stirred at $110{ }^{\circ} \mathrm{C}$ for $4 \mathrm{~h}$ (behind a safety shield). Full dissolution of the solids was observed a few minutes after reaching the reflux temperature, to give a clear, pale yellow solution. Stirring was continued while protecting the mixture from light with aluminum foil. Ice-cold water $(35 \mathrm{~mL})$ was added under stirring, which resulted in the immediate precipitation of a colorless solid. The mixture was allowed to cool down to room temperature, while being protected from light with aluminum foil. It was then filtered, and the solid was washed with ice-cold water $(3 \times 30 \mathrm{~mL})$ and cold acetone $(3 \times 30 \mathrm{~mL})$. The resulting colorless solid $(\mathrm{HO}(t \mathrm{Bu}) \mathrm{BX}(7.15 \mathrm{~g}, 22.3 \mathrm{mmol}, 88 \%)$ was dried in the air overnight and then directly used in the next step.

Caution: For safety reasons, the reaction was carried out behind an antiblast shield. The solid obtained from the previous step (7.07 g, $22.1 \mathrm{mmol}$ ) was suspended in acetic anhydride $(20 \mathrm{~mL})$. The suspension was heated to reflux $\left(140{ }^{\circ} \mathrm{C}\right)$ while being stirred. The complete dissolution of the solid was observed when the temperature was ca. $125^{\circ} \mathrm{C}$ : at this point, the suspension converted into an offwhite, clear solution. After the temperature reached $140{ }^{\circ} \mathrm{C}$, stirring was continued for $2 \mathrm{~h}$. During this time, the solution became yellow. Heating was then stopped, and the solution was allowed to cool down to room temperature, with incipient precipitation of a colorless crystalline solid. The flask was then cooled to $-18{ }^{\circ} \mathrm{C}$ for $3 \mathrm{~h}$. $\mathrm{AcO}(t \mathrm{Bu}) \mathrm{BX}(\mathbf{2 2})(6.80 \mathrm{~g}, 18.8 \mathrm{mmol}, 85 \%$ yield; $75 \%$ over 2 steps, starting from 4-(tert-butyl)-2-iodobenzoic acid (21)) was then collected by filtration and washed with several portions of pentane. ${ }^{1} \mathrm{H}$ NMR $\left(400 \mathrm{MHz}, \mathrm{CDCl}_{3}, \delta\right): 8.15(\mathrm{~d}, J=8.0 \mathrm{~Hz}, 1 \mathrm{H}), 7.95(\mathrm{~d}, J=$ $1.5 \mathrm{~Hz}, 1 \mathrm{H}), 7.71(\mathrm{dd}, J=8.0,1.6 \mathrm{~Hz}, 1 \mathrm{H}), 2.27(\mathrm{~s}, 3 \mathrm{H}), 1.41(\mathrm{~s}, 9 \mathrm{H})$ ppm. ${ }^{13} \mathrm{C}\left\{{ }^{1} \mathrm{H}\right\}$ NMR $\left(101 \mathrm{MHz}, \mathrm{CDCl}_{3}, \delta\right): 176.2,168.3,161.0$, $132.8,128.9,126.2,125.6,119.0,36.2,31.1,20.3 \mathrm{ppm}$. The signals of the NMR spectra were in accordance with the data reported in the literature. ${ }^{29}$

Caution: For safety reasons, the reaction was carried out behind an antiblast shield. Following a slightly modified reported procedure, $\mathrm{AcO}(t \mathrm{Bu}) \mathrm{BX}(22)(4.09 \mathrm{~g}, 11.3 \mathrm{mmol}, 1.0$ equiv) was dissolved in DCM (dry; $11.3 \mathrm{~mL}$ ) to provide a clear, pale yellow solution. The latter was cooled to $0{ }^{\circ} \mathrm{C}$ (ice-water bath), and TMS-azide $(2.3 \mathrm{~mL}$, $16 \mathrm{mmol}, 1.4$ equiv) was added via syringe. Finally, TMS-triflate $(0.10 \mathrm{~mL}, 0.56 \mathrm{mmol}, 5 \mathrm{~mol} \%)$ was added at $0{ }^{\circ} \mathrm{C}$. Immediately, the reaction mixture turned bright yellow. After $2-3 \mathrm{~min}$, a pale yellow solid precipitated. Stirring was continued at $0{ }^{\circ} \mathrm{C}$ for $45 \mathrm{~min}$. The cooling bath was removed, and hexane $($ dry; $50 \mathrm{~mL})$ was added under stirring. The mixture was cooled to $-20{ }^{\circ} \mathrm{C}$ for $30 \mathrm{~min}$. Finally, the supernatant liquid was removed by suction filtration to provide $t \mathrm{BuABX}(1 \mathrm{~d})(3.41 \mathrm{~g}, 9.88 \mathrm{mmol}, 87 \%)$ as a pale yellow solid, which was washed with several portions of pentane and allowed to dry in the air. ${ }^{1} \mathrm{H}$ NMR $\left(400 \mathrm{MHz}, \mathrm{CDCl}_{3}, \delta\right): 8.20(\mathrm{~d}, J=8.0 \mathrm{~Hz}, 1 \mathrm{H}), 7.93$ $(\mathrm{d}, J=1.5 \mathrm{~Hz}, 1 \mathrm{H}), 7.77(\mathrm{dd}, J=8.0,1.5 \mathrm{~Hz}, 1 \mathrm{H}), 1.44(\mathrm{~s}, 9 \mathrm{H}) \mathrm{ppm}$. ${ }^{13} \mathrm{C}\left\{{ }^{1} \mathrm{H}\right\}$ NMR $\left(101 \mathrm{MHz}, \mathrm{CDCl}_{3}, \delta\right): 167.3,161.4,133.1,129.3$, $126.7,122.9,118.3,36.3,31.1 \mathrm{ppm}$. HRMS (ESI):: $[\mathrm{M}+\mathrm{H}]^{+}$calcd. for $\mathrm{C}_{11} \mathrm{H}_{13} \mathrm{IN}_{3} \mathrm{O}_{2}{ }^{+}, 346.0047$; found, 346.0042. IR (film): 2968 (w), $2056(\mathrm{~s}), 1661(\mathrm{~m}), 1651(\mathrm{~m}), 1595(\mathrm{w}), 1549(\mathrm{w}), 1390(\mathrm{w})$, $1280(\mathrm{~m}), 1232(\mathrm{~m}), 1156(\mathrm{w}), 1137(\mathrm{w}), 859(\mathrm{w}) \mathrm{cm}^{-1}$.

Synthesis of ABZ (2). $\mathrm{SOCl}_{2}(3.53 \mathrm{~mL}, 48.4 \mathrm{mmol}, 1.2$ equiv) followed by DMF $(0.20 \mathrm{~mL}, 2.6 \mathrm{mmol}, 0.06$ equiv) were added dropwise to a solution of 2-iodobenzoic acid (18) (10.0 g, $40.3 \mathrm{mmol}$, 1 equiv) in $\mathrm{CH}_{2} \mathrm{Cl}_{2}(200 \mathrm{~mL}, 0.2 \mathrm{~m})$, and the resulting mixture was stirred for $3 \mathrm{~h}$ at room temperature. Thereafter, the solvent was evaporated, the residue was dissolved in toluene $(32 \mathrm{~mL})$, and then the mixture was added to a solution of 4-toluenesulfoneamide $(6.90 \mathrm{~g}$,
$40.3 \mathrm{mmol}, 1.00$ equiv), triethylamine ( $14.0 \mathrm{~mL}, 101 \mathrm{mmol}, 2.50$ equiv), and DMAP (0.250 g, $2.02 \mathrm{mmol}, 0.05$ equiv) in EtOAc $(80 \mathrm{~mL})$. The mixture was heated for $1 \mathrm{~h}$ to $60^{\circ} \mathrm{C}$, then cooled to room temperature, and quenched with $1 \mathrm{~m} \mathrm{HCl}(50 \mathrm{~mL})$. The mixture was extracted with EtOAc $(3 \times 50 \mathrm{~mL})$, and the combined organic extracts were washed with brine and dried over $\mathrm{MgSO}_{4}$. The drying agent was filtered off, and the solvent was evaporated. The residue was purified by column chromatography (silica, $\mathrm{CH}_{2} \mathrm{Cl}_{2}$ ) affording 2-iodo-N-tosylbenzamide (23) $(15.8 \mathrm{~g}, 39.5 \mathrm{mmol}, 98 \%)$ as a colorless oil. $R_{f}$ (silica, $\left.\mathrm{CH}_{2} \mathrm{Cl}_{2}\right)$ : 0.5. ${ }^{1} \mathrm{H}$ NMR $\left(400 \mathrm{MHz}, \mathrm{CDCl}_{3}, \delta\right): 8.78(\mathrm{~s}, 1 \mathrm{H}), 8.01(\mathrm{~d}, J=8.4 \mathrm{~Hz}$, $2 \mathrm{H}), 7.80(\mathrm{dd}, J=8.0,1.0 \mathrm{~Hz}, 1 \mathrm{H}), 7.43-7.31(\mathrm{~m}, 4 \mathrm{H}), 7.10$ (ddd, $J=8.0,7.2,2.0 \mathrm{~Hz}, 1 \mathrm{H}), 2.44(\mathrm{~s}, 3 \mathrm{H}) \mathrm{ppm} .{ }^{13} \mathrm{C}\left\{{ }^{1} \mathrm{H}\right\} \mathrm{NMR}(101 \mathrm{MHz}$, $\left.\mathrm{CDCl}_{3}, \delta\right): 165.6,145.3,140.2,138.4,134.9,132.4,129.5,128.8,128.7$, 128.3, 91.6, 21.7 ppm. HRMS (ESI): $[\mathrm{M}+\mathrm{H}]^{+}$calcd. for $\mathrm{C}_{14} \mathrm{H}_{13} \mathrm{INO}_{3} \mathrm{~S}^{+}, 401.9655$; found, 401.9653. IR (film): $3222(\mathrm{w})$, $2257(\mathrm{w}), 1702(\mathrm{~m}), 1598(\mathrm{w}), 1431(\mathrm{~m}), 1346(\mathrm{~m}), 1237(\mathrm{w}), 1167$ (s), $1083(\mathrm{~s}), 1016(\mathrm{~m}), 911(\mathrm{~m}), 888(\mathrm{~m}), 834(\mathrm{~m}) \mathrm{cm}^{-1}$.

Caution: For safety reasons, the reaction was carried out behind an antiblast shield. mCPBA (77\% pure; $8.85 \mathrm{~g}, 39.6 \mathrm{mmol}, 1.00$ equiv) was added to a solution of 23 ( $15.8 \mathrm{~g}, 39.5 \mathrm{mmol}, 1.00$ equiv) and $\mathrm{Ac}_{2} \mathrm{O}(160 \mathrm{~mL}, 1.68 \mathrm{~mol}, 42.4$ equiv) in $\mathrm{AcOH}(158 \mathrm{~mL}, 0.10 \mathrm{M})$, and the resulting mixture was heated for $48 \mathrm{~h}$ to $80{ }^{\circ} \mathrm{C}$. Thereafter, the mixture was cooled to room temperature, and diethyl ether $(150 \mathrm{~mL})$ was added. Compound 24 (7.13 g, $15.5 \mathrm{mmol}, 39 \%)$ crystallized at $0{ }^{\circ} \mathrm{C}$ from the solution and was collected by filtration. $\mathrm{mp}$ $161{ }^{\circ} \mathrm{C}$ (decomposition). ${ }^{1} \mathrm{H}$ NMR (400 MHz, $d_{6}$-DMSO, $\delta$ ): 8.02$7.95(\mathrm{~m}, 2 \mathrm{H}), 7.95-7.89(\mathrm{~m}, 2 \mathrm{H}), 7.86(\mathrm{dd}, J=8.8,0.9 \mathrm{~Hz}, 1 \mathrm{H})$, $7.80-7.71(\mathrm{~m}, 1 \mathrm{H}), 7.44(\mathrm{~d}, J=8.1 \mathrm{~Hz}, 2 \mathrm{H}), 2.38(\mathrm{~s}, 3 \mathrm{H}), 2.26$ (s, 3H) ppm. ${ }^{13} \mathrm{C}\left\{{ }^{1} \mathrm{H}\right\}$ NMR (101 MHz, $d_{6}$-DMSO, $\delta$ ): 174.1, 162.4, $144.5,136.8,136.0,132.3,131.4,131.2$, 129.7, 128.8, 127.9, 118.0, $21.1,20.2$ ppm. HRMS (ESI): $[\mathrm{M}+\mathrm{Na}]^{+}$calcd. for $\mathrm{C}_{16} \mathrm{H}_{14} \mathrm{INNaO}_{5} \mathrm{~S}^{+}$, 481.9530; found, 481.9537. IR (film): $3062(\mathrm{w})$, $1673(\mathrm{~m}), 1585$ (w), 1440 (w), $1334(\mathrm{~m}), 1267$ (s), 1149 (s), 1085 (m), $1009(\mathrm{w}), 908(\mathrm{~m}), 845(\mathrm{~s}) \mathrm{cm}^{-1}$.

Caution: For safety reasons, the reaction was carried out behind an antiblast shield. TMSOTf ( $14 \mu \mathrm{L}, 0.080 \mathrm{mmol}, 0.005$ equiv) was added dropwise to a suspension of $24(7.13 \mathrm{~g}, 15.5 \mathrm{mmol}, 1$ equiv) and $\mathrm{TMSN}_{3}\left(3.0 \mathrm{~mL}, 23 \mathrm{mmol}, 1.5\right.$ equiv) in $\mathrm{CH}_{2} \mathrm{Cl}_{2}(31 \mathrm{~mL})$ at $0{ }^{\circ} \mathrm{C}$. The resulting mixture was stirred for $30 \mathrm{~min}$ at $0{ }^{\circ} \mathrm{C}$, and thereafter, the formed solid was filtered, washed with $\mathrm{CH}_{2} \mathrm{Cl}_{2}$ followed by pentane, and dried in vacuo, affording $\mathrm{ABZ}$ (2) (5.38 g, $12.2 \mathrm{mmol}$, $78 \%)$ as a pale yellow solid. $\mathrm{mp} 166{ }^{\circ} \mathrm{C}$ (decomposition). ${ }^{1} \mathrm{H}$ NMR $\left(400 \mathrm{MHz}, d_{6}\right.$-DMSO, $\delta$ ): $8.17(\mathrm{dd}, J=8.3,0.9 \mathrm{~Hz}, 1 \mathrm{H}), 8.03-7.93$ $(\mathrm{m}, 2 \mathrm{H}), 7.93-7.87(\mathrm{~m}, 2 \mathrm{H}), 7.75(\mathrm{td}, J=7.4,0.9 \mathrm{~Hz}, 1 \mathrm{H}), 7.46-$ $7.37(\mathrm{~m}, 2 \mathrm{H}), 2.38(\mathrm{~s}, 3 \mathrm{H}) \mathrm{ppm} .{ }^{13} \mathrm{C}\left\{{ }^{1} \mathrm{H}\right\}$ NMR (101 MHz, $d_{6}$-DMSO, $\delta$ ): 162.6, 144.0, 136.7, 136.3, 132.5, 131.2, 131.2, 129.5, 128.5, 127.8, 118.2, $21.1 \mathrm{ppm}$. HRMS (ESI): $[\mathrm{M}+\mathrm{Na}]^{+}$calcd for $\mathrm{C}_{14} \mathrm{H}_{11} \mathrm{IN}_{4} \mathrm{NaO}_{3} \mathrm{~S}^{+}$, 464.9489; found, 464.9487. IR (film): $2052(\mathrm{~m})$, $1671(\mathrm{~m}), 1437$ (w), $1286(\mathrm{~s}), 1266(\mathrm{~m}), 1212(\mathrm{~m}), 1147(\mathrm{~s}), 1084$ (m), $900(\mathrm{~s}), 849(\mathrm{~m}) \mathrm{cm}^{-1}$.

Synthesis of 1-(1-Bromovinyl)-arenes. (1-Bromovinyl)benzene (25a) was commercially available and used as such without further purifications. All of the other compounds were synthesized as described below.

General Method A. Bromine ( 1.25 equiv) was added to a cold solution of triphenyl phosphite (1.1 equiv) in $\mathrm{CH}_{2} \mathrm{Cl}_{2}(0.3 \mathrm{M})$ at $-60{ }^{\circ} \mathrm{C}$. Then, a solution of triethylamine (1.35 equiv) and the acetophenone (1.0 equiv) in $\mathrm{CH}_{2} \mathrm{Cl}_{2}(1 \mathrm{M})$ was added dropwise to the pale orange solution, and the reaction mixture was thereafter stirred for $18 \mathrm{~h}$, while warming to room temperature. Then, the mixture was heated to reflux for another $2 \mathrm{~h}$. The reaction was quenched with sat. aq. $\mathrm{Na}_{2} \mathrm{~S}_{2} \mathrm{O}_{3}(50 \mathrm{~mL})$ and extracted with $\mathrm{CH}_{2} \mathrm{Cl}_{2}(3 \times 30 \mathrm{~mL})$. The combined organic extracts were washed with brine $(150 \mathrm{~mL})$ and dried over sodium sulfate, the drying agent was filtered off, and the solvent was evaporated. The residue was purified by column chromatography (silica, pentane:EtOAc 1:0 to 95:5) affording the desired 1-(1-bromovinyl)-arene 25, which, due to low stablility, was used immediately in the next step.

General Method B. Bromine (1.2 equiv) was added dropwise to a solution of the styrene (1.0 equiv) in $\mathrm{CH}_{2} \mathrm{Cl}_{2}(20 \mathrm{~mL})$ over $5 \mathrm{~min}$ 
at $0{ }^{\circ} \mathrm{C}$. Thereafter, the reaction mixture was stirred for $15 \mathrm{~min}$ at $0{ }^{\circ} \mathrm{C}$ and then quenched with sat. aq. $\mathrm{Na}_{2} \mathrm{~S}_{2} \mathrm{O}_{3}$. The mixture was extracted with $\mathrm{CH}_{2} \mathrm{Cl}_{2}(3 \times 30 \mathrm{~mL})$, and the combined organic extracts were washed with brine and dried over $\mathrm{Na}_{2} \mathrm{SO}_{4}$. The drying agent was filtered off, and the solvent was evaporated. The residue was dissolved in THF $/ \mathrm{CH}_{3} \mathrm{OH}$ 1:1 $(0.5 \mathrm{~m}), \mathrm{K}_{2} \mathrm{CO}_{3}$ (2.0 equiv) was added, and the mixture was stirred for $3 \mathrm{~h}$ at room temperature. Thereafter, the solvent was evaporated, and the residue was suspended in water $(50 \mathrm{~mL})$ and then extracted with pentane $(3 \times 50 \mathrm{~mL})$. The combined organic extracts were washed with brine $(150 \mathrm{~mL})$ and dried over $\mathrm{Na}_{2} \mathrm{SO}_{4}$. The drying agent was filtered off, and the solvent was evaporated. The residue was purified by column chromatography (silica, pentane), affording the desired 1-(1-bromovinyl)-arene 25 , which, due to low stablility, was used immediately in the next step.

1-(1-Bromovinyl)-4-methylbenzene (25b).

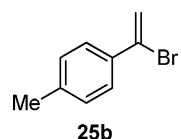

Compound $\mathbf{2 5 b}$ was prepared according to the general method A, starting with 1 -( $p$-tolyl)ethanone $(1.1 \mathrm{~mL}, 9.3 \mathrm{mmol}, 1.0$ equiv), $\mathrm{Br}_{2}$ (0.60 mL, $12 \mathrm{mmol}, 1.2$ equiv), triphenyl phosphite $(2.7 \mathrm{~mL}$, $10 \mathrm{mmol}, 1.1$ equiv), and $\mathrm{Et}_{3} \mathrm{~N}$ (1.7 mL, 13 mmol, 1.35 equiv). The title compound $\mathbf{2 5} \mathbf{b}$ was obtained as a colorless liquid $(1.2 \mathrm{~g}$, $6.1 \mathrm{mmol}, 65 \%$ yield). ${ }^{1} \mathrm{H} \mathrm{NMR}\left(400 \mathrm{MHz}, \mathrm{CDCl}_{3}, \delta\right.$ ): $7.60-$ $7.44(\mathrm{~m}, 2 \mathrm{H}), 7.24-7.07(\mathrm{~m}, 2 \mathrm{H}), 6.10(\mathrm{~s}, 1 \mathrm{H}), 5.75\left(\mathrm{~s}, 1 \mathrm{H}_{2}\right)$, $2.38(\mathrm{~s}, 3 \mathrm{H}) \mathrm{ppm}$. The signals of the ${ }^{1} \mathrm{H}$ NMR spectra were in accordance with the data reported in the literature. ${ }^{30}$

1-(1-Bromovinyl)-4-(tert-butyl)benzene (25c).

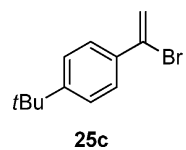

Compound 25c was prepared according to the general method B, starting with 1-(tert-butyl)-4-vinylbenzene $(1.9 \mathrm{~mL}, 10 \mathrm{mmol}$, 1.0 equiv), $\mathrm{Br}_{2}$ (0.60 mL, $12 \mathrm{mmol}, 1.2$ equiv), and $\mathrm{K}_{2} \mathrm{CO}_{3}$ (2.8 g, $20 \mathrm{mmol}, 2.0$ equiv). The title compound $25 \mathrm{c}$ was obtained as a colorless liquid ( $2.0 \mathrm{~g}, 8.4 \mathrm{mmol}, 84 \%$ yield). ${ }^{1} \mathrm{H}$ NMR (400 MHz, $\mathrm{CDCl}_{3}, \delta$ ): 7.58-7.49 (m, 2H), 7.42$7.34(\mathrm{~m}, 2 \mathrm{H}), 6.10(\mathrm{~d}, J=2.0 \mathrm{~Hz}, 1 \mathrm{H}), 5.74(\mathrm{~d}, J=2.0 \mathrm{~Hz}$, $1 \mathrm{H}), 1.33(\mathrm{~s}, 9 \mathrm{H}) \mathrm{ppm}$. The signals of the ${ }^{1} \mathrm{H}$ NMR spectra were in accordance with the data reported in the literature. ${ }^{31}$

4-(1-Bromovinyl)-1,1'-biphenyl (25d).

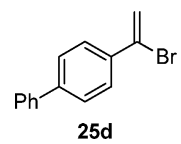

Compound 25d was prepared according to the general method A, starting with 1-([1,1'-biphenyl]-4-yl)ethanone $(2.1 \mathrm{~g}, 11 \mathrm{mmol}$, 1.0 equiv), $\mathrm{Br}_{2}$ ( $0.62 \mathrm{~mL}, 12 \mathrm{mmol}, 1.2$ equiv), triphenyl phosphite (3.1 mL, $12 \mathrm{mmol}, 1.10$ equiv), and $\mathrm{Et}_{3} \mathrm{~N}(2.0 \mathrm{~mL}, 14 \mathrm{mmol}$, 1.35 equiv). The title compound $25 \mathbf{d}$ was obtained as a colorless oil (1.6 g, $6.2 \mathrm{mmol}, 58 \%$ yield). ${ }^{1} \mathrm{H}$ NMR (400 $\left.\mathrm{MHz}, \mathrm{CDCl}_{3}, \delta\right)$ : 7.70-7.65 (m, 2H), 7.63-7.56 (m, 4H), 7.50-7.42 (m, 2H), $7.40-7.33(\mathrm{~m}, 1 \mathrm{H}), 6.18(\mathrm{~d}, J=2.0 \mathrm{~Hz}, 1 \mathrm{H}), 5.81(\mathrm{~d}, J=2.0 \mathrm{~Hz}$, $1 \mathrm{H}) \mathrm{ppm}$. The signals of the ${ }^{1} \mathrm{H}$ NMR spectra were in accordance with the data reported in the literature. ${ }^{32}$

1-(1-Bromovinyl)-4-methoxybenzene (25e).

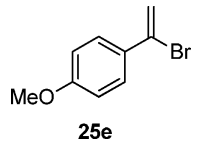

Compound 25e was prepared according to general method A, starting with 1-(4-methoxyphenyl)ethanone (1.4 g, $9.3 \mathrm{mmol}, 1.0$ equiv), $\mathrm{Br}_{2}$ (0.62 mL, $12 \mathrm{mmol}, 1.25$ equiv), triphenyl phosphite $(2.7 \mathrm{~mL}$, $10 \mathrm{mmol}, 1.1$ equiv), and $\mathrm{Et}_{3} \mathrm{~N}$ (1.8 mL, $13 \mathrm{mmol}, 1.35$ equiv). The title compound 25e was obtained as a colorless oil $(1.5 \mathrm{~g}, 7.0 \mathrm{mmol}$, $76 \%$ yield). ${ }^{1} \mathrm{H}$ NMR $\left(400 \mathrm{MHz}, \mathrm{CDCl}_{3}, \delta\right): 7.64-7.51(\mathrm{~m}, 2 \mathrm{H})$, $6.90-6.76(\mathrm{~m}, 2 \mathrm{H}), 6.02(\mathrm{~d}, J=2.0 \mathrm{~Hz}, 1 \mathrm{H}), 5.68(\mathrm{~d}, J=2.0 \mathrm{~Hz}$, $1 \mathrm{H}), 3.83(\mathrm{~s}, 3 \mathrm{H}) \mathrm{ppm}$. The signals of the ${ }^{1} \mathrm{H}$ NMR spectra were in accordance with the data reported in the literature. ${ }^{33}$

1-(1-Bromovinyl)-4-fluorobenzene (25f).<smiles>C=C(Br)c1ccc(F)cc1</smiles>

Compound 25f was prepared according to general method B, starting with 1-fluoro-4-vinylbenzene $(1.2 \mathrm{~mL}, 10 \mathrm{mmol}$, 1.0 equiv), $\mathrm{Br}_{2}$ ( $0.62 \mathrm{~mL}, 12 \mathrm{mmol}, 1.2$ equiv), and $\mathrm{K}_{2} \mathrm{CO}_{3}(2.8 \mathrm{~g}$, $20 \mathrm{mmol}, 2.0$ equiv). The title compound $\mathbf{2 5 f}$ was obtained as a colorless liquid (1.6 g, $8.0 \mathrm{mmol}, 80 \%$ yield). ${ }^{1} \mathrm{H}$ NMR $\left(400 \mathrm{MHz}, \mathrm{CDCl}_{3}, \delta\right): 7.61-7.53(\mathrm{~m}, 2 \mathrm{H}), 7.12-6.97(\mathrm{~m}, 2 \mathrm{H})$, $6.06(\mathrm{~d}, J=2.1 \mathrm{~Hz}, 1 \mathrm{H}), 5.76(\mathrm{~d}, J=2.1 \mathrm{~Hz}, 1 \mathrm{H}) \mathrm{ppm}$. The signals of the ${ }^{1} \mathrm{H}$ NMR spectra were in accordance with the data reported in the literature. ${ }^{34}$

1-(1-Bromovinyl)-4-chlorobenzene (25g).<smiles>C=C(Br)c1ccc(Cl)cc1</smiles>

Compound 25g was prepared according to general method B, starting with 1-chloro-4-vinylbenzene $(1.3 \mathrm{~mL}, 10 \mathrm{mmol}$, 1.0 equiv), $\mathrm{Br}_{2}$ ( $0.62 \mathrm{~mL}, 12 \mathrm{mmol}, 1.2$ equiv), and $\mathrm{K}_{2} \mathrm{CO}_{3}(2.8 \mathrm{~g}$, $20 \mathrm{mmol}, 2.0$ equiv). The title compound $25 \mathrm{~g}$ was obtained as a colorless liquid (1.0 g, $4.6 \mathrm{mmol}, 46 \%$ yield). ${ }^{1} \mathrm{H}$ NMR $\left(400 \mathrm{MHz}, \mathrm{CDCl}_{3}, \delta\right): 7.59-7.48(\mathrm{~m}, 2 \mathrm{H}), 7.36-7.29(\mathrm{~m}, 2 \mathrm{H})$, $6.11(\mathrm{~d}, J=2.2 \mathrm{~Hz}, 1 \mathrm{H}), 5.79(\mathrm{~d}, J=2.2 \mathrm{~Hz}, 1 \mathrm{H}) \mathrm{ppm}$. The signals of the ${ }^{1} \mathrm{H}$ NMR spectra were in accordance with the data reported in the literature. ${ }^{34}$

1-(1-Bromovinyl)-3-methylbenzene (25h).<smiles>C=C(Br)c1cccc(C)c1</smiles>

$25 \mathrm{~h}$

Compound 25h was prepared according to general method B, starting with 1-methyl-3-vinylbenzene $(1.3 \mathrm{~mL}, 10 \mathrm{mmol}$, 1.0 equiv), $\mathrm{Br}_{2}$ (0.62 mL, $12 \mathrm{mmol}, 1.2$ equiv), and $\mathrm{K}_{2} \mathrm{CO}_{3}$ ( $2.8 \mathrm{~g}, 20 \mathrm{mmol}, 2.0$ equiv). The title compound $25 \mathrm{~h}$ was obtained as a colorless liquid ( $1.0 \mathrm{~g}, 5.1 \mathrm{mmol}, 51 \%$ yield). ${ }^{1} \mathrm{H}$ NMR (400 MHz, $\mathrm{CDCl}_{3}, \delta$ ): 7.42-7.38 (m, 2H), 7.27$7.20(\mathrm{~m}, 1 \mathrm{H}), 7.18-7.14(\mathrm{~m}, 1 \mathrm{H}), 6.11(\mathrm{~d}, J=2.0 \mathrm{~Hz}, 1 \mathrm{H})$, $5.77(\mathrm{~d}, J=2.0 \mathrm{~Hz}, 1 \mathrm{H}), 2.38(\mathrm{~s}, 3 \mathrm{H}) \mathrm{ppm}$. The signals of the ${ }^{1} \mathrm{H}$ NMR spectra were in accordance with the data reported in the literature. $^{30}$

1-(1-Bromovinyl)-3-methoxybenzene (25i).<smiles>C=C(Br)c1cccc(OC)c1</smiles>

Compound 25i was prepared according to general method A, starting with 1-(3-methoxyphenyl)ethanone (1.3 mL, $9.3 \mathrm{mmol}$, 1.0 equiv), $\mathrm{Br}_{2}(0.62 \mathrm{~mL}, 12 \mathrm{mmol}, 1.25$ equiv), triphenyl phosphite $\left(2.7 \mathrm{~mL}, 10 \mathrm{mmol}, 1.1\right.$ equiv), and $\mathrm{Et}_{3} \mathrm{~N}$ (1.8 mL, 
13 mmol, 1.35 equiv). The title compound $25 \mathbf{i}$ was obtained as a colorless oil ( $0.50 \mathrm{~g}, 2.3 \mathrm{mmol}, 25 \%$ yield). ${ }^{1} \mathrm{H} \mathrm{NMR}(400 \mathrm{MHz}$, $\left.\mathrm{CDCl}_{3}, \delta\right): 7.32-7.26(\mathrm{~m}, 1 \mathrm{H}), 7.20(\mathrm{~m}, 1 \mathrm{H}), 7.16(\mathrm{~m}, 1 \mathrm{H})$, 6.95-6.89 (m, 1H), $6.15(\mathrm{~d}, J=2.0 \mathrm{~Hz}, 1 \mathrm{H}), 5.80(\mathrm{~d}, J=2.0 \mathrm{~Hz}$, $1 \mathrm{H}), 3.86(\mathrm{~s}, 3 \mathrm{H}) \mathrm{ppm}$. The signals of the ${ }^{1} \mathrm{H}$ NMR spectra were in accordance with the data reported in the literature. ${ }^{35}$

1-(1-Bromovinyl)-3-fluorobenzene (25j).

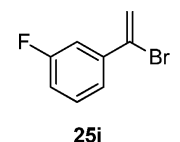

Compound $25 \mathbf{j}$ was prepared according to general method B, starting with 1-fluoro-3-vinylbenzene (1.2 mL, $10 \mathrm{mmol}$, 1.0 equiv), $\mathrm{Br}_{2}$ ( $0.62 \mathrm{~mL}, 12 \mathrm{mmol}, 1.2$ equiv), and $\mathrm{K}_{2} \mathrm{CO}_{3}(2.8 \mathrm{~g}$, $20 \mathrm{mmol}, 2.0$ equiv). The title compound $25 \mathbf{j}$ was obtained as a colorless liquid (1.6 g, $7.9 \mathrm{mmol}, 79 \%$ yield). ${ }^{1} \mathrm{H}$ NMR $\left(400 \mathrm{MHz}, \mathrm{CDCl}_{3}, \delta\right): 7.42-7.04(\mathrm{~m}, 3 \mathrm{H}), 7.03$ (m, 1H), 6.15 (d, $J=2.1 \mathrm{~Hz}, 1 \mathrm{H}), 5.82(\mathrm{~d}, J=2.2 \mathrm{~Hz}, 1 \mathrm{H}) \mathrm{ppm}$. The signals of the ${ }^{1} \mathrm{H}$ NMR spectra were in accordance with the data reported in the literature. ${ }^{30}$

1-(1-Bromovinyl)-2-methylbenzene (25k).

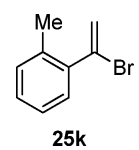

Compound 25k was prepared according to general method A, starting with 1-(o-tolyl)ethanone $(1.3 \mathrm{~mL}, 10 \mathrm{mmol}, 1.0$ equiv), $\mathrm{Br}_{2}(64 \mu \mathrm{L}, 13 \mathrm{mmol}, 1.25$ equiv), triphenyl phosphite $(2.9 \mathrm{~mL}$, $11 \mathrm{mmol}, 1.1$ equiv), and $\mathrm{Et}_{3} \mathrm{~N}$ (1.9 mL, 13 mmol, 1.35 equiv). The title compound $25 \mathrm{k}$ was obtained as a colorless oil (1.2 g, $6.2 \mathrm{mmol}, 62 \%$ yield). ${ }^{1} \mathrm{H} \mathrm{NMR}\left(400 \mathrm{MHz}, \mathrm{CDCl}_{3}, \delta\right): 7.34-$ $7.16(\mathrm{~m}, 4 \mathrm{H}), 5.92(\mathrm{~d}, J=1.5 \mathrm{~Hz}, 1 \mathrm{H}), 5.77(\mathrm{~d}, J=1.6 \mathrm{~Hz}, 1 \mathrm{H})$, $2.44(\mathrm{~s}, 3 \mathrm{H}) \mathrm{ppm}$. The signals of the ${ }^{1} \mathrm{H}$ NMR spectra were in accordance with the data reported in the literature. ${ }^{36}$

4-(1-Bromovinyl)-1,2-dimethylbenzene (25I).<smiles>C=C(Br)c1ccc(C)c(N)c1</smiles>

Compound 251 was prepared according to general method A, starting with 1-(3,4-dimethylphenyl)ethanone $(1.5 \mathrm{~mL}, 10 \mathrm{mmol}$, 1.0 equiv), $\mathrm{Br}_{2}$ (64 $\mu \mathrm{L}, 13 \mathrm{mmol}, 1.25$ equiv), triphenyl phosphite (2.9 mL, $11 \mathrm{mmol}, 1.1$ equiv), and $\mathrm{Et}_{3} \mathrm{~N}$ (1.9 mL, $14 \mathrm{mmol}$, 1.35 equiv). The title compound $\mathbf{2 5 1}$ was obtained as a colorless oil (1.3 g, $6.0 \mathrm{mmol}, 60 \%$ yield). ${ }^{1} \mathrm{H} \mathrm{NMR}\left(400 \mathrm{MHz} \mathrm{CDCl}_{3}, \delta\right)$ : 7.39 (s, 1H), 7.35 (d, $J=8.0 \mathrm{~Hz}, 1 \mathrm{H}), 7.13(\mathrm{~d}, J=8.0 \mathrm{~Hz}, 1 \mathrm{H})$, 6.16-6.03 (m, 1H), 5.81-5.69 (m, 1H), $2.31(\mathrm{~s}, 3 \mathrm{H}), 2.30$ (s, 3H) ppm. ${ }^{13} \mathrm{C}\left\{{ }^{1} \mathrm{H}\right\}$ NMR (101 MHz, $\left.\mathrm{CDCl}_{3}, \delta\right): 137.9,136.4$, 136.1, 131.2, 129.5, 128.4, 124.7, 116.6, 19.8, 19.5 ppm. HRMS (ESI): $\left[\mathrm{M}^{+}\right]$calcd. for $\mathrm{C}_{10} \mathrm{H}_{11} \mathrm{Br}, 211.0117$; found, 211.0117. IR (film): 2970 (w), 2921 (w), 1681 (w), 1605 (m), 1499 (w), $1449(\mathrm{w}), 1384(\mathrm{w}), 1241(\mathrm{~m}), 1215(\mathrm{w}), 1126(\mathrm{w}), 1065(\mathrm{~m})$, 880 (s), 821 (s), $729(\mathrm{~m}) \mathrm{cm}^{-1}$.

\section{4-(1-Bromovinyl)-1,2-dimethoxybenzene (25m).}

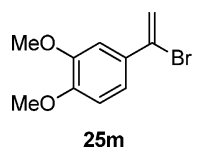

Compound $\mathbf{2 5 \mathrm { m }}$ was prepared according to general method $\mathrm{A}$, starting with 1-(3,4-dimethoxyphenyl)ethanone (1.7 g, $9.3 \mathrm{mmol}$, 1.0 equiv), $\mathrm{Br}_{2}(0.62 \mathrm{~mL}, 12 \mathrm{mmol}, 1.25$ equiv $)$, triphenyl phosphite $\left(2.7 \mathrm{~mL}, 10 \mathrm{mmol}, 1.1\right.$ equiv), and $\mathrm{Et}_{3} \mathrm{~N}$ (1.8 mL, $13 \mathrm{mmol}, 1.35$ equiv). The title compound $25 \mathrm{~m}$ was obtained as a colorless oil $(0.60 \mathrm{~g}, 2.5 \mathrm{mmol}, 27 \%$ yield $).) .{ }^{1} \mathrm{H}$ NMR $\left(400 \mathrm{MHz}, \mathrm{CDCl}_{3}, \delta\right.$ ): $7.17(\mathrm{dd}, J=8.4,2.3 \mathrm{~Hz}, 1 \mathrm{H}), 7.10$ $(\mathrm{d}, J=2.2 \mathrm{~Hz}, 1 \mathrm{H}), 6.82(\mathrm{~d}, J=8.4 \mathrm{~Hz}, 1 \mathrm{H}), 6.02(\mathrm{~d}, J=2.0 \mathrm{~Hz}$, $1 \mathrm{H}), 5.69(\mathrm{~d}, J=2.0 \mathrm{~Hz}, 1 \mathrm{H}), 3.91(\mathrm{~s}, 3 \mathrm{H}), 3.89(\mathrm{~s}, 3 \mathrm{H}) \mathrm{ppm}$. The signals of the ${ }^{1} \mathrm{H}$ NMR spectra were in accordance with the data reported in the literature. ${ }^{37}$

5-(1-Bromovinyl)benzo[d][1,3]dioxole (25n).

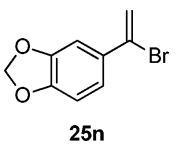

Compound 25n was prepared according to general method A, starting with 1-(benzo[d][1,3] dioxol-5-yl)ethanone (1.5 g, $9.3 \mathrm{mmol}, 1.0$ equiv), $\mathrm{Br}_{2}(0.62 \mathrm{~mL}, 12 \mathrm{mmol}, 1.25$ equiv), triphenyl phosphite $\left(2.7 \mathrm{~mL}, 10 \mathrm{mmol}, 1.1\right.$ equiv), and $\mathrm{Et}_{3} \mathrm{~N}$ (1.8 mL, 13 mmol, 1.35 equiv). The title compound $25 \mathrm{n}$ was obtained as a colorless oil (1.5 g, $6.6 \mathrm{mmol}, 71 \%$ yield). ${ }^{1} \mathrm{H}$ NMR $\left(400 \mathrm{MHz}, \mathrm{CDCl}_{3}, \delta\right): 7.11(\mathrm{dd}, J=8.1,1.9 \mathrm{~Hz}, 1 \mathrm{H}), 7.07$ $(\mathrm{d}, J=1.9 \mathrm{~Hz}, 1 \mathrm{H}), 6.77(\mathrm{~d}, J=8.1 \mathrm{~Hz}, 1 \mathrm{H}), 5.99(\mathrm{~m}, 3 \mathrm{H}), 5.67$ $(\mathrm{d}, J=2.0 \mathrm{~Hz}, 1 \mathrm{H}) \mathrm{ppm}$. The signals of the ${ }^{1} \mathrm{H}$ NMR spectra were in accordance with the data reported in the literature. ${ }^{38}$

2-(1-Bromovinyl)naphthalene (250).

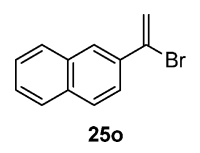

Compound 25o was prepared according to the general method $\mathrm{A}$, starting with 1-(naphthalen-2-yl)ethanone $(1.6 \mathrm{~g}, 9.3 \mathrm{mmol}$, 1.0 equiv), $\mathrm{Br}_{2}$ ( $0.62 \mathrm{~mL}, 12 \mathrm{mmol}, 1.25$ equiv), triphenyl phosphite $\left(2.7 \mathrm{~mL}, 10 \mathrm{mmol}, 1.1\right.$ equiv), and $\mathrm{Et}_{3} \mathrm{~N}$ (1.8 mL, $13 \mathrm{mmol}$, 1.35 equiv). The title compound 250 was obtained as a colorless oil (1.0 g, $4.3 \mathrm{mmol}, 46 \%$ yield). ${ }^{1} \mathrm{H} \mathrm{NMR}\left(400 \mathrm{MHz}, \mathrm{CDCl}_{3}, \delta\right)$ : $8.09(\mathrm{~d}, J=1.8 \mathrm{~Hz}, 1 \mathrm{H}), 7.90-7.78(\mathrm{~m}, 3 \mathrm{H}), 7.69$ (dd, $J=8.7$, $1.9 \mathrm{~Hz}, 1 \mathrm{H}), 7.56-7.43(\mathrm{~m}, 2 \mathrm{H}), 6.27(\mathrm{~d}, J=2.1 \mathrm{~Hz}, 1 \mathrm{H}), 5.88$ $(\mathrm{d}, J=2.1 \mathrm{~Hz}, 1 \mathrm{H})$. The signals of the ${ }^{1} \mathrm{H}$ NMR spectra were in accordance with the data reported in the literature. ${ }^{36}$

1-(1-Bromovinyl)naphthalene (25p).

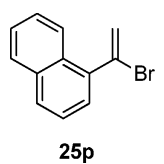

Compound 25p was prepared according to general method B, starting with 1-vinylnaphthalene ( $1.95 \mathrm{~g}, 12.7 \mathrm{mmol}, 1.0$ equiv), $\mathrm{Br}_{2}\left(78 \mu \mathrm{L}, 15 \mathrm{mmol}, 1.20\right.$ equiv), and $\mathrm{K}_{2} \mathrm{CO}_{3}(3.49 \mathrm{~g}, 25.3 \mathrm{mmol}$, 2.0 equiv). The title compound $25 \mathrm{p}$ was obtained as a colorless liquid (2.48 g, $10.6 \mathrm{mmol}, 84 \%$ yield). ${ }^{1} \mathrm{H}$ NMR (400 MHz, $\left.\mathrm{CDCl}_{3}, \delta\right): 8.23(\mathrm{dq}, J=8.5,0.9 \mathrm{~Hz}, 1 \mathrm{H}), 7.93-7.77(\mathrm{~m}, 2 \mathrm{H})$, 7.65-7.39 (m, 4H), $6.10(\mathrm{~d}, J=1.5 \mathrm{~Hz}, 1 \mathrm{H}), 5.97(\mathrm{~d}, J=1.5 \mathrm{~Hz}$, $1 \mathrm{H}) \mathrm{ppm}$. The signals of the ${ }^{1} \mathrm{H}$ NMR spectra were in accordance with the data reported in the literature. ${ }^{39}$

1-(1-Bromovinyl)-4-(trifluoromethyl)benzene (25t).

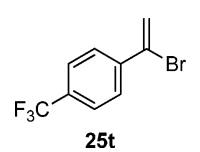


Compound 25t was prepared according to general method B, starting with 4-(trifluormethyl)-styrene $(3.4 \mathrm{~g}, 20 \mathrm{mmol}$, 1.0 equiv), $\mathrm{Br}_{2}$ (1.3 mL, $24 \mathrm{mmol}, 1.2$ equiv), and $\mathrm{K}_{2} \mathrm{CO}_{3}$ (5.5 g, $40 \mathrm{mmol}, 2.0$ equiv). The title compound 25t was obtained as a colorless liquid (2.2 g, $8.7 \mathrm{mmol}, 43 \%$ yield). ${ }^{1} \mathrm{H}$ NMR (400 MHz, $\left.\mathrm{CDCl}_{3}, \delta\right): 7.77-7.68(\mathrm{~m}, 2 \mathrm{H}), 7.61(\mathrm{~d}, J=$ $8.2 \mathrm{~Hz}, 2 \mathrm{H}), 6.21(\mathrm{~d}, J=2.2 \mathrm{~Hz}, 1 \mathrm{H}), 5.90(\mathrm{~d}, J=2.2 \mathrm{~Hz}$, $1 \mathrm{H})$ ppm. ${ }^{13} \mathrm{C}\left\{{ }^{1} \mathrm{H}\right\}$ NMR $\left(101 \mathrm{MHz}, \mathrm{CDCl}_{3}\right.$; the multiplet peak corresponding to the $\mathrm{CF}_{3}$ was not resolved, $\delta$ ): 141.9, 131.4, 131.0 (q, $J=32.7 \mathrm{~Hz}), 127.6,125.3(\mathrm{q}, J=3.9 \mathrm{~Hz}), 122.5,119.7 \mathrm{ppm}$. HRMS (ESI): $\left[\mathrm{M}^{+}\right]$calcd for $\mathrm{C}_{9} \mathrm{H}_{6} \mathrm{BrF}_{3}, 249.9599$; found, 249.9541. IR (film): 1612 (w), 1408 (w), 1327 (s), 1218 (w), $1170(\mathrm{~m}), 1130$ (s), 1075 (m), 1016 (w), 898 (w), 847 (m) cm $\mathrm{cm}^{-1}$.

Synthesis of 1-(1-(Arene)vinyl)cyclobutanols. General Method C. One crystal of iodine and 1,2-dibromoethane (0.4 equiv) were added to a suspension of magnesium (4.0 equiv) in THF $(0.2 \mathrm{~m})$. Thereafter, a solution of (1-bromovinyl)arene (1.3 equiv) in THF $(1 \mathrm{M})$ was added dropwise, and the reaction mixture was stirred for $1 \mathrm{~h}$ at $60^{\circ} \mathrm{C}$. Then, the corresponding cyclobutanone (1.0 equiv) was added dropwise, and stirring was continued at $60{ }^{\circ} \mathrm{C}$ for $5 \mathrm{~h}$. After being cooled to room temperature, the reaction was quenched with water and extracted with ethyl acetate $(3 \times 40 \mathrm{~mL})$. The combined organic extracts were washed with water $(50 \mathrm{~mL})$ and brine $(50 \mathrm{M})$ and then dried over $\mathrm{Na}_{2} \mathrm{SO}_{4}$, the drying agent was filtered off, and the solvent was evaporated. The residue was purified by column chromatography (silica; pentane:EtOAc 100:0 to 95:5 to $80: 20$ to $70: 30$ ), affording the title compound 3.

1-(1-Phenylvinyl)cyclobutanols (3a).<smiles>C=C(c1ccccc1)C1(O)CCC1</smiles>

Compound 3a was prepared according to general method $\mathrm{C}$ using magnesium ( $0.40 \mathrm{~g}, 16 \mathrm{mmol}, 4.0$ equiv), 1,2-dibromoethane (0.14 mL, $1.6 \mathrm{mmol}, 0.4$ equiv), (1-bromovinyl)benzene (0.70 mL, $5.2 \mathrm{mmol}$, 1.3 equiv., $90 \%$ purity), and cyclobutanone $(0.30 \mathrm{~mL}, 4.0 \mathrm{mmol}, 1.0$ equiv). The title compound 3a was obtained as a colorless oil $(0.65 \mathrm{~g}, 3.7 \mathrm{mmol}$, $93 \%$ yield). ${ }^{1} \mathrm{H}$ NMR (400 $\left.\mathrm{MHz}, \mathrm{CDCl}_{3}, \delta\right): 7.52-7.45$ (m, 2H), 7.38-7.27 (m, 3H), $5.38(\mathrm{~s}, 1 \mathrm{H}), 5.36(\mathrm{~d}, J=0.9 \mathrm{~Hz}$, $1 \mathrm{H}), 2.54-2.42(\mathrm{~m}, 2 \mathrm{H}), 2.25(\mathrm{~m}, 2 \mathrm{H}), 1.99(\mathrm{~m}, 2 \mathrm{H}), 1.63$ $(\mathrm{m}, 1 \mathrm{H})$ ppm. The signals of the ${ }^{1} \mathrm{H}$ NMR spectra were in accordance with the data reported in the literature. ${ }^{13 c}$

1-(1-(p-Tolyl)vinyl)cyclobutanol (3b).<smiles>C=C(c1ccc(C)cc1)C1(O)CCC1</smiles>

Compound $\mathbf{3 b}$ was prepared according to general method $\mathrm{C}$ using magnesium ( $0.30 \mathrm{~g}, 13 \mathrm{mmol}, 4.0$ equiv), 1,2-dibromoethane (0.10 mL, $1.3 \mathrm{mmol}, 0.4$ equiv), 1-(1-bromovinyl)-4-methylbenzene (25b, $1.0 \mathrm{~g}, 4.1 \mathrm{mmol}, 1.3$ equiv), and cyclobutanone ( $0.25 \mathrm{~mL}, 3.4 \mathrm{mmol}, 1.0$ equiv). The title compound $3 \mathrm{~b}$ was obtained as a colorless oil $(0.48 \mathrm{~g}, 2.5 \mathrm{mmol}, 75 \%$ yield $)$. ${ }^{1} \mathrm{H}$ NMR (400 MHz, $\mathrm{CDCl}_{3}, \delta$ ): 7.44-7.38 (m, 2H), 7.16 (d, $J=7.9 \mathrm{~Hz}, 2 \mathrm{H}), 5.34(\mathrm{~m}, 2 \mathrm{H}), 2.53-2.43(\mathrm{~m}, 2 \mathrm{H}), 2.37$ (s, 3H), $2.26(\mathrm{~m}, 3 \mathrm{H}), 2.01(\mathrm{~m}, 1 \mathrm{H}), 1.64(\mathrm{~m}, 1 \mathrm{H}) \mathrm{ppm}$. The signals of the ${ }^{1} \mathrm{H}$ NMR spectra were in accordance with the data reported in the literature. ${ }^{13 \mathrm{c}}$

1-(1-(4-(tert-Butyl)phenyl)vinyl)cyclobutanols (3c).

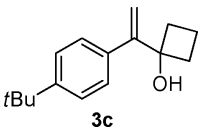

Compound $3 \mathrm{c}$ was prepared according to general method $\mathrm{C}$ using magnesium ( $0.30 \mathrm{~g}, 13 \mathrm{mmol}, 4.0$ equiv), 1,2-dibromoethane $(0.10 \mathrm{~mL}, 1.3 \mathrm{mmol}, 0.4$ equiv), 1-(1-bromovinyl)-4-(tertbutyl)benzene $(25 \mathrm{c}, 1.0 \mathrm{~g}, 4.2 \mathrm{mmol}, 1.3$ equiv), and cyclobutanone $(0.26 \mathrm{~mL}, 3.4 \mathrm{mmol}, 1.00$ equiv). The title compound $3 \mathrm{c}$ was obtained as a colorless oil $(0.46 \mathrm{~g}, 2.0 \mathrm{mmol}$, $53 \%$ yield). ${ }^{1} \mathrm{H}$ NMR (400 $\left.\mathrm{MHz}, \mathrm{CDCl}_{3}, \delta\right): 7.44-7.40$ $(\mathrm{m}, 2 \mathrm{H}), 7.37-7.34(\mathrm{~m}, 2 \mathrm{H}), 5.37(\mathrm{~d}, J=0.9 \mathrm{~Hz}, 1 \mathrm{H}), 5.34$ $(\mathrm{d}, J=1.0 \mathrm{~Hz}, 1 \mathrm{H}), 2.50(\mathrm{~m}, 2 \mathrm{H}), 2.27(\mathrm{~m}, 2 \mathrm{H}), 1.98(\mathrm{~m}$, $2 \mathrm{H}), 1.64(\mathrm{~m}, 1 \mathrm{H}), 1.32(\mathrm{~s}, 9 \mathrm{H}) \mathrm{ppm}$. The signals of the ${ }^{1} \mathrm{H}$ NMR spectra were in accordance with the data reported in the literature. ${ }^{13 c}$

1-(1-([1,1'-Biphenyl]-4-yl)vinyl)cyclobutanols (3d).<smiles>C=C(c1ccc(-c2ccccc2)cc1)C1(O)CCC1</smiles>

Compound 3d was prepared according to general method $\mathrm{C}$ using magnesium ( $0.25 \mathrm{~g}, 11 \mathrm{mmol}, 4.00$ equiv), 1,2-dibromoethane (0.10 mL, $1.1 \mathrm{mmol}, 0.4$ equiv), 4-(1-bromovinyl)-1,1'-biphenyl (25d, $0.85 \mathrm{~g}, 3.3 \mathrm{mmol}, 1.3$ equiv), and cyclobutanone $(0.21 \mathrm{~mL}$, $2.7 \mathrm{mmol}, 1.0$ equiv). The title compound $3 \mathrm{~d}$ was obtained as a colorless oil (0.56 g, $2.7 \mathrm{mmol}, 53 \%$ yield). ${ }^{1} \mathrm{H}$ NMR $\left(400 \mathrm{MHz}, \mathrm{CDCl}_{3}, \delta\right): 7.64-7.53(\mathrm{~m}, 5 \mathrm{H}), 7.45(\mathrm{~m}, 2 \mathrm{H}), 7.35$ $(\mathrm{m}, 1 \mathrm{H}), 5.45(\mathrm{~d}, J=0.8 \mathrm{~Hz}, 1 \mathrm{H}), 5.41(\mathrm{~d}, J=0.8 \mathrm{~Hz}, 1 \mathrm{H})$, $2.52(\mathrm{~m}, 2 \mathrm{H}), 2.29(\mathrm{~m}, 2 \mathrm{H}), 2.02(\mathrm{~m}, 2 \mathrm{H}), 1.67(\mathrm{~m}, 1 \mathrm{H}) \mathrm{ppm}$. The signals of the ${ }^{1} \mathrm{H}$ NMR spectra were in accordance with the data reported in the literature. ${ }^{14 a}$

1-(1-(4-Methoxyphenyl)vinyl)cyclobutanols (3e).

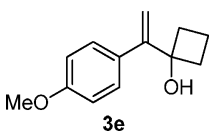

Compound $3 \mathbf{e}$ was prepared according to general method $\mathrm{C}$ using magnesium ( $0.380 \mathrm{~g}, 15.6 \mathrm{mmol}, 4.00 \mathrm{equiv}), 1,2-$ dibromoethane $(0.13 \mathrm{~mL}, 1.6 \mathrm{mmol}, 0.40$ equiv), 1-(1bromovinyl)-4-methoxybenzene (25e, $1.0 \mathrm{~g}, 4.7 \mathrm{mmol}$, 1.3 equiv), and cyclobutanone ( $0.29 \mathrm{~mL}, 3.9 \mathrm{mmol}, 1.00$ equiv). The title compound $3 \mathrm{e}$ was obtained as a colorless oil $(0.560 \mathrm{~g}$, $2.74 \mathrm{mmol}, 70 \%$ yield). ${ }^{1} \mathrm{H} \mathrm{NMR}\left(400 \mathrm{MHz}, \mathrm{CDCl}_{3}, \delta\right): 7.44$ $(\mathrm{m}, 2 \mathrm{H}), 6.86(\mathrm{~m}, 2 \mathrm{H}), 5.31(\mathrm{~d}, J=0.9 \mathrm{~Hz}, 1 \mathrm{H}), 5.30(\mathrm{~d}, J=$ $0.9 \mathrm{~Hz}, 1 \mathrm{H}), 3.81(\mathrm{~s}, 3 \mathrm{H}), 2.47(\mathrm{~m}, 2 \mathrm{H}), 2.25(\mathrm{~m}, 2 \mathrm{H}), 1.98$ $(\mathrm{m}, 2 \mathrm{H}), 1.63(\mathrm{~m}, 1 \mathrm{H}) \mathrm{ppm}$. The signals of the ${ }^{1} \mathrm{H}$ NMR spectra were in accordance with the data reported in the literature. $^{14 \mathrm{a}}$

1-(1-(4-Fluorophenyl)vinyl)cyclobutanols (3f).

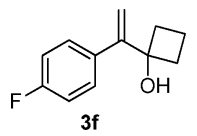

Compound $3 \mathrm{f}$ was prepared according to general method $\mathrm{C}$ using magnesium (0.40 g, $16 \mathrm{mmol}, 4.0$ equiv), 1,2dibromoethane $(0.20 \mathrm{~mL}, 2.6 \mathrm{mmol}, 0.4$ equiv $), 1-(1-$ bromovinyl)-4-fluorobenzene (25f, $1.6 \mathrm{~g}, 8.0 \mathrm{mmol}$, 1.3 equiv), and cyclobutanone $(0.50 \mathrm{~mL}, 6.6 \mathrm{mmol}, 1.0$ equiv $)$. The title compound $3 \mathbf{f}$ was obtained as a colorless oil $(0.95 \mathrm{~g}, 4.9 \mathrm{mmol}$, $74 \%$ yield). ${ }^{1} \mathrm{H}$ NMR (400 $\left.\mathrm{MHz}, \mathrm{CDCl}_{3}, \delta\right): 7.52-7.41$ (m, 2H), 7.13-6.91 (m, 2H), $5.36(\mathrm{~d}, J=0.8 \mathrm{~Hz}, 1 \mathrm{H}), 5.32$ $(\mathrm{d}, J=0.7 \mathrm{~Hz}, 1 \mathrm{H}), 2.44(\mathrm{~m}, 2 \mathrm{H}), 2.22(\mathrm{~m}, 2 \mathrm{H}), 1.99$ $(\mathrm{m}, 1 \mathrm{H}), 1.90(\mathrm{~m}, 1 \mathrm{H}), 1.62(\mathrm{~m}, 1 \mathrm{H}) \mathrm{ppm}$. The signals of the ${ }^{1} \mathrm{H}$ NMR spectra were in accordance with the data reported in the literature. ${ }^{13 c}$ 
1-(1-(4-Chlorophenyl)vinyl)cyclobutanols (3g).<smiles>C=C(c1ccc(Cl)cc1)C1(O)CCC1</smiles>

Compound $3 \mathrm{~g}$ was prepared according to general method $\mathrm{C}$ using magnesium ( $0.30 \mathrm{~g}, 12 \mathrm{mmol}, 4.0$ equiv), 1,2-dibromoethane (0.11 mL, $1.3 \mathrm{mmol}, 0.4$ equiv), 1-(1-bromovinyl)-4-chlorobenzene (25g, $0.83 \mathrm{~g}$, $3.8 \mathrm{mmol}, 1.3$ equiv), and cyclobutanone ( $0.24 \mathrm{~mL}, 3.2 \mathrm{mmol}, 1.0$ equiv). The title compound $3 \mathrm{~g}$ was obtained as a colorless oil ( $0.42 \mathrm{~g}, 2.0 \mathrm{mmol}, 64 \%$ yield). ${ }^{1} \mathrm{H} \mathrm{NMR}\left(400 \mathrm{MHz}, \mathrm{CDCl}_{3}, \delta\right): 7.46-7.39$ (m, 2H), 7.31-7.27 (m, 2H), $5.38(\mathrm{~s}, 1 \mathrm{H}), 5.36(\mathrm{~d}, J=0.7 \mathrm{~Hz}, 1 \mathrm{H}), 2.44(\mathrm{~m}, 2 \mathrm{H})$, $2.22(\mathrm{~m}, 2 \mathrm{H}), 1.99(\mathrm{~m}, 1 \mathrm{H}), 1.86$ (brs, $1 \mathrm{H}), 1.62(\mathrm{~m}, 1 \mathrm{H}) \mathrm{ppm}$. The signals of the ${ }^{1} \mathrm{H}$ NMR spectra were in accordance with the data reported in the literature. ${ }^{13 c}$

1-(1-(m-Tolyl)vinyl)cyclobutanol (3h).

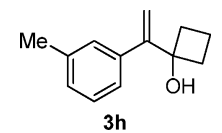

Compound $3 \mathrm{~h}$ was prepared according to general method C using magnesium (0.410 g, $16.9 \mathrm{mmol}, 4.00$ equiv), 1,2-dibromoethane $(0.14 \mathrm{~mL}, 1.7 \mathrm{mmol}, 0.40$ equiv), 1-(1bromovinyl)-3-methylbenzene ( $25 \mathrm{~h}, 1.0 \mathrm{~g}, 5.1 \mathrm{mmol}, 1.3 \mathrm{equiv})$, and cyclobutanone $(0.32 \mathrm{~mL}, 4.23 \mathrm{mmol}, 1.0$ equiv). The title compound $3 \mathrm{~h}$ was obtained as a colorless oil $(0.680 \mathrm{~g}$, $3.61 \mathrm{mmol}, 85 \%$ yield). ${ }^{1} \mathrm{H} \mathrm{NMR}\left(400 \mathrm{MHz}, \mathrm{CDCl}_{3}, \delta\right): 7.27$ $(\mathrm{m}, 2 \mathrm{H}), 7.22(\mathrm{td}, J=7.3,1.3 \mathrm{~Hz}, 1 \mathrm{H}), 7.12(\mathrm{~m}, 1 \mathrm{H}), 5.36$ $(\mathrm{d}, J=0.9 \mathrm{~Hz}, 1 \mathrm{H}), 5.34(\mathrm{~d}, J=0.9 \mathrm{~Hz}, 1 \mathrm{H}), 2.48(\mathrm{~m}, 2 \mathrm{H})$, $2.36(\mathrm{~s}, 3 \mathrm{H}), 2.25(\mathrm{~m}, 2 \mathrm{H}), 1.99(\mathrm{~m}, 2 \mathrm{H}), 1.63(\mathrm{~m}, 1 \mathrm{H}) \mathrm{ppm}$. The signals of the ${ }^{1} \mathrm{H}$ NMR spectra were in accordance with the data reported in the literature. ${ }^{13 c}$

1-(1-(3-Methoxyphenyl)vinyl)cyclobutanol (3i).

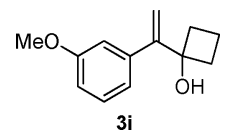

Compound $3 \mathbf{i}$ was prepared according to the general method $\mathrm{C}$ using magnesium (0.140 g, $5.63 \mathrm{mmol}, 4.00$ equiv), 1,2-dibromoethane ( $50 \mu \mathrm{L}, 0.60 \mathrm{mmol}, 0.40$ equiv), 1-(1bromovinyl)-3-methoxybenzene (25i, $0.36 \mathrm{~g}, 1.67 \mathrm{mmol}$, 1.3 equiv), and cyclobutanone ( $0.11 \mathrm{~mL}, 1.4 \mathrm{mmol}, 1.0$ equiv). The title compound $3 \mathbf{i}$ was obtained as a colorless oil $(0.15 \mathrm{~g}$, $0.73 \mathrm{mmol}, 52 \%$ yield). $R_{f}$ (silica, pentane:EtOAc 4:1): 0.4 . ${ }^{1} \mathrm{H}$ NMR (400 MHz, $\left.\mathrm{CDCl}_{3}, \delta\right): 7.27(\mathrm{t}, J=8.0 \mathrm{~Hz}, 1 \mathrm{H})$, $7.14-7.03(\mathrm{~m}, 2 \mathrm{H}), 6.87$ (ddd, $J=8.2,2.6,1.0 \mathrm{~Hz}, 1 \mathrm{H}), 5.39$ (d, $J=2.3 \mathrm{~Hz}, 1 \mathrm{H}), 3.83(\mathrm{~s}, 3 \mathrm{H}), 2.56-2.44(\mathrm{~m}, 2 \mathrm{H}), 2.35$ $(\mathrm{t}, J=3.8 \mathrm{~Hz}, 1 \mathrm{H}), 2.33-2.20(\mathrm{~m}, 2 \mathrm{H}), 2.09-1.91(\mathrm{~m}, 1 \mathrm{H})$, $1.65(\mathrm{dtt}, J=11.0,8.8,6.8 \mathrm{~Hz}, 1 \mathrm{H})$. The ${ }^{1} \mathrm{H}$ NMR data correspond to the reported values. ${ }^{12 \mathrm{f}}$

1-(1-(3-Fluorophenyl)vinyl)cyclobutanols (3j).

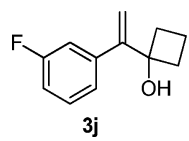

Compound $3 \mathbf{j}$ was prepared according to general method $\mathrm{C}$ using magnesium (0.400 g, $16.5 \mathrm{mmol}, 4.00$ equiv), 1,2-dibromoethane ( $0.23 \mathrm{~mL}, 2.7 \mathrm{mmol}, 0.40$ equiv), 1-(1bromovinyl)-3-fluorobenzene $(\mathbf{2 5 j}, 1.6 \mathrm{~g}, 8.0 \mathrm{mmol}, 1.3$ equiv), and cyclobutanone $(0.50 \mathrm{~mL}, 6.6 \mathrm{mmol}, 1.0$ equiv). The title compound $3 \mathbf{j}$ was obtained as a colorless oil (0.670 g, $3.49 \mathrm{mmol}$, 52\% yield). ${ }^{1} \mathrm{H}$ NMR (400 $\left.\mathrm{MHz}, \mathrm{CDCl}_{3}, \delta\right)$ : $7.31-7.12(\mathrm{~m}, 3 \mathrm{H}), 6.95(\mathrm{~m}, 1 \mathrm{H}), 5.37(\mathrm{~s}, 1 \mathrm{H}), 5.36(\mathrm{~s}, 1 \mathrm{H})$, $2.42(\mathrm{~m}, 2 \mathrm{H}), 2.20(\mathrm{~m}, 2 \mathrm{H}), 1.97(\mathrm{~m}, 1 \mathrm{H}), 1.92$ (brs, $1 \mathrm{H})$, $1.59(\mathrm{~m}, 1 \mathrm{H}) \mathrm{ppm}$. The ${ }^{1} \mathrm{H}$ NMR data correspond to the reported values, with a systematic shift of $0.15 \mathrm{ppm}^{13 \mathrm{c}}$

\section{1-(1-(o-Tolyl)vinyl)cyclobutanol (3k).}

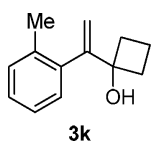

A solution of $t \mathrm{BuLi}(6.18 \mathrm{~mL}, 12.4 \mathrm{mmol}, 2 \mathrm{~m}$ in hexane, 2 equiv) was added at $-78{ }^{\circ} \mathrm{C}$ to a solution of $25 \mathrm{k}(1.22 \mathrm{~g}$, $6.19 \mathrm{mmol}, 1$ equiv) in THF $(25 \mathrm{~mL}, 0.25 \mathrm{~m})$, and the resulting mixture was stirred for $1.5 \mathrm{~h}$ at $-78{ }^{\circ} \mathrm{C}$. Thereafter, cyclobutanone ( $0.59 \mathrm{~mL} 8.1 \mathrm{mmol}, 1.3$ equiv) was added dropwise, and stirring was continued for $1 \mathrm{~h}$ at $-78{ }^{\circ} \mathrm{C}$. Then, the reaction was quenched with water $(30 \mathrm{~mL})$, warmed to room temperature, and extracted with $\mathrm{Et}_{2} \mathrm{O}(3 \times 30 \mathrm{~mL})$. The combined organic extracts were washed with brine $(20 \mathrm{~mL})$ and dried over $\mathrm{MgSO}_{4}$. The drying agent was filtered off, and the solvent was evaporated. The residue was purified by column chromatography (silica, pentane:EtOAc 1:0 to 10:1) affording $336 \mathrm{mg}$ (1.79 mmol, 29\%) of the title compound $3 \mathrm{k}$ as a colorless oil. ${ }^{1} \mathrm{H} \mathrm{NMR}\left(400 \mathrm{MHz}, \mathrm{CDCl}_{3}, \delta\right): 7.57-6.90$ $(\mathrm{m}, 4 \mathrm{H}), 5.53(\mathrm{t}, J=1.5 \mathrm{~Hz}, 1 \mathrm{H}), 4.99(\mathrm{t}, J=1.6 \mathrm{~Hz}, 1 \mathrm{H})$, $2.52-2.36(\mathrm{~m}, 2 \mathrm{H}), 2.28(\mathrm{~s}, 3 \mathrm{H}), 2.17-2.02(\mathrm{~m}, 2 \mathrm{H}), 2.02-$ $1.76(\mathrm{~m}, 2 \mathrm{H}), 1.66-1.45(\mathrm{~m}, 1 \mathrm{H})$ ppm. The signals of the ${ }^{1} \mathrm{H}$ NMR spectra were in accordance with the data reported in the literature. ${ }^{13 c}$

1-(1-(3,4-Dimethylphenyl)vinyl)cyclobutanols (3l).<smiles>C=C(c1cccc(C)c1)C1(O)CCC1</smiles>

Compound 31 was prepared according to general method $\mathrm{C}$ using magnesium (0.450 g, $18.4 \mathrm{mmol}, 4.00$ equiv), 1,2-dibromoethane $(0.16 \mathrm{~mL}, 1.8 \mathrm{mmol}, 0.400$ equiv), 4-(1bromovinyl)-1,2-dimethylbenzene (25l, $1.26 \mathrm{~g}, 5.97 \mathrm{mmol}$, 1.30 equiv), and cyclobutanone ( $0.34 \mathrm{~mL}, 4.6 \mathrm{mmol}, 1.0$ equiv). The title compound 31 was obtained as a yellow oil $(0.590 \mathrm{~g}$, $2.91 \mathrm{mmol}, 63 \%$ yield). $R_{f}$ (silica, pentane): $0.5 .{ }^{1} \mathrm{H}$ NMR $\left(400 \mathrm{MHz}, \mathrm{CDCl}_{3}, \delta\right): 7.27-7.23(\mathrm{~m}, 1 \mathrm{H}), \quad 7.23-7.18$ $(\mathrm{m}, 1 \mathrm{H}), 7.09(\mathrm{~d}, J=7.8 \mathrm{~Hz}, 1 \mathrm{H}), 5.40-5.17(\mathrm{~m}, 2 \mathrm{H})$, $2.58-2.42(\mathrm{~m}, 2 \mathrm{H}), 2.34-2.18(\mathrm{~m}, 8 \mathrm{H}), 2.11-1.86(\mathrm{~m}, 2 \mathrm{H})$, $1.72-1.55(\mathrm{~m}, 1 \mathrm{H}) \mathrm{ppm} .{ }^{13} \mathrm{C}\left\{{ }^{1} \mathrm{H}\right\} \mathrm{NMR}\left(101 \mathrm{MHz}, \mathrm{CDCl}_{3}, \delta\right)$ : $152.3,136.5,136.3,136.0,129.4,128.8,125.0,112.0,78.1$, 35.7, 19.9, 19.4, $13.4 \mathrm{ppm}$. HRMS (ESI): $\left[\mathrm{M}^{+}\right]$calcd for $\mathrm{C}_{14} \mathrm{H}_{18} \mathrm{O}, 202.1352$; found, 202.1354. IR (film): $3398(\mathrm{~m})$, $2986(\mathrm{~m}), 2940(\mathrm{~s}), 2871(\mathrm{w}), 1624(\mathrm{w}), 1503(\mathrm{~m}), 1451(\mathrm{~m})$, $1383(\mathrm{w}), 1250(\mathrm{~m}), 1225(\mathrm{w}), 1153(\mathrm{~m}), 1111(\mathrm{~m}), 1078(\mathrm{w})$, $1022(\mathrm{w}), 908(\mathrm{~s}), 826(\mathrm{~s}) \mathrm{cm}^{-1}$.

1-(1-(3,4-Dimethoxyphenyl)vinyl)cyclobutanol (3m).

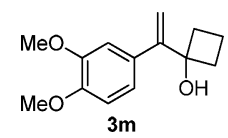

Compound $3 \mathrm{~m}$ was prepared according to general method $\mathrm{C}$ using magnesium ( $0.190 \mathrm{~g}, 7.95 \mathrm{mmol}, 4.00$ equiv), 1,2-dibromoethane (70 $\mu \mathrm{L}, 0.79 \mathrm{mmol}, 0.40$ equiv), 4-(1bromovinyl)-1,2-dimethoxybenzene $(25 \mathrm{~m}, 0.580 \mathrm{~g}, 2.39 \mathrm{mmol}$, 1.30 equiv), and cyclobutanone ( $0.15 \mathrm{~mL}, 2.0 \mathrm{mmol}, 1.00$ equiv). 
The title compound $3 \mathrm{~m}$ was obtained as a colorless oil $(0.20 \mathrm{~g}$, $0.85 \mathrm{mmol}, 62 \%$ yield). $R_{f}$ (silica, pentane:EtOAc 4:1): 0.2 . ${ }^{1} \mathrm{H}$ NMR $\left(400 \mathrm{MHz}, \mathrm{CDCl}_{3}, \delta\right): 7.02(\mathrm{~m}, 2 \mathrm{H}), 6.78(\mathrm{~d}, J=$ $8.1 \mathrm{~Hz}, 1 \mathrm{H}), 5.28(\mathrm{~d}, J=0.9 \mathrm{~Hz}, 1 \mathrm{H}), 5.26(\mathrm{~d}, J=0.9 \mathrm{~Hz}, 1 \mathrm{H})$, $3.84(\mathrm{~s}, 6 \mathrm{H}), 2.43(\mathrm{~m}, 2 \mathrm{H}), 2.35-2.16(\mathrm{~m}, 3 \mathrm{H}), 1.95(\mathrm{~m}, 1 \mathrm{H})$, $1.59(\mathrm{~m}, 1 \mathrm{H}) \mathrm{ppm} .{ }^{13} \mathrm{C}\left\{{ }^{1} \mathrm{H}\right\}$ NMR $\left(101 \mathrm{MHz}, \mathrm{CDCl}_{3}, \delta\right)$ : $151.8,148.5,148.4,131.7,119.9,111.7,110.9,110.7,78.2$, $55.8,35.6,13.4 \mathrm{ppm}$. One alkyl signal was not resolved. HRMS (ESI): $[\mathrm{M}+\mathrm{H}]^{+}$calcd. for $\mathrm{C}_{14} \mathrm{H}_{19} \mathrm{O}_{3}{ }^{+}, 235.1329$; found, 235.1329. IR (film): 3484 (w), 2986 (w), 2937 (w), 2912 (w), $2835(\mathrm{w}), 2361(\mathrm{w}), 2342(\mathrm{w}), 2254(\mathrm{w}), 1514(\mathrm{~s}), 1254(\mathrm{~s})$, 1143 (s), 1027 (s), 909 (s) $\mathrm{cm}^{-1}$.

1-(1-(Benzo[d][1,3]dioxol-5-yl)vinyl)cyclobutanol (3n).

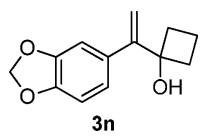

Compound 3n was prepared according to general method C using magnesium (0.530 g, $22.0 \mathrm{mmol}, 4.00$ equiv), 1,2-dibromoethane $(0.19 \mathrm{~mL}, 2.0 \mathrm{mmol}, 0.40$ equiv), 5-(1bromovinyl)benzo[d][1,3] dioxole $(25 \mathrm{n}, 1.5 \mathrm{~g}, 6.6 \mathrm{mmol}$, 1.3 equiv), and cyclobutanone ( $0.41 \mathrm{~mL}, 5.5 \mathrm{mmol}, 1.00$ equiv). The title compound $3 \mathbf{n}$ was obtained as a colorless oil $(0.560 \mathrm{~g}$, $2.57 \mathrm{mmol}, 47 \%$ yield ). ${ }^{1} \mathrm{H}$ NMR (400 $\mathrm{MHz}, \mathrm{CDCl}_{3}, \delta$ ): 7.01 $(\mathrm{d}, J=1.8 \mathrm{~Hz}, 1 \mathrm{H}), 6.97(\mathrm{dd}, J=8.1,1.7 \mathrm{~Hz}, 1 \mathrm{H}), 6.77(\mathrm{dt}, J=$ 8.1, $0.9 \mathrm{~Hz}, 1 \mathrm{H}), 5.95\left(\mathrm{~m}, 2 \mathrm{H}, \mathrm{OCH}_{2} \mathrm{O}\right), 5.29(\mathrm{~m}, 2 \mathrm{H}), 2.45$ $(\mathrm{m}, 2 \mathrm{H}), 2.22(\mathrm{~m}, 2 \mathrm{H}), 1.97(\mathrm{~m}, 2 \mathrm{H}), 1.61(\mathrm{~m}, 1 \mathrm{H}) \mathrm{ppm}$. The signals of the ${ }^{1} \mathrm{H}$ NMR spectra were in accordance with the data reported in the literature. ${ }^{14 a}$

1-(1-(Naphthalen-2-yl)vinyl)cyclobutanol (30).

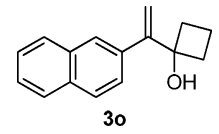

Compound 3o was prepared according to general method C using magnesium ( $0.35 \mathrm{~g}, 14.3 \mathrm{mmol}, 4.00$ equiv), 1,2-dibromoethane $(0.12 \mathrm{~mL}, 1.4 \mathrm{mmol}, 0.40$ equiv), 2-(1bromovinyl)naphthalene (25o, $1.0 \mathrm{~g}, 4.3 \mathrm{mmol}, 1.3 \mathrm{equiv})$, and cyclobutanone $(0.27 \mathrm{~mL}, 3.6 \mathrm{mmol}, 1.0$ equiv). The title compound $3 \mathrm{o}$ was obtained as a colorless oil $(0.50 \mathrm{~g}$, $2.2 \mathrm{mmol}, 62 \%$ yield). ${ }^{1} \mathrm{H} \mathrm{NMR}\left(400 \mathrm{MHz}, \mathrm{CDCl}_{3}, \delta\right.$ ): 7.96 $(\mathrm{m}, 1 \mathrm{H}), 7.87-7.76(\mathrm{~m}, 3 \mathrm{H}), 7.63(\mathrm{dd}, J=8.5,1.8 \mathrm{~Hz}, 1 \mathrm{H})$, $7.47(\mathrm{~m}, 2 \mathrm{H}), 5.50(\mathrm{~d}, J=0.8 \mathrm{~Hz}, 1 \mathrm{H}), 5.48(\mathrm{~d}, J=0.8 \mathrm{~Hz}$, $1 \mathrm{H}), 2.53(\mathrm{~m}, 2 \mathrm{H}), 2.31(\mathrm{~m}, 2 \mathrm{H}), 2.02(\mathrm{~m}, 2 \mathrm{H}), 1.67(\mathrm{~m}, 1 \mathrm{H})$ ppm. ${ }^{1} \mathrm{H}$ NMR data correspond to the reported values, apart from the peak at $\delta=1.67 \mathrm{ppm}$, which corresponds better to the attributed ${ }^{1} \mathrm{H}$ nuclei than the signal in the reference article. $^{14 \mathrm{a}}$

1-(1-(Naphthalen-1-yl)vinyl)cyclobutanol (3p).

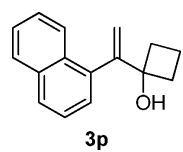

Compound $3 \mathrm{p}$ was prepared according to general method $\mathrm{C}$ using magnesium (796 mg, $32.7 \mathrm{mmol}, 4.00$ equiv), 1,2-dibromoethane ( $0.28 \mathrm{~mL}, 3.3 \mathrm{mmol}, 0.40$ equiv), $25 \mathrm{p}$ (2.48 g, $10.6 \mathrm{mmol}, 1.3$ equiv), and cyclobutanone $(0.60 \mathrm{~mL}$, $8.2 \mathrm{mmol}, 1.0$ equiv). The title compound $3 \mathrm{p}$ was obtained as a colorless oil (1.01 g, $4.48 \mathrm{mmol}, 55 \%$ yield). $R_{f}$ (silica, pentane:EtOAc 10:1): 0.5. ${ }^{1} \mathrm{H}$ NMR (400 $\left.\mathrm{MHz}, \mathrm{CDCl}_{3}, \delta\right)$ : 8.09-8.02 (m, 1H), 7.89-7.84 (m, 1H), 7.84-7.78 (m, 1H),
7.53-7.42 (m, 3H), $7.40(\mathrm{dt}, J=7.0,1.0 \mathrm{~Hz}, 1 \mathrm{H}), 5.78(\mathrm{~d}, J=$ $1.4 \mathrm{~Hz}, 1 \mathrm{H}), 5.19$ (d, $J=1.4 \mathrm{~Hz}, 1 \mathrm{H}), 2.54-2.37(\mathrm{~m}, 2 \mathrm{H})$, 2.21-2.04 (m, 2H), 2.04-1.85 (m, 2H), 1.68-1.50 (m, $1 \mathrm{H})$ ppm. ${ }^{13} \mathrm{C}\left\{{ }^{1} \mathrm{H}\right\}$ NMR $\left(101 \mathrm{MHz}, \mathrm{CDCl}_{3}, \delta\right): 151.1,137.9$, $133.6,132.5,128.1,127.6,126.3,126.1,125.9,125.7,124.9$, $115.2,78.5,35.3,13.5 \mathrm{ppm}$. HRMS (ESI): $\left[\mathrm{M}^{+}\right]$calcd for $\mathrm{C}_{16} \mathrm{H}_{16} \mathrm{O}, 224.1196$; found, 224.1201. IR (film): $3390(\mathrm{w})$, $3043(w), 2985(w), 2946(w), 1591(w), 1506(w), 1462(w)$, $1249(\mathrm{w}), 1156(\mathrm{w}), 1122(\mathrm{w}), 913(\mathrm{~m}), 804(\mathrm{~m}), 779(\mathrm{~s}) \mathrm{cm}^{-1}$. 3-(1-Phenylvinyl)oxetan-3-ol (3q).

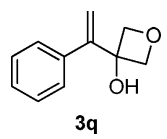

Compound $3 \mathbf{q}$ was prepared according to general method C using magnesium (0.400 g, $16.4 \mathrm{mmol}, 4.00$ equiv), 1,2-dibromoethane $(0.14 \mathrm{~mL}, 1.6 \mathrm{mmol}, 0.40$ equiv $)$, (1-bromovinyl)benzene $(0.71 \mathrm{~mL}, 4.9 \mathrm{mmol}, 1.3$ equiv., $90 \%$ purity), and oxetan-3-one ( $0.29 \mathrm{~mL}, 4.1 \mathrm{mmol}, 1.0$ equiv). The title compound $3 \mathrm{q}$ was obtained as a colorless oil $(0.41 \mathrm{~g}$, $2.3 \mathrm{mmol}, 57 \%$ yield). ${ }^{1} \mathrm{H} \mathrm{NMR}\left(400 \mathrm{MHz}, \mathrm{CDCl}_{3}, \delta\right): 7.42-$ $7.29(\mathrm{~m}, 5 \mathrm{H}), 5.59(\mathrm{~s}, 1 \mathrm{H}), 5.41(\mathrm{~s}, 1 \mathrm{H}), 4.93(\mathrm{dd}, J=6.9$, $0.9 \mathrm{~Hz}, 2 \mathrm{H}), 4.80(\mathrm{dd}, J=6.9,0.9 \mathrm{~Hz}, 2 \mathrm{H}), 2.57$ (s, $1 \mathrm{H}) \mathrm{ppm}$. The ${ }^{1} \mathrm{H}$ NMR data correspond to the reported values, with a systematic shift of $0.15 \mathrm{ppm}^{13 \mathrm{c}}$

tert-Butyl 3-Hydroxy-3-(1-phenylvinyl)azetidine-1-carboxylate (3r).

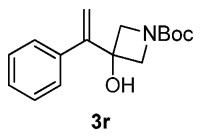

Compound $3 \mathbf{r}$ was prepared according to general method C using magnesium (227 mg, $9.35 \mathrm{mmol}, 4.00$ equiv), 1,2-dibromoethane $(0.08 \mathrm{~mL}, 0.09 \mathrm{mmol}, 0.40$ equiv), (1-bromovinyl)benzene $(0.40 \mathrm{~mL}, 2.8 \mathrm{mmol}, 1.2$ equiv), and tert-butyl 3-oxoazetidine-1-carboxylate $(0.40 \mathrm{~g}, 2.3 \mathrm{mmol}$, 1.00 equiv). The title compound $3 \mathbf{r}$ was obtained as a colorless solid (95\% pure; $0.46 \mathrm{~g}, 1.7 \mathrm{mmol}, 71 \%$ yield). mp 95$110{ }^{\circ} \mathrm{C} .{ }^{1} \mathrm{H}$ NMR $\left(400 \mathrm{MHz}, \mathrm{CDCl}_{3}, \delta\right): 7.45-7.38(\mathrm{~m}, 2 \mathrm{H})$, $7.38-7.28(\mathrm{~m}, 3 \mathrm{H}), 5.50(\mathrm{~s}, 1 \mathrm{H}), 5.39(\mathrm{~s}, 1 \mathrm{H}), 4.20(\mathrm{~d}, J=9.3$, $2 \mathrm{H}), 4.03(\mathrm{dd}, J=9.3,1.1,2 \mathrm{H}), 1.41(\mathrm{~s}, 9 \mathrm{H}) \mathrm{ppm} .{ }^{13} \mathrm{C}\left\{{ }^{1} \mathrm{H}\right\}$ NMR (101 MHz, $\left.\mathrm{CDCl}_{3}, \delta\right): 156.4,149.5,137.7,128.5,128.0$, 126.9, 114.8, 79.7, 72.5, 69.9, 61.9, 28.3 ppm. HRMS (ESI): [M + $\mathrm{Na}]^{+}$calcd for $\mathrm{C}_{16} \mathrm{H}_{21} \mathrm{NNaO}_{3}{ }^{+}, 298.1414$; found, 298.1413 .

1-(1-(4-(Trifluoromethyl)phenyl)vinyl)cyclobutanols (3t).

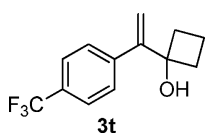

Compound 3t was prepared according to general method $\mathrm{C}$ using magnesium ( $843 \mathrm{mg}, 34.7 \mathrm{mmol}, 4.00$ equiv), 1,2-dibromoethane $(0.30 \mathrm{~mL}, 3.5 \mathrm{mmol}, 0.40$ equiv), $25 \mathrm{t}$ (2.83 g, $11.3 \mathrm{mmol}, 1.30$ equiv), and cyclobutanone ( $0.64 \mathrm{~mL}$, $8.7 \mathrm{mmol}, 1.00$ equiv). The title compound $3 \mathrm{t}$ was obtained as a colorless oil (1.01 g, $4.16 \mathrm{mmol}, 48 \%$ yield). $R_{f}$ (silica, pentane:EtOAc 10:1): 0.5. ${ }^{1} \mathrm{H}$ NMR (400 MHz, $\left.\mathrm{CDCl}_{3}, \delta\right)$ : $7.72-7.44(\mathrm{~m}, 4 \mathrm{H}), 5.46(\mathrm{~s}, 1 \mathrm{H}), 5.42(\mathrm{~s}, 1 \mathrm{H}), 2.49-2.39$ (m, 2H), 2.29-2.18 (m, 2H), 2.08-1.94 (m, 2H), 1.70-1.55 $(\mathrm{m}, 1 \mathrm{H})$ ppm. ${ }^{13} \mathrm{C}\left\{{ }^{1} \mathrm{H}\right\}$ NMR $\left(101 \mathrm{MHz}, \mathrm{CDCl}_{3}, \delta\right): 151.4$, $142.8,129.4(\mathrm{q}, J=32.4 \mathrm{~Hz}), 127.9,125.0(\mathrm{q}, J=3.7 \mathrm{~Hz})$, $124.2(\mathrm{q}, J=271.4 \mathrm{~Hz}), 114.5,77.9,35.6,13.3$ ppm. HRMS (ESI): $\left[\mathrm{M}^{+}\right]$calcd for $\mathrm{C}_{13} \mathrm{H}_{13} \mathrm{~F}_{3} \mathrm{O}, 242.0913$; found, 242.0917. 
IR (film): 3381 (w), 2991 (w), 2953 (w), 1619 (w), 1573 (w), $1407(\mathrm{w}), 1326(\mathrm{~s}), 1251(\mathrm{w}), 1166(\mathrm{~m}), 1122(\mathrm{~s}), 1067(\mathrm{~m})$, $1018(\mathrm{~m}), 912(\mathrm{~m}), 850(\mathrm{~m}) \mathrm{cm}^{-1}$.

Synthesis of (1-(1-(Arene)vinyl)cyclobutoxy)trimethylsilanes. General Procedure D. TMSCl (1.2 equiv) was added dropwise to a solution of (1-(arene)vinyl)cyclobutanol 3 (1.0 equiv) and triethylamine (1.5 equiv) in DCM $(0.2 \mathrm{M})$ at $0{ }^{\circ} \mathrm{C}$. The reaction mixture was stirred until full conversion on TLC, then it was quenched with sat. aq. $\mathrm{NH}_{4} \mathrm{Cl}(10 \mathrm{~mL})$, and extracted with $\mathrm{CH}_{2} \mathrm{Cl}_{2}(3 \times 30 \mathrm{~mL})$. The combined organic extracts were washed with brine and dried over $\mathrm{MgSO}_{4}$, the drying agent was filtered off, and the solvent was evaporated. Purification of the residue by column chromatography (silica, pentane:EtOAc 1:0 to 97:3) afforded the title product 4 .

Trimethyl(1-(1-phenylvinyl)cyclobutoxy)silane (4a).<smiles>C=C(c1ccccc1)C1(OC)CCC1</smiles>

Compound 4a was prepared according to general method $\mathrm{D}$ using 1-(1-phenylvinyl)cyclobutanol (3a, $1.58 \mathrm{~g}, 9.07 \mathrm{mmol}$, 1.00 equiv), $\mathrm{Et}_{3} \mathrm{~N}$ ( $0.90 \mathrm{~mL}, 13 \mathrm{mmol}, 1.5$ equiv), and $\mathrm{TMSCl}$ ( $1.4 \mathrm{~mL}, 11 \mathrm{mmol}, 1.3$ equiv). The title compound $4 \mathrm{a}$ was obtained as a colorless oil (2.0 g, $8.1 \mathrm{mmol}, 90 \%$ yield $)$. $R_{f}$ (silica, pentane): 0.6. ${ }^{1} \mathrm{H}$ NMR $\left(400 \mathrm{MHz}, \mathrm{CDCl}_{3}, \delta\right): 7.53$ $(\mathrm{m}, 2 \mathrm{H}), 7.29(\mathrm{~m}, 3 \mathrm{H}), 5.46(\mathrm{~d}, J=0.8 \mathrm{~Hz}, 1 \mathrm{H}), 5.40(\mathrm{~d}, J=$ $0.8 \mathrm{~Hz}, 1 \mathrm{H}), 2.45(\mathrm{~m}, 2 \mathrm{H}), 2.33(\mathrm{~m}, 2 \mathrm{H}), 1.83(\mathrm{~m}, 1 \mathrm{H})$, $1.55(\mathrm{~m}, 1 \mathrm{H}), 0.01(\mathrm{~s}, 9 \mathrm{H}) \mathrm{ppm} .{ }^{13} \mathrm{C}\left\{{ }^{1} \mathrm{H}\right\}$ NMR $(101 \mathrm{MHz}$, $\left.\mathrm{CDCl}_{3}, \delta\right): 151.8,139.7,128.1,127.8,127.2,112.3,79.0,37.3$, 13.7, 1.7 ppm. HRMS (ESI): $\left[\mathrm{M}^{+}\right]$calcd. for $\mathrm{C}_{15} \mathrm{H}_{22} \mathrm{OSi}$, 246.1434; found, 246.1431. IR (film): 2988 (w), 2956 (w), $2901(w), 2895(w), 2254(w), 1252(w), 1126(w), 992(w)$, $906(\mathrm{~s}), 841(\mathrm{~m}) \mathrm{cm}^{-1}$.

Trimethyl(1-(1-(p-tolyl)vinyl)cyclobutoxy)silane (4b).<smiles>C=C(c1ccc(C)cc1)C1(OC)CCC1</smiles>

Compound $\mathbf{4 b}$ was prepared according to general method $\mathrm{D}$ using 1-(1-(p-tolyl)vinyl)cyclobutanol (3b, $0.45 \mathrm{~g}, 2.4 \mathrm{mmol}$, 1.0 equiv), $\mathrm{Et}_{3} \mathrm{~N}(0.50 \mathrm{~mL}, 3.6 \mathrm{mmol}, 1.5$ equiv), and $\mathrm{TMSCl}$ $(0.37 \mathrm{~mL}, 2.9 \mathrm{mmol}, 1.3$ equiv). The title compound $4 \mathrm{~b}$ was obtained as a colorless oil $(0.56 \mathrm{~g}, 2.1 \mathrm{mmol}, 90 \%$ yield $) . R_{f}$ (silica, pentane): $0.8 .{ }^{1} \mathrm{H} \mathrm{NMR}\left(400 \mathrm{MHz}, \mathrm{CDCl}_{3}, \delta\right): 7.44$ (dd, $J=8.2,2.1 \mathrm{~Hz}, 2 \mathrm{H}), 7.11(\mathrm{~m}, 2 \mathrm{H}), 5.44(\mathrm{~s}, 1 \mathrm{H}), 5.35(\mathrm{~s}, 1 \mathrm{H})$, $2.48-2.39(\mathrm{~m}, 2 \mathrm{H}), 2.37-2.21(\mathrm{~m}, 5 \mathrm{H}), 1.83(\mathrm{~m}, 1 \mathrm{H}), 1.53(\mathrm{~m}$, $1 \mathrm{H}), 0.00(\mathrm{~m}, 9 \mathrm{H})$ ppm. ${ }^{13} \mathrm{C}\left\{{ }^{1} \mathrm{H}\right\} \mathrm{NMR}\left(101 \mathrm{MHz}, \mathrm{CDCl}_{3}, \delta\right)$ : 151.6, 136.9, 136.6, 128.5, 128.0, 111.7, 79.1, 37.2, 21.3, 13.7, 1.8 ppm. HRMS (ESI): $\left[\mathrm{M}^{+}\right]$calcd. for $\mathrm{C}_{16} \mathrm{H}_{24} \mathrm{OSi}, 260.1591$; found, 260.1591. IR (film): 2989 (w), 2988 (w), 2970 (w), 2958 (w), $2946(\mathrm{w}), 2917(\mathrm{w}), 2365(\mathrm{~m}), 2358(\mathrm{~m}), 2343(\mathrm{~m}), 2335$ (m), $2328(\mathrm{~m}), 2254(\mathrm{w}), 1510(\mathrm{w}), 1265(\mathrm{~s}), 1253(\mathrm{~m}), 1161$ (m), $1125(\mathrm{~m}), 1118(\mathrm{~m}), 1086(\mathrm{w}), 991(\mathrm{~m}), 886(\mathrm{~m}), 876$ (m), 863 (w), $856(\mathrm{~m}), 845$ (s), $837(\mathrm{~m}), 828(\mathrm{~m}), \mathrm{cm}^{-1}$. (4c).

(1-(1-(4-(tert-Butyl)phenyl)vinyl)cyclobutoxy)trimethylsilane

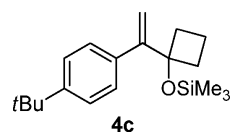

Compound $4 \mathrm{c}$ was prepared according to general method $\mathrm{D}$ using 1-(1-(4-(tert-butyl)phenyl)vinyl)cyclobutanol (3c, $0.45 \mathrm{~g}$, $2.0 \mathrm{mmol}, 1.0$ equiv), $\mathrm{Et}_{3} \mathrm{~N}(0.40 \mathrm{~mL}, 2.9 \mathrm{mmol}, 1.5$ equiv), and TMSCl $(0.30 \mathrm{~mL}, 2.3 \mathrm{mmol}, 1.3$ equiv). The title compound
$4 \mathrm{c}$ was obtained as a pale yellow oil $(0.47 \mathrm{~g}, 1.6 \mathrm{mmol}, 90 \%$ yield). $R_{f}$ (silica, pentane): 0.8. ${ }^{1} \mathrm{H}$ NMR $\left(400 \mathrm{MHz}, \mathrm{CDCl}_{3}, \delta\right)$ : $7.46(\mathrm{~m}, 2 \mathrm{H}), 7.32(\mathrm{~m}, 2 \mathrm{H}), 5.44(\mathrm{~d}, J=1.0 \mathrm{~Hz}, 1 \mathrm{H}), 5.35(\mathrm{~d}, J=$ $0.9 \mathrm{~Hz}, 1 \mathrm{H}), 2.44(\mathrm{~m}, 2 \mathrm{H}), 2.31(\mathrm{~m}, 2 \mathrm{H}), 1.82(\mathrm{~m}, 1 \mathrm{H}), 1.53(\mathrm{~m}$, $1 \mathrm{H}), 1.33$ (s, 9H), 0.00 (s, 9H) ppm. ${ }^{13} \mathrm{C}\left\{{ }^{1} \mathrm{H}\right\}$ NMR (101 MHz, $\left.\mathrm{CDCl}_{3}, \delta\right): 151.7,150.2,136.8,127.9,124.8,111.8,79.2,37.35$, 34.7, 31.6, 13.8, 1.9 ppm. HRMS (ESI): $[\mathrm{M}+\mathrm{Na}]^{+}$calcd. for $\mathrm{C}_{19} \mathrm{H}_{30} \mathrm{NaOSi}^{+}$, 325.1958; found, 325.1952. IR (film): 3091 (w), 3049 (w), $3034(\mathrm{w}), 2987(\mathrm{w}), 2959(\mathrm{~m}), 2958(\mathrm{~m}), 2903(\mathrm{w})$, $2871(\mathrm{w}), 2360(\mathrm{~m}), 1671(\mathrm{w}), 1512(\mathrm{w}), 1511(\mathrm{w}), 1364(\mathrm{w})$, 1251 (s), $1163(\mathrm{~m}), 1125(\mathrm{~m}), 1115(\mathrm{~m}), 1018(\mathrm{~m}), 992(\mathrm{~m})$, $928(\mathrm{~m}), 913(\mathrm{~m}), 899(\mathrm{w}), 881(\mathrm{~m}), 865(\mathrm{~m}), 841(\mathrm{~s}) \mathrm{cm}^{-1}$.

(1-(1-([1,1'-Biphenyl]-4-yl)vinyl)cyclobutoxy)trimethylsilane (4d).

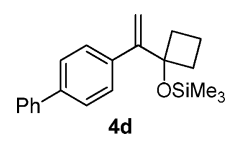

Compound 4d was prepared according to general method D using 1-(1-([1,1'-biphenyl]-4-yl)vinyl)cyclobutanol (3d, $0.48 \mathrm{~g}$, $1.9 \mathrm{mmol}, 1.0$ equiv), $\mathrm{Et}_{3} \mathrm{~N}$ (0.40 mL, $2.9 \mathrm{mmol}, 1.5$ equiv), and TMSCl ( $0.30 \mathrm{~mL}, 2.3 \mathrm{mmol}, 1.3$ equiv). The title compound $\mathbf{4 d}$ was obtained as a white solid $(0.500 \mathrm{~g}, 1.55 \mathrm{mmol}, 81 \%$ yield $)$. $R_{f}$ (silica, pentane): 0.8. mp $54{ }^{\circ} \mathrm{C} .{ }^{1} \mathrm{H}$ NMR (400 $\mathrm{MHz}, \mathrm{CDCl}_{3}$, $\delta): 7.63(\mathrm{~m}, 4 \mathrm{H}), 7.56(\mathrm{~m}, 2 \mathrm{H}), 7.45(\mathrm{~m}, 2 \mathrm{H}), 7.36(\mathrm{~m}, 1 \mathrm{H})$, $5.55(\mathrm{~d}, J=0.8 \mathrm{~Hz}, 1 \mathrm{H}), 5.43(\mathrm{~d}, J=0.8 \mathrm{~Hz}, 1 \mathrm{H}), 2.49(\mathrm{~m}, 2 \mathrm{H})$, $2.37(\mathrm{~m}, 2 \mathrm{H}), 1.86(\mathrm{~m}, 1 \mathrm{H}), 1.59(\mathrm{~m}, 1 \mathrm{H}), 0.04(\mathrm{~s}, 9 \mathrm{H}) \mathrm{ppm}$. ${ }^{13} \mathrm{C}\left\{{ }^{1} \mathrm{H}\right\}$ NMR (101 MHz, $\left.\mathrm{CDCl}_{3}, \delta\right): 151.4,141.0,139.9,138.5$, 128.9, 128.5, 127.3, 127.1, 126.5, 112.3, 79.1, 37.3, 13.7, 1.8 ppm. HRMS (ESI): $[\mathrm{M}+\mathrm{Na}]^{+}$calcd. for $\mathrm{C}_{21} \mathrm{H}_{26} \mathrm{NaOSi}^{+}, 345.1645$; found, 345.1639. IR (film): 3096 (w), 3090 (w), 3080 (w), 3063 $(\mathrm{w}), 3050(\mathrm{w}), 3032(\mathrm{w}), 2988(\mathrm{w}), 2988(\mathrm{w}), 2955(\mathrm{w})$, $2917(\mathrm{w}), 2901(\mathrm{w}), 2900(\mathrm{w}), 2895(\mathrm{w}), 2874(\mathrm{w}), 2330(\mathrm{w})$, $2253(\mathrm{w}), 1489(\mathrm{w}), 1251(\mathrm{~m}), 1161(\mathrm{w}), 1125(\mathrm{w}), 1089(\mathrm{w})$, $990(\mathrm{~m}), 906(\mathrm{~s}), 842(\mathrm{~m}) \mathrm{cm}^{-1}$.

(1-(1-(4-Methoxyphenyl)vinyl)cyclobutoxy)trimethylsilane (4e).

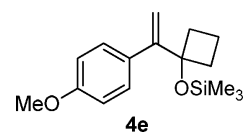

Compound 4e was prepared according to general method D using 1-(1-(4-methoxyphenyl)vinyl)cyclobutanol (3e, $0.50 \mathrm{~g}$, $2.5 \mathrm{mmol}, 1.0$ equiv), $\mathrm{Et}_{3} \mathrm{~N}(0.51 \mathrm{~mL}, 3.7 \mathrm{mmol}, 1.5$ equiv), and TMSCl $(0.370 \mathrm{~mL}, 2.93 \mathrm{mmol}, 1.30$ equiv). The title compound $4 \mathrm{e}$ was obtained as a colorless oil $(0.55 \mathrm{~g}, 2.0 \mathrm{mmol}, 81 \%$ yield). $R_{f}$ (silica, pentane): 0.7. ${ }^{1} \mathrm{H}$ NMR $\left(400 \mathrm{MHz}, \mathrm{CDCl}_{3}, \delta\right)$ : $7.49(\mathrm{~m}, 2 \mathrm{H}), 6.83(\mathrm{~m}, 2 \mathrm{H}), 5.40(\mathrm{~s}, 1 \mathrm{H}), 5.30(\mathrm{~d}, J=0.8 \mathrm{~Hz}$, $1 \mathrm{H}), 3.81(\mathrm{~s}, 3 \mathrm{H}), 2.43(\mathrm{~m}, 2 \mathrm{H}), 2.31(\mathrm{~m}, 2 \mathrm{H}), 1.82(\mathrm{~m}, 1 \mathrm{H})$, $1.53(\mathrm{~m}, 1 \mathrm{H}), 0.00(\mathrm{~d}, J=1.1 \mathrm{~Hz}, 9 \mathrm{H}) \mathrm{ppm} .{ }^{13} \mathrm{C}\left\{{ }^{1} \mathrm{H}\right\} \mathrm{NMR}$ $\left(101 \mathrm{MHz}, \mathrm{CDCl}_{3}, \delta\right): 159.0,151.0,132.0,129.2,113.2,111.0$, 79.2, 55.4, 37.3, 13.8, 1.8 ppm. HRMS (ESI): $[\mathrm{M}+\mathrm{Na}]^{+}$calcd. for $\mathrm{C}_{16} \mathrm{H}_{24} \mathrm{NaO}_{2} \mathrm{Si}^{+}$, 299.1438; found, 299.1435. IR (film): $3097(w), 2988(w), 2955(w), 2903(w), 2837(w), 2254(w)$, $1609(\mathrm{w}), 1511(\mathrm{~m}), 1250(\mathrm{~m}), 1182(\mathrm{~m}), 1161(\mathrm{~m})$, $1124(\mathrm{~m}), 991(\mathrm{~m}), 912(\mathrm{~s}), 908$ (s), $838(\mathrm{~m}) \mathrm{cm}^{-1}$.

(1-(1-(4-Fluorophenyl)vinyl)cyclobutoxy)trimethylsilane (4f).

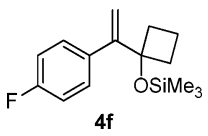


Compound $4 \mathrm{f}$ was prepared according to general method $\mathrm{D}$ using 1-(1-(4-fluorophenyl)vinyl)cyclobutanol (3f, $0.50 \mathrm{~g}$, $2.6 \mathrm{mmol}, 1.0$ equiv), $\mathrm{Et}_{3} \mathrm{~N}$ ( $0.54 \mathrm{~mL}, 3.9 \mathrm{mmol}, 1.5$ equiv), and TMSCl $(0.40 \mathrm{~mL}, 3.1 \mathrm{mmol}, 1.3$ equiv). The title compound 4f was obtained as a colorless oil (0.64 g, $2.4 \mathrm{mmol}$, 90\% yield). $R_{f}$ (silica, pentane): 0.6. ${ }^{1} \mathrm{H}$ NMR (400 $\left.\mathrm{MHz}, \mathrm{CDCl}_{3}, \delta\right): 7.51$ $(\mathrm{m}, 2 \mathrm{H}), 6.98(\mathrm{~m}, 2 \mathrm{H}), 5.41(\mathrm{~d}, J=0.7 \mathrm{~Hz}, 1 \mathrm{H}), 5.37(\mathrm{~s}, 1 \mathrm{H})$, $2.41\left(\mathrm{~m}, 2 \mathrm{H}, \mathrm{CH}_{2}\right), 2.32(\mathrm{~m}, 2 \mathrm{H}), 1.98(\mathrm{~m}, 1 \mathrm{H}), 1.53(\mathrm{~m}, 1 \mathrm{H})$, $0.01(\mathrm{~m}, 9 \mathrm{H})$ ppm. ${ }^{13} \mathrm{C}\left\{{ }^{1} \mathrm{H}\right\} \mathrm{NMR}\left(101 \mathrm{MHz} \mathrm{CDCl}_{3}, \delta\right): 162.3$ $(\mathrm{d}, J=246.0 \mathrm{~Hz}), 150.8,135.5(\mathrm{~d}, J=3.4 \mathrm{~Hz}), 129.7(\mathrm{~d}, J=$ $7.7 \mathrm{~Hz}), 114.6$ (d, $J=21.0 \mathrm{~Hz}), 112.1,79.0,37.2,13.7,1.7$ ppm. ${ }^{19} \mathrm{~F}\left\{{ }^{1} \mathrm{H}\right\}$ NMR $\left(376 \mathrm{MHz}, \mathrm{CDCl}_{3}, \delta\right.$ ): -115.74 ppm. HRMS (ESI): $\left[\mathrm{M}^{+}\right]$calcd. for $\mathrm{C}_{15} \mathrm{H}_{21} \mathrm{FOSi}$, 264.1340; found, 264.1339. IR (film): 4014 (s), 3154 (w), 3142 (w), $3122(\mathrm{w}), 2988$ (w), $2966(w), 2956(w), 2901(w), 2254(w), 1604(w), 1509(w)$, $1251(\mathrm{w}), 1162(\mathrm{w}), 990(\mathrm{w}), 906(\mathrm{~m}), 841(\mathrm{~m}) \mathrm{cm}^{-1}$.

(1-(1-(4-Chlorophenyl)vinyl)cyclobutoxy)trimethylsilane (4g).

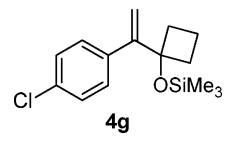

Compound $\mathbf{4 g}$ was prepared according to general method $\mathrm{D}$ using 1-(1-(4-chlorophenyl)vinyl)cyclobutanol $(3 \mathrm{~g}, 0.40 \mathrm{~g}$, $1.9 \mathrm{mmol}, 1.0$ equiv), $\mathrm{Et}_{3} \mathrm{~N}(0.40 \mathrm{~mL}, 2.9 \mathrm{mmol}, 1.5$ equiv), and TMSCl $(0.30 \mathrm{~mL}, 2.3 \mathrm{mmol}, 1.3$ equiv). The title compound $4 \mathrm{~g}$ was obtained as a colorless oil $(0.43 \mathrm{~g}, 1.5 \mathrm{mmol}, 80 \%$ yield $)$. $R_{f}$ (silica, pentane): 0.7. ${ }^{1} \mathrm{H} \mathrm{NMR}\left(400 \mathrm{MHz}, \mathrm{CDCl}_{3}, \delta\right): 7.48(\mathrm{~m}$, $2 \mathrm{H}), 7.26(\mathrm{~m}, 2 \mathrm{H}), 5.45(\mathrm{~d}, J=0.6 \mathrm{~Hz}, 1 \mathrm{H}), 5.39(\mathrm{~d}, J=$ $0.6 \mathrm{~Hz}, 1 \mathrm{H}), 2.45-2.21(\mathrm{~m}, 4 \mathrm{H}), 1.82(\mathrm{~m}, 1 \mathrm{H}), 1.53(\mathrm{~m}, 1 \mathrm{H})$, $0.00(\mathrm{~s}, 9 \mathrm{H})$ ppm. ${ }^{13} \mathrm{C}\left\{{ }^{1} \mathrm{H}\right\} \mathrm{NMR}\left(101 \mathrm{MHz}, \mathrm{CDCl}_{3}, \delta\right): 150.7$, 137.9, 133.1, 129.4, 128.0, 112.7, 78.8, 37.1, 13.7, 1.8 ppm. HRMS (ESI): $\left[\mathrm{M}^{+}\right]$calcd. for $\mathrm{C}_{15} \mathrm{H}_{21} \mathrm{ClOSi}, 280.1045$; found, 280.1045 . IR (film): 3098 (w), 2988 (w), 2956 (w), 2902 (w), 2901 (w), $2894(\mathrm{w}), 2254(\mathrm{w}), 1491(\mathrm{~m}), 1252(\mathrm{~m}), 1161(\mathrm{~m}), 1125(\mathrm{~m})$, 1096 (m), 1015 (m), 991 (m), 905 (s), 860 (m), $839(\mathrm{~m}) \mathrm{cm}^{-1}$.

Trimethyl(1-(1-(m-tolyl)vinyl)cyclobutoxy)silane (4h).

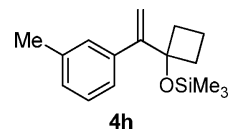

Compound $4 \mathrm{~h}$ was prepared according to the general method D using 1-(1-(m-tolyl)vinyl)cyclobutanol (3h, $0.68 \mathrm{~g}$, $3.6 \mathrm{mmol}, 1.0$ equiv), $\mathrm{Et}_{3} \mathrm{~N}$ ( $0.75 \mathrm{~mL}, 5.4 \mathrm{mmol}, 1.5$ equiv), and TMSCl (0.55 mL, $4.3 \mathrm{mmol}, 1.3$ equiv). The title compound $4 \mathrm{~h}$ was obtained as a colorless oil $(0.76 \mathrm{~g}, 2.9 \mathrm{mmol}$, $81 \%$ yield). $R_{f}$ (silica, pentane): $0.8 .{ }^{1} \mathrm{H}$ NMR $(400 \mathrm{MHz}$, $\left.\mathrm{CDCl}_{3}, \delta\right): 7.35(\mathrm{~m}, 2 \mathrm{H}), 7.19(\mathrm{~m}, 1 \mathrm{H}), 7.08(\mathrm{~m}, 1 \mathrm{H}), 5.42$ $(\mathrm{m}, 1 \mathrm{H}), 5.37(\mathrm{~m}, 1 \mathrm{H}), 2.43(\mathrm{~m}, 2 \mathrm{H}), 2.34(\mathrm{~m}, 5 \mathrm{H}), 1.81$ $(\mathrm{m}, 1 \mathrm{H}), 1.53(\mathrm{~m}, 1 \mathrm{H}), 0.01(\mathrm{~m}, 9 \mathrm{H}) \mathrm{ppm} .{ }^{13} \mathrm{C}\left\{{ }^{1} \mathrm{H}\right\} \mathrm{NMR}$ $\left(101 \mathrm{MHz}, \mathrm{CDCl}_{3}, \delta\right): 152.0,139.7,137.2,128.8,128.0,127.7$, 125.3, 112.3, 79.0, 37.2, 21.7, 13.7, 1.8 ppm. HRMS (ESI): $[\mathrm{M}+\mathrm{Na}]^{+}$calcd. for $\mathrm{C}_{16} \mathrm{H}_{24} \mathrm{NaOSi}^{+}, 283.1489$; found, 283.1485. IR (film): 3095 (w), 3092 (w), 3041 (w), 3034 (w), 3026 (w), $2988(\mathrm{w}), 2954(\mathrm{w}), 2953(\mathrm{w}), 2901(\mathrm{w}), 2874(\mathrm{w}), 1250(\mathrm{~s})$, 1158 (m), 1124 (m), 991 (s), 913 (m), 840 (s) $\mathrm{cm}^{-1}$. (4i).

(1-(1-(3-Methoxyphenyl)vinyl)cyclobutoxy)trimethylsilane

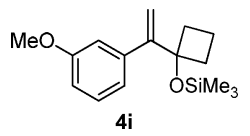

Compound $4 \mathbf{i}$ was prepared according to general method D using 1-(1-(3-methoxyphenyl)vinyl)cyclobutanol (3i, $0.15 \mathrm{~g}$, $0.73 \mathrm{mmol}, 1.0$ equiv), $\mathrm{Et}_{3} \mathrm{~N}(0.15 \mathrm{~mL}, 1.1 \mathrm{mmol}, 1.5$ equiv), and TMSCl $(0.11 \mathrm{~mL}, 0.88 \mathrm{mmol}, 1.3$ equiv). The title compound $4 \mathbf{i}$ was obtained as a colorless oil (0.15 g, $0.54 \mathrm{mmol}, 74 \%$ yield). $R_{f}$ (silica, pentane): 0.6. ${ }^{1} \mathrm{H}$ NMR $\left(400 \mathrm{MHz}, \mathrm{CDCl}_{3}, \delta\right): 7.22(\mathrm{~m}, 1 \mathrm{H}), 7.13(\mathrm{~m}, 2 \mathrm{H}), 6.82$ (ddd, $J=8.1,2.6,1.1 \mathrm{~Hz}, 1 \mathrm{H}), 5.46(\mathrm{~d}, J=0.9 \mathrm{~Hz}, 1 \mathrm{H}), 5.39$ $(\mathrm{d}, J=0.8 \mathrm{~Hz}, 1 \mathrm{H}), 3.81(\mathrm{~s}, 3 \mathrm{H}), 2.43(\mathrm{~m}, 2 \mathrm{H}), 2.31$ $(\mathrm{m}, 2 \mathrm{H}), 1.82(\mathrm{~m}, 1 \mathrm{H}), 1.54(\mathrm{~m}, 1 \mathrm{H}), 0.04(\mathrm{~s}, 9 \mathrm{H}) \mathrm{ppm}$. ${ }^{13} \mathrm{C}\left\{{ }^{1} \mathrm{H}\right\}$ NMR $\left(101 \mathrm{MHz}, \mathrm{CDCl}_{3}\right.$; one $\mathrm{C}(\mathrm{sp} 2)$ signal was not resolved, $\delta): 159.2,151.8,141.3,128.8,120.8,114.0,112.8$, 79.1, 55.4, 37.2, 13.8, 1.9 ppm. HRMS (ESI): $\left[\mathrm{M}^{+}\right]$calcd. for $\mathrm{C}_{16} \mathrm{H}_{24} \mathrm{O}_{2} \mathrm{Si}, 276.1540$; found, 276.1541. IR (film): $3080(\mathrm{w})$, 2988 (w), $2955(\mathrm{w}), 2920(\mathrm{w}), 2905(\mathrm{w}), 2896(\mathrm{w}), 2875$ $(\mathrm{w}), 2832(\mathrm{w}), 1598(\mathrm{~m}), 1578(\mathrm{~m}), 1489(\mathrm{~m}), 1287(\mathrm{~m})$, 1251 (s), $1158(\mathrm{~m}), 1125$ (m), $992(\mathrm{~s}), 915(\mathrm{~m}), 914(\mathrm{~m})$, $841(\mathrm{~s}) \mathrm{cm}^{-1}$.

(1-(1-(3-Fluorophenyl)vinyl)cyclobutoxy)trimethylsilane (4j).

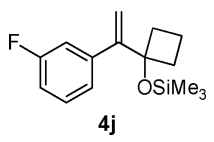

Compound $4 \mathbf{j}$ was prepared according to general method $\mathrm{D}$ using 1-(1-(3-fluorophenyl)vinyl)cyclobutanol (3j, $0.67 \mathrm{~g}$, $3.5 \mathrm{mmol}, 1.0$ equiv), $\mathrm{Et}_{3} \mathrm{~N}$ (0.73 mL, $5.2 \mathrm{mmol}, 1.5$ equiv), and TMSCl $(0.53 \mathrm{~mL}, 4.2 \mathrm{mmol}, 1.3$ equiv). The title compound $4 \mathbf{j}$ was obtained as a colorless oil $(0.74 \mathrm{~g}, 2.8 \mathrm{mmol}$, $80 \%$ yield). $R_{f}$ (silica, pentane): 0.7. ${ }^{1} \mathrm{H}$ NMR (400 MHz, $\left.\mathrm{CDCl}_{3}, \delta\right): 7.31(\mathrm{~m}, 3 \mathrm{H}), 6.99(\mathrm{tdd}, J=8.3,2.6,1.2 \mathrm{~Hz}, 1 \mathrm{H})$, $5.52(\mathrm{~d}, J=0.6 \mathrm{~Hz}, 1 \mathrm{H}), 5.46(\mathrm{~s}, 1 \mathrm{H}), 2.52-2.23(\mathrm{~m}, 4 \mathrm{H})$, $1.87(\mathrm{~m}, 1 \mathrm{H}), 1.58(\mathrm{~m}, 1 \mathrm{H}), 0.05(\mathrm{~s}, 9 \mathrm{H}) \mathrm{ppm} .{ }^{13} \mathrm{C}\left\{{ }^{1} \mathrm{H}\right\}$ NMR $\left(101 \mathrm{MHz}, \mathrm{CDCl}_{3}, \delta\right): 162.5(\mathrm{~d}, J=243.9 \mathrm{~Hz}), 150.8$ $(\mathrm{d}, J=2.2 \mathrm{~Hz}), 141.9(\mathrm{~d}, J=7.7 \mathrm{~Hz}), 129.2(\mathrm{~d}, J=8.3 \mathrm{~Hz})$, $123.7(\mathrm{~d}, J=2.8 \mathrm{~Hz}), 115.0(\mathrm{~d}, J=22.3 \mathrm{~Hz}), 114.0(\mathrm{~d}, J=$ $21.1 \mathrm{~Hz}$ ), 113.2, 78.8, 37.1, 13.6, 1.7 ppm. HRMS (ESI): $\left[\mathrm{M}^{+}\right]$calcd. for $\mathrm{C}_{15} \mathrm{H}_{21}$ FOSi, 264.1340; found, 264.1339. IR (film): $3091(\mathrm{w}), 2988(\mathrm{w}), 2956(\mathrm{w}), 2916(\mathrm{w}), 2901(\mathrm{w})$, $2875(\mathrm{w}), 2874(\mathrm{w}), 2360(\mathrm{~m}), 2342(\mathrm{~m}), 1614(\mathrm{w})$, $1580(\mathrm{~m}), 1488(\mathrm{~m}), 1251(\mathrm{~s}), 1155(\mathrm{~m}), 1121(\mathrm{~m}), 990(\mathrm{~s})$, $917(\mathrm{~s}), 840(\mathrm{~s}) \mathrm{cm}^{-1}$.

Trimethyl(1-(1-(o-tolyl)vinyl)cyclobutoxy)silane (4k).

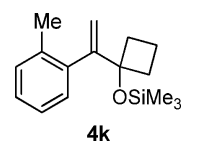

Compound $4 \mathbf{k}$ was prepared according to the general method D using 3k (0.340 g, $1.79 \mathrm{mmol}, 1.00$ equiv), $\mathrm{Et}_{3} \mathrm{~N}$ (0.37 mL, $2.7 \mathrm{mmol}, 1.5$ equiv), and $\mathrm{TMSCl}(0.27 \mathrm{~mL}$, $2.1 \mathrm{mmol}, 1.3$ equiv). The title compound $4 \mathbf{k}$ was obtained as a colorless oil $\left(317 \mathrm{mg}, 2.68 \mathrm{mmol}, 68 \%\right.$ yield). $R_{f}$ (silica, pentane:EtOAc 99:1): 0.8. ${ }^{1} \mathrm{H}$ NMR $\left(400 \mathrm{MHz}, \mathrm{CDCl}_{3}, \delta\right)$ : $7.25-7.09(\mathrm{~m}, 4 \mathrm{H}), 5.55(\mathrm{~d}, J=1.6 \mathrm{~Hz}, 1 \mathrm{H}), 4.98(\mathrm{~d}, J=$ $1.6 \mathrm{~Hz}, 1 \mathrm{H}), 2.46-2.35(\mathrm{~m}, 2 \mathrm{H}), 2.31$ (s, 3H), 2.28-2.18 $(\mathrm{m}, 2 \mathrm{H}), 1.84-1.71(\mathrm{~m}, 1 \mathrm{H}), 1.53-1.38(\mathrm{~m}, 1 \mathrm{H}), 0.10$ (s, 9H) ppm. ${ }^{13} \mathrm{C}\left\{{ }^{1} \mathrm{H}\right\}$ NMR $\left(101 \mathrm{MHz}, \mathrm{CDCl}_{3}, \delta\right): 151.7$, 140.6, 136.2, 129.8, 129.5, 126.7, 124.7, 113.6, 79.5, 36.1, 20.5, 13.6, 1.9 ppm. HRMS (ESI): $\left[\mathrm{M}^{+}\right]$calcd for $\mathrm{C}_{16} \mathrm{H}_{24} \mathrm{OSi}$, 260.1596; found, 260.1586. IR (film): 2987 (m), 2958 (m), $1489(\mathrm{w}), 1381(\mathrm{w}), 1250(\mathrm{~s}), 1167(\mathrm{w}), 1079(\mathrm{w}), 995(\mathrm{~m})$, $926(\mathrm{~m}), 841(\mathrm{~s}), 760(\mathrm{~m}), 732(\mathrm{~m}) \mathrm{cm}^{-1}$. 

(4I).

(1-(1-(3,4-Dimethylphenyl)vinyl)cyclobutoxy)trimethylsilane

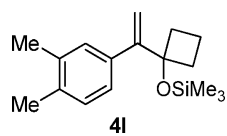

Compound $\mathbf{4 1}$ was prepared according to the general method D using 1-(3-phenylprop-1-en-2-yl)cyclobutanol (31, $0.680 \mathrm{~g}$, $3.61 \mathrm{mmol}, 1.00$ equiv), $\mathrm{Et}_{3} \mathrm{~N}$ ( $0.75 \mathrm{~mL}, 5.4 \mathrm{mmol}, 1.5$ equiv), and TMSCl $(0.55 \mathrm{~mL}, 4.3 \mathrm{mmol}, 1.3$ equiv). The title compound 41 was obtained as a colorless oil $(0.610 \mathrm{~g}, 2.34 \mathrm{mmol}$, $65 \%$ yield). $R_{f}$ (silica, pentane:EtOAc 99:1): 0.9. ${ }^{1} \mathrm{H}$ NMR $\left(400 \mathrm{MHz}, \mathrm{CDCl}_{3}, \delta\right): 7.32(\mathrm{~d}, J=2.0 \mathrm{~Hz}, 1 \mathrm{H}), 7.29(\mathrm{dd}, J=$ $7.8,2.0 \mathrm{~Hz}, 1 \mathrm{H}), 7.07$ (d, $J=7.8 \mathrm{~Hz}, 1 \mathrm{H}), 5.42(\mathrm{~d}, J=1.0 \mathrm{~Hz}$, $1 \mathrm{H}), 5.34(\mathrm{~d}, J=1.0 \mathrm{~Hz}, 1 \mathrm{H}), 2.49-2.38(\mathrm{~m}, 2 \mathrm{H}), 2.36-2.29$ $(\mathrm{m}, 2 \mathrm{H}), 2.28(\mathrm{~s}, 3 \mathrm{H}), 2.27(\mathrm{~s}, 3 \mathrm{H}), 1.90-1.76(\mathrm{~m}, 1 \mathrm{H})$, 1.64-1.44 (m, 1H), $0.04(\mathrm{~s}, 9 \mathrm{H})$ ppm. ${ }^{13} \mathrm{C}\left\{{ }^{1} \mathrm{H}\right\}$ NMR (101 $\left.\mathrm{MHz}, \mathrm{CDCl}_{3}, \delta\right): 151.6,137.0,135.6,135.4,129.1,128.9,125.5$, 111.6, 79.0, 37.0, 19.9, 19.4, 13.6, 1.7 ppm. HRMS (ESI): [ $\left.\mathrm{M}^{+}\right]$ calcd for $\mathrm{C}_{17} \mathrm{H}_{26} \mathrm{OSi}$, 274.1747; found, 274.1751. IR (film): $2969(\mathrm{~m}), 2901(\mathrm{~m}), 2361(\mathrm{w}), 1505(\mathrm{w}), 1451(\mathrm{w}), 1407(\mathrm{w})$, $1250(\mathrm{~s}), 1118(\mathrm{~m}), 991(\mathrm{~m}), 839(\mathrm{~s}), 753(\mathrm{~m}) \mathrm{cm}^{-1}$.

(1-(1-(3,4-Dimethoxyphenyl)vinyl)cyclobutoxy)trimethylsilane $(4 \mathrm{~m})$.

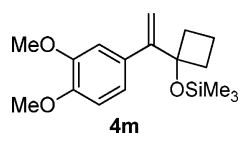

Compound $4 \mathrm{~m}$ was prepared according to general method $\mathrm{D}$ using 1-(1-(3,4-dimethoxyphenyl)vinyl)cyclobutanol (3m, $0.20 \mathrm{~g}, 0.85 \mathrm{mmol}, 1.0$ equiv), $\mathrm{Et}_{3} \mathrm{~N}(0.18 \mathrm{~mL}, 1.3 \mathrm{mmol}$, 1.5 equiv), and TMSCl ( $0.13 \mathrm{~mL}, 1.0 \mathrm{mmol}, 1.3$ equiv). The title compound $4 \mathrm{~m}$ was obtained as a colorless oil $(0.13 \mathrm{~g}$, $0.42 \mathrm{mmol}, 50 \%$ yield). $R_{f}$ (silica, pentane): $0.4 .{ }^{1} \mathrm{H}$ NMR $\left(400 \mathrm{MHz}, \mathrm{CDCl}_{3}, \delta\right): 7.17(\mathrm{~d}, J=2.1 \mathrm{~Hz}, 1 \mathrm{H}), 7.11(\mathrm{dd}, J=$ 8.4, $2.1 \mathrm{~Hz}, 1 \mathrm{H}), 6.80(\mathrm{~d}, J=8.4 \mathrm{~Hz}, 1 \mathrm{H}), 5.42(\mathrm{~d}, J=0.8 \mathrm{~Hz}$, $1 \mathrm{H}), 5.31(\mathrm{~d}, J=0.8 \mathrm{~Hz}, 1 \mathrm{H}), 3.88(\mathrm{~s}, 3 \mathrm{H}), 3.88(\mathrm{~s}, 3 \mathrm{H}), 2.40$ $(\mathrm{m}, 2 \mathrm{H}), 2.29(\mathrm{~m}, 2 \mathrm{H}), 1.82(\mathrm{~m}, 1 \mathrm{H}), 1.52(\mathrm{~m}, 1 \mathrm{H}), 0.02$ (s, 9H) ppm. ${ }^{13} \mathrm{C}\left\{{ }^{1} \mathrm{H}\right\}$ NMR (101 MHz, $\mathrm{CDCl}_{3}, \delta$ ): 151.0, $148.4,148.1,132.3,120.5,111.6,111.3,110.5,79.2,55.9,37.1$, $13.7,1.8 \mathrm{ppm}$. One aliphatic signal was not resolved. HRMS (ESI): $\left[\mathrm{M}^{+}\right]$calcd. for $\mathrm{C}_{17} \mathrm{H}_{26} \mathrm{O}_{3} \mathrm{Si}$, 306.1646; found, 306.1645. IR (film): 2988 (w), 2988 (w), 2955 (w), 2907 (w), 2873 (w), $2835(\mathrm{w}), 2255(\mathrm{w}), 1516(\mathrm{~s}), 1272(\mathrm{~m}), 1259(\mathrm{~s}), 1250(\mathrm{~s})$, $1144(\mathrm{~m}), 1123(\mathrm{~m}), 1122(\mathrm{~m}), 1029(\mathrm{~m}), 992(\mathrm{~m}), 991(\mathrm{~m})$, $912(\mathrm{~s}), 842(\mathrm{~s}) \mathrm{cm}^{-1}$.

(1-(1-(Benzo[d][1,3]dioxol-5-yl)vinyl)cyclobutoxy)trimethylsilane $(4 \mathrm{n})$.

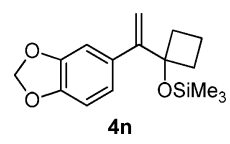

Compound $4 \mathrm{n}$ was prepared according to general method $\mathrm{D}$ using 1-(1-(benzo[d][1,3] dioxol-5-yl)vinyl)cyclobutanol (3n, $0.55 \mathrm{~g}, 2.5 \mathrm{mmol}$, 1.0 equiv), $\mathrm{Et}_{3} \mathrm{~N}$ (0.53 mL, $3.8 \mathrm{mmol}$, 1.5 equiv), and TMSCl ( $0.39 \mathrm{~mL}, 3.0 \mathrm{mmol}, 1.3$ equiv). The title compound $4 \mathrm{n}$ was obtained as a colorless oil $(0.610 \mathrm{~g}$, $2.10 \mathrm{mmol}, 83 \%$ yield). $R_{f}$ (silica, pentane): $0.4 .{ }^{1} \mathrm{H}$ NMR $\left(400 \mathrm{MHz}, \mathrm{CDCl}_{3}, \delta\right): 7.07$ (d, $\left.J=1.8 \mathrm{~Hz}, 1 \mathrm{H}\right), 7.03(\mathrm{dd}, J=$ 8.1, $1.8 \mathrm{~Hz}, 1 \mathrm{H}), 6.75(\mathrm{~d}, J=8.1 \mathrm{~Hz}, 1 \mathrm{H}), 5.94(\mathrm{~s}, 2 \mathrm{H}), 5.37$ $(\mathrm{d}, J=0.7 \mathrm{~Hz}, 1 \mathrm{H}), 5.31(\mathrm{~d}, J=0.7 \mathrm{~Hz}, 1 \mathrm{H}), 2.40(\mathrm{~m}, 2 \mathrm{H})$, $2.29(\mathrm{~m}, 2 \mathrm{H}), 1.81(\mathrm{~m}, 1 \mathrm{H}), 1.51(\mathrm{~m}, 1 \mathrm{H}), 0.02(\mathrm{~s}, 9 \mathrm{H}) \mathrm{ppm}$.
${ }^{13} \mathrm{C}\left\{{ }^{1} \mathrm{H}\right\}$ NMR $\left(101 \mathrm{MHz}, \mathrm{CDCl}_{3}, \delta\right): 151.2,147.1,146.8$ 133.7, 121.7, 111.7, 108.6, 107.7, 101.0, 79.0, 37.2, 13.7, 1.8 ppm. HRMS (ESI): $\left[\mathrm{M}^{+}\right]$calcd. for $\mathrm{C}_{16} \mathrm{H}_{22} \mathrm{O}_{3}, \mathrm{Si}$ 290.1333; found, 290.1334. IR (film): 3097 (w), 3090 (w), 3081 (w), 3080 (w), $3068(\mathrm{w}), 2988(\mathrm{w}), 2954(\mathrm{~m}), 2893(\mathrm{w}), 2774(\mathrm{w}), 2332$ (w), $1506(\mathrm{~m}), 1490(\mathrm{~s}), 1437(\mathrm{~m}), 1250$ (s), $1239(\mathrm{~s}), 1121$ (s), 1043 (s), 991 (s), 941 (m), $915(\mathrm{~m}), 854$ (s), 842 (s), 818 (m) $\mathrm{cm}^{-1}$.

Trimethyl(1-(1-(naphthalen-2-yl)vinyl)cyclobutoxy)silane (40).

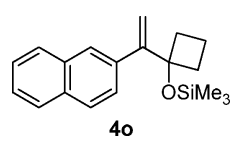

Compound 4o was prepared according to general method D using 1-(1-(naphthalen-2-yl)vinyl)cyclobutanol (3o, $0.50 \mathrm{~g}$, $2.2 \mathrm{mmol}, 1.0$ equiv), $\mathrm{Et}_{3} \mathrm{~N}(0.47 \mathrm{~mL}, 3.3 \mathrm{mmol}, 1.5$ equiv), and TMSCl $(0.34 \mathrm{~mL}, 2.7 \mathrm{mmol}, 1.3$ equiv). The title compound 4o was obtained as a colorless oil $(0.43 \mathrm{~g}, 1.4 \mathrm{mmol}$, $65 \%$ yield). $R_{f}$ (silica, pentane): $0.8 .{ }^{1} \mathrm{H}$ NMR (400 MHz, $\left.\mathrm{CDCl}_{3}, \delta\right): 8.03(\mathrm{~s}, 1 \mathrm{H}), 7.84(\mathrm{~m}, 2 \mathrm{H}), 7.78(\mathrm{~d}, J=8.6 \mathrm{~Hz}$, $1 \mathrm{H}), 7.70(\mathrm{dd}, J=8.7,1.6 \mathrm{~Hz}, 1 \mathrm{H}), 7.50-7.42(\mathrm{~m}, 2 \mathrm{H}), 5.62$ $(\mathrm{d}, J=0.9 \mathrm{~Hz}, 1 \mathrm{H}), 5.50(\mathrm{~s}, 1 \mathrm{H}), 2.51(\mathrm{~m}, 2 \mathrm{H}), 2.40(\mathrm{~m}, 2 \mathrm{H})$, $1.87(\mathrm{~m}, 1 \mathrm{H}), 1.57(\mathrm{~m}, 1 \mathrm{H}), 0.03(\mathrm{~s}, 9 \mathrm{H}) \mathrm{ppm} .{ }^{13} \mathrm{C}\left\{{ }^{1} \mathrm{H}\right\} \mathrm{NMR}$ $\left(101 \mathrm{MHz}, \mathrm{CDCl}_{3}, \delta\right): 151.7,137.0,133.3,132.7,128.4,127.5$, $127.1,127.0,126.4,125.9,125.8,112.9,79.1,37.3,13.7$, $1.8 \mathrm{ppm}$. HRMS (ESI): $\left[\mathrm{M}^{+}\right]$calcd. for $\mathrm{C}_{19} \mathrm{H}_{24} \mathrm{OSi}$, 296.1591; found, 296.1594. IR (film): 3058 (w), 2988 (w), 2954 (w), $2898(\mathrm{w}), 2873(\mathrm{w}), 1250(\mathrm{~s}), 1119(\mathrm{~m}), 990$ (s), 912 (s), $893(\mathrm{~m}), 840(\mathrm{~s}), 821(\mathrm{~m}) \mathrm{cm}^{-1}$.

Trimethyl(1-(1-(naphthalen-1-yl)vinyl)cyclobutoxy)silane (4p).

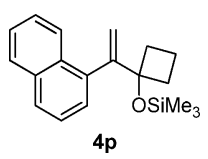

Compound $4 \mathrm{p}$ was prepared according to the general method D using 3p (1.00 g, $4.46 \mathrm{mmol}, 1.00$ equiv), $\mathrm{Et}_{3} \mathrm{~N}$ (0.93 mL, $6.7 \mathrm{mmol}, 1.5$ equiv), and TMSCl $(0.67 \mathrm{~mL}, 5.3 \mathrm{mmol}$, 1.3 equiv). The title compound $4 \mathrm{p}$ was obtained as a colorless oil (1.10 g, $3.71 \mathrm{mmol}, 83 \%$ yield). $R_{f}$ (silica, pentane:EtOAc 99:1): 0.6. ${ }^{1} \mathrm{H}$ NMR (400 $\left.\mathrm{MHz}, \mathrm{CDCl}_{3}, \delta\right): 8.15-8.06$ (m, 1H), 7.90-7.82 (m, 1H), 7.82-7.76 (m, 1H), 7.51-7.41 $(\mathrm{m}, 4 \mathrm{H}), 5.80(\mathrm{~d}, J=1.6 \mathrm{~Hz}, 1 \mathrm{H}), 5.19(\mathrm{~d}, J=1.7 \mathrm{~Hz}, 1 \mathrm{H})$, $2.58-2.41(\mathrm{~m}, 2 \mathrm{H}), 2.34-2.19(\mathrm{~m}, 2 \mathrm{H}), 1.82-1.70(\mathrm{~m}, 1 \mathrm{H})$, 1.52-1.37 (m, 1H), 0.07 (s, 9H) ppm. ${ }^{13} \mathrm{C}\left\{{ }^{1} \mathrm{H}\right\}$ NMR (101 MHz, $\left.\mathrm{CDCl}_{3}, \delta\right): 150.3,138.7,133.5,132.9,128.0,127.2,126.8,126.5$, 125.4, 125.4, 124.7, 115.2, 79.4, 36.1, 13.5, 1.9 ppm. HRMS (ESI): $\left[\mathrm{M}^{+}\right]$calcd for $\mathrm{C}_{16} \mathrm{H}_{21} \mathrm{~F}_{3} \mathrm{OSi}, 314.1308$; found, 314.1314 . IR (film): 2991 (w), $2954(\mathrm{w}), 1250(\mathrm{~m}), 1162(\mathrm{w}), 1135(\mathrm{w})$, $1115(\mathrm{w}), 992(\mathrm{~m}), 908(\mathrm{~m}), 839(\mathrm{~s}), 778(\mathrm{~s}), 732(\mathrm{~s}) \mathrm{cm}^{-1}$.

Trimethyl((3-(1-phenylvinyl)oxetan-3-yl)oxy)silane (4q).

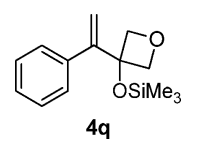

Compound $\mathbf{4 q}$ was prepared according to the general procedure, 3-(1-phenylvinyl)oxetan-3-ol (3q, $0.400 \mathrm{~g}, 2.27 \mathrm{mmol}$, 1.00 equiv), $\mathrm{Et}_{3} \mathrm{~N}$ (0.48 mL, $3.4 \mathrm{mmol}, 1.5$ equiv), and $\mathrm{TMSCl}$ ( $0.35 \mathrm{~mL}, 2.8 \mathrm{mmol}, 1.3$ equiv). The title compound $4 \mathrm{q}$ was obtained as a pale yellow oil ( $0.440 \mathrm{~g}, 1.77 \mathrm{mmol}, 78 \%$ yield $)$. 
$R_{f}$ (silica, pentane): 0.7. ${ }^{1} \mathrm{H}$ NMR $\left(400 \mathrm{MHz}, \mathrm{CDCl}_{3}, \delta\right): 7.42$ $(\mathrm{m}, 2 \mathrm{H}), 7.31(\mathrm{~m}, 3 \mathrm{H}), 5.69(\mathrm{~s}, 1 \mathrm{H}), 5.46(\mathrm{~s}, 1 \mathrm{H}), 4.84$ (s, 4H), $0.04(\mathrm{~s}, 9 \mathrm{H})$ ppm. ${ }^{13} \mathrm{C}\left\{{ }^{1} \mathrm{H}\right\} \mathrm{NMR}\left(101 \mathrm{MHz}, \mathrm{CDCl}_{3}\right.$, $\delta): 149.52,137.8,128.3,128.0,127.2,114.2,84.0,78.1$, 1.5 ppm. HRMS (ESI): $\left[\mathrm{M}^{+}\right]$calcd. for $\mathrm{C}_{14} \mathrm{H}_{20} \mathrm{O}_{2} \mathrm{Si}, 248.1227$; found, 248.1224. IR (film): 3058 (w), 2958 (w), 2875 (w), 2251 (w), 1253 (m), 1153 (m), 991 (s), 908 (s), $843(\mathrm{~s}) \mathrm{cm}^{-1}$.

tert-Butyl 3-(1-Phenylvinyl)-3-((trimethylsilyl)oxy)azetidine1-carboxylate (4r).

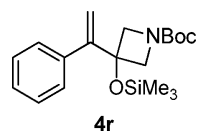

Compound $4 \mathbf{r}$ was prepared according to the general method $\mathrm{D}$ using $3 \mathrm{r}$ (0.44 g, $1.6 \mathrm{mmol}, 1.00$ equiv), $\mathrm{Et}_{3} \mathrm{~N}$ (0.33 mL, $2.4 \mathrm{mmol}$, 1.50 equiv), and TMSCl ( $0.24 \mathrm{~mL}, 1.9 \mathrm{mmol}, 1.2$ equiv). The title compound $4 \mathrm{r}$ was obtained as a colorless oil $(0.410 \mathrm{~g}, 1.18 \mathrm{mmol}$, $74 \%$ yield). ${ }^{1} \mathrm{H} \mathrm{NMR}\left(400 \mathrm{MHz}, \mathrm{CDCl}_{3}, \delta\right.$ ): 7.45 (dd, $J=8.2$, $1.5 \mathrm{~Hz}, 2 \mathrm{H}), 7.36-7.27(\mathrm{~m}, 3 \mathrm{H}), 5.61(\mathrm{~s}, 1 \mathrm{H}), 5.41(\mathrm{~s}, 1 \mathrm{H}), 4.18$ (s, 2H), 4.06 (dd, $J=9.0,0.9 \mathrm{~Hz}, 2 \mathrm{H}), 1.42(\mathrm{~s}, 9 \mathrm{H}), 0.04(\mathrm{~s}, 9 \mathrm{H})$ ppm. ${ }^{13} \mathrm{C}\left\{{ }^{1} \mathrm{H}\right\}$ NMR (101 MHz, $\left.\mathrm{CDCl}_{3}, \delta\right): 156.3,149.4,137.8$, 128.2, 127.8, 127.1, 114.2, 79.5, 73.8, 63.5, 62.0, 28.3, 1.3 ppm. HRMS (ESI): $[\mathrm{M}+\mathrm{H}]^{+}$calcd for $\mathrm{C}_{19} \mathrm{H}_{30} \mathrm{NO}_{3} \mathrm{Si}^{+}, 348.1989$; found, 348.1989.

(1-(3,4-Dihydronaphthalen-1-yl)cyclobutoxy)trimethylsilane (4s). Following a reported procedure, ${ }^{40} \mathrm{Br}_{2}(0.60 \mathrm{~mL}, 12 \mathrm{mmol}$, 1.25 equiv) was added dropwise to a solution of triphenyl phosphite $(2.7 \mathrm{~mL}, 10 \mathrm{mmol}, 1.1$ equiv) in anhydrous dichloromethane $(31.1 \mathrm{~mL})$ maintained at $-60{ }^{\circ} \mathrm{C}$ under Ar. A solution of $\mathrm{Et}_{3} \mathrm{~N}(1.7 \mathrm{~mL}, 13 \mathrm{mmol}$, 1.35 equiv) and 3,4-dihydronaphthalen- $1(2 \mathrm{H})$-one (26) $(1.2 \mathrm{~mL}$, $9.3 \mathrm{mmol}, 1.0$ equiv) in DCM $(5 \mathrm{~mL})$ was then added, and the resulting mixture was stirred for $18 \mathrm{~h}$, while allowing it to warm to room temperature, and then heated to reflux for another $2 \mathrm{~h}$. The reaction was quenched with saturated aqueous $\mathrm{Na}_{2} \mathrm{~S}_{2} \mathrm{O}_{3}(50 \mathrm{~mL})$. The organic layer was separated, and the aqueous layer was extracted with dichloromethane $(3 \times 30 \mathrm{~mL})$. The organic layers were combined, dried over anhydrous $\mathrm{Na}_{2} \mathrm{SO}_{4}$, filtered, and concentrated under reduced pressure. The residue was purified by column chromatography (silica, pentane) to give the 4-bromo-1,2-dihydronaphthalene (25s) (1.3 g, $6.2 \mathrm{mmol}, 67 \%)$ as a colorless oil. ${ }^{1} \mathrm{H}$ NMR $\left(400 \mathrm{MHz}, \mathrm{CDCl}_{3}, \delta\right): 7.57(\mathrm{dd}, J=7.6,1.4$, $1 \mathrm{H}), 7.24(\mathrm{~m}, 2 \mathrm{H}), 7.12(\mathrm{dd}, J=7.2,1.4,1 \mathrm{H}), 6.46(\mathrm{t}, J=4.8,1 \mathrm{H}), 2.86$ $(\mathrm{t}, J=8.1,2 \mathrm{H}), 2.43-2.34(\mathrm{~m}, 2 \mathrm{H}) \mathrm{ppm}$. The signals of the ${ }^{1} \mathrm{H}$ NMR spectra were in accordance with the data reported in the literature. ${ }^{40}$

Following a reported procedure, ${ }^{41} \mathrm{tBuLi}(2.0 \mathrm{M}$ in heptanes; $4.6 \mathrm{~mL}$, $9.2 \mathrm{mmol}, 2.0$ equiv) was added dropwise to a solution of 4-bromo-1,2dihydronaphthalene (25s) (0.96 g, $4.6 \mathrm{mmol})$ in anhydrous THF $(20 \mathrm{~mL})$ at $-78{ }^{\circ} \mathrm{C}$, over a period of $3 \mathrm{~min}$. The resultant colorless solution was stirred at $-78{ }^{\circ} \mathrm{C}$ for $0.5 \mathrm{~h}$. Cyclobutanone $(0.38 \mathrm{~mL}$, $5.1 \mathrm{mmol}, 1.1$ equiv) was then added, and the resulting mixture was stirred at $-78{ }^{\circ} \mathrm{C}$ for $0.5 \mathrm{~h}$. It was then allowed to slowly warm to room temperature. Water was added to quench the reaction, and the aqueous layer was extracted with EtOAc. The combined organic layers were dried over anhydrous $\mathrm{Na}_{2} \mathrm{SO}_{4}$, filtered, and concentrated under reduced pressure. The crude residue was then purified by flash chromatography (silica, pentane:EtOAc 100:0 to $95: 5$ to $85: 15)$ to give pure 1-(3,4dihydronaphthalen-1-yl)cyclobutanol (3s) (0.750 g, $3.74 \mathrm{mmol}, 81 \%)$ as a colorless solid. ${ }^{1} \mathrm{H}$ NMR $\left(400 \mathrm{MHz}, \mathrm{CDCl}_{3}, \delta\right): 7.51(\mathrm{~d}, J=7.3,1 \mathrm{H})$, $7.23-7.10(\mathrm{~m}, 3 \mathrm{H}), 6.20(\mathrm{t}, J=4.6,1 \mathrm{H}), 2.75(\mathrm{t}, J=8.0,2 \mathrm{H}), 2.61-2.47$ $(\mathrm{m}, 2 \mathrm{H}), 2.40-2.26(\mathrm{~m}, 4 \mathrm{H}), 2.08-1.88(\mathrm{~m}, 1 \mathrm{H}), 1.60(\mathrm{dtt}, J=11.0,8.8$, $7.5,1 \mathrm{H}), 0.93(\mathrm{~s}, 1 \mathrm{H}) \mathrm{ppm}$. The signals of the ${ }^{1} \mathrm{H}$ NMR spectra were in accordance with the data reported in the literature. ${ }^{41}$

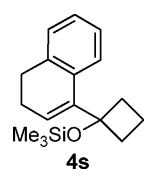

Compound $4 \mathbf{s}$ was prepared according to the general method $\mathrm{D}$ using $3 \mathrm{~s}\left(0.750 \mathrm{~g}, 3.74 \mathrm{mmol}, 1.0\right.$ equiv), $\mathrm{Et}_{3} \mathrm{~N}(0.78 \mathrm{~mL}$, $5.6 \mathrm{mmol}$, 1.5 equiv), and TMSCl $(0.57 \mathrm{~mL}, 4.5 \mathrm{mmol}$, 1.2 equiv). The title compound $4 \mathrm{~s}$ was obtained as a colorless oil (810 mg, $2.97 \mathrm{mmol}, 79 \%$ yield). ${ }^{1} \mathrm{H}$ NMR (400 MHz, $\mathrm{CDCl}_{3}$, $\delta): 7.57(\mathrm{dt}, J=6.1,1.8,1 \mathrm{H}), 7.20-7.06(\mathrm{~m}, 3 \mathrm{H}), 6.18(\mathrm{t}, J=$ 4.6, $1 \mathrm{H}), 2.79-2.68(\mathrm{~m}, 2 \mathrm{H}), 2.54-2.41(\mathrm{~m}, 2 \mathrm{H}), 2.40-2.25$ $(\mathrm{m}, 4 \mathrm{H}), 1.88-1.73(\mathrm{~m}, 1 \mathrm{H}), 1.56-1.40(\mathrm{~m}, 1 \mathrm{H}),-0.08(\mathrm{~s}, 9 \mathrm{H})$ ppm. ${ }^{13} \mathrm{C}\left\{{ }^{1} \mathrm{H}\right\}$ NMR $\left(101 \mathrm{MHz}, \mathrm{CDCl}_{3}, \delta\right): 139.2,137.1,132.8$, 127.3, 126.6, 126.3, 125.6, 124.4, 77.8, 37.0, 28.2, 23.3, 14.0, $1.5 \mathrm{ppm}$. HRMS (ESI): $\left[\mathrm{M}^{+}\right]$calcd for $\mathrm{C}_{17} \mathrm{H}_{24} \mathrm{OSi}, 272.1591$; found, 272.1589 .

Trimethyl(1-(1-(4-(trifluoromethyl)phenyl)vinyl)cyclobutoxy)silane $(4 t)$.

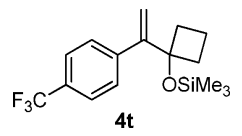

Compound $4 \mathbf{t}$ was prepared according to the general method $\mathrm{D}$ using $3 \mathrm{t}\left(1.00 \mathrm{~g}, 4.13 \mathrm{mmol}, 1.00\right.$ equiv), $\mathrm{Et}_{3} \mathrm{~N}(0.86 \mathrm{~mL}$, $6.2 \mathrm{mmol}, 1.5$ equiv), and TMSCl $(0.62 \mathrm{~mL}, 5.0 \mathrm{mmol}$, 1.3 equiv). The title compound $\mathbf{4 t}$ was obtained as a colorless oil (956 mg, $3.04 \mathrm{mmol}, 74 \%$ yield). $R_{f}$ (silica, pentane:EtOAc 99:1): 0.6. ${ }^{\mathrm{l}} \mathrm{H} \mathrm{NMR}\left(400 \mathrm{MHz}, \mathrm{CDCl}_{3}, \delta\right): 7.68-7.62(\mathrm{~m}, 2 \mathrm{H})$, $7.55(\mathrm{~d}, J=8.3 \mathrm{~Hz}, 2 \mathrm{H}), 5.52(\mathrm{~s}, 1 \mathrm{H}), 5.48(\mathrm{~s}, 1 \mathrm{H}), 2.41(\mathrm{dddd}$, $J=10.8,8.4,4.2,1.9 \mathrm{~Hz}, 2 \mathrm{H}), 2.37-2.28(\mathrm{~m}, 2 \mathrm{H}), 1.92-1.72$ $(\mathrm{m}, 1 \mathrm{H}), 1.54(\mathrm{dt}, J=10.9,8.7 \mathrm{~Hz}, 1 \mathrm{H}), 0.00(\mathrm{~s}, 9 \mathrm{H}) \mathrm{ppm}$. ${ }^{13} \mathrm{C}\left\{{ }^{1} \mathrm{H}\right\}$ NMR $\left(101 \mathrm{MHz}, \mathrm{CDCl}_{3}, \delta\right): 150.78,143.1,129.1$ $(\mathrm{q}, J=32.4 \mathrm{~Hz}), 128.2,124.6(\mathrm{~d}, J=3.9 \mathrm{~Hz}), 124.3(\mathrm{q}, J=$ $101.5 \mathrm{~Hz}), 113.7,78.56,36.9,13.5,1.6 \mathrm{ppm} .{ }^{19} \mathrm{~F}\left\{{ }^{1} \mathrm{H}\right\}$ NMR $\left(376 \mathrm{MHz}, \mathrm{CDCl}_{3}, \delta\right):-62.49$ ppm. HRMS (ESI): $[\mathrm{M}]^{+}$calcd for $\mathrm{C}_{16} \mathrm{H}_{21} \mathrm{~F}_{3} \mathrm{OSi}$, 314.1308; found, 314.1314. IR (film): 2987 (s), $2901(\mathrm{~m}), 1619(\mathrm{w}), 1407(\mathrm{~m}), 1326(\mathrm{~s}), 1252(\mathrm{~s}), 1165$ (m), $1123(\mathrm{~s}), 1068(\mathrm{~s}), 991(\mathrm{~m}), 840(\mathrm{~s}), 754(\mathrm{w}) \mathrm{cm}^{-1}$.

Ring Expansion Reactions. General Procedure E. Freshly dried $\mathrm{CH}_{3} \mathrm{CN}(2.5 \mathrm{~mL}, 0.20 \mathrm{M})$ was added to a mixture of (1-(1(arene)vinyl)cyclobutoxy) trimethylsilane $4(0.5 \mathrm{mmol}, 1$ equiv), $2\left(0.27 \mathrm{mg}, 0.60 \mathrm{mmol}, 1.2\right.$ equiv), and $\mathrm{Cu}(\mathrm{dap})_{2} \mathrm{Cl}(2.2 \mathrm{mg}, 2.5 \mu \mathrm{mol}$, $5 \mathrm{~mol} \%$ ), and the mixture was degassed (three pump and freeze cycles) and stirred for $16 \mathrm{~h}$ at room temperature, while being irradiated with green LEDs. Thereafter, the mixture was filtered through a plug of Celite and concentrated. The residue was purified by column chromatography (silica, pentane:EtOAc 30:1), affording the title compounds 6 .

2-(Azidomethyl)-2-phenylcyclopentanone (6a).<smiles>NCC1(c2ccccc2)CCCC1=O</smiles>

Compound 6a was prepared according to general method $\mathrm{E}$ using $4 \mathrm{a}(246 \mathrm{mg}, 1.00 \mathrm{mmol}, 1.0$ equiv), $2(531 \mathrm{mg}, 1.20 \mathrm{mmol}$, 1.2 equiv), and $\mathrm{Cu}(\text { dap })_{2} \mathrm{Cl}(4.4 \mathrm{mg}, 5.0 \mu \mathrm{mol}, 0.5 \mathrm{~mol} \%)$ in $\mathrm{CH}_{3} \mathrm{CN}(5.0 \mathrm{~mL}, 0.2 \mathrm{~m})$. The title compound $\mathbf{6 a}$ was obtained as a colorless oil $(193 \mathrm{mg}, 0.897 \mathrm{mmol}, 90 \%) . R_{f} 0.7$ (silica, pentane:EtOAc 10:1). ${ }^{1} \mathrm{H}$ NMR $\left(400 \mathrm{MHz}, \mathrm{CDCl}_{3}, \delta\right): 7.37$ $(\mathrm{m}, 4 \mathrm{H}), 7.30(\mathrm{~m}, 1 \mathrm{H}), 3.72(\mathrm{~d}, J=12.2 \mathrm{~Hz}, 1 \mathrm{H}), 3.49(\mathrm{~d}, J=$ $12.2 \mathrm{~Hz}, 1 \mathrm{H}), 2.60(\mathrm{~m}, 1 \mathrm{H}), 2.41-2.19(\mathrm{~m}, 3 \mathrm{H}), 2.00(\mathrm{~m}, 1 \mathrm{H})$, $1.78(\mathrm{~m}, 1 \mathrm{H}) \mathrm{ppm} .{ }^{13} \mathrm{C}\left\{{ }^{1} \mathrm{H}\right\} \mathrm{NMR}\left(101 \mathrm{MHz}, \mathrm{CDCl}_{3}, \delta\right): 217.5$, $136.9,129.0,127.8,126.8,57.9,57.8,37.5,31.7,18.5 \mathrm{ppm}$. HRMS (ESI): $[\mathrm{M}+\mathrm{Na}]^{+}$calcd. for $\mathrm{C}_{12} \mathrm{H}_{13} \mathrm{~N}_{3} \mathrm{NaO}^{+}, 238.0951$; found, 238.0953. IR (film): 2967 (w), 2925 (w), 2917 (w), 2890 $(\mathrm{w}), 2104(\mathrm{~s}), 1738(\mathrm{~s}), 1497(\mathrm{w}), 1448(\mathrm{w}), 1301(\mathrm{w})$, 
$1300(w), 1288(w), 1253(w), 1160(w) \mathrm{cm}^{-1}$. NMR data correspond to the reported values. ${ }^{37}$

Characterization of 2-(Azidomethyl)-2-phenyl-1-oxaspiro[2.3]hexane (7a).

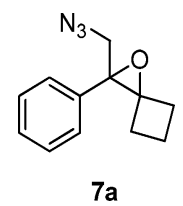

$R_{f} 0.45$ (silica, pentane:EtOAc 10:1). ${ }^{1} \mathrm{H}$ NMR (400 MHz, $\left.\mathrm{CDCl}_{3}, \delta\right): 7.41-7.29(\mathrm{~m}, 5 \mathrm{H}), 3.87(\mathrm{~d}, J=13.3 \mathrm{~Hz}, 1 \mathrm{H}), 3.48$ $(\mathrm{d}, J=13.3 \mathrm{~Hz}, 1 \mathrm{H}), 2.67-2.55(\mathrm{~m}, 1 \mathrm{H}), 2.53-2.44(\mathrm{~m}, 1 \mathrm{H})$, $2.32(\mathrm{~m}, 1 \mathrm{H}), 1.98-1.82(\mathrm{~m}, 2 \mathrm{H}), 1.78-1.68(\mathrm{~m}, 1 \mathrm{H}) \mathrm{ppm}$. ${ }^{13} \mathrm{C}\left\{{ }^{1} \mathrm{H}\right\}$ NMR $\left(101 \mathrm{MHz}, \mathrm{CDCl}_{3}, \delta\right): 136.2,128.5,128.1$, 126.6, 69.7, 65.5, 54.7, 29.6, 29.3, 12.9 ppm. HRMS (ESI): $\left[\mathrm{M}+\mathrm{H}-\mathrm{N}_{2}\right]^{+}$calcd. for $\mathrm{C}_{12} \mathrm{H}_{14} \mathrm{NO}^{+}, 188.1070$; found, 188.1071. IR (film): 2987 (s), 2972 (s), 2902 (m), 2105 (m), $1408(\mathrm{~m}), 1329(\mathrm{~s}), 1251(\mathrm{w}), 1168(\mathrm{~m}), 1127(\mathrm{~m})$, $1067(\mathrm{~s}), 894(\mathrm{w}), 846(\mathrm{w}) \mathrm{cm}^{-1}$.

2-(Azidomethyl)-2-(p-tolyl)cyclopentanone (6b).

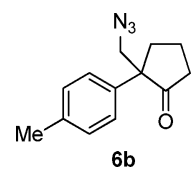

Compound $\mathbf{6 b}$ was prepared according to general method $\mathrm{E}$ using 4b (130 mg, $0.500 \mathrm{mmol}, 1.00$ equiv), 2 (265 mg, $0.600 \mathrm{mmol}, 1.20$ equiv), and $\mathrm{Cu}(\mathrm{dap})_{2} \mathrm{Cl}(2.2 \mathrm{mg}, 2.5 \mu \mathrm{mol}$, $0.50 \mathrm{~mol} \%)$ in $\mathrm{CH}_{3} \mathrm{CN}(2.5 \mathrm{~mL}, 0.20 \mathrm{~m})$. The title compound 6b was obtained as a colorless oil (103 $\mathrm{mg}, 0.449 \mathrm{mmol}, 90 \%)$. $R_{f} 0.7$ (silica, pentane:EtOAc 10:1). ${ }^{1} \mathrm{H}$ NMR (400 MHz, $\left.\mathrm{CDCl}_{3}, \delta\right): 7.28(\mathrm{~d}, J=8.2 \mathrm{~Hz}, 2 \mathrm{H}, \mathrm{ArH}), 7.18(\mathrm{~d}, J=8.2 \mathrm{~Hz}$, $2 \mathrm{H}, \operatorname{ArH}), 3.72\left(\mathrm{~d}, J=12.2 \mathrm{~Hz}, 1 \mathrm{H}, \mathrm{CH}_{2}-\mathrm{N}_{3}\right), 3.47(\mathrm{~d}, J=$ $\left.12.2 \mathrm{~Hz}, 1 \mathrm{H}, \mathrm{CH}_{2}-\mathrm{N}_{3}\right), 2.60\left(\mathrm{~m}, 1 \mathrm{H}, \mathrm{CH}_{2}\right), 2.42-2.16(\mathrm{~m}, 6 \mathrm{H}$, $\mathrm{CH}_{3}$ and $\left.\mathrm{CH}_{2}\right), 2.04-1.92\left(\mathrm{~m}, 1 \mathrm{H}, \mathrm{CH}_{2}\right), 1.79\left(\mathrm{~m}, 1 \mathrm{H}, \mathrm{CH}_{2}\right)$ ppm. ${ }^{13} \mathrm{C}\left\{{ }^{1} \mathrm{H}\right\}$ NMR $\left(101 \mathrm{MHz}, \mathrm{CDCl}_{3}, \delta\right): 217.5,137.5$, 133.6, 129.6, 126.6, 57.8, 57.4, 37.3, 31.6, 20.9, 18.4 ppm. HRMS (ESI): $[\mathrm{M}+\mathrm{Na}]^{+}$calcd. for $\mathrm{C}_{13} \mathrm{H}_{15} \mathrm{~N}_{3} \mathrm{NaO}^{+}, 252.1107$; found, 252.1114. IR (film): 2968 (w), 2924 (w), 2886 (w), 2101 (s), 1737 (s), 1515 (w), $1456(\mathrm{w}), 1299$ (w), 1279 (w), $1163(\mathrm{w}), 1022(\mathrm{w}), 908(\mathrm{w}), 817(\mathrm{w}) \mathrm{cm}^{-1}$.

2-(Azidomethyl)-2-(4-(tert-butyl)phenyl)cyclopentanone $(6 c)$.

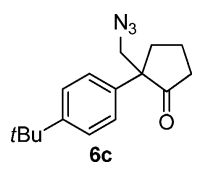

Compound $6 \mathrm{c}$ was prepared according to general method $\mathrm{E}$ using 4c (151 mg, $0.500 \mathrm{mmol}, 1.00$ equiv), 2 (265 mg, 0.600 mmol, 1.20 equiv), and $\mathrm{Cu}(\text { dap })_{2} \mathrm{Cl}(2.2 \mathrm{mg}, 2.5 \mu \mathrm{mol}, 0.5$ $\mathrm{mol} \%)$ in $\mathrm{CH}_{3} \mathrm{CN}(2.5 \mathrm{~mL}, 0.2 \mathrm{~m})$. The title compound $6 \mathrm{c}$ was obtained as a colorless oil (115 mg, $0.424 \mathrm{mmol}, 85 \%) . R_{f}$ 0.7 (silica, pentane:EtOAc 10:1). ${ }^{1} \mathrm{H} \mathrm{NMR}\left(400 \mathrm{MHz} \mathrm{CDCl}_{3}\right.$, $\delta): 7.39-7.34(\mathrm{~m}, 2 \mathrm{H}), 7.32-7.27(\mathrm{~m}, 2 \mathrm{H}), 3.74(\mathrm{~d}, J=12.3$ $\mathrm{Hz}, 1 \mathrm{H}), 3.46$ (d, $J=12.3 \mathrm{~Hz}, 1 \mathrm{H}), 2.64-2.56(\mathrm{~m}, 1 \mathrm{H}), 2.40-$ $2.18(\mathrm{~m}, 3 \mathrm{H}), 2.07-1.92(\mathrm{~m}, 1 \mathrm{H}), 1.87-1.72(\mathrm{~m}, 1 \mathrm{H}), 1.30$ (s, 9H) ppm. ${ }^{13} \mathrm{C}\left\{{ }^{1} \mathrm{H}\right\}$ NMR (101 MHz, $\left.\mathrm{CDCl}_{3}, \delta\right): 217.5$, 150.6, 133.6, 126.3, 125.8, 57.7, 57.3, 37.4, 34.3, 31.5, 31.2, 18.4 ppm. HRMS (ESI): $[\mathrm{M}+\mathrm{Na}]^{+}$calcd. for $\mathrm{C}_{16} \mathrm{H}_{21} \mathrm{~N}_{3} \mathrm{NaO}^{+}$, 294.1577; found, 294.1584. IR (film): $2964(\mathrm{~m}), 2907$ (w), $2870(\mathrm{w}), 2597$ (w), 2104 (s), 1739 (m), $1510(w), 1464(w), 1405(w), 1365(w), 1272(w), 1160(w)$ $1018(\mathrm{w}), 836(\mathrm{w}) \mathrm{cm}^{-1}$.

2-([1,1'-Biphenyl]-4-yl)-2-(azidomethyl)cyclopentanone (6d).

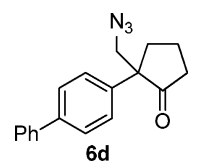

Compound 6d was prepared according to general method $\mathrm{E}$ using 4d (161 mg, $0.500 \mathrm{mmol}, 1.00$ equiv), 2 (265 mg, $0.600 \mathrm{mmol}, 1.20$ equiv), and $\mathrm{Cu}(\mathrm{dap})_{2} \mathrm{Cl}(2.2 \mathrm{mg}, 2.5 \mu \mathrm{mol}$, $0.5 \mathrm{~mol} \%)$ in $\mathrm{CH}_{3} \mathrm{CN}(2.5 \mathrm{~mL}, 0.2 \mathrm{~m})$. The title compound 6d was obtained as a colorless oil (130 mg, $0.446 \mathrm{mmol}, 89 \%)$. $R_{f} 0.7$ (silica, pentane:EtOAc 10:1). ${ }^{1} \mathrm{H}$ NMR (400 MHz, $\left.\mathrm{CDCl}_{3}, \delta\right): 7.63-7.55(\mathrm{~m}, 4 \mathrm{H}), 7.51-7.42(\mathrm{~m}, 4 \mathrm{H}), 7.40-$ $7.33(\mathrm{~m}, 1 \mathrm{H}), 3.78(\mathrm{~d}, J=12.2 \mathrm{~Hz}, 1 \mathrm{H}), 3.53(\mathrm{~d}, J=12.3 \mathrm{~Hz}$, $1 \mathrm{H}), 2.73-2.59(\mathrm{~m}, 1 \mathrm{H}), 2.45-2.22(\mathrm{~m}, 3 \mathrm{H}), 2.10-1.98$ $\left(\mathrm{m}, 1 \mathrm{H}_{2}\right), 1.93-1.76(\mathrm{~m}, 1 \mathrm{H}) \mathrm{ppm} .{ }^{13} \mathrm{C}\left\{{ }^{1} \mathrm{H}\right\}$ NMR $(101 \mathrm{MHz}$, $\left.\mathrm{CDCl}_{3}, \delta\right): 217.3,140.5,140.1,135.7,128.7,127.5,127.4$, 127.1, 126.9, 57.7, 57.4, 37.4, 31.6, 18.4 ppm. HRMS (ESI): $[\mathrm{M}+\mathrm{Na}]^{+}$calcd. for $\mathrm{C}_{18} \mathrm{H}_{17} \mathrm{~N}_{3} \mathrm{NaO}^{+}, 314.1264$; found, 314.1266. IR (film): 3033 (w), 2973 (w), 2881 (w), 2103 (s), 1737 (s), $1601(\mathrm{w}), 1523(\mathrm{w}), 1489(\mathrm{w}), 1406(\mathrm{w}), 1301(\mathrm{w})$, 1271 (w), 1159 (w), 1009 (w), $842(w) \mathrm{cm}^{-1}$.

2-(Azidomethyl)-2-(4-methoxyphenyl)cyclopentanone (6e).<smiles>COc1ccc(C2(CN)CCCC2=O)cc1</smiles>

Compound 6e was prepared according to general method $\mathrm{E}$ using $4 \mathrm{e}$ (138 mg, $0.500 \mathrm{mmol}, 1.00$ equiv), $2(265 \mathrm{mg}$, $0.600 \mathrm{mmol}, 1.20$ equiv), and $\mathrm{Cu}(\mathrm{dap})_{2} \mathrm{Cl}(2.2 \mathrm{mg}, 2.5 \mu \mathrm{mol}$, $0.5 \mathrm{~mol} \%)$ in $\mathrm{CH}_{3} \mathrm{CN}(2.5 \mathrm{~mL}, 0.2 \mathrm{~m})$. The title compound 6e was obtained as a colorless oil (112 mg, $0.457 \mathrm{mmol}, 91 \%)$. $R_{f} 0.5$ (silica, pentane:EtOAc 10:1). ${ }^{1} \mathrm{H}$ NMR (400 MHz, $\left.\mathrm{CDCl}_{3}, \delta\right): 7.35-7.19(\mathrm{~m}, 2 \mathrm{H}), 6.95-6.79(\mathrm{~m}, 2 \mathrm{H}), 3.77$ (s, 3H), $3.67(\mathrm{~d}, J=12.3 \mathrm{~Hz}, 1 \mathrm{H}), 3.43(\mathrm{~d}, J=12.2 \mathrm{~Hz}, 1 \mathrm{H})$, $2.56(\mathrm{~m}, 1 \mathrm{H}), 2.40-2.13(\mathrm{~m}, 3 \mathrm{H}), 2.03-1.88(\mathrm{~m}, 1 \mathrm{H}), 1.86-$ $1.67(\mathrm{~m}, 1 \mathrm{H}) \mathrm{ppm} .{ }^{13} \mathrm{C}\left\{{ }^{1} \mathrm{H}\right\}$ NMR $\left(101 \mathrm{MHz}, \mathrm{CDCl}_{3}, \delta\right)$ : 217.4, 159.0, 128.3, 127.8, 114.2, 57.7, 56.9, 55.1, 37.2, 31.5, 18.3 ppm. HRMS (ESI): $[\mathrm{M}+\mathrm{Na}]^{+}$calcd. for $\mathrm{C}_{13} \mathrm{H}_{15} \mathrm{~N}_{3} \mathrm{NaO}_{2}{ }^{+}$, 268.1056; found, 268.1055. IR (film): 2961 (w), 2838 (w), 2101 (s), 1734 (s), 1609 (w), 1512 (s), 1464 (w), 1253 (s), $1187(\mathrm{~m}), 1034(\mathrm{~m}), 905(\mathrm{~m}), 832(\mathrm{~m}) \mathrm{cm}^{-1}$.

2-(Azidomethyl)-2-(4-fluorophenyl)cyclopentanone (6f).

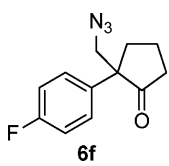

Compound $6 \mathrm{f}$ was prepared according to general method $\mathrm{E}$ using 4f (132 mg, $0.500 \mathrm{mmol}, 1.00$ equiv), 2 (265 mg, $0.600 \mathrm{mmol}, 1.20$ equiv), and $\mathrm{Cu}(\text { dap })_{2} \mathrm{Cl}(2.2 \mathrm{mg}, 2.5 \mu \mathrm{mol}$, $0.5 \mathrm{~mol} \%)$ in $\mathrm{CH}_{3} \mathrm{CN}(2.5 \mathrm{~mL}, 0.2 \mathrm{~m})$. The title compound $6 \mathrm{f}$ was obtained as a colorless oil ( $98 \mathrm{mg}, 0.42 \mathrm{mmol}, 84 \%) . R_{f} 0.7$ (silica, pentane:EtOAc 10:1). ${ }^{1} \mathrm{H}$ NMR $\left(400 \mathrm{MHz}, \mathrm{CDCl}_{3}, \delta\right)$ : 7.49-7.28 (m, 2H), 7.11-6.93 (m, 2H), $3.66(\mathrm{~d}, J=12.3 \mathrm{~Hz}$, $1 \mathrm{H}), 3.45(\mathrm{~d}, J=12.3 \mathrm{~Hz}, 1 \mathrm{H}), 2.56(\mathrm{~m}, 1 \mathrm{H}), 2.46-2.16(\mathrm{~m}$, $3 \mathrm{H}), 2.13-1.89(\mathrm{~m}, 1 \mathrm{H}), 1.76(\mathrm{~m}, 1 \mathrm{H}) \mathrm{ppm} .{ }^{13} \mathrm{C}\left\{{ }^{1} \mathrm{H}\right\}$ NMR $\left(101 \mathrm{MHz}, \mathrm{CDCl}_{3}, \delta\right): 217.2,162.16(\mathrm{~d}, J=247.4 \mathrm{~Hz}), 132.48$ $(\mathrm{d}, J=3.3 \mathrm{~Hz}), 128.50(\mathrm{~d}, J=8.1 \mathrm{~Hz}), 115.75(\mathrm{~d}, J=21.3 \mathrm{~Hz})$, 
57.8, 57.0, 37.4, 31.8, 18.4 ppm. ${ }^{19} \mathrm{~F}\left\{{ }^{1} \mathrm{H}\right\}$ NMR (376 MHz, $\left.\mathrm{CDCl}_{3}\right): \delta=-114.44(\mathrm{tt}, J=8.2,5.2 \mathrm{~Hz}$ ) ppm. HRMS (ESI): $[\mathrm{M}+\mathrm{Na}]^{+}$calcd. for $\mathrm{C}_{12} \mathrm{H}_{12} \mathrm{FN}_{3} \mathrm{NaO}^{+}, 256.0857$; found, 256.0857. IR (film): 2977 (w), 2255 (w), 2105 (s), 1737 (m), $1605(\mathrm{w}), 1510(\mathrm{~m}), 1405(\mathrm{w}), 1284(\mathrm{w}), 1239(\mathrm{~m}), 1167$ (w), $1110(\mathrm{w}), 1016(\mathrm{w}), 908$ (s), 837 (w) $\mathrm{cm}^{-1}$.

2-(Azidomethyl)-2-(4-chlorophenyl)cyclopentanone (6g).

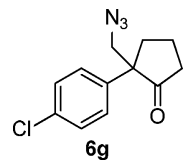

Compound $6 \mathrm{~g}$ was prepared according to general method $\mathrm{E}$ using $4 \mathrm{~g}$ (140 mg, $0.500 \mathrm{mmol}, 1.00$ equiv), 2 (265 mg, $0.600 \mathrm{mmol}, 1.20$ equiv), and $\mathrm{Cu}(\text { dap })_{2} \mathrm{Cl}(2.2 \mathrm{mg}, 2.5 \mu \mathrm{mol}$, $0.5 \mathrm{~mol} \%)$ in $\mathrm{CH}_{3} \mathrm{CN}(2.5 \mathrm{~mL}, 0.2 \mathrm{~m})$. The title compound $\mathbf{6 g}$ was obtained as a colorless oil $(120 \mathrm{mg}, 0.481 \mathrm{mmol}, 96 \%)$. $R_{f} 0.7$ (silica, pentane:EtOAc 10:1). ${ }^{1} \mathrm{H}$ NMR (400 MHz, $\left.\mathrm{CDCl}_{3}, \delta\right): 7.35-7.28(\mathrm{~m}, 4 \mathrm{H}), 3.66(\mathrm{~d}, J=12.3 \mathrm{~Hz}, 1 \mathrm{H}), 3.44$ $(\mathrm{d}, J=12.3 \mathrm{~Hz}, 1 \mathrm{H}), 2.59-2.49(\mathrm{~m}, 1 \mathrm{H}), 2.39-2.18(\mathrm{~m}, 3 \mathrm{H})$, 2.04-1.93 (m, 1H), 1.82-1.67 (m, 1H) ppm. ${ }^{13} \mathrm{C}\left\{{ }^{1} \mathrm{H}\right\} \mathrm{NMR}$ $\left(101 \mathrm{MHz}, \mathrm{CDCl}_{3}, \delta\right): 216.9,135.3,133.7,128.9,128.1,57.6$, 57.0, 37.4, 31.6, 18.4 ppm. HRMS (ESI): $[\mathrm{M}+\mathrm{Na}]^{+}$calcd. for $\mathrm{C}_{12} \mathrm{H}_{12} \mathrm{ClN}_{3} \mathrm{NaO}^{+}$, 272.0561; found, 272.0563. IR (film): 2971 (w), $2885(\mathrm{w}), 2105$ (s), $1739(\mathrm{~m}), 1494(\mathrm{~m}), 1404(\mathrm{w}), 1276$ (w), 1159 (w), $1097(\mathrm{w}), 1014(\mathrm{~m}), 832(\mathrm{w}) \mathrm{cm}^{-1}$.

2-(Azidomethyl)-2-(m-tolyl)cyclopentanone (6h).<smiles>Cc1cccc(C2(CN)CCCC2=O)c1</smiles>

Compound $\mathbf{6 h}$ was prepared according to general method E using 4h (130 mg, $0.500 \mathrm{mmol}, 1.00$ equiv), 2 (265 mg, $0.600 \mathrm{mmol}, 1.20$ equiv), and $\mathrm{Cu}(\mathrm{dap})_{2} \mathrm{Cl}(2.2 \mathrm{mg}, 2.5 \mu \mathrm{mol}$, $0.5 \mathrm{~mol} \%)$ in $\mathrm{CH}_{3} \mathrm{CN}(2.5 \mathrm{~mL}, 0.2 \mathrm{~m})$. The title compound 6h was obtained as a colorless oil (105 mg, $0.458 \mathrm{mmol}, 92 \%)$. $R_{f} 0.7$ (silica, pentane:EtOAc 10:1). ${ }^{1} \mathrm{H}$ NMR (400 MHz, $\left.\mathrm{CDCl}_{3}, \delta\right): 7.27-7.21(\mathrm{~m}, 1 \mathrm{H}, \mathrm{ArH}), 7.20-7.15(\mathrm{~m}, 2 \mathrm{H}$, $\operatorname{ArH}$ ), 7.10 (d, $J=7.5 \mathrm{~Hz}, 1 \mathrm{H}, \mathrm{ArH}), 3.72(\mathrm{~d}, J=12.3 \mathrm{~Hz}, 1 \mathrm{H}$, $\mathrm{CH}_{2}-\mathrm{N}_{3}$ ), 3.47 (d, $\mathrm{J}=12.3 \mathrm{~Hz}, 1 \mathrm{H}, \mathrm{CH}_{2}-\mathrm{N}_{3}$ ), $2.60(\mathrm{~m}, 1 \mathrm{H}$, $\left.\mathrm{CH}_{2}\right), 2.44-2.17\left(\mathrm{~m}, 6 \mathrm{H}, \mathrm{CH}_{3}, \mathrm{CH}_{2}\right), 1.99\left(\mathrm{~m}, 1 \mathrm{H}, \mathrm{CH}_{2}\right)$, 1.86-1.69 (m, 1H, CH $)$ ppm. ${ }^{13} \mathrm{C}\left\{{ }^{1} \mathrm{H}\right\}$ NMR (101 MHz, $\left.\mathrm{CDCl}_{3}, \delta\right): 217.4,138.5,136.7,128.7,128.4,127.3,123.5$, 57.7, 57.6, 37.3, 31.6, 21.4, 18.3 ppm. HRMS (ESI): $[\mathrm{M}+$ $\mathrm{Na}]^{+}$calcd. for $\mathrm{C}_{13} \mathrm{H}_{15} \mathrm{~N}_{3} \mathrm{NaO}^{+}$, 252.1107; found, 252.1111. IR (film): $3458(\mathrm{w}), 3027(\mathrm{w}), 2968(\mathrm{w}), 2886(\mathrm{w}), 2253(\mathrm{w})$, 2101 (s), 1736 (s), 1605 (w), $1456(\mathrm{w}), 1300$ (w), $1272(\mathrm{w})$, $1158(\mathrm{w}), 909(\mathrm{~m}) \mathrm{cm}^{-1}$.

\section{2-(Azidomethyl)-2-(3-methoxyphenyl)cyclopentanone (6i).}

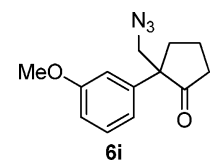

Compound 6i was prepared according to general method E using 4i (138 mg, $0.500 \mathrm{mmol}, 1.00$ equiv), 2 (265 mg, $0.600 \mathrm{mmol}, 1.20$ equiv), and $\mathrm{Cu}(\text { dap })_{2} \mathrm{Cl}(2.2 \mathrm{mg}, 2.5 \mu \mathrm{mol}$, $0.5 \mathrm{~mol} \%)$ in $\mathrm{CH}_{3} \mathrm{CN}(2.5 \mathrm{~mL}, 0.2 \mathrm{~m})$. The title compound $6 \mathbf{i}$ was obtained as a colorless oil (105 $\mathrm{mg}, 0.428 \mathrm{mmol}, 86 \%)$. $R_{f} 0.6$ (silica, pentane:EtOAc 10:1). ${ }^{1} \mathrm{H}$ NMR $(400 \mathrm{MHz}$, $\left.\mathrm{CDCl}_{3}, \delta\right): 7.27(\mathrm{t}, J=8.0 \mathrm{~Hz}, 1 \mathrm{H}), 6.95(\mathrm{ddd}, J=8.0,2.2$,
$0.9 \mathrm{~Hz}, 1 \mathrm{H}), 6.92(\mathrm{t}, J=2.2 \mathrm{~Hz}, 1 \mathrm{H}), 6.83(\mathrm{ddd}, J=8.0,2.2$, $0.9 \mathrm{~Hz}, 1 \mathrm{H}), 3.80(\mathrm{~s}, 3 \mathrm{H}), 3.71(\mathrm{~d}, \mathrm{~J}=12.2 \mathrm{~Hz}, 1 \mathrm{H}), 3.48$ $(\mathrm{d}, J=12.2 \mathrm{~Hz}, 1 \mathrm{H}), 2.64-2.52(\mathrm{~m}, 1 \mathrm{H}), 2.41-2.16(\mathrm{~m}, 3 \mathrm{H})$, 2.07-1.93 (m, 1H), 1.87-1.71 (m, 1H) ppm. ${ }^{13} \mathrm{C}\left\{{ }^{1} \mathrm{H}\right\}$ NMR $\left(101 \mathrm{MHz}, \mathrm{CDCl}_{3}, \delta\right): 217.3,159.9,138.4,129.9,118.9,113.0$, $112.7,57.7,55.2,37.4,31.7,18.4$ ppm. HRMS (ESI): $\left[\mathrm{M}^{+}\right]$ calcd for $\mathrm{C}_{13} \mathrm{H}_{15} \mathrm{NO}_{2}, 217.1097$; found, 217.1101 (loss of $\mathrm{N}_{2}$ ). IR (film): 2968 (w), 2837 (w), 2102 (s), 1736 (s), 1599 (m), $1582(\mathrm{~m}), 1490(\mathrm{~m}), 1435(\mathrm{w}), 1292(\mathrm{~m}), 1263(\mathrm{~m}), 1242$ (m), $1157(\mathrm{w}), 1053(\mathrm{~m}), 883(\mathrm{w}), 781(\mathrm{w}) \mathrm{cm}^{-1}$.

2-(Azidomethyl)-2-(3-fluorophenyl)cyclopentanone (6j).

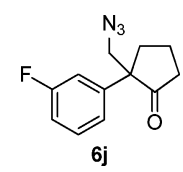

Compound $6 \mathbf{j}$ was prepared according to general method $\mathrm{E}$ using $4 \mathrm{j}$ (132 mg, $0.500 \mathrm{mmol}, 1.00$ equiv), 2 (265 mg, $0.600 \mathrm{mmol}, 1.20$ equiv), and $\mathrm{Cu}(\text { dap })_{2} \mathrm{Cl}(2.2 \mathrm{mg}, 2.5 \mu \mathrm{mol}$, $0.5 \mathrm{~mol} \%)$ in $\mathrm{CH}_{3} \mathrm{CN}(2.5 \mathrm{~mL}, 0.2 \mathrm{~m})$. The title compound $\mathbf{6 j}$ was obtained as a colorless oil $(96 \mathrm{mg}, 0.41 \mathrm{mmol}, 82 \%) . R_{f} 0.6$ (silica, pentane:EtOAc 10:1). ${ }^{1} \mathrm{H}$ NMR $\left(400 \mathrm{MHz} \mathrm{CDCl}_{3}, \delta\right)$ : $7.33(\mathrm{td}, J=8.0,6.1 \mathrm{~Hz}, 1 \mathrm{H}), 7.17(\mathrm{dt}, J=8.0,1.2 \mathrm{~Hz}, 1 \mathrm{H})$, $7.12(\mathrm{dt}, J=10.5,2.2 \mathrm{~Hz}, 1 \mathrm{H}), 7.03-6.95(\mathrm{~m}, 1 \mathrm{H}), 3.69(\mathrm{~d}$, $J=12.3 \mathrm{~Hz}, 1 \mathrm{H}), 3.48(\mathrm{~d}, J=12.3 \mathrm{~Hz}, 1 \mathrm{H}), 2.62-2.52$ (m, 1H), 2.43-2.23 (m, 3H), 2.09-1.96 (m, 1H), 1.87-1.70 $(\mathrm{m}, 1 \mathrm{H})$ ppm. ${ }^{13} \mathrm{C}\left\{{ }^{1} \mathrm{H}\right\}$ NMR $\left(101 \mathrm{MHz}, \mathrm{CDCl}_{3}, \delta\right):$ 216.9, $163.0(\mathrm{~d}, J=246.8 \mathrm{~Hz}), 139.5(\mathrm{~d}, J=7.0 \mathrm{~Hz}), 130.4(\mathrm{~d}, J=$ $8.3 \mathrm{~Hz}), 122.4(\mathrm{~d}, J=2.9 \mathrm{~Hz}), 114.8(\mathrm{~d}, J=21.1 \mathrm{~Hz}), 114.0(\mathrm{~d}$, $J=22.6 \mathrm{~Hz}$ ), 57.7, 57.5 (d, $J=1.7 \mathrm{~Hz}), 37.5,31.8,18.5 \mathrm{ppm}$. ${ }^{19} \mathrm{~F}$ NMR (376 MHz, $\mathrm{CDCl}_{3}, \delta$ ): -111.5 (ddd, $J=10.7,7.7$, $6.1 \mathrm{~Hz})$ ppm. HRMS (ESI): $[\mathrm{M}+\mathrm{H}]^{+}$calcd. for $\mathrm{C}_{12} \mathrm{H}_{13} \mathrm{FNO}^{+}$, 206.0976; found, 206.0973 (loss of $\mathrm{N}_{2}$ ). IR (film): 2972 (w), 2105 (s), 1738 (m), 1614 (w), 1588 (w), 1490 (w), 1439 (w), $1274(\mathrm{w}), 1161(\mathrm{w}), 875(\mathrm{w}), 789(\mathrm{w}) \cdot \mathrm{cm}^{-1}$.

2-(Azidomethyl)-2-(o-tolyl)cyclopentanone (6k).

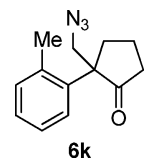

Compound 6k was prepared according to general method $\mathrm{E}$ using 4k ( $0.13 \mathrm{~g}, 0.50 \mathrm{mmol}, 1$ equiv), 2 ( $265 \mathrm{mg}, 0.600 \mathrm{mmol}$, 1.20 equiv), and $\mathrm{Cu}(\mathrm{dap})_{2} \mathrm{Cl}(2.2 \mathrm{mg}, 2.5 \mu \mathrm{mol}, 0.5 \mathrm{~mol} \%)$ in $\mathrm{CH}_{3} \mathrm{CN}(2.5 \mathrm{~mL}, 0.2 \mathrm{~m})$. The title compound $6 \mathrm{k}$ was obtained as a colorless oil $(89 \mathrm{mg}, 0.39 \mathrm{mmol}, 78 \%) . R_{f} 0.7$ (silica, pentane:EtOAc 10:1). ${ }^{1} \mathrm{H}$ NMR (400 MHz, $\mathrm{CDCl}_{3}, \delta$ ): 7.21$7.04(\mathrm{~m}, 4 \mathrm{H}), 3.82(\mathrm{~d}, J=12.3 \mathrm{~Hz}, 1 \mathrm{H}), 3.77(\mathrm{~d}, J=12.4 \mathrm{~Hz}$, $1 \mathrm{H}), 2.58-2.43(\mathrm{~m}, 2 \mathrm{H}), 2.41(\mathrm{~s}, 3 \mathrm{H}), 2.40-2.25(\mathrm{~m}, 2 \mathrm{H})$, 2.03-1.90 (m, 1H), $1.87-1.73(\mathrm{~m}, 1 \mathrm{H}) \mathrm{ppm} .{ }^{13} \mathrm{C}\left\{{ }^{1} \mathrm{H}\right\} \mathrm{NMR}$ $\left(101 \mathrm{MHz}, \mathrm{CDCl}_{3}, \delta\right): 219.1,136.9,136.0,133.1,127.5,127.5$, 126.1, 58.3, 55.1, 37.8, 32.9, 21.8, 18.7 ppm. HRMS (ESI): [M $+\mathrm{H}]^{+}$calcd for $\mathrm{C}_{13} \mathrm{H}_{16} \mathrm{NO}^{+}, 202.1226$; found, 202.1228 (loss of $\mathrm{N}_{2}$ ). IR (film): $2970(\mathrm{w}), 2102(\mathrm{~s}), 1737$ (m), $1490(\mathrm{w})$, $1453(\mathrm{w}), 1279(\mathrm{w}), 1163(\mathrm{w}), 1039(\mathrm{w}), 909(\mathrm{~m}), 760(\mathrm{~m})$, $726(\mathrm{~s}) \mathrm{cm}^{-1}$.

2-(Azidomethyl)-2-(3,4-dimethylphenyl)cyclopentanone $(6 \mathrm{l})$.

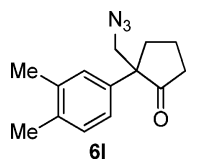


Compound $\mathbf{6 1}$ was prepared according to general method $\mathrm{E}$ using 41 ( $0.13 \mathrm{~g}, 0.50 \mathrm{mmol}, 1$ equiv), $2(265 \mathrm{mg}, 0.600 \mathrm{mmol}$, 1.20 equiv), and $\mathrm{Cu}(\mathrm{dap}){ }_{2} \mathrm{Cl}(2.2 \mathrm{mg}, 2.5 \mu \mathrm{mol}, 0.5 \mathrm{~mol} \%)$ in $\mathrm{CH}_{3} \mathrm{CN}(2.5 \mathrm{~mL}, 0.2 \mathrm{~m})$. The title compound 61 was obtained as a colorless oil $(89 \mathrm{mg}, 0.39 \mathrm{mmol}, 78 \%) . R_{f} 0.7$ (silica, pentane:EtOAc 10:1). ${ }^{1} \mathrm{H}$ NMR $\left(400 \mathrm{MHz}, \mathrm{CDCl}_{3}, \delta\right)$ : $7.20-6.94(\mathrm{~m}, 3 \mathrm{H}), 3.72(\mathrm{~d}, J=12.3 \mathrm{~Hz}, 1 \mathrm{H}), 3.46(\mathrm{~d}, J=$ $12.3 \mathrm{~Hz}, 1 \mathrm{H}), 2.70-2.54(\mathrm{~m}, 1 \mathrm{H}), 2.46-2.16(\mathrm{~m}, 9 \mathrm{H}),, 2.06-$ $1.91(\mathrm{~m}, 1 \mathrm{H}), 1.84-1.72(\mathrm{~m}, 1 \mathrm{H}) \mathrm{ppm} .{ }^{13} \mathrm{C}\left\{{ }^{1} \mathrm{H}\right\}$ NMR $(101$ $\left.\mathrm{MHz}, \mathrm{CDCl}_{3}, \delta\right): 217.6,137.2,136.2,134.0,130.1,127.8$, 123.9, 57.8, 57.4, 37.3, 31.5, 19.9, 19.3, 18.4 ppm. HRMS (ESI): $[\mathrm{M}+\mathrm{H}]^{+}$calcd. for $\mathrm{C}_{14} \mathrm{H}_{18} \mathrm{NO}^{+}, 216.1383$; found, 216.1381 (loss of $\mathrm{N}_{2}$ ). IR (film): 2972 (w), 2101 (s), 1736 (m), $1506(\mathrm{w}), 1455(\mathrm{w}), 1296(\mathrm{w}), 1159(\mathrm{w}), 910(\mathrm{~m})$, $732(\mathrm{~s}) \mathrm{cm}^{-1}$.

2-(Azidomethyl)-2-(3,4-dimethoxyphenyl)cyclopentanone $(6 \mathrm{~m})$.

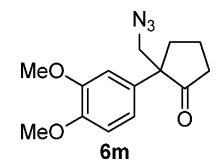

Compound $6 \mathrm{~m}$ was prepared according to general method $\mathrm{E}$ using $4 \mathrm{~m}$ (153 mg, $0.500 \mathrm{mmol}, 1.00$ equiv), 2 (265 mg, $0.600 \mathrm{mmol}, 1.2$ equiv), and $\mathrm{Cu}(\mathrm{dap})_{2} \mathrm{Cl}(2.2 \mathrm{mg}, 2.5 \mu \mathrm{mol}$, $0.5 \mathrm{~mol} \%)$ in $\mathrm{CH}_{3} \mathrm{CN}(2.5 \mathrm{~mL}, 0.2 \mathrm{~m})$. The title compound $6 \mathrm{~m}$ was obtained as a colorless oil (120 mg, $0.436 \mathrm{mmol}, 87 \%)$. $R_{f} 0.2$ (silica, pentane:EtOAc 10:1). ${ }^{1} \mathrm{H}$ NMR (400 MHz, $\left.\mathrm{CDCl}_{3}, \delta\right): 7.01-6.78(\mathrm{~m}, 3 \mathrm{H}), 3.88(\mathrm{~s}, 3 \mathrm{H}), 3.87(\mathrm{~s}, 3 \mathrm{H})$, $3.70(\mathrm{~d}, J=12.2 \mathrm{~Hz}, 1 \mathrm{H}), 3.46(\mathrm{~d}, J=12.2 \mathrm{~Hz}, 1 \mathrm{H})$, $2.63-2.53(\mathrm{~m}, 1 \mathrm{H}), 2.41-2.20(\mathrm{~m}, 3 \mathrm{H}), 2.06-1.94(\mathrm{~m}, 1 \mathrm{H})$, $1.87-1.73(\mathrm{~m}, 1 \mathrm{H})$ ppm. ${ }^{13} \mathrm{C}\left\{{ }^{1} \mathrm{H}\right\}$ NMR $\left(101 \mathrm{MHz} \mathrm{CDCl}_{3}\right.$, $\delta): 217.5,149.2,148.7,128.8,119.1,111.2,110.0,58.0$, 57.3, 56.0, 55.8, 37.4, 31.7, 18.5 ppm. HRMS (ESI): $[\mathrm{M}+$ $\mathrm{Na}]^{+}$calcd for $\mathrm{C}_{14} \mathrm{H}_{17} \mathrm{~N}_{3} \mathrm{NaO}_{3}{ }^{+}, 298.1162$; found, 298.1170. IR (film): 2971 (s), 2902 (s), 2104 (m), 1735 (w), 1519 (m), 1465 (w), $1409(\mathrm{~m}), 1259(\mathrm{~s}), 1151(\mathrm{w}), 1066(\mathrm{~s}), 1040(\mathrm{~s}), 872(\mathrm{w})$, $742(\mathrm{w}) \mathrm{cm}^{-1}$.

2-(Azidomethyl)-2-(benzo[d][1,3]dioxol-5-yl)cyclopentanone (6n).

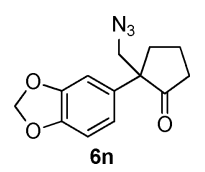

Compound 6n was prepared according to general method $\mathrm{E}$ using 4n (145 mg, $0.500 \mathrm{mmol}, 1.00$ equiv), 2 (0.26 g, $0.60 \mathrm{mmol}, 1.2$ equiv), and $\mathrm{Cu}(\text { dap })_{2} \mathrm{Cl}(2.2 \mathrm{mg}, 2.5 \mu \mathrm{mol}$, $0.5 \mathrm{~mol} \%)$ in $\mathrm{CH}_{3} \mathrm{CN}(2.5 \mathrm{~mL}, 0.2 \mathrm{~m})$. The title compound 6n was obtained as a colorless oil $(109 \mathrm{mg}, 0.420 \mathrm{mmol}$, 84\%). $\quad R_{f} 0.4$ (silica, pentane:EtOAc 10:1). ${ }^{1} \mathrm{H}$ NMR $\left(400 \mathrm{MHz}, \mathrm{CDCl}_{3}, \delta\right): 6.88(\mathrm{~d}, J=1.9 \mathrm{~Hz}, 1 \mathrm{H}), 6.81(\mathrm{dd}$, $J=8.2,1.9 \mathrm{~Hz}, 1 \mathrm{H}), 6.75(\mathrm{~d}, J=8.2 \mathrm{~Hz}, 1 \mathrm{H}), 5.92(\mathrm{q}, J=$ $1.4 \mathrm{~Hz}, 2 \mathrm{H}), 3.63(\mathrm{~d}, J=12.3 \mathrm{~Hz}, 1 \mathrm{H}), 3.41(\mathrm{~d}, J=12.3 \mathrm{~Hz}$, $1 \mathrm{H}), 2.50(\mathrm{~m}, 1 \mathrm{H}), 2.33(\mathrm{~m}, 1 \mathrm{H}), 2.27-2.16(\mathrm{~m}, 2 \mathrm{H}), 2.05-$ $1.90(\mathrm{~m}, 1 \mathrm{H}), 1.76(\mathrm{~m}, 1 \mathrm{H}) \mathrm{ppm} .{ }^{13} \mathrm{C}\left\{{ }^{1} \mathrm{H}\right\} \operatorname{NMR}(101 \mathrm{MHz}$, $\left.\mathrm{CDCl}_{3}, \delta\right): 217.1,148.2,147.0,130.2,120.1,108.3,107.1$, 101.2, 57.8, 57.2, 37.2, 31.8, 18.3 ppm. HRMS (ESI): $[\mathrm{M}+\mathrm{Na}]^{+}$calcd. for $\mathrm{C}_{13} \mathrm{H}_{13} \mathrm{~N}_{3} \mathrm{NaO}_{3}^{+}, 282.0849$; found, 282.0849. IR (film): 3454 (w), 2972 (w), 2890 (w), 2780 (w), 2102 (s), 1734 (s), 1611 (w), 1505 (s), 1488 (s), 1437 (m), $1297(\mathrm{w}), 1237$ (s), $1157(\mathrm{w}), 1115(\mathrm{w}), 1039(\mathrm{~s}), 911$ (s) $\mathrm{cm}^{-1}$.
2-(Azidomethyl)-2-(naphthalen-2-yl)cyclopentanone (6o).<smiles>NCC1(c2cccc3ccccc23)CCCC1=O</smiles>

Compound 6o was prepared according to general method $\mathrm{E}$ using 4o (148 mg, $0.500 \mathrm{mmol}, 1.00$ equiv), 2 (265 mg, $0.600 \mathrm{mmol}, 1.20$ equiv), and $\mathrm{Cu}(\mathrm{dap})_{2} \mathrm{Cl}(2.2 \mathrm{mg}, 2.5 \mu \mathrm{mol}$, $0.5 \mathrm{~mol} \%)$ in $\mathrm{CH}_{3} \mathrm{CN}(2.5 \mathrm{~mL}, 0.2 \mathrm{~m})$. The title compound 6o was obtained as a colorless oil (126 mg, $0.475 \mathrm{mmol}, 95 \%)$. $R_{f} 0.6$ (silica, pentane:EtOAc 10:1). ${ }^{1} \mathrm{H}$ NMR (400 MHz, $\left.\mathrm{CDCl}_{3}, \delta\right): 7.92-7.77(\mathrm{~m}, 4 \mathrm{H}), 7.62-7.45(\mathrm{~m}, 3 \mathrm{H}), 3.83(\mathrm{~d}$, $J=12.3 \mathrm{~Hz}, 1 \mathrm{H}), 3.58(\mathrm{~d}, J=12.3 \mathrm{~Hz}, 1 \mathrm{H}), 2.82-2.67$ (m, 1H), 2.47-2.24 (m, 3H), 2.09-1.96 (m, 1H), 1.88-1.73 (m, 1H) ppm. ${ }^{13} \mathrm{C}\left\{{ }^{1} \mathrm{H}\right\}$ NMR $\left(101 \mathrm{MHz}, \mathrm{CDCl}_{3} ; 1\right.$ aromatic signal was not resolved, $\delta$ ): 217.3, 134.0, 133.1, 132.5, 128.8, 128.0, 127.4, 126.3, 126.0, 124.1, 57.8, 57.6, 37.4, 31.7, 18.4 ppm. HRMS (ESI): $[\mathrm{M}+\mathrm{Na}]^{+}$calcd. for $\mathrm{C}_{16} \mathrm{H}_{15} \mathrm{~N}_{3} \mathrm{NaO}^{+}$, 288.1107; found, 288.1118. IR (film): 3061 (w), $2972(\mathrm{w})$, 2105 (s), 1738 (m), 1598 (w), 1507 (w), $1437(\mathrm{w}), 1343(\mathrm{w})$, $1301(\mathrm{w}), 1275(\mathrm{w}), 1160(\mathrm{w}), 1036(\mathrm{w}), 968(\mathrm{w}), 863(\mathrm{w})$, $821(\mathrm{w}) \mathrm{cm}^{-1}$.

2-(Azidomethyl)-2-(naphthalen-1-yl)cyclopentanone (6p).

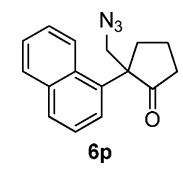

Compound 6p was prepared according to general method $\mathrm{E}$ using 4p (0.15 g, $0.50 \mathrm{mmol}, 1$ equiv), 2 ( $265 \mathrm{mg}, 0.600 \mathrm{mmol}$, 1.20 equiv), and $\mathrm{Cu}(\mathrm{dap})_{2} \mathrm{Cl}(2.2 \mathrm{mg}, 2.5 \mu \mathrm{mol}, 0.5 \mathrm{~mol} \%)$ in $\mathrm{CH}_{3} \mathrm{CN}(2.5 \mathrm{~mL}, 0.2 \mathrm{~m})$. The title compound $6 \mathrm{p}$ was obtained as a colorless oil $(105 \mathrm{mg}, 0.396 \mathrm{mmol}, 79 \%) . R_{f} 0.7$ (silica, pentane:EtOAc 10:1). ${ }^{1} \mathrm{H}$ NMR (400 $\mathrm{MHz}, \mathrm{CDCl}_{3}, \delta$ ): 8.16$8.09(\mathrm{~m}, 1 \mathrm{H}), 7.89$ (dd, $J=7.9,1.7 \mathrm{~Hz}, 1 \mathrm{H}), 7.79(\mathrm{dt}, J=8.2$, $1.1 \mathrm{~Hz}, 1 \mathrm{H}), 7.59-7.46(\mathrm{~m}, 2 \mathrm{H}), 7.35(\mathrm{dd}, J=8.2,7.4 \mathrm{~Hz}$, $1 \mathrm{H}), 7.22(\mathrm{dd}, J=7.4,1.1 \mathrm{~Hz}, 1 \mathrm{H}), 4.21(\mathrm{~d}, J=12.5 \mathrm{~Hz}, 1 \mathrm{H})$, $4.00(\mathrm{~d}, J=12.5 \mathrm{~Hz}, 1 \mathrm{H}), 2.89$ (dddd, $J=13.0,7.2,3.9$, $1.5 \mathrm{~Hz}, 1 \mathrm{H}$ ), 2.66 (ddd, $J=13.0,9.5,7.2 \mathrm{~Hz}, 1 \mathrm{H}$ ), 2.56 (dddd, $J=18.8,9.5,4.8,1.5 \mathrm{~Hz}, 1 \mathrm{H}), 2.38(\mathrm{dt}, J=18.8,8.6 \mathrm{~Hz}, 1 \mathrm{H})$, 2.08-1.92 (m), $1.82-1.68(\mathrm{~m}, 1 \mathrm{H})$ ppm. ${ }^{13} \mathrm{C}\left\{{ }^{1} \mathrm{H}\right\}$ NMR $\left(101 \mathrm{MHz}, \mathrm{CDCl}_{3}, \delta\right): 219.3,135.3,134.3,130.5,129.9,129.1$, $125.8,125.7,125.4,125.0,125.0,58.7,55.4,37.6,33.0$, 18.9 ppm. HRMS (ESI): $\left[\mathrm{M}^{+}\right]$calcd. for $\mathrm{C}_{16} \mathrm{H}_{15} \mathrm{NO}, 237.1148$; found, 237.1156 (loss of $\mathrm{N}_{2}$ ). IR (film): 2972 (m), 2902 (m), 2100 (s), 1737 (s), 1599 (w), 1510 (w), 1448 (w), 1398 (w), $1297(\mathrm{w}), 1252(\mathrm{w}), 1164(\mathrm{w}), 1077(\mathrm{~m}), 1052(\mathrm{~m}), 910(\mathrm{w})$, $803(\mathrm{~m}), 776(\mathrm{~s}), 733(\mathrm{~m}) \mathrm{cm}^{-1}$.

4-(Azidomethyl)-4-phenyldihydrofuran-3(2H)-one (6q).

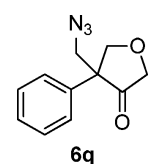

Compound $6 \mathbf{q}$ was prepared according to general method $\mathrm{E}$ using 4q (157 mg, $0.500 \mathrm{mmol}, 1.00$ equiv), $2(265 \mathrm{mg}$, $0.600 \mathrm{mmol}, 1.20$ equiv), and $\mathrm{Cu}(\text { dap })_{2} \mathrm{Cl}(2.2 \mathrm{mg}, 2.5 \mu \mathrm{mol}$, $0.5 \mathrm{~mol} \%)$ in $\mathrm{CH}_{3} \mathrm{CN}(2.5 \mathrm{~mL}, 0.2 \mathrm{~m})$. The title compound 6q was obtained as a colorless oil ( $82 \mathrm{mg}, 0.29 \mathrm{mmol}, 58 \%)$. $R_{f} 0.6$ (silica, pentane:EtOAc 10:1). ${ }^{1} \mathrm{H} \mathrm{NMR}\left(400 \mathrm{MHz}, \mathrm{CDCl}_{3}\right.$, 3:1 mixture of keto/enol tautomers, $\delta): 7.47-7.28(\mathrm{~m}, 5 \mathrm{H})$, 
5.07 (s, 1H), 4.77 (d, $J=8.4 \mathrm{~Hz}, 1 \mathrm{H}), 4.72(\mathrm{~d}, J=10.0 \mathrm{~Hz}$, $1 \mathrm{H}), 4.47$ (d, $J=10.0 \mathrm{~Hz}, 1 \mathrm{H}), 4.43(\mathrm{~d}, J=8.4 \mathrm{~Hz}, 1 \mathrm{H}), 4.11$ (d, $J=17.3 \mathrm{~Hz}, 1 \mathrm{H}), 4.03(\mathrm{~d}, J=17.4 \mathrm{~Hz}, 1 \mathrm{H}), 3.92(\mathrm{~d}, J=$ $13.9 \mathrm{~Hz}, 1 \mathrm{H}), 3.84$ (d, $J=12.5 \mathrm{~Hz}, 1 \mathrm{H}), 3.69$ (d, $J=12.5 \mathrm{~Hz}$, $1 \mathrm{H}), \quad 3.61(\mathrm{~d}, J=13.9 \mathrm{~Hz}, 1 \mathrm{H})$ ppm. ${ }^{13} \mathrm{C}\left\{{ }^{1} \mathrm{H}\right\}$ NMR $\left(101 \mathrm{MHz}, \mathrm{CDCl}_{3}\right.$, keto, $\left.\delta\right): 212.8,135.4,129.2,128.3,126.6$, 74.1, 71.1, 68.1, 54.9 ppm. HRMS (ESI): $[\mathrm{M}+\mathrm{H}]^{+}$calcd. for $\mathrm{C}_{11} \mathrm{H}_{12} \mathrm{NO}_{2}{ }^{+}, 190.0863$; found, 190.0863 (loss of $\mathrm{N}_{2}$ ). IR (film): 2968 (w), 2879 (w), 2105 (s), 1760 (m), 1499 (w), $1448(\mathrm{w}), 1299(\mathrm{w}), 1190(\mathrm{w}), 1067(\mathrm{w}), 967(\mathrm{w}), 930(\mathrm{w})$, $764(\mathrm{w}) \mathrm{cm}^{-1}$.

2-(Azidomethyl)-2-(4-(trifluoromethyl)phenyl)-1-oxaspiro[2.3]hexane (7t).

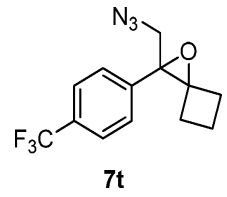

Compound $7 \mathbf{t}$ was prepared according to general method $\mathrm{E}$ using 4t (124 mg, $0.500 \mathrm{mmol}, 1.00$ equiv), $2(265 \mathrm{mg}$, $0.600 \mathrm{mmol}, 1.20$ equiv), and $\mathrm{Cu}(\mathrm{dap})_{2} \mathrm{Cl}(2.2 \mathrm{mg}, 2.5 \mu \mathrm{mol}$, $0.50 \mathrm{~mol} \%)$ in $\mathrm{CH}_{3} \mathrm{CN}(2.5 \mathrm{~mL}, 0.20 \mathrm{~m})$. The title compound $7 \mathbf{t}$ was obtained as a colorless oil $(72 \mathrm{mg}, 0.33 \mathrm{mmol}, 66 \%) . R_{f}$ 0.5 (silica, pentane:EtOAc 10:1). ${ }^{1} \mathrm{H}$ NMR $\left(400 \mathrm{MHz} \mathrm{CDCl}_{3}\right.$, $\delta): 7.64(\mathrm{~d}, J=8.2 \mathrm{~Hz}, 2 \mathrm{H}), 7.46(\mathrm{~d}, J=8.2 \mathrm{~Hz}, 2 \mathrm{H}), 3.89$ $(\mathrm{d}, J=13.4 \mathrm{~Hz}, 1 \mathrm{H}), 3.51(\mathrm{~d}, J=13.4 \mathrm{~Hz}, 1 \mathrm{H}), 2.72-2.57$ (m, $1 \mathrm{H}), 2.57-2.44(\mathrm{~m}, 1 \mathrm{H}), 2.42-2.26(\mathrm{~m}, 1 \mathrm{H}), 2.01-1.88$ (m, 1H), 1.87-1.68 (m, 2H) ppm. ${ }^{13} \mathrm{C}\left\{{ }^{1} \mathrm{H}\right\}$ NMR (101 MHz, $\left.\mathrm{CDCl}_{3}, \delta\right): 140.2,130.1(\mathrm{q}, J=32.5 \mathrm{~Hz}), 126.8,125.4(\mathrm{q}, J=$ $3.8 \mathrm{~Hz}), 124.0(\mathrm{q}, J=272.9 \mathrm{~Hz}), 69.8,65.0,54.1,29.4,29.0$, 12.7 ppm. ${ }^{19} \mathrm{~F}$ NMR (376 $\left.\mathrm{MHz}, \mathrm{CDCl}_{3}, \delta\right):-62.6$ (s) ppm. HRMS (ESI): $[\mathrm{M}+\mathrm{H}]^{+}$calcd. for $\mathrm{C}_{13} \mathrm{H}_{13} \mathrm{~F}_{3} \mathrm{NO}^{+}, 256.0944$; found, 256.0945 (loss of $\mathrm{N}_{2}$ ). IR (film): 2987 (s), 2972 (s), $2902(\mathrm{~m}), 2105(\mathrm{~m}), 1408(\mathrm{~m}), 1329(\mathrm{~s}), 1251(\mathrm{w})$, $1168(\mathrm{~m}), 1127(\mathrm{~m}), 1067(\mathrm{~s}), 894(\mathrm{w}), 846(\mathrm{w}) \mathrm{cm}^{-1}$.

Product Functionalization. 2-Phenyl-2-((4-phenyl-1H-1,2,3-triazol-1-yl)methyl)cyclopentanone (27). Triethylamine $(0.10 \mathrm{~mL}$, $0.70 \mathrm{mmol}, 2$ equiv) followed by phenylacetylene $(77 \mu \mathrm{L}$, $0.70 \mathrm{mmol}, 2$ equiv) were added to a solution of $6 \mathrm{a}(75 \mathrm{mg}$, $0.35 \mathrm{mmol}, 1$ equiv) and $\mathrm{CuI}(6 \mathrm{mg}, 0.04 \mathrm{mmol}, 10 \mathrm{~mol} \%)$ in THF $(1.7 \mathrm{~mL}, 0.2 \mathrm{~m})$ at room temperature, and the resulting mixture was stirred for $16 \mathrm{~h}$ at room temperature. Thereafter, the mixture was concentrated, and the residue was purified by column chromatography (silica, pentane:EtOAc 20:1 to 10:1), affording $102 \mathrm{mg}$ $(0.321 \mathrm{mmol}, 92 \%)$ of the title compound 27 as a colorless oil. $R_{f} 0.3$ (silica, pentane:EtOAc 10:1). ${ }^{1} \mathrm{H}$ NMR $\left(400 \mathrm{MHz}, \mathrm{CDCl}_{3}, \delta\right): 7.61-$ $7.55(\mathrm{~m}, 2 \mathrm{H}), 7.29-7.10(\mathrm{~m}, 9 \mathrm{H}), 4.60(\mathrm{~d}, J=14.1 \mathrm{~Hz}, 1 \mathrm{H}), 4.49$ $(\mathrm{d}, J=14.1 \mathrm{~Hz}, 1 \mathrm{H}), 2.55-2.44(\mathrm{~m}, 1 \mathrm{H}), 2.25-2.13(\mathrm{~m}, 1 \mathrm{H}), 1.99$ $(\mathrm{dt}, J=19.5,9.2 \mathrm{~Hz}, 1 \mathrm{H}), 1.91-1.82(\mathrm{~m}, 1 \mathrm{H}), 1.72-1.63(\mathrm{~m}, 1 \mathrm{H})$, 1.62-1.49 (m, 1H) ppm. ${ }^{13} \mathrm{C}\left\{{ }^{1} \mathrm{H}\right\}$ NMR $\left(101 \mathrm{MHz}, \mathrm{CDCl}_{3}, \delta\right)$ : 217.2, 147.3, 135.7, 130.3, 129.1, 128.6, 128.1, 128.0, 126.6, 125.5, 120.7, 58.6, 56.0, 37.0, 31.4, 17.9 ppm. HRMS (ESI): $[\mathrm{M}+\mathrm{Na}]^{+}$ calcd. for $\mathrm{C}_{20} \mathrm{H}_{19} \mathrm{~N}_{3} \mathrm{NaO}^{+}, 340.1420$; found, 340.1420. IR (film): $3147(w), 3066(w), 2977(w), 2944(w), 2888(w), 2250(w)$, $1734(\mathrm{w}), 1464(\mathrm{w}), 1356(\mathrm{w}), 1231(\mathrm{w}), 1158(\mathrm{w}), 1077(\mathrm{w})$, $1048(\mathrm{w}), 907(\mathrm{~s}), 766(\mathrm{~m}), 728(\mathrm{~s}) \mathrm{cm}^{-1}$.

Benzyl ((2-oxo-1-Phenylcyclopentyl)methyl)carbamate (28). A mixture of $6 \mathrm{a}(75 \mathrm{mg}, 0.35 \mathrm{mmol}, 1$ equiv) and $\mathrm{Pd} / \mathrm{C}(7 \mathrm{mg}$, $\left.10 \%_{\mathrm{w} / \mathrm{w}}, 10 \% \mathrm{Pd}\right)$ in methanol $(1.7 \mathrm{~mL}, 0.2 \mathrm{~m})$ was purged with hydrogen and then stirred for $16 \mathrm{~h}$ under a hydrogen atmosphere. Thereafter, the mixture was filtered through a plug of Celite and concentrated. The crude amine was dissolved in $\mathrm{CH}_{2} \mathrm{Cl}_{2}(0.7 \mathrm{~mL}, 0.5$ $\mathrm{m})$, then $\mathrm{NEt}_{3}(0.10 \mathrm{~mL}, 0.70 \mathrm{mmol}, 2$ equiv) followed by $\mathrm{CbzCl}$ ( $75 \mu \mathrm{L}, 0.52 \mathrm{mmol}, 1.5$ equiv) were added, and stirring was continued for $3 \mathrm{~h}$ at room temperature. Then, the reaction was quenched with water $(5 \mathrm{~mL})$ and extracted with $\mathrm{CH}_{2} \mathrm{Cl}_{2}(3 \times 10 \mathrm{~mL})$. The combined organic extracts were washed with brine and dried over $\mathrm{MgSO}_{4}$. The drying agent was filtered off, and the solvent was evaporated. The residue was purified by column chromatography (silica, pentane: EtOAc 10:1) affording $74 \mathrm{mg}(0.23 \mathrm{mmol}, 66 \%)$ of the title compound 28 as a colorless oil. $R_{f} 0.5$ (silica, pentane:EtOAc 10:1). ${ }^{1} \mathrm{H}$ NMR $\left(400 \mathrm{MHz}, \mathrm{CDCl}_{3}, \delta\right): 7.25-7.14(\mathrm{~m}, 10 \mathrm{H}), 4.94(\mathrm{~s}, 2 \mathrm{H}), 3.49$ (dd, $J=14.0,6.7 \mathrm{~Hz}, 1 \mathrm{H}), 3.40(\mathrm{dd}, J=14.0,6.7 \mathrm{~Hz}, 1 \mathrm{H}), 2.46-2.36$ $(\mathrm{m}, 1 \mathrm{H}), 2.30-1.99(\mathrm{~m}, 3 \mathrm{H}), 1.90-1.79(\mathrm{~m}, 1 \mathrm{H}), 1.74-1.60$ (m, 1H) ppm. ${ }^{13} \mathrm{C}\left\{{ }^{1} \mathrm{H}\right\}$ NMR $\left(101 \mathrm{MHz}, \mathrm{CDCl}_{3}, \delta\right): 219.5,156.5$, 137.6, 136.3, 128.8, 128.4, 128.0, 127.9, 127.4, 126.7, 66.6, 58.3, 47.0, $37.7,32.5,18.3$ ppm. HRMS (ESI): $[\mathrm{M}+\mathrm{Na}]^{+}$calcd. for $\mathrm{C}_{20} \mathrm{H}_{21} \mathrm{NNaO}_{3}{ }^{+}$, 346.1414; found, 346.1420. IR (film): 3440 (w), 3350 (w), 3029 (w), 2965 (w), $2886(w), 2250(w), 1721(s)$, $1509(\mathrm{~m}), 1456(\mathrm{w}), 1404(\mathrm{w}), 1232(\mathrm{~s}), 1137(\mathrm{w}), 1010(\mathrm{w})$, $911(\mathrm{~m}), 733(\mathrm{~s}) \mathrm{cm}^{-1}$.

2-(Isothiocyanatomethyl)-2-phenylcyclopentanone (29). Triphenylphosphine $(0.11 \mathrm{~g}, 0.42 \mathrm{mmol}, 1.2$ equiv) was added at room temperature to a solution of $6 \mathrm{a}\left(75 \mathrm{mg}, 0.35 \mathrm{mmol}, 1\right.$ equiv) and $\mathrm{CS}_{2}$ $(0.30 \mathrm{~mL}, 5.0 \mathrm{mmol}, 14$ equiv) in THF $(0.7 \mathrm{~mL}, 0.5 \mathrm{~m})$, and the resulting mixture was stirred for $3 \mathrm{~h}$ at room temperature. Thereafter, the mixture was concentrated, and the residue was purified by column chromatography (silica, pentane:EtOAc 50:1) affording $58 \mathrm{mg}$ $(0.25 \mathrm{mmol}, 72 \%)$ of the title compound 29 as a colorless oil. $R_{f}$ 0.3 (silica, pentane:EtOAc 20:1). ${ }^{1} \mathrm{H}$ NMR $\left(400 \mathrm{MHz} \mathrm{CDCl}_{3}, \delta\right)$ : 7.49-7.28 (m, 5H), $3.85(\mathrm{~d}, J=14.2 \mathrm{~Hz}, 1 \mathrm{H}), 3.70(\mathrm{~d}, J=14.2 \mathrm{~Hz}$, $1 \mathrm{H}), 2.84-2.67(\mathrm{~m}, 1 \mathrm{H}), 2.45-2.20(\mathrm{~m}, 3 \mathrm{H}), 2.09-1.98(\mathrm{~m}, 1 \mathrm{H})$, $1.88-1.73(\mathrm{~m}, 1 \mathrm{H}) \mathrm{ppm} .{ }^{13} \mathrm{C}\left\{{ }^{1} \mathrm{H}\right\}$ NMR $\left(101 \mathrm{MHz}, \mathrm{CDCl}_{3}, \delta\right)$ : 215.9, 135.4, 131.9, 129.1, 128.1, 126.6, 57.8, 51.6, 37.0, 31.9, 18.3 ppm. HRMS (ESI): $[\mathrm{M}+\mathrm{H}]^{+}$calcd for $\mathrm{C}_{13} \mathrm{H}_{14} \mathrm{NOS}^{+}, 232.0791$; found, 232.0801. IR (film): 2972 (s), 2901 (s), 2188 (m), 2084 (s), 1736 (s), 1599 (w), $1497(\mathrm{w}), 1447(\mathrm{~m}), 1404(\mathrm{~m}), 1339(\mathrm{~m})$, $1158(\mathrm{~m}), 1067(\mathrm{~s}), 880(\mathrm{w}), 759(\mathrm{~m}) \mathrm{cm}^{-1}$.

Established Azidation Reactions Performed with tBuABX (1d) and ABZ (2). 4-Phenylpent-4-enoic Acid (8). Following a reported procedure, ${ }^{11}$ a solution of $t \mathrm{BuOK}(4.91 \mathrm{~g}, 43.8 \mathrm{mmol}$, 2.6 equiv) in dry THF $(0.5 \mathrm{M})$ was added under nitrogen to bromo(methyl)triphenylphosphorane (7.82 g, $21.9 \mathrm{mmol}, 1.3$ equiv) in portions at $0{ }^{\circ} \mathrm{C}$. The mixture was stirred at $0{ }^{\circ} \mathrm{C}$ for $30 \mathrm{~min}$, and a solution of 4-oxo-4-phenylbutanoic acid (30) (3.00 g, $16.8 \mathrm{mmol}$, 1.0 equiv) in dry THF $(1 \mathrm{M})$ was added dropwise. The reaction was stirred at $0{ }^{\circ} \mathrm{C}$ for $1 \mathrm{~h}$ and at room temperature overnight. The solvent was removed in vacuo, and the residue was diluted with DCM and aqueous $\mathrm{NaOH}(1 \mathrm{M})$. The aqueous layer was separated, washed with DCM, and acidified to $\mathrm{pH} 1$ with concentrated $\mathrm{HCl}(35 \%)$. DCM was added, and the organic compound was extracted twice with DCM. The organic layer was washed with water, dried over $\mathrm{MgSO}_{4}$, and concentrated in vacuo. The crude product was purified by flash column chromatography $\left(\mathrm{SiO}_{2}, \mathrm{DCM} / \mathrm{MeOH}\right.$ : $100 / 0$ then $95 / 5$ and 90/10) to give 4-phenylpent-4-enoic acid (8) (2.38 g, $13.5 \mathrm{mmol}$, $80 \%$ ). $R_{f}$ (silica, DCM/MeOH 9/1) 0.70. ${ }^{1} \mathrm{H}$ NMR (400 MHz, $\left.\mathrm{CDCl}_{3}, \delta\right): 11.60$ (bs, $\left.1 \mathrm{H}\right), 7.46-7.27(\mathrm{~m}, 5 \mathrm{H}), 5.35(\mathrm{~s}, 2 \mathrm{H}), 2.87$ $(\mathrm{t}, J=7.8 \mathrm{~Hz}, 2 \mathrm{H}), 2.56(\mathrm{dd}, J=8.9,6.7 \mathrm{~Hz}, 2 \mathrm{H}) \mathrm{ppm}$. The signals of the ${ }^{1} \mathrm{H}$ NMR spectra were in accordance with the data reported in the literature. ${ }^{11}$

$N$-Phenyl- $N$-((2-vinylphenyl)sulfonyl)methacrylamide (10). Following a reported procedure, ${ }^{42}$ 2-bromobenzene-1-sulfonyl chloride (31) (1.34 g, $5.24 \mathrm{mmol}, 1.0$ equiv) was dissolved in DMF ( $52 \mathrm{~mL})$. The resulting colorless solution was cooled to $0{ }^{\circ} \mathrm{C}$ (ice-water bath), and aniline (32) (1.9 mL, $21 \mathrm{mmol}, 4.0$ equiv) was added dropwise via syringe. The cooling bath was removed, and the mixture was stirred at room temperature for $1.5 \mathrm{~h}$. During this time, it became pale yellow. The reaction was then quenched by the addition of water $(50 \mathrm{~mL})$. The aqueous layer was extracted with EtOAc $(4 \times 50 \mathrm{~mL})$. The organic extracts were washed with aq. $\mathrm{HCl}(3 \times 30 \mathrm{~mL})$ and twice with brine, dried over $\mathrm{MgSO}_{4}$, filtered, and concentrated under vacuum. The resulting yellow, crude oil was submitted to flash column chromatography $\left(\mathrm{SiO}_{2}\right.$; pentane/EtOAc in pentane $19 / 1$ to $\left.6 / 4\right)$ to provide 2-bromo- $\mathrm{N}$ phenylbenzenesulfonamide (33) (0.819 g, $2.62 \mathrm{mmol}, 50 \%)$ as a pale yellow, crystalline solid. $R_{f}$ (silica, pentane/EtOAc 4/1) $0.39 .{ }^{1} \mathrm{H}$ NMR 
$\left(400 \mathrm{MHz}, \mathrm{CDCl}_{3}, \delta\right): 8.02(\mathrm{~m}, 1 \mathrm{H}), 7.70(\mathrm{~m}, 1 \mathrm{H}), 7.36(\mathrm{~m}, 2 \mathrm{H})$, $7.25-7.18(\mathrm{~m}, 2 \mathrm{H}), 7.18-7.06(\mathrm{~m}, 4 \mathrm{H}) \mathrm{ppm}$. The signals of the ${ }^{1} \mathrm{H}$ NMR spectra were in accordance with the data reported in the literature. ${ }^{43}$

Following a reported procedure, ${ }^{44}$ a $25 \mathrm{~mL}$ round-bottom test tube was charged with 2-bromo- $N$-phenylbenzenesulfonamide (33) $(0.600 \mathrm{~g}, 1.92 \mathrm{mmol}, 1.0$ equiv $), \mathrm{PdCl}_{2}\left(\mathrm{PPh}_{3}\right)_{2}(67 \mathrm{mg}$, $0.096 \mathrm{mmol}, 5 \mathrm{~mol} \%)$, potassium (vinyl)trifluoroborate $(0.283 \mathrm{~g}$, $2.11 \mathrm{mmol}, 1.1$ equiv), and cesium carbonate $(1.88 \mathrm{~g}, 5.77 \mathrm{mmol}$, 3.0 equiv). The tube was sealed, evacuated, and backfilled with nitrogen (3 times). A 9:1 mixture of THF and water (degassed; $9.0 \mathrm{~mL}$ ) was then added via syringe. The resulting mixture was then heated at $85-90{ }^{\circ} \mathrm{C}$ under stirring, turning from a clear, pale brown solution into a dark brown suspension. After $16 \mathrm{~h}$, the mixture was allowed to cool down to room temperature, and it was diluted with water $(15 \mathrm{~mL})$. The aqueous layer was then extracted with diethyl ether $(3 \times 20 \mathrm{~mL})$. The combined organic extracts were washed with brine, dried over $\mathrm{MgSO}_{4}$, filtered, and concentrated under vacuum to furnish an orange crude oil. The latter was submitted to column chromatography (silica; pentane/EtOAc in pentane 19:1 to 6:4) to provide pure $\mathrm{N}$-phenyl-2-vinylbenzenesulfonamide (34) (0.425 g, $1.64 \mathrm{mmol}, 85 \%)$ as a pale yellow solid. $R_{f}$ (pentane:EtOAc, 4:1) 0.4 . ${ }^{1} \mathrm{H}$ NMR $\left(400 \mathrm{MHz}, \mathrm{CDCl}_{3}, \delta\right): 7.89(\mathrm{dd}, J=7.9,1.3 \mathrm{~Hz}, 1 \mathrm{H}), 7.61$ $(\mathrm{dd}, J=17.3,11.0 \mathrm{~Hz}, 1 \mathrm{H}), 7.56-7.45(\mathrm{~m}, 2 \mathrm{H}), 7.30(\mathrm{td}, J=7.6$, $1.6 \mathrm{~Hz}, 1 \mathrm{H}), 7.17(\mathrm{t}, J=7.8 \mathrm{~Hz}, 2 \mathrm{H}), 7.06(\mathrm{~m}, 1 \mathrm{H}), 7.00-6.94$ $(\mathrm{m}, 2 \mathrm{H}, \mathrm{ArH}), 6.52(\mathrm{br} \mathrm{s}, 1 \mathrm{H}), 5.70(\mathrm{dd}, J=17.3,1.3 \mathrm{~Hz}, 1 \mathrm{H}), 5.56$ (dd, $J=11.0,1.3 \mathrm{~Hz}, 1 \mathrm{H}) \mathrm{ppm}$.

Following a reported procedure, ${ }^{45}$ in a $50 \mathrm{~mL}$ two-neck, roundbottom flask, $\mathrm{N}$-phenyl-2-vinylbenzenesulfonamide (34) $(0.420 \mathrm{~g}$, $1.62 \mathrm{mmol}, 1.0$ equiv) was dissolved in DCM (dry; $8.1 \mathrm{~mL})$, and the resulting solution was cooled to $0{ }^{\circ} \mathrm{C}$ (ice-water bath). Triethylamine (0.68 mL, $4.9 \mathrm{mmol}, 3.0$ equiv) and methacryloyl chloride $(0.34 \mathrm{~mL}$, $3.2 \mathrm{mmol}, 2.0$ equiv) were added by syringe in this order. The cooling bath was removed, and the mixture was stirred at room temperature for $20 \mathrm{~h}$. The reaction was then quenched with sat. aq. $\mathrm{NaHCO}_{3}$ $(10 \mathrm{~mL})$. The aqueous layer was extracted with DCM $(4 \times 15 \mathrm{~mL})$, and the combined organic extracts were washed with brine, dried over $\mathrm{MgSO}_{4}$, filtered, and concentrated under vacuum. The resulting pale orange crude oil was submitted to column chromatography $\left(\mathrm{SiO}_{2}\right.$; pentane:EtOAc in pentane 19:1 to 6:4) to afford pure $N$-phenyl- $N$ ((2-vinylphenyl)sulfonyl)methacrylamide (10) (0.454 g, $1.39 \mathrm{mmol}$, $86 \%)$ as a pale yellow solid. $R_{f}$ (silica, pentane:EtOAc, 4:1) 0.45 . ${ }^{1} \mathrm{H}$ NMR $\left(400 \mathrm{MHz}, \mathrm{CDCl}_{3}, \delta\right): 8.18(\mathrm{dd}, J=8.1,1.2 \mathrm{~Hz}, 1 \mathrm{H}), 7.64-$ $7.57(\mathrm{~m}, 2 \mathrm{H}), 7.45(\mathrm{ddd}, J=8.7,6.7,2.3 \mathrm{~Hz}, 1 \mathrm{H}), 7.42-7.32$ $(\mathrm{m}, 3 \mathrm{H}), 7.29(\mathrm{dd}, J=7.7,2.0 \mathrm{~Hz}, 2 \mathrm{H}), 5.65(\mathrm{dd}, J=17.3,1.2 \mathrm{~Hz}$, $1 \mathrm{H}), 5.31(\mathrm{dd}, J=10.9,1.2 \mathrm{~Hz}, 1 \mathrm{H}), 5.31-5.29(\mathrm{~m}, 1 \mathrm{H}), 5.21(\mathrm{~d}, J=$ $1.9 \mathrm{~Hz}, 1 \mathrm{H}), 1.66(\mathrm{t}, J=1.3 \mathrm{~Hz}, 3 \mathrm{H}) \mathrm{ppm} .{ }^{13} \mathrm{C}\left\{{ }^{1} \mathrm{H}\right\} \mathrm{NMR}(101 \mathrm{MHz}$, $\left.\mathrm{CDCl}_{3}, \delta\right): 170.9,139.3,138.0,136.1,136.0,134.0,133.9,132.0$, $130.5,129.4,129.2,127.9,127.6,123.7,118.5,19.2 \mathrm{ppm}$. The signals of the ${ }^{1} \mathrm{H}$ NMR spectra were in accordance with the data reported in the literature. ${ }^{8 \mathrm{c}}$

2-Allyl-N-methoxybenzamide (16). Following a reported procedure, ${ }^{46}$ methyl 2-iodobenzoate (35) (1.21 g, $4.62 \mathrm{mmol}, 1.0$ equiv), palladium tetrakis $(0.294 \mathrm{~g}, 0.254 \mathrm{mmol}, 5.5 \mathrm{~mol} \mathrm{\%})$, and allyltributylstannane $(1.7 \mathrm{~mL}, 5.5 \mathrm{mmol}, 1.2$ equiv) were dissolved in DMF (dry; $5.1 \mathrm{~mL}$ ). The mixture was submitted to microwave irradiation in a sealed reaction vessel at $120{ }^{\circ} \mathrm{C}$ (temperature was monitored using an external surface sensor) for $10 \mathrm{~min}$, turning from yellow to dark brown. The suspension was filtered through a pad of Celite, which was then washed with several portions of EtOAc (overal, $30 \mathrm{~mL}$ ). The filtrate was washed with aq. $\mathrm{HCl}(1.0 \mathrm{M}$; $30 \mathrm{~mL}$ ), water, and brine, and it was dried over $\mathrm{MgSO}_{4}$, filtered, and concentrated under vacuum. The resulting dark brown crude product was submitted to column chromatography (silica; pentane:DCM $92: 8$ to $60: 40)$ to furnish pure methyl 2-allylbenzoate (36) $(0.650 \mathrm{~g}$, $3.69 \mathrm{mmol}, 80 \%)$ as a pale yellow oil. $R_{f}$ (silica, pentane:DCM 4:1) 0.59. ${ }^{1} \mathrm{H}$ NMR $\left(400 \mathrm{MHz}, \mathrm{CDCl}_{3}, \delta\right): 7.88(\mathrm{dd}, J=8.3,1.5 \mathrm{~Hz}, 1 \mathrm{H})$, $7.44(\mathrm{td}, J=7.5,1.5 \mathrm{~Hz}, 1 \mathrm{H}), 7.27(\mathrm{~m}, 2 \mathrm{H}), 6.01(\mathrm{ddt}, J=16.8$, 10.3, $6.4 \mathrm{~Hz}, 1 \mathrm{H}), 5.04(\mathrm{dt}, J=4.3,1.7 \mathrm{~Hz}, 1 \mathrm{H}), 5.01(\mathrm{~m}, 1 \mathrm{H}), 3.89$ $(\mathrm{s}, 3 \mathrm{H}), 3.76(\mathrm{dt}, J=6.4,1.6 \mathrm{~Hz}, 2 \mathrm{H}) \mathrm{ppm}$. The signals of the
${ }^{1} \mathrm{H}$ NMR spectra were in accordance with the data reported in the literature. $^{46}$

Following a reported procedure, ${ }^{47}$ methyl 2-allylbenzoate (36) $(0.650 \mathrm{~g}, 3.69 \mathrm{mmol}, 1.0$ equiv) was dissolved in a mixture of THF $(12.3 \mathrm{~mL}), \mathrm{MeOH}(3.1 \mathrm{~mL})$, and water $(3.1 \mathrm{~mL}) . \mathrm{LiOH}(0.883 \mathrm{~g}$, $36.9 \mathrm{mmol}, 10$ equiv) was added, and the resulting suspension was vigorously stirred overnight at room temperature. After $16 \mathrm{~h}$, the mixture was diluted with water $(30 \mathrm{~mL})$, and most of the volatile solvents were removed by evaporation under reduced pressure. The aqueous residue was washed with diethyl ether $(3 \times 20 \mathrm{~mL})$ and acidified by cautious addition of conc. $\mathrm{HCl}(32 \% \mathrm{v} / \mathrm{v})$ until $\mathrm{pH}<3$. It was then extracted with DCM $(3 \times 30 \mathrm{~mL})$. The combined organic extracts were dried over $\mathrm{MgSO}_{4}$, filtered, and concentrated under vacuum to afford 2-allylbenzoic acid (37) (0.500 g, $3.08 \mathrm{mmol}, 83 \%$ ) as a colorless solid, which did not require any further purification. ${ }^{1} \mathrm{H}$ NMR (400 MHz, $d_{6}$-DMSO; the signal corresponding to the $\mathrm{CO}_{2} \mathrm{H}$ was not resolved, $\left.\delta\right): 7.80(\mathrm{dd}, J=7.7,1.5 \mathrm{~Hz}, 1 \mathrm{H}), 7.48(\mathrm{td}$ $J=7.5,1.5 \mathrm{~Hz}, 1 \mathrm{H}), 7.35-7.26(\mathrm{~m}, 2 \mathrm{H}), 5.96(\mathrm{ddt}, J=18.5,9.4$, $6.5 \mathrm{~Hz}, 1 \mathrm{H}), 5.01(\mathrm{dd}, J=3.7,1.8 \mathrm{~Hz}, 1 \mathrm{H}), 4.97(\mathrm{~d}, J=1.6 \mathrm{~Hz}, 1 \mathrm{H})$, $3.71(\mathrm{~d}, J=6.6 \mathrm{~Hz}, 2 \mathrm{H}) \mathrm{ppm} .{ }^{13} \mathrm{C}\left\{{ }^{1} \mathrm{H}\right\} \mathrm{NMR}\left(101 \mathrm{MHz}, \mathrm{CDCl}_{3}, \delta\right)$ : 173.6, 142.8, 137.3, 133.1, 131.7, 131.1, 128.2, 126.3, 115.7, 38.6. The signals of the NMR spectra were in accordance with the data reported in the literature. ${ }^{11}$

Following a reported procedure, ${ }^{10 \mathrm{~h}}$ a $50 \mathrm{~mL}$ round-bottom test tube was charged 2-allylbenzoic acid (37) (0.400 g, $2.47 \mathrm{mmol}$, 1.0 equiv) and DCM (dry; $12.3 \mathrm{~mL}$ ). O-Methylhydroxylamine hydrochloride (0.309 g, $3.70 \mathrm{mmol}, 1.5$ equiv), DMAP (0.603 g, $4.93 \mathrm{mmol}, 2.0$ equiv), and EDCI (0.946 g, $4.93 \mathrm{mmol}, 2.0$ equiv) were added to provide a pale yellow-pink solution, which was stirred at room temperature for $20 \mathrm{~h}$. The organic solution was then treated with aq. $\mathrm{HCl}(1.0 \mathrm{M} ; 15 \mathrm{~mL})$. The aqueous layer was extracted with DCM $(3 \times 15 \mathrm{~mL})$. The combined organic extracts were washed with brine, dried over $\mathrm{MgSO}_{4}$, filtered, and concentrated under vacuum to furnish a pale yellow crude solid. The latter was submitted to column chromatography $\left(\mathrm{SiO}_{2}\right.$; pentane:EtOAc 50:50 to 30:70) to afford pure 2-allyl- $N$-methoxybenzamide (16) (0.380 g, $2.00 \mathrm{mmol}, 81 \%)$ as a colorless solid. $R_{f}$ (silica, pentane:EtOAc 5:4) $0.37 .{ }^{1} \mathrm{H}$ NMR $\left(400 \mathrm{MHz}, \mathrm{CDCl}_{3}, \delta\right): 8.42$ (br s, $\left.1 \mathrm{H}\right), 7.37-7.29(\mathrm{~m}, 2 \mathrm{H}), 7.30-$ $7.22(\mathrm{~m}, 2 \mathrm{H}), 6.00(\mathrm{ddt}, J=16.6,10.1,6.3 \mathrm{~Hz}, 1 \mathrm{H}), 5.09$ (dd, $J=$ $10.2,1.6 \mathrm{~Hz}, 1 \mathrm{H}), 5.02(\mathrm{dd}, J=17.1,1.8 \mathrm{~Hz}, 1 \mathrm{H}), 3.88(\mathrm{~s}, 3 \mathrm{H}), 3.57$ $(\mathrm{d}, J=6.3 \mathrm{~Hz}, 2 \mathrm{H}) \mathrm{ppm}$. The signals of the ${ }^{1} \mathrm{H}$ NMR spectra were in accordance with the data reported in the literature. ${ }^{48}$

$\mathrm{Cu}(\mathrm{I})$-Catalyzed Intramolecular Oxyazidation of Alkenes: Synthesis of 5-(Azidomethyl)-5-phenyldihydrofuran-2(3H)one (9). Experiment with ABX (General Method F). Following a slightly modified procedure, ${ }^{11}$ distilled dry acetonitrile (preliminarily purged with Ar for $5 \mathrm{~min} ; 1.0 \mathrm{~mL}$ ) was added in a flame-dried $4 \mathrm{~mL}$ round bottom test tube containing 4-phenylpent-4-enoic acid (8) (35.0 mg, $0.200 \mathrm{mmol}, 1.0$ equiv), ABX (1a) $(116 \mathrm{mg}, 0.400 \mathrm{mmol}$, 2.0 equiv), and $\mathrm{Cu}(\mathrm{dap})_{2} \mathrm{Cl}(0.90 \mathrm{mg}, 1.0 \mu \mathrm{mol}, 0.50 \mathrm{~mol} \%)$. The resulting solution was irradiated with blue light LEDs under stirring at room temperature for $18 \mathrm{~h}$. The reaction mixture was then concentrated, diluted with $\mathrm{Et}_{2} \mathrm{O}(10 \mathrm{~mL})$, filtered through a plug of Celite, and again concentrated under reduced pressure. The residue was submitted to flash column chromatography $\left(\mathrm{SiO}_{2}\right.$, previously deactivated with $\mathrm{Et}_{3} \mathrm{~N}$; pentane:EtOAc 90:10 to 80:20) to afford pure 5-(azidomethyl)-5-phenyldihydrofuran-2 $(3 H)$-one $(9) \quad(33.0 \mathrm{mg}$, $0.152 \mathrm{mmol}, 76 \%$ ) as a yellow solid. $R_{f}$ (silica, pentane:EtOAc 8:2) 0.45. ${ }^{1} \mathrm{H}$ NMR (400 MHz, $\left.\mathrm{CDCl}_{3}, \delta\right): 7.44-7.32$ (m, $\left.5 \mathrm{H}\right), 3.68$ $(\mathrm{d}, J=13.2 \mathrm{~Hz}, 1 \mathrm{H}), 3.53(\mathrm{~d}, J=13.2 \mathrm{~Hz}, 1 \mathrm{H}), 2.81-2.64(\mathrm{~m}, 2 \mathrm{H})$, 2.57-2.38 (m, $2 \mathrm{H}) \mathrm{ppm}$. The signals of the ${ }^{1} \mathrm{H}$ NMR spectra were in accordance with the data reported in the literature. ${ }^{11}$

Experiment with $t B u A B X$ (1d). The reaction was performed following the general method $\mathrm{F}$, starting from the same amounts of 4-phenylpent-4-enoic acid (8) and $\mathrm{Cu}(\mathrm{dap})_{2} \mathrm{Cl}$, but using $t \mathrm{BuABX}$ (1d) $(138 \mathrm{mg}, 0.400 \mathrm{mmol}, 2.0$ equiv) instead of ABX (1a). 5-(Azidomethyl)-5-phenyldihydrofuran-2(3H)-one (9) was obtained in $56 \%$ yield $(24.0 \mathrm{mg}, 0.112 \mathrm{mmol}){ }^{26}$

Experiment with $A B Z$ (2). The reaction was performed following the general method F, starting from the same amounts of 
4-phenylpent-4-enoic acid (8) and $\mathrm{Cu}(\mathrm{dap})_{2} \mathrm{Cl}$, but using $\mathrm{ABZ}$ (2) (177 mg, $0.400 \mathrm{mmol}, 2.0$ equiv) instead of ABX (1a). 5-(Azidomethyl)-5-phenyldihydrofuran-2(3H)-one (9) was obtained in $81 \%$ (35.0 mg, $0.162 \mathrm{mmol}){ }^{26}$

Generation of Functionalized Indanes through a Complex Radical Cascade Reaction: Synthesis of 3-(Azidomethyl)-1methyl- $N$-phenyl-2,3-dihydro-1 $H$-indene-1-carboxamide (11). Experiment with $t B u A B X(1 d)$. Following a reported procedure, ${ }^{8 \mathrm{C}}$ a $25 \mathrm{~mL}$ round-bottom test tube was charged with $\mathrm{N}$-phenyl- $\mathrm{N}-((2-$ vinylphenyl)sulfonyl)methacrylamide (10) $(98 \mathrm{mg}, 0.30 \mathrm{mmol}$, 1.0 equiv) and $t \mathrm{BuABX}(\mathbf{1 d})(0.207 \mathrm{~g}, 0.600 \mathrm{mmol}, 2.0$ equiv). The tube was sealed, evacuated, and backfilled with nitrogen three times. DCM (dry and freshly degassed by the freeze-pump-thaw method; $6.0 \mathrm{~mL}$ ) was then added via syringe. The mixture was stirred at room temperature for $10 \mathrm{~s}$, resulting in the immediate dissolution of the solids, and then heated at $60{ }^{\circ} \mathrm{C}$ overnight $(14 \mathrm{~h})$. The mixture was then concentrated under vacuum in the presence of silica gel. The crude product, adsorbed on silica gel, was then submitted to column chromatography $\left(\mathrm{SiO}_{2}\right.$; pentane:EtOAc 19:1 to 9:1) to afford a mixture of the desired product with tert-butyl iodobenzoic acid. To remove the latter, this mixture was diluted with DCM $(10 \mathrm{~mL})$ and washed twice with aq. $\mathrm{K}_{2} \mathrm{CO}_{3}(2.0 \mathrm{M} ; 15 \mathrm{~mL})$. The aqueous layer was extracted with DCM $(3 \times 15 \mathrm{~mL})$, and the organic extracts were dried over $\mathrm{MgSO}_{4}$, filtered, and concentrated under reduced pressure. 3-(Azidomethyl)-1-methyl- $N$-phenyl-2,3-dihydro- $1 H$-indene-1-carboxamide (11) was finally obtained as a pale yellow viscous oil (mixture of diastereoisomers, dr 9:1; $65 \mathrm{mg}, 0.19 \mathrm{mmol}, 64 \%$ ). ${ }^{1} \mathrm{H}$ NMR $\left(400 \mathrm{MHz}, \mathrm{CDCl}_{3}, \delta\right): 7.45-7.37(\mathrm{~m}, 4 \mathrm{H}), 7.38-7.31$ $(\mathrm{m}, 2 \mathrm{H}), 7.25(\mathrm{dd}, J=8.6,7.2 \mathrm{~Hz}, 2 \mathrm{H}), 7.07$ (ddt, $J=8.6,7.1$, $1.2 \mathrm{~Hz}, 1 \mathrm{H}), 6.99(\mathrm{br} \mathrm{s}, 1 \mathrm{H}), 3.70-3.59(\mathrm{~m}, 2 \mathrm{H}), 3.51(\mathrm{dq}, J=9.3$, $6.4 \mathrm{~Hz}, 1 \mathrm{H}), 3.00(\mathrm{dd}, J=13.1,7.3 \mathrm{~Hz}, 1 \mathrm{H}), 1.87(\mathrm{dd}, J=13.1,9.5 \mathrm{~Hz}$, $1 \mathrm{H}), 1.73(\mathrm{~s}, 3 \mathrm{H}) \mathrm{ppm} .{ }^{13} \mathrm{C}\left\{{ }^{1} \mathrm{H}\right\}$ NMR $\left(101 \mathrm{MHz}, \mathrm{CDCl}_{3}, \delta\right): 174.1$, 145.8, 144.6, 137.6, 129.0, 128.8, 128.6, 124.8, 124.5, 123.8, 119.8, 55.6, 54.9, 44.7, 42.6, $25.1 \mathrm{ppm}$. The signals of the ${ }^{1} \mathrm{H}$ and ${ }^{13} \mathrm{C}$ NMR spectra were in accordance with the data reported in the literature. ${ }^{8 \mathrm{c}}$

Experiment with $A B Z$ (2). Following a modified version of a reported procedure, ${ }^{8 \mathrm{C}}$ a $25 \mathrm{~mL}$ round-bottom test tube was charged with $\mathrm{N}$-phenyl- $\mathrm{N}$-((2-vinylphenyl)sulfonyl)methacrylamide (10) (98 mg, $0.30 \mathrm{mmol}, 1.0$ equiv) and $\mathrm{ABZ}(2)$ (0.265 g, $0.600 \mathrm{mmol}$, 2.0 equiv). The vial was sealed, evacuated, and backfilled with nitrogen three times. DCE (dry and freshly degassed by the freeze-pump-thaw method; $6.0 \mathrm{~mL}$ ) was then added via syringe. The mixture was heated at $100{ }^{\circ} \mathrm{C}$ under stirring overnight $(14 \mathrm{~h})$. The initially green-yellow solution became dark brown, remaining clear all the time. The mixture was allowed to cool down to room temperature, silica gel was added, and the volatiles were removed under reduced pressure with adsorption of the crude product on the silica gel itself. Column chromatography ( $\mathrm{SiO}_{2}$; pentane:EtOAC 39:1 to 19:1) afforded 3-(azidomethyl)-1methyl- $N$-phenyl-2,3-dihydro-1H-indene-1-carboxamide (11) (0.0399 g, $0.130 \mathrm{mmol}, 43 \%)$ as a pale yellow oil.

Metal-Catalyzed Azidation of Tertiary C-H bonds: Synthesis of (2-Azidopropan-2-yl)benzene (13). Experiment with $A B X$ (General Method G). Following a slightly modified procedure, ${ }^{10 \mathrm{c}}$ a flame-dried $4 \mathrm{~mL}$ round bottomed test tube was charged with $\mathrm{Fe}(\mathrm{OAc})_{2}$ (3.4 mg, $0.020 \mathrm{mmol}, 10 \mathrm{~mol} \%)$ and 2,6-bis $((S)$-4-isopropyl-4,5dihydrooxazol-2-yl)pyridine $(6.0 \mathrm{mg}, 0.020,10 \mathrm{~mol} \%)$ (this operation was performed inside a nitrogen-filled glovebox). EtOAc $(1.0 \mathrm{~mL})$ was added, and the resulting reaction mixture was stirred at room temperature for $40 \mathrm{~min}$, during which time the color changed from a colorless solution to blue. Cumene (12) $(30 \mu \mathrm{L}, 0.20 \mathrm{mmol}, 1.0$ equiv) was added, followed by ABX (1 a) $(0.17 \mathrm{~g}, 0.60 \mathrm{mmol}, 3.0$ equiv). The vial was sealed with a cap containing a PTFE septum and then stirred at $50{ }^{\circ} \mathrm{C}$ for $22 \mathrm{~h}$. The reaction was monitored by GC-MS to compare the consumption of cumene and the formation of (2-azidopropan-2yl)benzene (13), using dodecane as an internal standard (Figure S1). The area of the peak of the product was measured and normalized using the area of the dodecane peak as an internal standard (not shown on the spectra below for more clarity). Then, a ratio was done between the area of the peaks with $\mathbf{1 a}, \mathbf{1 d}$, or $\mathbf{2}$ as the reagent using $\mathbf{1 a}$ as the reference (ratio of 1.00).
Experiment with $t B u A B X(1 d)$. The reaction was performed following the general method $G$, starting from the same amounts of cumene (12), $\mathrm{Fe}(\mathrm{OAc})_{2}$, and 2,6-bis $((S)$-4-isopropyl-4,5-dihydrooxazol-2-yl)pyridine, but using $t \mathrm{BuABX}$ (1d) $(207 \mathrm{mg}, 0.600 \mathrm{mmol}$, 3.0 equiv) instead of $A B X$ (1a). The reaction was monitored by GC-MS (the chromatogram showing the reaction progress after $22 \mathrm{~h}$ is shown in Figure S2).

Experiment with $A B Z$ (2). The reaction was performed following the general method $\mathrm{G}$, starting from the same amounts of cumene (12), $\mathrm{Fe}(\mathrm{OAc})_{2}$, and 2,6-bis ((S)-4-isopropyl-4,5-dihydrooxazol-2yl)pyridine, but using ABZ (2) (265 mg, $0.600 \mathrm{mmol}, 3.0$ equiv) instead of $A B X$ (1a). The reaction was monitored by GC-MS (the chromatogram showing the reaction progress after $22 \mathrm{~h}$ is shown in Figure S3).

Cu(II)-Catalyzed Azidation of Anilines: Synthesis of 2-Azido-4-methylaniline (15). Experiment with ABX (1a) (General Method H). Following a slightly modified procedure, ${ }^{10 \mathrm{~b}}$ a $10 \mathrm{~mL}$ round bottomed test tube was charged with $\mathrm{ABX}$ (1a) (116 mg, $0.400 \mathrm{mmol}, 1.0$ equiv), $\mathrm{Cu}(\mathrm{OAc})_{2}(15 \mathrm{mg}, 0.080 \mathrm{mmol}, 10 \mathrm{~mol} \%)$, and THF $(2.7 \mathrm{~mL})$ under air. $p$-Toluidine $(14)(86 \mathrm{mg}, 0.80 \mathrm{mmol}$, 2.0 equiv) was then added, and the resulting dark mixture was stirred at room temperature under air for $12 \mathrm{~h}$. The solution was then diluted with ethyl acetate $(27 \mathrm{~mL})$, washed with saturated aqueous $\mathrm{NaHCO}_{3}$, and the two layers were separated. The organic layer was then dried over $\mathrm{MgSO}_{4}$, filtered, and concentrated in vacuo to afford a brown crude oil ( ${ }^{1} \mathrm{H}$ NMR yield with $\mathrm{CH}_{2} \mathrm{Br}_{2}(20 \mu \mathrm{L}, 0.28 \mathrm{mmol})$ as internal standard: 63\%). The latter was adsorbed on silica gel and submitted to chromatography (silica; petroleum ether:EtOAc 100:0 to 95:5) to afford pure 2-azido-4-methylaniline (15) as a brown oil $(14 \mathrm{mg}$, $0.10 \mathrm{mmol}, 24 \%$ yield; the volatility of the compound accounts for the low isolated yield). $R_{f}$ (silica, pentane:EtOAc 8:2) $0.45 .{ }^{1} \mathrm{H}$ NMR $\left(400 \mathrm{MHz}, \mathrm{CDCl}_{3}, \delta\right): 6.84(\mathrm{~d}, J=1.9 \mathrm{~Hz}, 1 \mathrm{H}), 6.76(\mathrm{dd}, J=8.1,1.9$ $\mathrm{Hz}, 1 \mathrm{H}), 6.61(\mathrm{~d}, J=8.0 \mathrm{~Hz}, 1 \mathrm{H}), 3.67(\mathrm{~s}, 2 \mathrm{H}), 2.27(\mathrm{~s}, 3 \mathrm{H})$. The signals of the ${ }^{1} \mathrm{H}$ NMR spectra were in accordance with the data reported in the literature. ${ }^{10 \mathrm{~b}}$

Experiment with $t B \cup A B X$ (1d). The reaction was performed following the general method $\mathrm{H}$, starting from the same amounts of $p$-toluidine (14) and $\mathrm{Cu}(\mathrm{OAc})_{2}$, but using $t \mathrm{BuABX}(1 \mathrm{~d})(138 \mathrm{mg}$, $0.400 \mathrm{mmol}, 1.0$ equiv) instead of $\mathrm{ABX}$ (1a). 2-Azido-4-methylaniline (15) was generated in $62 \%$ yield (based on ${ }^{1} \mathrm{H}$ NMR and using $\mathrm{CH}_{2} \mathrm{Br}_{2}(20 \mu \mathrm{L}, 0.28 \mathrm{mmol})$ as an internal standard $)$. Upon column chromatography, it was isolated as a brown oil $(15 \mathrm{mg}, 0.10 \mathrm{mmol}$, $25 \%$ yield; the volatility of the compound accounts for the low isolated yield).

Experiment with $A B Z$ (2). The reaction was performed following the general method $\mathrm{H}$, starting from the same amounts of $p$-toluidine (14) and $\mathrm{Cu}(\mathrm{OAc})_{2}$, but using $\mathrm{ABZ}$ (2) $(177 \mathrm{mg}, 0.400 \mathrm{mmol}$, 1.0 equiv) instead of $A B X$ (1a). 2-Azido-4-methylaniline (15) was generated in $72 \%$ yield (based on ${ }^{1} \mathrm{H}$ NMR and using $\mathrm{CH}_{2} \mathrm{Br}_{2}(20 \mu \mathrm{L}$, $0.28 \mathrm{mmol}$ ) as an internal standard). Upon column chromatography, it was isolated as a brown oil ( $16 \mathrm{mg}, 0.10 \mathrm{mmol}, 26 \%$ yield; the volatility of the compound accounts for the low isolated yield).

$\mathrm{Cu}(\mathrm{I})$-Catalyzed Intramolecular Alkene Aminoazidation: Synthesis of 3-(Azidomethyl)-2-methoxy-3,4-dihydroisoquinolin-1(2H)-one (17). Experiment with tBuABX (1d) (General Method I). Following a reported procedure, ${ }^{10 \mathrm{~h}}$ a $25 \mathrm{~mL}$ round-bottom test tube was charged with 2 -allyl- $N$-methoxybenzamide $(16)(57 \mathrm{mg}$, $0.30 \mathrm{mmol}, 1.0$ equiv), $t \mathrm{BuABX}$ (1d) $(0.124 \mathrm{~g}, 0.360 \mathrm{mmol}, 1.2$ equiv), and $\mathrm{CuOAc}(3.7 \mathrm{mg}, 0.030 \mathrm{mmol}, 10 \mathrm{~mol} \%)$. The vessel was sealed, evacuated, and backfilled with nitrogen ( 3 times). Acetonitrile (dry and degassed by purging it with $\mathrm{Ar}$ for $15 \mathrm{~min} ; 3.0 \mathrm{~mL}$ ) was added via syringe, giving initially a pale blue suspension. The latter was heated at $60{ }^{\circ} \mathrm{C}$ under stirring for $2 \mathrm{~h}$. After $5 \mathrm{~min}$ at this temperature, the suspension had turned into a clear blue-green solution; a precipitate was formed after $1.5 \mathrm{~h}$. After $2 \mathrm{~h}$, TLC analysis (pentane:EtOAc 3:1) showed the complete consumption of the starting material. The reaction mixture was poured into sat. aq. $\mathrm{NaHCO}_{3}(15 \mathrm{~mL})$, and the aqueous layer was extracted with EtOAc $(3 \times 15 \mathrm{~mL})$. The combined organic extracts were washed with brine, dried over $\mathrm{MgSO}_{4}$, filtered, and concentrated under vacuum to afford a pale yellow crude solid. 
The latter was submitted to column chromatography $\left(\mathrm{SiO}_{2}\right.$; pentane:EtOAc 94:4 to 50:50) to afford 3-(azidomethyl)-2methoxy-3,4-dihydroisoquinolin-1(2H)-one (17) (95\% pure; $0.054 \mathrm{~g}$, $0.23 \mathrm{mmol}, 73 \%$ yield) as a colorless oil. ${ }^{1} \mathrm{H}$ NMR $\left(400 \mathrm{MHz}, \mathrm{CDCl}_{3}\right.$, $\delta): 8.12(\mathrm{dd}, J=7.8,1.4 \mathrm{~Hz}, 1 \mathrm{H}), 7.48(\mathrm{td}, J=7.5,1.5 \mathrm{~Hz}, 1 \mathrm{H}), 7.37$ (tt, $J=7.7,1.1 \mathrm{~Hz}, 1 \mathrm{H}), 7.22(\mathrm{dd}, J=7.4,1.3 \mathrm{~Hz}, 1 \mathrm{H}), 4.07(\mathrm{ddt}, J=$ 8.5, 5.9, $3.8 \mathrm{~Hz}, 1 \mathrm{H}$ ), 3.93 (s, $3 \mathrm{H}$ ), 3.67 (ddd, $J=12.2,3.6,0.8 \mathrm{~Hz}$, $1 \mathrm{H}), 3.48-3.31(\mathrm{~m}, 2 \mathrm{H}), 3.18(\mathrm{dd}, J=16.5,4.0 \mathrm{~Hz}, 1 \mathrm{H}) \mathrm{ppm}$. ${ }^{13} \mathrm{C}\left\{{ }^{1} \mathrm{H}\right\}$ NMR $\left(101 \mathrm{MHz}, \mathrm{CDCl}_{3}\right.$; one aromatic signal was not resolved, $\delta$ ): 163.2, 134.8, 132.8, 128.2, 128.0, 127.5, 63.1, 57.9, 50.5, $31.4 \mathrm{ppm}$. The signals of the ${ }^{1} \mathrm{H}$ and ${ }^{13} \mathrm{C}$ NMR spectra were in accordance with the data reported in the literature. ${ }^{10 \mathrm{~h}}$ In the ${ }^{1} \mathrm{H}$ NMR spectrum, all peaks were shifted $+0.17 \mathrm{ppm}$ with respect to the ones in the literature.

Experiment with $A B Z$ (2). The reaction was performed following the general method I, starting from the same amounts of 2-allyl- $N$ methoxybenzamide (16) and CuOAc, but using $\mathrm{ABZ}$ (2) (159 mg, $0.360 \mathrm{mmol}, 2.0$ equiv) instead of $t \mathrm{BuABX}(1 \mathrm{~d})$. The crude product was purified by column chromatography $\left(\mathrm{SiO}_{2}\right.$; pentane:EtOAc 94:4 to 50:50), followed by preparative TLC $\left(\mathrm{SiO}_{2}, 20 \times 20\right.$ sq. cm; hexane:EtOAc 50:50), to afford 3-(azidomethyl)-2-methoxy-3,4dihydroisoquinolin-1 $(2 \mathrm{H})$-one (17) (0.060 g, $0.26 \mathrm{mmol}, 86 \%$ yield) as a colorless oil.

\section{METHODS FOR SAFETY STUDIES}

The stability of the compounds was determined by DSC. A few milligrams of samples were sealed in gold plated pressure resistant crucibles. The crucibles were heated in an oven from 30 to $400{ }^{\circ} \mathrm{C}$ at a rate of $4{ }^{\circ} \mathrm{C} / \mathrm{min}$. The $T_{\text {onset }}$ (temperature at which an exothermic signal is detected), the decomposition energy, and the peak temperature were recorded and compared. The provided data are the mean of the two measurements.

The sensitivity to friction and impact as well as the energy of decomposition measured by DSC were determined for the Zhdankin reactive (1a) as well as for $t \mathrm{Bu}-\mathrm{ABX}(\mathbf{1 d})$ and $\mathrm{ABZ}$ (2).

The sensitivity to impact or shock (Fallhammer test) consists of subjecting $40 \mu \mathrm{L}$ of substance to different drop-weight impacts. The impact energy is varied by the selection of drop height and weight. A drop weight of $1 \mathrm{~kg}$ was used for this study. The test is positive if at least one positive result (detonation, fume, fire, or sparks) in a series of six measurements is obtained. Here a microphone was used for the detection. The limiting impact energy is the lowest energy at which there is still a positive result. The tests were performed with $1 \mathrm{~J}$ steps.

The sensitivity to friction was measured with a BAM friction apparatus. A porcelain plate with $10 \mathrm{ul}$ of test substance was moved by an electric motor against a stationary porcelain peg. The load corresponded to a friction force of $360 \mathrm{~N}$. The test was then repeated on Alox (aluminum oxide) plates, as many false positives were measured with porcelain plates.

\section{ASSOCIATED CONTENT}

\section{S Supporting Information}

The Supporting Information is available free of charge on the ACS Publications website at DOI: 10.1021/acs.joc.8b02068.

Reactions schemes for the synthesis of starting materials and reagents, reaction optimization details, accident report, and chromatogram and NMR spectra of new compounds or reported compounds synthesized using modified methods (PDF)

\section{AUTHOR INFORMATION}

\section{Corresponding Author}

* jerome.waser@epfl.ch

\section{ORCID $\odot$}

Thierry Meyer: 0000-0003-2546-9107 Jerome Waser: 0000-0002-4570-914X

\section{Author Contributions}

${ }^{\perp}$ S.A. and J.P. contributed equally to this work.

\section{Notes}

The authors declare no competing financial interest.

\section{ACKNOWLEDGMENTS}

We thank ERC (European Research Council, Starting Grant iTools4MC, number 334840), SNF (sinergia CRSII5_171026), and EPFL for financial support. Mr. Christian Aeby, Ms. Delphine Berset, and Mr. Daniel Steiner are acknowledged for performing the safety assays at TÜV SÜD Schweiz AG.

\section{REFERENCES}

(1) Bräse, S.; Banert, K. Organic Azides: Syntheses and Applications; John Wiley \& Sons: Chichester, 2010.

(2) Bräse, S.; Gil, C.; Knepper, K.; Zimmermann, V. Organic Azides: An Exploding Diversity of a Unique Class of Compounds. Angew. Chem., Int. Ed. 2005, 44, 5188.

(3) (a) Wirth, T. Hypervalent Iodine Chemistry in Synthesis: Scope and New Directions. Angew. Chem., Int. Ed. 2005, 44, 3656. (b) Zhdankin, V. V.; Stang, P. J. Chemistry of Polyvalent Iodine. Chem. Rev. 2008, 108, 5299. (c) Yoshimura, A.; Zhdankin, V. V. Advances in Synthetic Applications of Hypervalent Iodine Compounds. Chem. Rev. 2016, 116, 3328. (d) Li, Y.; Hari, D. P.; Vita, M. V.; Waser, J. Cyclic Hypervalent Iodine Reagents for Atom-Transfer Reactions: Beyond Trifluoromethylation. Angew. Chem., Int. Ed. 2016, 55, 4436.

(4) Selected examples: (a) Moriarty, R. M.; Vaid, R. K.; Ravikumar, V. T.; Vaid, B. K.; Hopkins, T. E. Hypervalent iodine oxidation: $\alpha$ Functionalization of $\beta$-dicarbonyl compounds using iodosobenzene. Tetrahedron 1988, 44, 1603. (b) Magnus, P.; Barth, L. Oxidative addition of azide anion to triisopropylsilyl enol ethers: synthesis of $\alpha$ azido ketones. Tetrahedron Lett. 1992, 33, 2777. (c) Magnus, P.; Lacour, J. New trialkylsilyl enol ether chemistry. Direct. $\beta$-azido functionalization of triisopropylsilyl enol ethers. J. Am. Chem. Soc. 1992, 114, 767

(5) (a) Zhdankin, V. V.; Kuehl, C. J.; Krasutsky, A. P.; Formaneck, M. S.; Bolz, J. T. Preparation and chemistry of stable azidoiodinanes: 1-Azido-3,3-bis(trifluoromethyl)-3-(1H)-1,2-benziodoxol and 1Azido-1,2-benziodoxol-3-(1H)-one. Tetrahedron Lett. 1994, 35, 9677. (b) Krasutsky, A. P.; Kuehl, C. J.; Zhdankin, V. V. Direct Azidation of Adamantane and Norbornane by Stable Azidoiodinanes. Synlett 1995, 1995, 1081. (c) Zhdankin, V. V.; Krasutsky, A. P.; Kuehl, C. J.; Simonsen, A. J.; Woodward, J. K.; Mismash, B.; Bolz, J. T. Preparation, X-ray Crystal Structure, and Chemistry of Stable Azidoiodinanes Derivatives of Benziodoxole. J. Am. Chem. Soc. 1996, $118,5192$.

(6) Akai, S.; Okuno, T.; Egi, M.; Takada, T.; Tohma, H.; Kita, Y. Preparation of Novel Cyclic Hypervalent Iodine(III) Compounds Having Azido, Cyano, and Nitrato Ligands. Heterocycles 1996, $42,47$.

(7) (a) Deng, Q.-H.; Bleith, T.; Wadepohl, H.; Gade, L. H. Enantioselective Iron-Catalyzed Azidation of $\beta$-Keto Esters and Oxindoles. J. Am. Chem. Soc. 2013, 135, 5356. (b) Vita, M. V.; Waser, J. Azidation of $\beta$-Keto Esters and Silyl Enol Ethers with a Benziodoxole Reagent. Org. Lett. 2013, 15, 3246. (c) Zhang, B.; Studer, A. Stereoselective Radical Azidooxygenation of Alkenes. Org. Lett. 2013, 15, 4548.

(8) Selected examples: (a) Kong, W.; Merino, E.; Nevado, C. Arylphosphonylation and Arylazidation of Activated Alkenes. Angew. Chem., Int. Ed. 2014, 53, 5078. (b) Fuentes, N.; Kong, W.; FernándezSánchez, L.; Merino, E.; Nevado, C. Cyclization Cascades via $N$ Amidyl Radicals toward Highly Functionalized Heterocyclic Scaffolds. J. Am. Chem. Soc. 2015, 137, 964. (c) Kong, W.; Fuentes, N.; GarcíaDomínguez, A.; Merino, E.; Nevado, C. Stereoselective Synthesis of Highly Functionalized Indanes and Dibenzocycloheptadienes through Complex Radical Cascade Reactions. Angew. Chem., Int. Ed. 2015, 54, 2487. (d) Shinomoto, Y.; Yoshimura, A.; Shimizu, H.; Yamazaki, M.; 
Zhdankin, V. V.; Saito, A. Tetra- $n$-butylammonium Iodide Catalyzed $\mathrm{C}-\mathrm{H}$ Azidation of Aldehydes with Thermally Stable Azidobenziodoxolone. Org. Lett. 2015, 17, 5212.

(9) Selected examples: (a) Fumagalli, G.; Rabet, P. T. G.; Boyd, S.; Greaney, M. F. Three-Component Azidation of Styrene-Type Double Bonds: Light-Switchable Behavior of a Copper Photoredox Catalyst. Angew. Chem., Int. Ed. 2015, 54, 11481. (b) Rabet, P. T.; Fumagalli, G.; Boyd, S.; Greaney, M. F. Benzylic C-H Azidation Using the Zhdankin Reagent and a Copper Photoredox Catalyst. Org. Lett. 2016, 18, 1646. (c) Wang, Y.; Li, G.-X.; Yang, G.; He, G.; Chen, G. A visible-light-promoted radical reaction system for azidation and halogenation of tertiary aliphatic $\mathrm{C}-\mathrm{H}$ bonds. Chem. Sci. 2016, 7, 2679.

(10) Selected examples: (a) Yin, H.; Wang, T.; Jiao, N. CopperCatalyzed Oxoazidation and Alkoxyazidation of Indoles. Org. Lett. 2014, 16, 2302. (b) Fan, Y.; Wan, W.; Ma, G.; Gao, W.; Jiang, H.; $\mathrm{Zhu}, \mathrm{S}$.; Hao, J. Room-temperature $\mathrm{Cu}(\mathrm{II})$-catalyzed aromatic $\mathrm{C}-\mathrm{H}$ azidation for the synthesis of ortho-azido anilines with excellent regioselectivity. Chem. Commun. 2014, 50, 5733. (c) Sharma, A.; Hartwig, J. F. Metal-catalysed azidation of tertiary $\mathrm{C}-\mathrm{H}$ bonds suitable for late-stage functionalization. Nature 2015, 517, 600. (d) Karimov, R. R.; Sharma, A.; Hartwig, J. F. Late Stage Azidation of Complex Molecules. ACS Cent. Sci. 2016, 2, 715. (e) Yuan, Y.-A.; Lu, D.-F.; Chen, Y.-R.; Xu, H. Iron-Catalyzed Direct Diazidation for a Broad Range of Olefins. Angew. Chem., Int. Ed. 2016, 55, 534. (f) Lonca, G. H.; Ong, D. Y.; Tran, T. M. H.; Tejo, C.; Chiba, S.; Gagosz, F. Anti-Markovnikov Hydrofunctionalization of Alkenes: Use of a Benzyl Group as a Traceless Redox-Active Hydrogen Donor. Angew. Chem., Int. Ed. 2017, 56, 11440. (g) Hendrick, C. E.; Bitting, K. J.; Cho, S.; Wang, Q. Site-Selective Copper-Catalyzed Amination and Azidation of Arenes and Heteroarenes via Deprotonative Zincation. J. Am. Chem. Soc. 2017, 139, 11622. (h) Shen, K.; Wang, Q. Copper-Catalyzed Alkene Aminoazidation as a Rapid Entry to 1,2Diamines and Installation of an Azide Reporter onto Azahetereocycles. J. Am. Chem. Soc. 2017, 139, 13110.

(11) Alazet, S.; Le Vaillant, F.; Nicolai, S.; Courant, T.; Waser, J. Divergent Access to $(1,1)$ and $(1,2)$-Azidolactones from Alkenes using Hypervalent Iodine Reagents. Chem. - Eur. J. 2017, 23, 9501.

(12) Selected examples: (a) Kim, S.; Ho Park, J. An episulfonium ion mediated ring expansion of 1-alkenylcycloakanols. Tetrahedron Lett. 1989, 30, 6181. (b) Nemoto, H.; Shiraki, M.; Fukumoto, K. Novel Access to Neopentyl-Type Halogenated Cyclopentanoids via Olefinic Cyclobutanols. J. Org. Chem. 1996, 61, 1347. (c) Zhang, E.; Fan, C. A.; Tu, Y. Q.; Zhang, F. M.; Song, Y. L. Organocatalytic Asymmetric Vinylogous $\alpha$-Ketol Rearrangement: Enantioselective Construction of Chiral All-Carbon Quaternary Stereocenters in Spirocyclic Diketones via Semipinacol-Type 1,2-Carbon Migration. J. Am. Chem. Soc. 2009, 131, 14626. (d) Romanov-Michailidis, F.; Guenee, L.; Alexakis, A. Enantioselective Organocatalytic Fluorination-Induced Wagner-Meerwein Rearrangement. Angew. Chem., Int. Ed. 2013, 52, 9266. (e) Yin, Q.; You, S. L. Asymmetric Chlorination/Ring Expansion for the Synthesis of $\alpha$-Quaternary Cycloalkanones. Org. Lett. 2014, 16, 1810. (f) Xu, M.-H.; Dai, K.-L.; Tu, Y.-Q.; Zhang, X.-M.; Zhang, F.-M.; Wang, S.-H. A catalytic allylic cation-induced intermolecular allylation-semipinacol rearrangement. Chem. Commun. 2018, 54, 7685.

(13) Selected examples: (a) Clark, G. R.; Thiensathit, S. A palladium-catalyzed rearrangement of 1-vinyl-1-cyclobutanols. Tetrahedron Lett. 1985, 26, 2503. (b) Trost, B. M.; Yasukata, T. A Catalytic Asymmetric Wagner-Meerwein Shift. J. Am. Chem. Soc. 2001, 123, 7162. (c) Shu, X. Z.; Zhang, M.; He, Y.; Frei, H.; Toste, F. D. Dual Visible Light Photoredox and Gold-Catalyzed Arylative Ring Expansion. J. Am. Chem. Soc. 2014, 136, 5844. (d) Lukamto, D. H.; Gaunt, M. J. Enantioselective Copper-Catalyzed Arylation-Driven Semipinacol Rearrangement of Tertiary Allylic Alcohols with Diaryliodonium Salts. J. Am. Chem. Soc. 2017, 139, 9160. (e) Wu, H.; Wang, Q.; Zhu, J. Copper-Catalyzed Enantioselective Domino Arylation/Semipinacol Rearrangement of Allylic Alcohols with Diaryliodonium Salts. Chem. - Eur. J. 2017, 23, 13037.
(14) Selected examples: (a) Sahoo, B.; Li, J. L.; Glorius, F. VisibleLight Photoredox-Catalyzed Semipinacol-Type Rearrangement: Trifluoromethylation/Ring Expansion by a Radical-Polar Mechanism. Angew. Chem., Int. Ed. 2015, 54, 11577. (b) Kwon, S. J.; Kim, D. Y. Visible Light Photoredox-Catalyzed Arylative Ring Expansion of 1-(1Arylvinyl)cyclobutanol Derivatives. Org. Lett. 2016, 18, 4562. (c) Zhang, J.-J.; Cheng, Y.-B.; Duan, X.-H. Metal-Free Oxidative Decarboxylative Acylation/Ring Expansion of Vinylcyclobutanols with $\alpha$-Keto Acids by Visible Light Photoredox Catalysis. Chin. J. Chem. 2017, 35, 311.

(15) For examples of amination/azidation not involving ringexpansion, see: (a) Zhang, E.; Tu, Y.-Q.; Fan, C.-A.; Zhao, X.; Jiang, Y.-J.; Zhang, S.-Y. Tandem Aziridination/Rearrangement Reaction of Allylic Alcohols: An Efficient Approach to 2-Quaternary Mannich Bases. Org. Lett. 2008, 10, 4943. (b) Chen, Z.-M.; Zhang, Z.; Tu, Y.Q.; Xu, M.-H.; Zhang, F.-M.; Li, C.-C.; Wang, S.-H. A Mn(III)/ TEMPO-co-mediated tandem azidation-1,2-carbon migration reaction of allylic silyl ethers. Chem. Commun. 2014, 50, 10805. (c) Ren, R.; Zhao, H.; Huan, L.; Zhu, C. Manganese-Catalyzed Oxidative Azidation of Cyclobutanols: Regiospecific Synthesis of Alkyl Azides by C-C Bond. Cleavage. Angew. Chem., Int. Ed. 2015, 54, 12692.

(16) These results have been posted on the preprint server ChemRXiv: Alazet, S.; Preindl, J.; Simonet-Davin, R.; Nicolai, S.; Nanchen, A.; Meyer, T.; Waser, J. Cyclic Hypervalent Iodine Reagents for Azidation: Safer Reagents and Photoredox Catalyzed Ring Expansion. ChemRXiv 2018, DOI: 10.26434/chemrxiv.6946058.v1.

(17) (a) Ahn, B. T.; McMillin, D. R. Studies of photoinduced electron transfer from bis(2,9-dimethyl-1,10-phenanthroline)copper(I). Inorg. Chem. 1978, 17, 2253. (b) Dietrichbuchecker, C. O.; Marnot, P. A.; Sauvage, J. P.; Kirchhoff, J. R.; McMillin, D. R. Bis(2,9diphenyl-1,10-phenanthroline)copper(I): a copper complex with a long-lived charge-transfer excited state. J. Chem. Soc., Chem. Commun. 1983, 0, 513.

(18) See the SI for a detailed accident report.

(19) In addition, all reagents were stable under ambient light in absence of a catalyst.

(20) Stoessel, F. Thermal Safety of Chemical Processes; Wiley-VCH, 2008.

(21) Mage, L.; Baati, N.; Nanchen, A.; Stoessel, F.; Meyer, Th. Process Saf. Environ. Prot. 2017, 110, 43.

(22) $\mathrm{TD}_{24}$ was estimated under the following specific assumptions (zero order kinetic $=$ worst-case scenario; heat release rate at onset 20 $\mathrm{W} / \mathrm{kg}$; activation energy $=100 \mathrm{~kJ} \mathrm{~mol}^{-1}$; heat capacity $=2 \mathrm{~J} \mathrm{~g}^{-1} \mathrm{~K}^{-1}$ ).

(23) The energy release is assumed to be comparable to the potential energy $(E=\mathrm{mgH})$ of the system for (1) a falling of the reaction flask on the ground ( $1 \mathrm{~m}$ height, total mass of $350 \mathrm{~g}$ ) and (2) the falling of a spatula inside the reaction flask during manipulation $(10 \mathrm{~cm}$ height, mass of $25 \mathrm{~g}$ ).

(24) See the SI for details.

(25) As isolation of volatile azide $\mathbf{1 3}$ is difficult on a small scale, relative yields of 13 obtained using $A B X(1 \mathrm{a}), t \mathrm{Bu}-\mathrm{ABX}(\mathbf{1 d})$, and ABZ (2) were compared by GC-MS.

(26) Yields determined by NMR.

(27) Iskra, J.; Murphree, S. S. Rapid aerobic iodination of arenes mediated by hypervalent iodine in fluorinated solvents. Tetrahedron Lett. 2017, 58, 645.

(28) Bedrac, L.; Iskra, J. Iodine(I) Reagents in Hydrochloric AcidCatalyzed Oxidative Iodination of Aromatic Compounds by Hydrogen Peroxide and Iodine. Adv. Synth. Catal. 2013, 355, 1243.

(29) Sun, M.-X.; Wang, Y.-F.; Xu, B.-H.; Ma, X.-Q.; Zhang, S.-J. A metal-free direct $\mathrm{C}\left(\mathrm{sp}^{3}\right)-\mathrm{H}$ cyanation reaction with cyanobenziodoxolones. Org. Biomol. Chem. 2018, 16, 1971.

(30) Liu, Y.; Yeung, Y.-Y. Synthesis of Macrocyclic Ketones through Catalyst-Free Electrophilic Halogen-Mediated Semipinacol Rearrangement: Application to the Total Synthesis of ( \pm )-Muscone. Org. Lett. 2017, 19, 1422. 
(31) Zhang, Y.; Li, J.; Li, X.; He, J. Regio-Specific Polyacetylenes Synthesized from Anionic Polymerizations of Template Monomers. Macromolecules 2014, 47, 6260.

(32) Wu, J.; Yoshikai, N. Cobalt-Catalyzed Alkenylzincation of Unfunctionalized Alkynes. Angew. Chem., Int. Ed. 2016, 55, 336.

(33) Ojha, D. P.; Prabhu, K. R. Regioselective Synthesis of Vinyl Halides, Vinyl Sulfones, and Alkynes: A Tandem Intermolecular Nucleophilic and Electrophilic Vinylation of Tosylhydrazones. Org. Lett. 2015, 17, 18.

(34) Gonzalez-de-Castro, A.; Xiao, J. Green and Efficient: IronCatalyzed Selective Oxidation of Olefins to Carbonyls with $\mathrm{O}_{2}$. J. Am. Chem. Soc. 2015, 137, 8206.

(35) Gao, F.; Hoveyda, A. H. $\alpha$-Selective Ni-Catalyzed Hydroalumination of Aryl- and Alkyl-Substituted Terminal Alkynes: Practical Syntheses of Internal Vinyl Aluminums, Halides, or Boronates. J. Am. Chem. Soc. 2010, 132, 10961.

(36) Liu, Y.; Tse, Y.-L. S.; Kwong, F. Y.; Yeung, Y.-Y. Accessing Axially Chiral Biaryls via Organocatalytic Enantioselective DynamicKinetic Resolution-Semipinacol Rearrangement. ACS Catal. 2017, 7, 4435.

(37) Rosiak, A.; Frey, W.; Christoffers, J. Synthesis of Tetrahydropyran-4-ones and Thiopyran-4-ones from Donor-Substituted $\alpha$ Bromostyrene Derivatives. Eur. J. Org. Chem. 2006, 2006, 4044.

(38) Overman, L. E.; Mendelson, L. T.; Jacobsen, E. J. Synthesis applications of aza-Cope rearrangements. 12. Applications of cationic aza-Cope rearrangements for alkaloid synthesis. Stereoselective preparation of cis-3a-aryloctahydroindoles and a new short route to Amaryllidaceae alkaloids. J. Am. Chem. Soc. 1983, 105, 6629.

(39) Nelb, R. G., II; Stille, J. K. Three different consecutive orbital symmetry-controlled reactions in the novel stereospecific synthesis of 1-phenyl-4-(1-phenylethenyl)naphthalene. The reaction of 3,4diphenylthiophene 1,1-dioxide with ethynylbenzene. J. Am. Chem. Soc. 1976, 98, 2834.

(40) Spaggiari, A.; Vaccari, D.; Davoli, P.; Torre, G.; Prati, F. A Mild Synthesis of Vinyl Halides and gem-Dihalides Using Triphenyl Phosphite-Halogen-Based Reagents. J. Org. Chem. 2007, 72, 2216.

(41) Romanov-Michailidis, F.; Romanova-Michaelides, M.; Pupier, M.; Alexakis, A. Enantioselective Halogenative Semi-Pinacol Rearrangement: Extension of Substrate Scope and Mechanistic Investigations. Chem. - Eur. J. 2015, 21, 5561.

(42) Ohta, Y.; Chiba, H.; Oishi, S.; Fujii, N.; Ohno, H. Construction of Nitrogen Heterocycles Bearing an Aminomethyl Group by CopperCatalyzed Domino Three-Component Coupling-Cyclization. J. Org. Chem. 2009, 74, 7052.

(43) Debnath, S.; Mondal, S. One-Pot Sonogashira CouplingCyclization toward Regioselective Synthesis of Benzosultams. J. Org. Chem. 2015, 80, 3940.

(44) Szadkowska, A.; Żukowska, K.; Pazio, A. E.; Woźniak, K.; Kadyrov, R.; Grela, K. Ruthenium Olefin Metathesis Catalysts Containing Six-Membered Sulfone and Sulfonamide Chelating Rings. Organometallics 2011, 30, 1130.

(45) Su, X.; Huang, H.; Hong, W.; Cui, J.; Yu, M.; Li, Y. Alkyl radical triggered in situ $\mathrm{SO}_{2}$-capture cascades. Chem. Commun. 2017, 53, 13324.

(46) Brady, R. M.; Khakham, Y.; Lessene, G.; Baell, J. B. Benzoylureas as removable cis amide inducers: synthesis of cyclic amides via ring closing metathesis (RCM). Org. Biomol. Chem. 2011, 9, 656 .

(47) Dydio, P.; Detz, R. J.; de Bruin, B.; Reek, J. N. H. Beyond Classical Reactivity Patterns: Hydroformylation of Vinyl and Allyl Arenes to Valuable $\beta$ - and $\gamma$-Aldehyde Intermediates Using Supramolecular Catalysis. J. Am. Chem. Soc. 2014, 136, 8418.

(48) Dai, H.; Yu, C.; Wang, Z.; Yan, H.; Lu, C. Solvent-Controlled, Tunable $\beta$-OAc and $\beta-\mathrm{H}$ Elimination in $\mathrm{Rh}(\mathrm{III})$-Catalyzed Allyl Acetate and Aryl Amide Coupling via $\mathrm{C}-\mathrm{H}$ Activation. Org. Lett. 2016, 18, 3410. 


\section{Content}

1. Synthesis of the azidation hypervalent iodine reagents ........................................................ 2

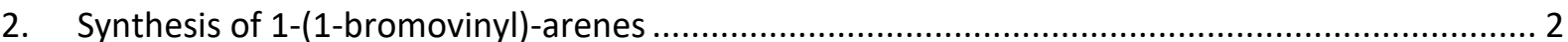

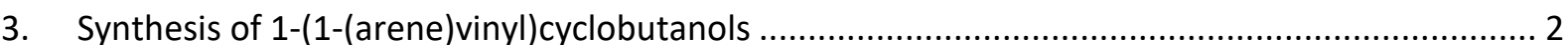

4. Synthesis of (1-(1-(arene)vinyl)cyclobutoxy)trimethylsilanes ................................................. 3

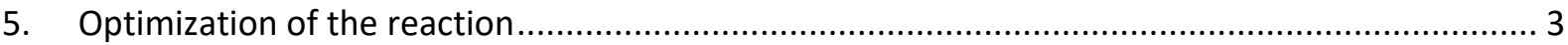

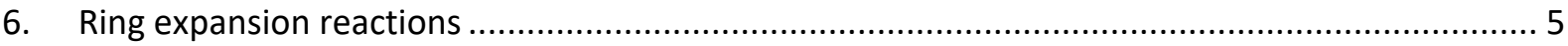

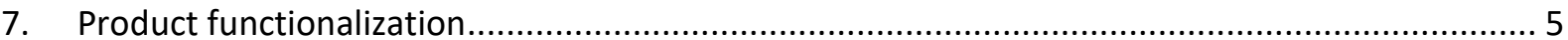

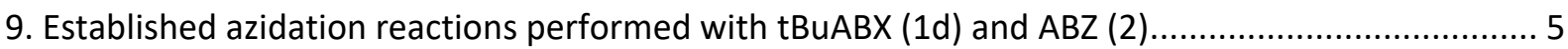

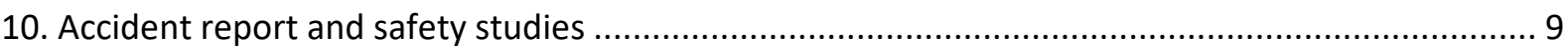

11 Spectra of compounds synthesized by modified methods and of new compounds ............... 11 
1. Synthesis of the azidation hypervalent iodine reagents

Synthesis of ABX (1a)

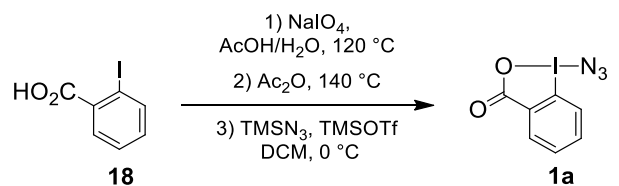

Synthesis of $t \mathrm{BuABX}(1 \mathrm{~d})$

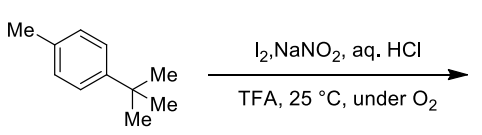

19

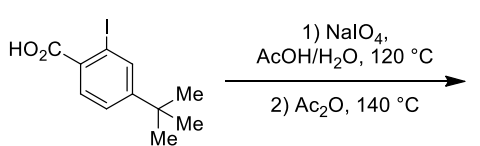

21

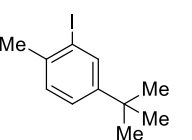

20

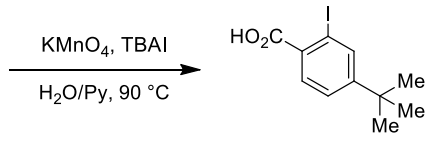

21

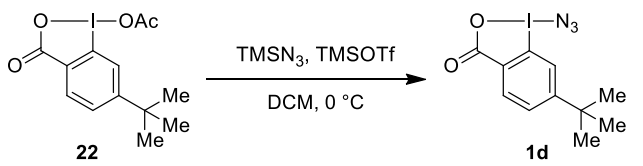

Synthesis of ABZ (2).

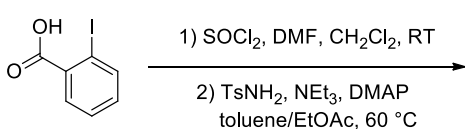

18

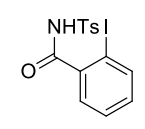

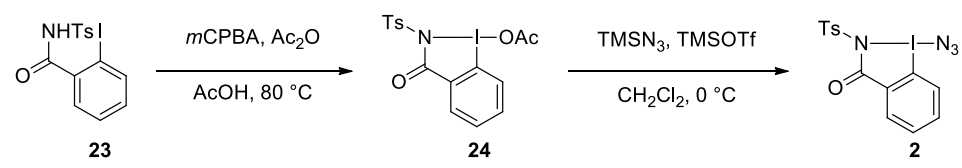

2. Synthesis of 1-(1-bromovinyl)-arenes

\section{General method A:}

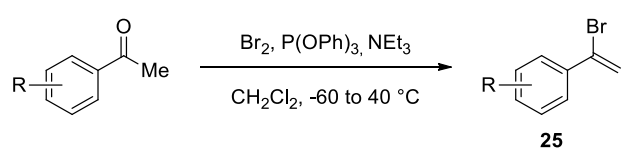

General method B:

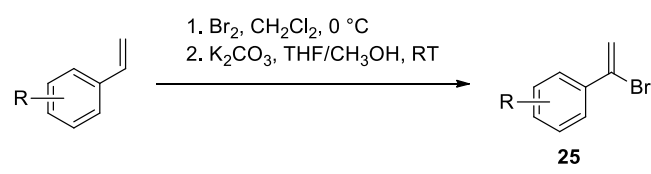

3. Synthesis of 1-(1-(arene)vinyl)cyclobutanols

\section{General method C:}




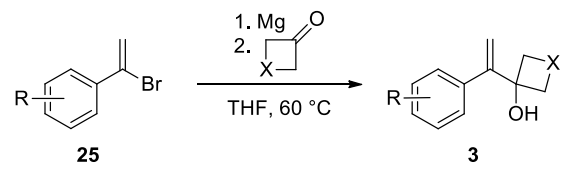

1-(3,4-dihydronaphthalen-1-yl)cyclobutanols (3s)

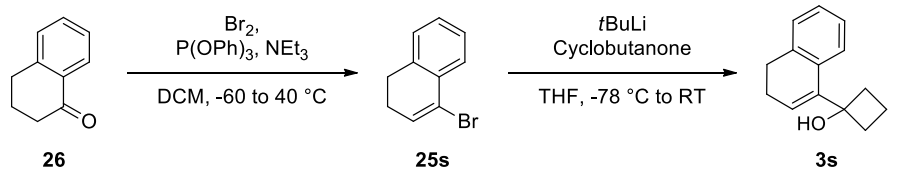

4. Synthesis of (1-(1-(arene)vinyl)cyclobutoxy)trimethylsilanes

General procedure D:

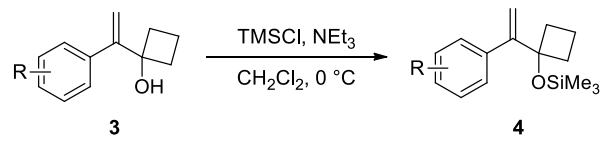

5. Optimization of the reaction

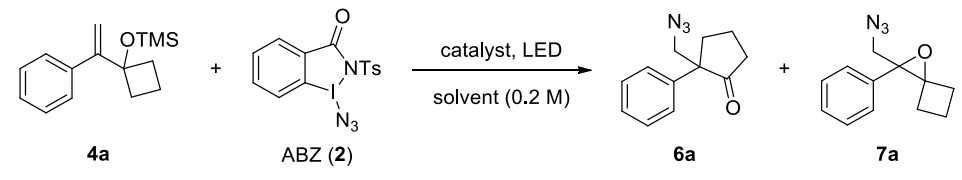

\begin{tabular}{|c|c|c|c|c|c|}
\hline Entry $^{a}$ & $\mathrm{ABZ}(\mathrm{XX})$ & Catalyst & LED & Solvent & Result $^{b}$ \\
\hline 1 & 2 equiv. & $\mathrm{Cu}(\mathrm{dap})_{2} \mathrm{Cl}(0.5 \mathrm{~mol} \%)$ & green & DMF & $56 \%(6 a)$ \\
\hline 2 & 2 equiv. & $\mathrm{Cu}(\text { dap })_{2} \mathrm{Cl}(0.5 \mathrm{~mol} \%)$ & green & $\mathrm{CH}_{3} \mathrm{OH}$ & $70 \%(6 a)$ \\
\hline 3 & 2 equiv. & $\mathrm{Cu}(\text { dap })_{2} \mathrm{Cl}(0.5 \mathrm{~mol} \%)$ & green & DMA & $65 \%(6 a)$ \\
\hline 4 & 2 equiv. & $\mathrm{Cu}(\text { dap })_{2} \mathrm{Cl}(0.5 \mathrm{~mol} \%)$ & green & $\mathrm{CH}_{2} \mathrm{Cl}_{2}$ & $56 \%(6 a)$ \\
\hline 5 & 2 equiv. & $\mathrm{Cu}(\text { dap })_{2} \mathrm{Cl}(0.5 \mathrm{~mol} \%)$ & green & DMSO & $74 \%(6 a: 7 a=1: 3)$ \\
\hline 6 & 2 equiv. & $\mathrm{Cu}(\text { dap })_{2} \mathrm{Cl}(0.5 \mathrm{~mol} \%)$ & green & $\mathrm{CH}_{3} \mathrm{CN}$ & $85 \%(6 a)$ \\
\hline 7 & $\begin{array}{l}1.2 \\
\text { equiv. }\end{array}$ & $\mathrm{Cu}(\text { dap })_{2} \mathrm{Cl}(0.5 \mathrm{~mol} \%)$ & green & $\mathrm{CH}_{3} \mathrm{CN}$ & $88 \%(90 \%)^{c}(6 a)$ \\
\hline 8 & $\begin{array}{l}1.2 \\
\text { equiv. }\end{array}$ & $\mathrm{Ru}(\mathrm{bpy})_{3} \mathrm{Cl}_{2}(1 \mathrm{~mol} \%)$ & green & $\mathrm{CH}_{3} \mathrm{CN}$ & $74 \%(6 a)$ \\
\hline 9 & $\begin{array}{l}1.2 \\
\text { equiv. }\end{array}$ & $\mathrm{Ru}(\mathrm{bpy}){ }_{3} \mathrm{Cl}_{2}(1 \mathrm{~mol} \%)$ & blue & $\mathrm{CH}_{3} \mathrm{CN}$ & $79 \%(6 a)$ \\
\hline
\end{tabular}




\begin{tabular}{|c|c|c|c|c|c|}
\hline 10 & $\begin{array}{l}1.2 \\
\text { equiv. }\end{array}$ & $\begin{array}{l}\left(\operatorname{Ir}\left[\mathrm{dF}\left(\mathrm{CF}_{3}\right) \mathrm{ppy}\right]_{2}(\mathrm{dtbpy})\right) \mathrm{PF}_{6} \\
\text { mol\%) }\end{array}$ & blue & $\mathrm{CH}_{3} \mathrm{CN}$ & $70 \%(6 a)$ \\
\hline 11 & $\begin{array}{l}1.2 \\
\text { equiv. }\end{array}$ & Eosin $Y(5 \mathrm{~mol} \%)$ & green & $\mathrm{CH}_{3} \mathrm{CN}$ & $23 \%(6 a)$ \\
\hline 12 & $\begin{array}{l}1.2 \\
\text { equiv. }\end{array}$ & 4CzIPN (5 mol\%) & blue & $\mathrm{CH}_{3} \mathrm{CN}$ & $60 \%(6 a)$ \\
\hline 13 & $\begin{array}{l}1.6 \\
\text { equiv. }\end{array}$ & 4CzIPN (5 mol\%) & blue & $\mathrm{CH}_{3} \mathrm{CN}$ & $77 \%(74 \%)^{e}(6 a)$ \\
\hline 14 & 2 equiv. & 4CzIPN (5 mol\%) & blue & $\mathrm{CH}_{3} \mathrm{CN}$ & $79 \%(6 a)$ \\
\hline 15 & $\begin{array}{l}2.5 \\
\text { equiv. }\end{array}$ & 4CzIPN (5 mol\%) & blue & $\mathrm{CH}_{3} \mathrm{CN}$ & $84 \%(6 a)$ \\
\hline 16 & $\begin{array}{l}1.6 \\
\text { equiv. }\end{array}$ & 4CzIPN (2.5 mol\%) & blue & $\mathrm{CH}_{3} \mathrm{CN}$ & $47 \%(6 a)$ \\
\hline 17 & $\begin{array}{l}1.2 \\
\text { equiv. }\end{array}$ & - & green & $\mathrm{CH}_{3} \mathrm{CN}$ & no conversion ${ }^{d}$ \\
\hline 18 & $\begin{array}{l}1.2 \\
\text { equiv. }\end{array}$ & $\mathrm{Cu}(\mathrm{dap})_{2} \mathrm{Cl}(0.5 \mathrm{~mol} \%)$ & - & $\mathrm{CH}_{3} \mathrm{CN}$ & no conversion \\
\hline 19 & $\begin{array}{l}1.6 \\
\text { equiv. }\end{array}$ & - & blue & $\mathrm{CH}_{3} \mathrm{CN}$ & no conversion \\
\hline 20 & $\begin{array}{l}1.6 \\
\text { equiv. }\end{array}$ & 4CzIPN (5 mol\%) & - & $\mathrm{CH}_{3} \mathrm{CN}$ & no conversion \\
\hline
\end{tabular}

${ }^{a}$ Reactions carried out on $0.1 \mathrm{mmol}$ scale. ${ }^{\mathrm{b}}$ Isolated yield. ${ }^{\mathrm{c}}$ Reaction carried out on $1 \mathrm{mmol}$ scale, 16 hours reaction time. d Partial cleavage of TMS group. ${ }^{\text {e }}$ Reaction carried out on $1 \mathrm{mmol}$ scale; 24 hours reaction time.

\section{General procedure for the optimization:}

The solvent $(0.5 \mathrm{~mL}, 0.2 \mathrm{M})$ was added to a mixture of $4 \mathbf{4 a}(0.1 \mathrm{mmol}), \mathrm{ABZ}(2,1.2-2.5$ equiv.) and the catalyst $(0.5-5 \mathrm{~mol} \%)$. The mixture was degassed ( 3 pump and freeze cycles) and then stirred for 16 hours at room temperature with irradiation of the indicated LED source. Thereafter the solvent was evaporated and the residue was purified by column chromatography (silica, pentane:EtOAc 30:1) affording 6a as a colorless oil. 
6. Ring expansion reactions

General Procedure E:

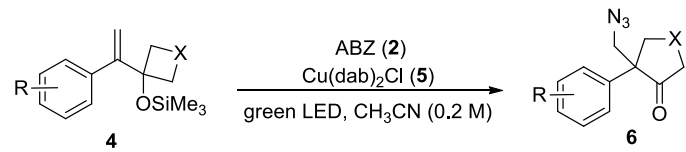

7. Product functionalization

2-Phenyl-2-((4-phenyl-1H-1,2,3-triazol-1-yl)methyl)cyclopentanone (27).

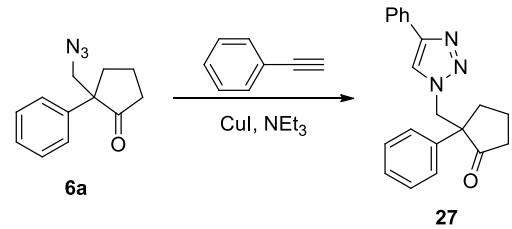

Benzyl ((2-oxo-1-phenylcyclopentyl)methyl)carbamate (28).

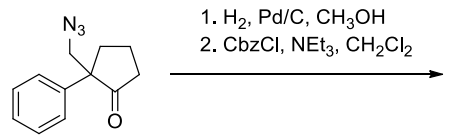

$6 a$

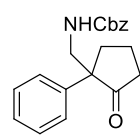

28

2-(Isothiocyanatomethyl)-2-phenylcyclopentanone (29).

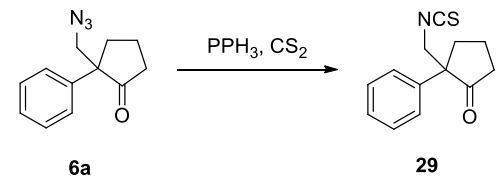

9. Established azidation reactions performed with tBuABX (1d) and ABZ (2)

9.1 Preparation of starting material for the azidation reactions

4-phenylpent-4-enoic acid (8)

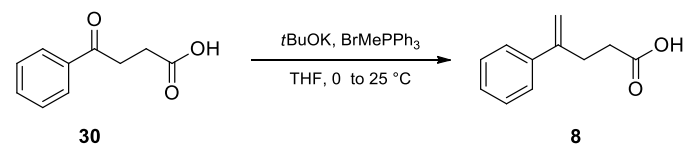

N-Phenyl-N-((2-vinylphenyl)sulfonyl)methacrylamide (10)

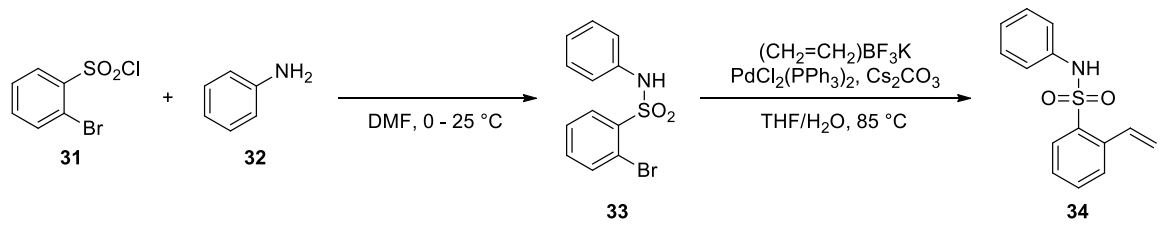

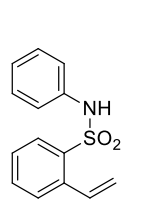

34

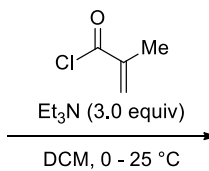

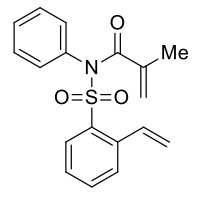


2-Allyl-N-methoxybenzamide (16)

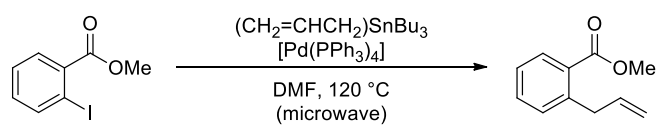

35

36

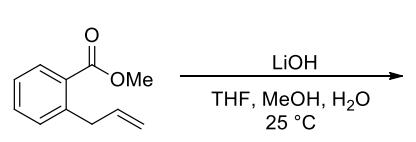

36

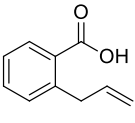

$\mathrm{H}_{2} \mathrm{NOMe} \cdot \mathrm{HCl}$ DMAP, EDC

37

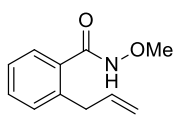

\subsection{Azidation experiments}

$\mathrm{Cu}(\mathrm{I})$-Catalyzed intramolecular oxyazidation of alkenes: Synthesis of 5-(azidomethyl)-5phenyldihydrofuran-2(3H)-one (9)

Experiment with ABX (1a)
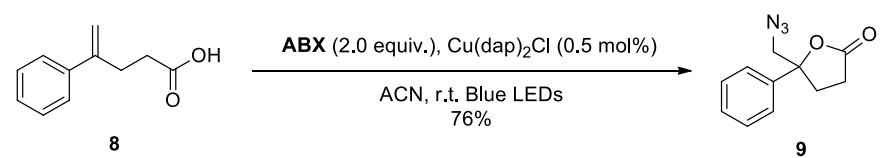

Experiment with $t B u A B X(1 d)$
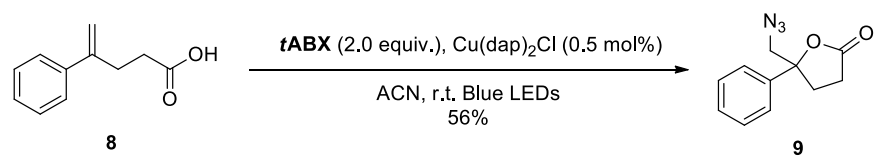

Experiment with ABZ (2)
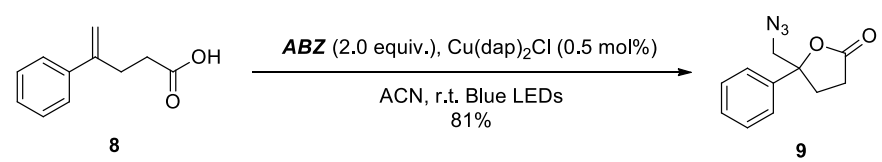

Generation of functionalized indanes through a complex radical cascade reaction: Synthesis of 3-(azidomethyl)-1-methyl-N-phenyl-2,3-dihydro-1H-indene-1-carboxamide (11)

Experiment with $t B u A B X(1 d)$

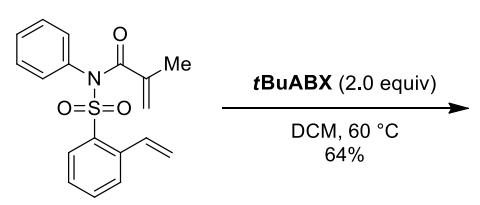

10

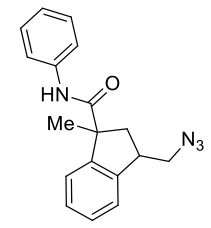

11

Experiment with ABZ (2)

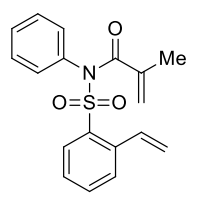

10
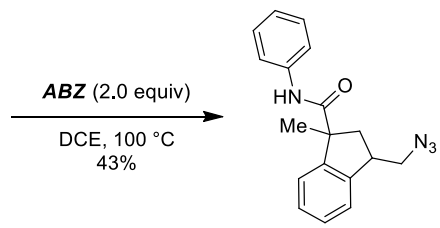

11 
Metal-catalysed azidation of tertiary C-H bonds: Synthesis of (2-azidopropan-2-yl)benzene (23)

\section{Experiment with ABX (1a)}
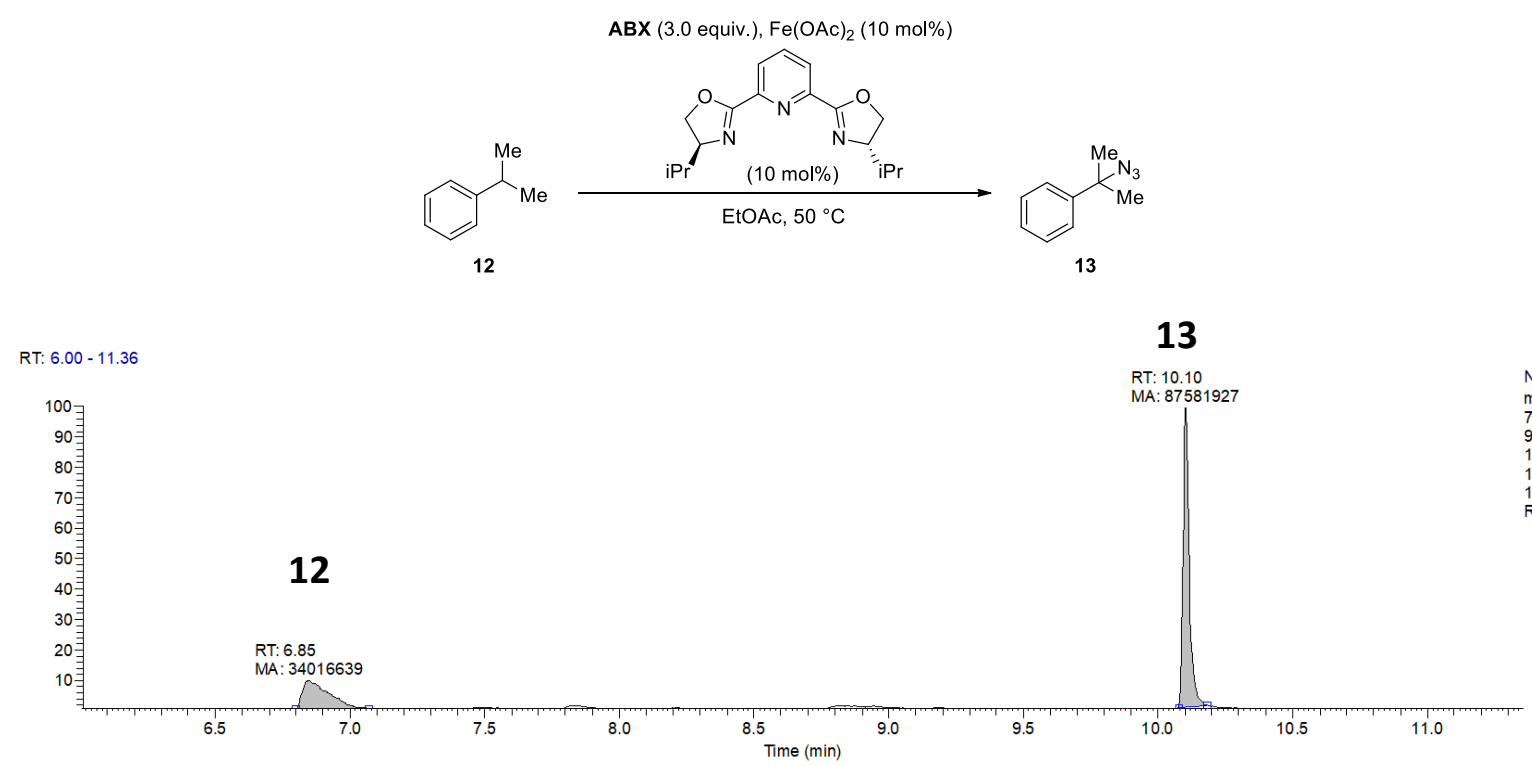

Figure S1: GC-MS chromatogram showing the conversion of cumene (12) into (2-azidopropan2-yl)benzene (13) after 22 hours when ABX (1a) was used.

\section{Experiment with $t B u A B X(1 d)$}

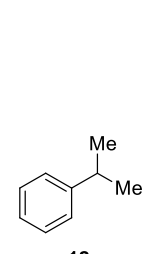

12

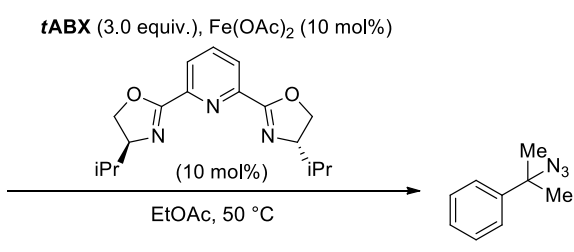

13

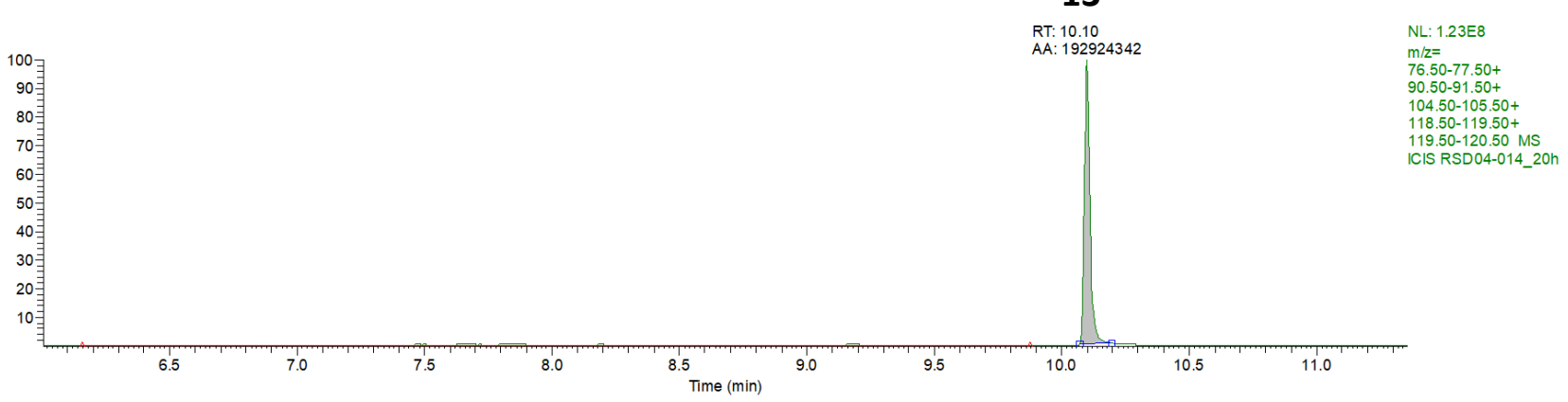

Figure S2: GC-MS chromatogram showing the conversion of cumene (12) into (2-azidopropan2-yl)benzene (13) after 22 hours when $t B u A B X(1 d)$ was used.

\section{Experiment with ABZ (2)}

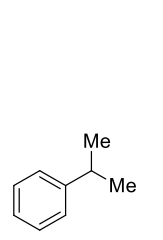

12

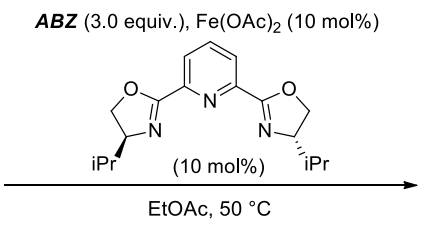

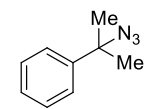

13 


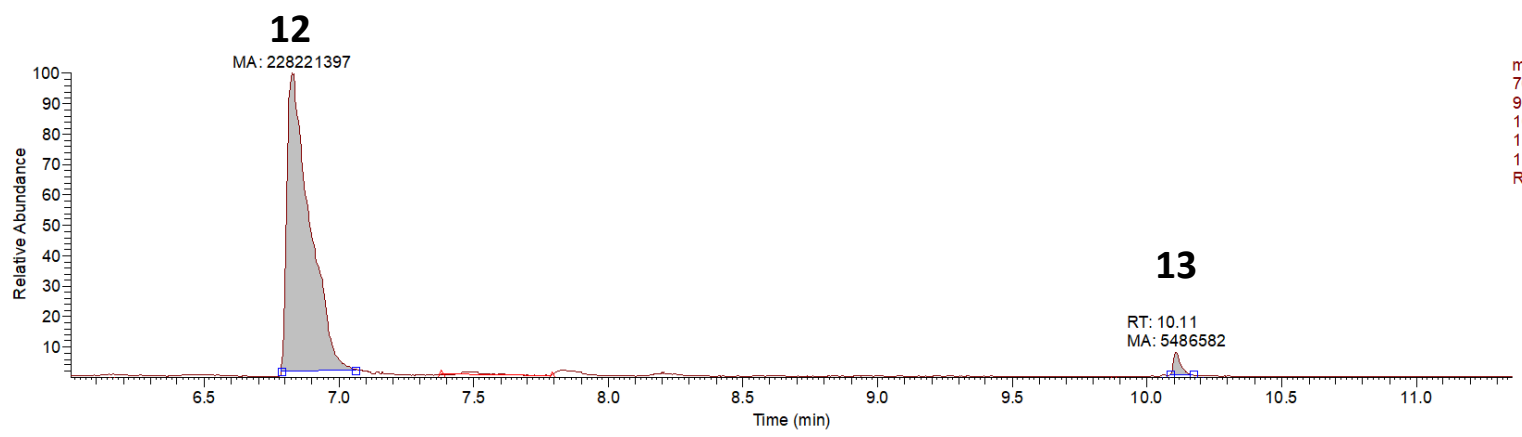

Figure S3: GC-MS chromatogram showing the conversion of cumene (12) into (2-azidopropan2-yl)benzene (13) after 22 hours when ABZ (2) was used.

$\mathrm{Cu}(\mathrm{II})$-Catalyzed azidation of anilines: Synthesis of 2-azido-4-methylaniline (15)

Experiment with ABX (1a)

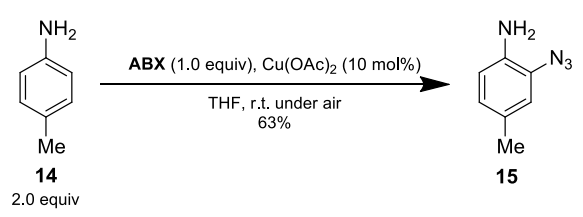

Experiment with $t B u A B X(1 d)$

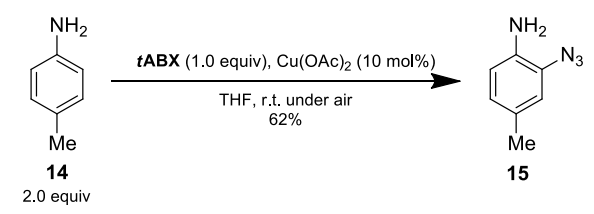

Experiment with ABZ (2)

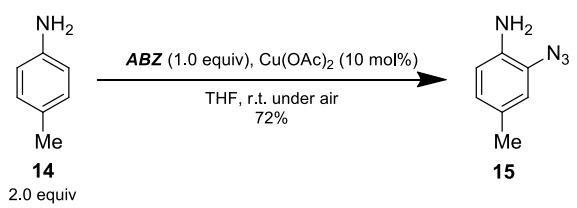

$\mathrm{Cu}(\mathrm{I})$-Catalyzed intramolecular alkene aminoazidation: Synthesis of 3-(azidomethyl)-2methoxy-3,4-dihydroisoquinolin-1(2H)-one (17)

Experiment with $t B u A B X(1 d)$

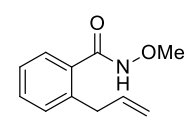

16 $\begin{gathered}\text { tBuABX }(2.0 \text { equiv) } \\ \text { CuOAc }(10 \mathrm{~mol} \%)\end{gathered}$
$\underset{\mathrm{MeCN}, 60^{\circ} \mathrm{C}}{73 \%}$

(1)

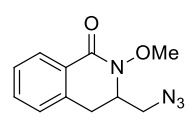

17

Experiment with $A B Z(2)$

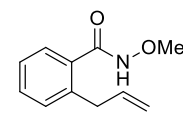

16

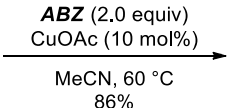
$86 \%$

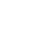




\section{Accident report and safety studies}

Before the accident: two detonations on small scale (20-30 $\mathrm{mg}$ ) were observed when adding the compound to a flask containing an organocatalyst and a substrate (both solids). The compound is shock sensitive as demonstrated by hammer test.

\section{Accident report}

\section{Followed experimental protocol}

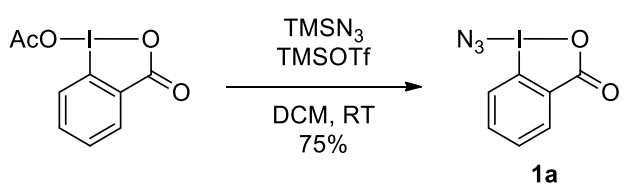

Caution: reaction carried out behind a safety shield! Following a reported procedure, the starting material ( $1.00 \mathrm{~g}, 3.28 \mathrm{mmol}, 1.00$ equiv, synthesized in our laboratory) was stirred in dry DCM ( $3 \mathrm{~mL}$, dried by passage over activated alumina under nitrogen atmosphere) in a thoroughly washed round-bottom glass flask, then $\mathrm{TMSN}_{3}(0.66 \mathrm{~mL}, 4.9 \mathrm{mmol}, 1.5$ equiv, $94 \%$ from Alfa Aesar, catalogue number L00173-22) was cautiously added. A catalytic amount of TMSOTf ( $3 \mu \mathrm{L}, 0.02 \mathrm{mmol}, 0.005$ equiv, Fluorochem, catalogue number S20400-250g) was added last to the mixture which was then stirred for 30 minutes. The solvent was then removed under reduced pressure at room temperature and the residue was dried in vacuo for one hour to give a yellow solid, which was washed with pentane $(2 \times 10 \mathrm{~mL})$, cold acetone $(2 \times 5$ $\mathrm{mL})$ and pentane $(2 \times 10 \mathrm{~mL})$ and dried one hour in high vacuo. For each wash, the following procedure was followed: add solvent, stir 5 min, filtered on a frit, breaking larger pieces with metal spatula, and remove solvent during one minute on the frit. Usual yield around $70 \%$ (not determined on this batch due to the accident, see below).

Note: this is the reported method 2 in EROS:

http://onlinelibrary.wiley.com/doi/10.1002/047084289X.rn02053/abstract?selectedXmlld=r n02053-eo-c00022\&userlsAuthenticated $=$ false \&deniedAccessCustomisedMessage $=$ With the difference of smaller reaction scale and the solvent used for washing (hexane was used in EROS). Acetone was found to be more efficient for removing yellow impurity in the compound.

\section{Accident:}

On the day of the experiment, the researcher (experienced postdoc having done already 200 experiments with the compound without any accident) wanted to use the batch immediately after preparing it to have the best quality possible. In the previous experiments, he had just filled the flask with nitrogen and stored the compound in the fridge for several weeks. This time, after drying $1 \mathrm{~h}$ in vacuum, he filled the flask with air and remove it from the vacuum line to weight it on the balance. The compound was first scratched from the wall of the flask with the spatula and then he slightly shook the flask (with the spatula in) to collect the solid at the bottom. At this point, it detonated. The flask was pulverized. As it was open, most of the energy went fortunately upwards. 


\section{Consequences:}

The left hand had multiple blooding small cuts and burns (1. Degree and 2. Degree on the thumb). Minor cuts also on the face and between face and chest (areas not protected by the lab coat). The safety glasses protected the eyes. Hearing was imparted through the detonation.

Immediate treatment at the emergency room of the local hospital followed. It was decided not to remove the glass fragments, as they were very small and should be resorbed naturally. If not the case, an operation may be needed later. Hearing problems, high noise sensitivity and headaches slowly decreased over two weeks. Work incapacity for two weeks. At this point, no long-term damage expected.

\section{Analysis:}

The cause of explosion could not be identified. Ongoing hypotheses:

- Remaining impurities: HN3? (but the wash was very thorough and careful)

- Explosive impurities formed with acetone (but intensive wash afterwards and no volatile should be left).

- Initiating non-volatile impurities, such as traces of metal

- The compound was pure, but the explosion was initiated by shock (very small one!) or electrostatic charging (via friction? indeed method of work not optimal in this case)

\section{Proposed measures:}

- Whenever possible, avoid using this compound!

- Diminish batch quantities (< $500 \mathrm{mg}$ starting material)

- All actions behind safety shield (including weighting) with enhanced protection (explosion resistant mask and gloves)

- Action against electrostatic and shock (in particular no metal spatula, possibly avoid glass?)

- Diminish explosion potential of reagent by structure or formulation modification.

It seems very difficult to identify surely the cause(s) of explosion, exactly the same steps having been done several times in the lab, therefore the way to go is probably to envisage chemical modifications by adding stabilizing additive or changing the structure of the reagent (diminish nitrogen to carbon ratio) to decrease its explosion potential.

\section{Safety Studies}

The stability of the compounds was determined by differential scanning calorimetry (DSC). A few $\mathrm{mg}$ of samples were sealed in gold plated pressure resistant crucibles. The crucibles were heated in an oven from $30^{\circ} \mathrm{C}$ to $400^{\circ} \mathrm{C}$ at a rate of $4^{\circ} \mathrm{C} / \mathrm{min}$. The Tonset (temperature at which an exothermic signal is detected), the decomposition energy and the peak temperature were recorded and compared. The provided data are the mean of two measurements. 
The sensitivity to friction and impact as well as the energy of decomposition measured by DSC were determined for the Zhdankin reactive (1a) as well as for tBu-ABX (1d) and $A B Z(2)$.

The sensitivity to impact or shock (Fallhammer test) consists of subjecting 40 substance to different drop-weight impact. The impact energy is varied by the selection of drop height and weight. A drop-weight of $1 \mathrm{~kg}$ was used for this study. The test is positive if at least one positive result (detonation, fume, fire or sparks) in a series of six measurement is obtained. Here a microphone was used for the detection. The limiting impact energy is the lowest energy at which there is still a positive result. The tests were performed with $1 \mathrm{~J}$ steps.

The sensitivity to friction was measured with a BAM friction apparatus. A porcelain plate with $10 \mathrm{ul}$ of test substance was moved by an electric motor against a stationary porcelain peg. The load corresponded to a friction force of $360 \mathrm{~N}$. The test was then repeated on Alox (aluminium oxide) plates as many false positive are measured with porcelain plates.

11 Spectra of compounds synthesized by modified methods and of new compounds 
$\mathrm{ABX}(1 \mathrm{a})$
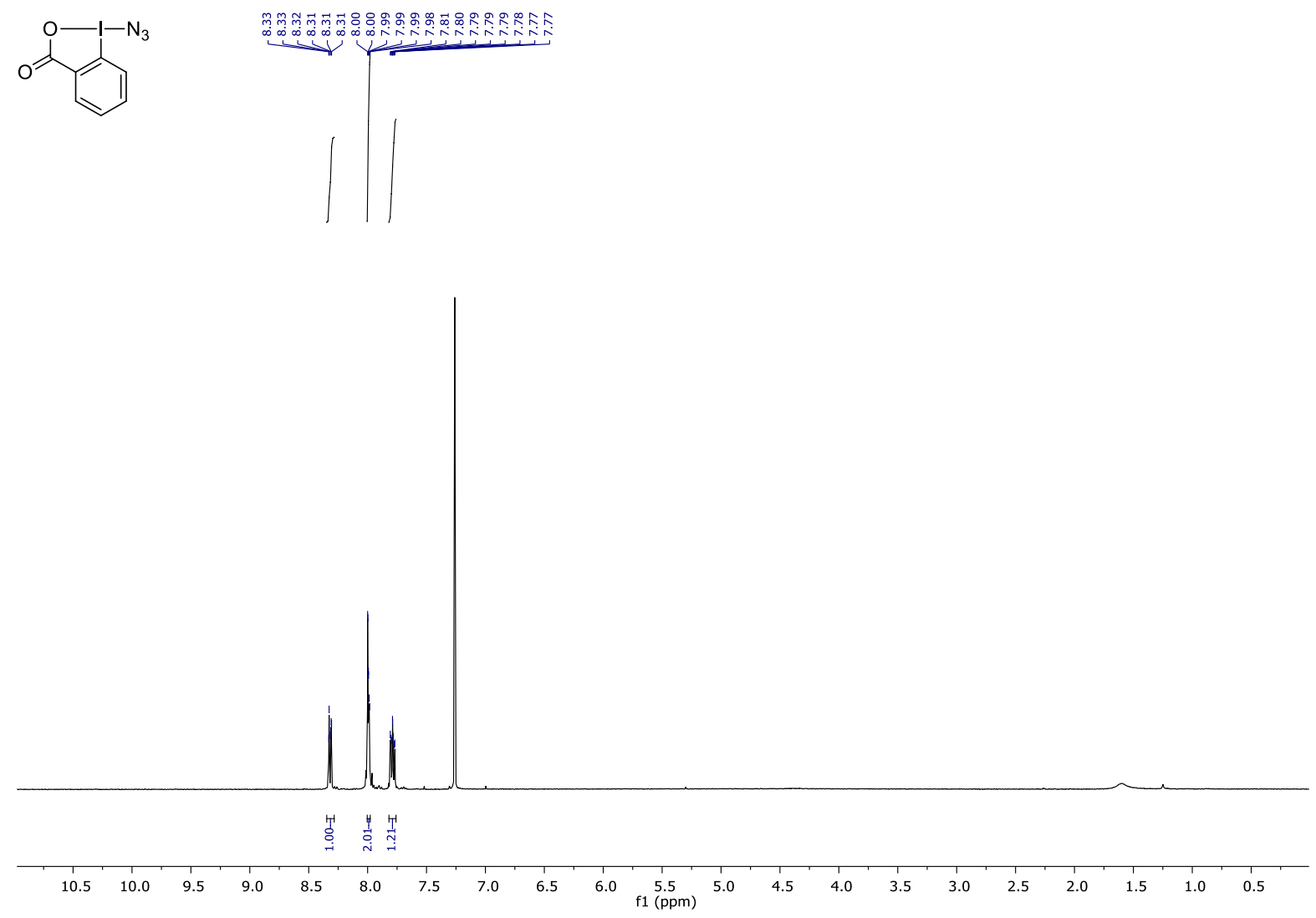

5-(Azidomethyl)-5-phenyldihydrofuran-2(3H)-one (9)

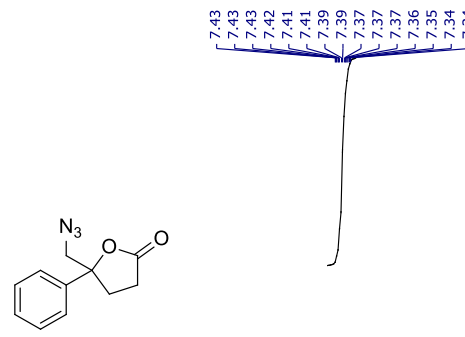

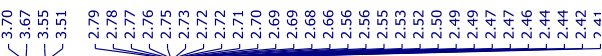
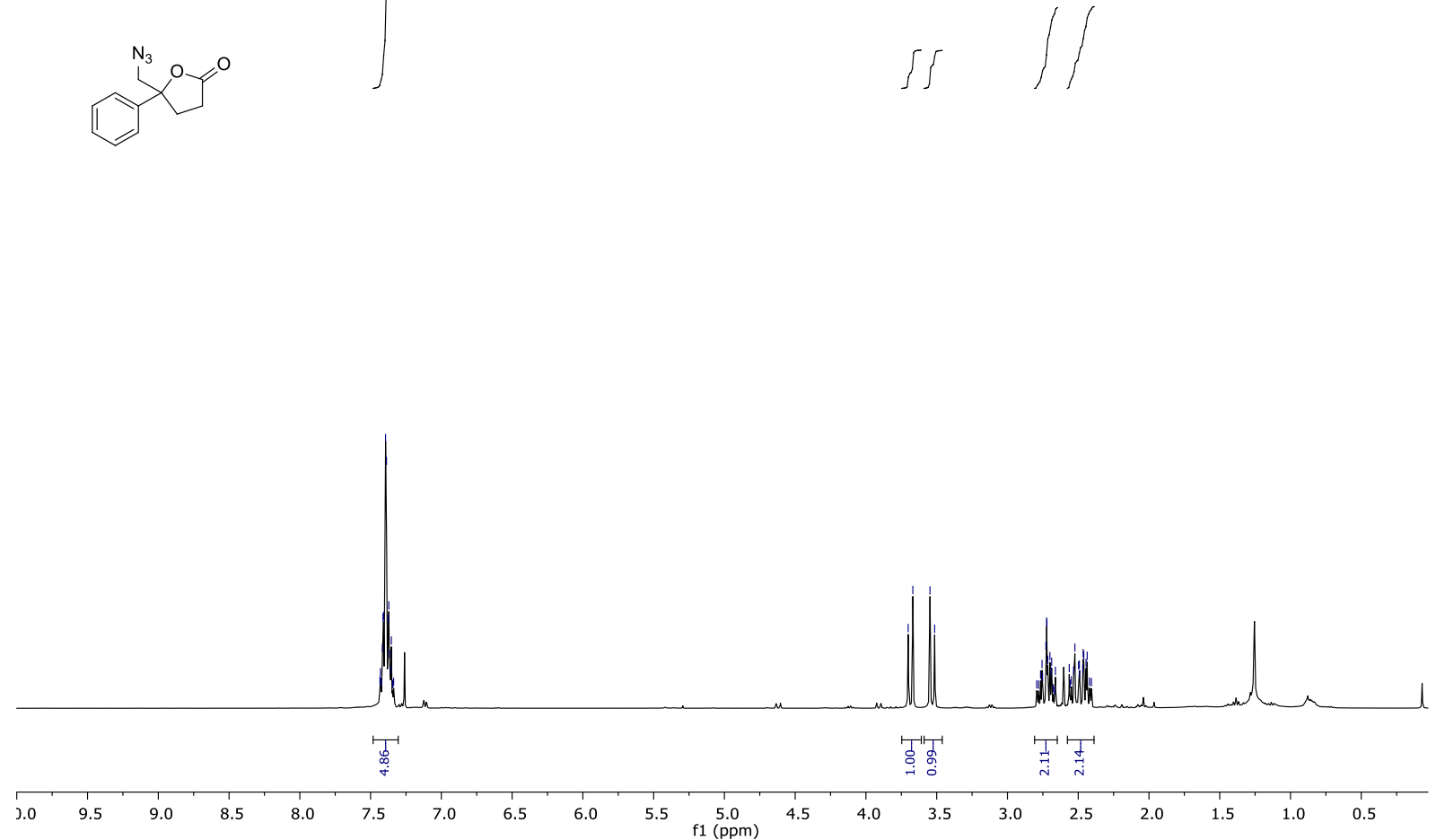
3-(Azidomethyl)-1-methyl-N-phenyl-2,3-dihydro-1H-indene-1-carboxamide (11)

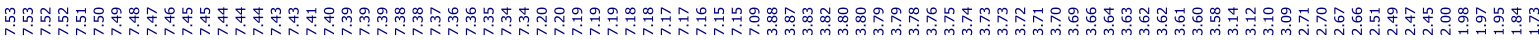

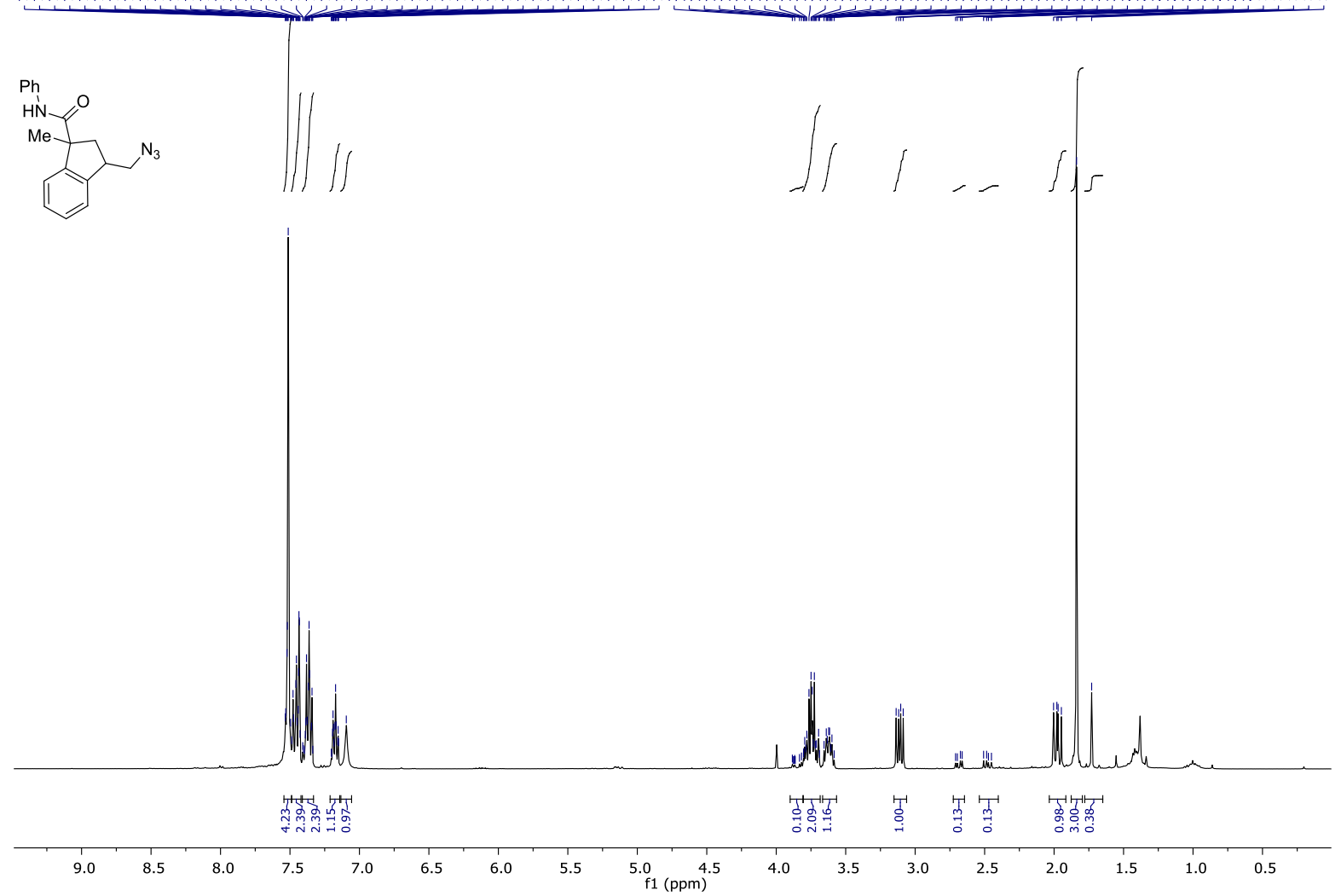

2-Azido-4-methylaniline (15)

$\underbrace{\mathrm{NH}_{3}}_{\mathrm{Me}}$

$\underbrace{200000}$

$\iint$
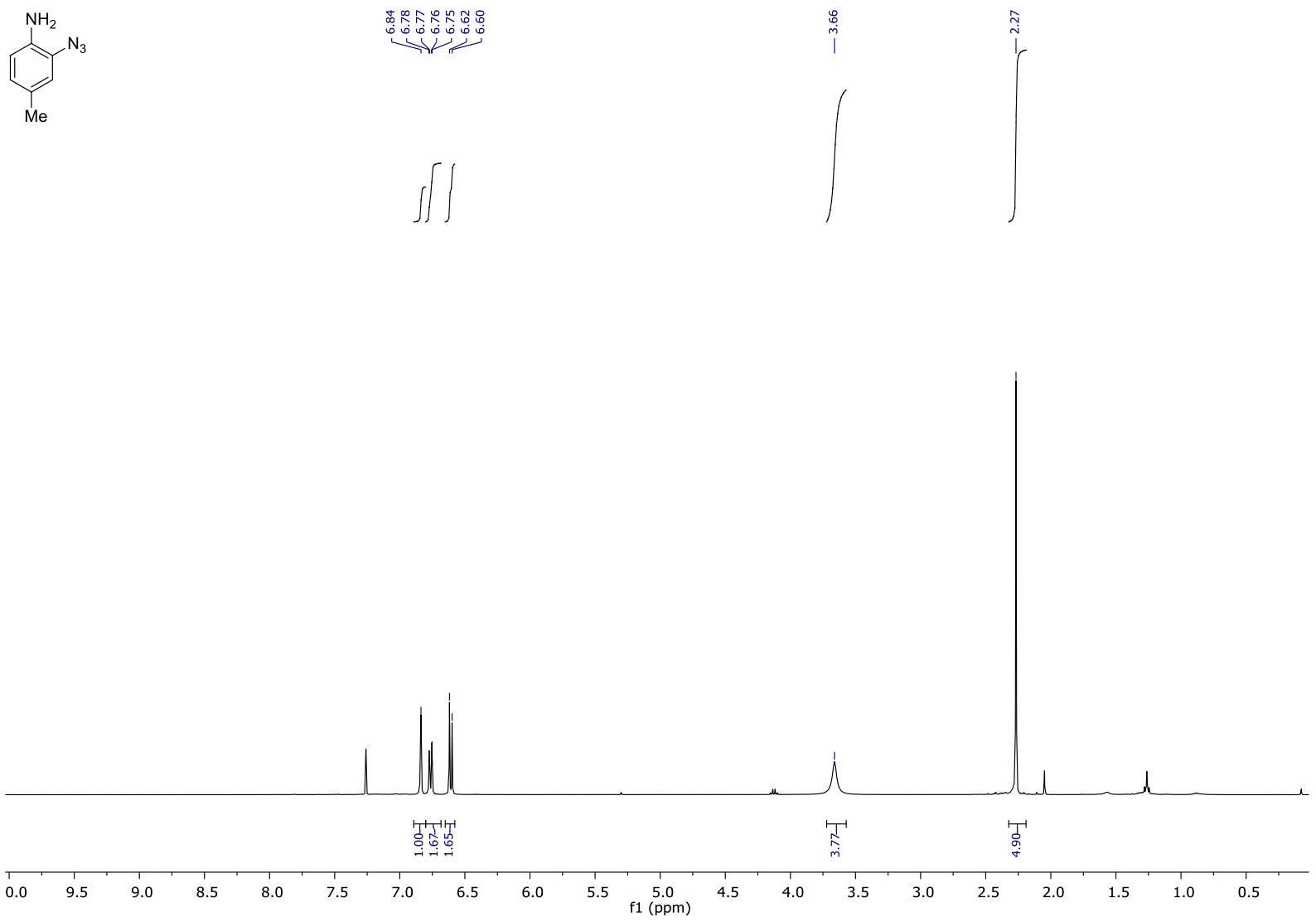
3-(Azidomethyl)-2-methoxy-3,4-dihydroisoquinolin-1(2H)-one (17)
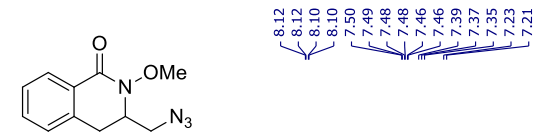

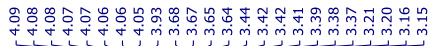

111
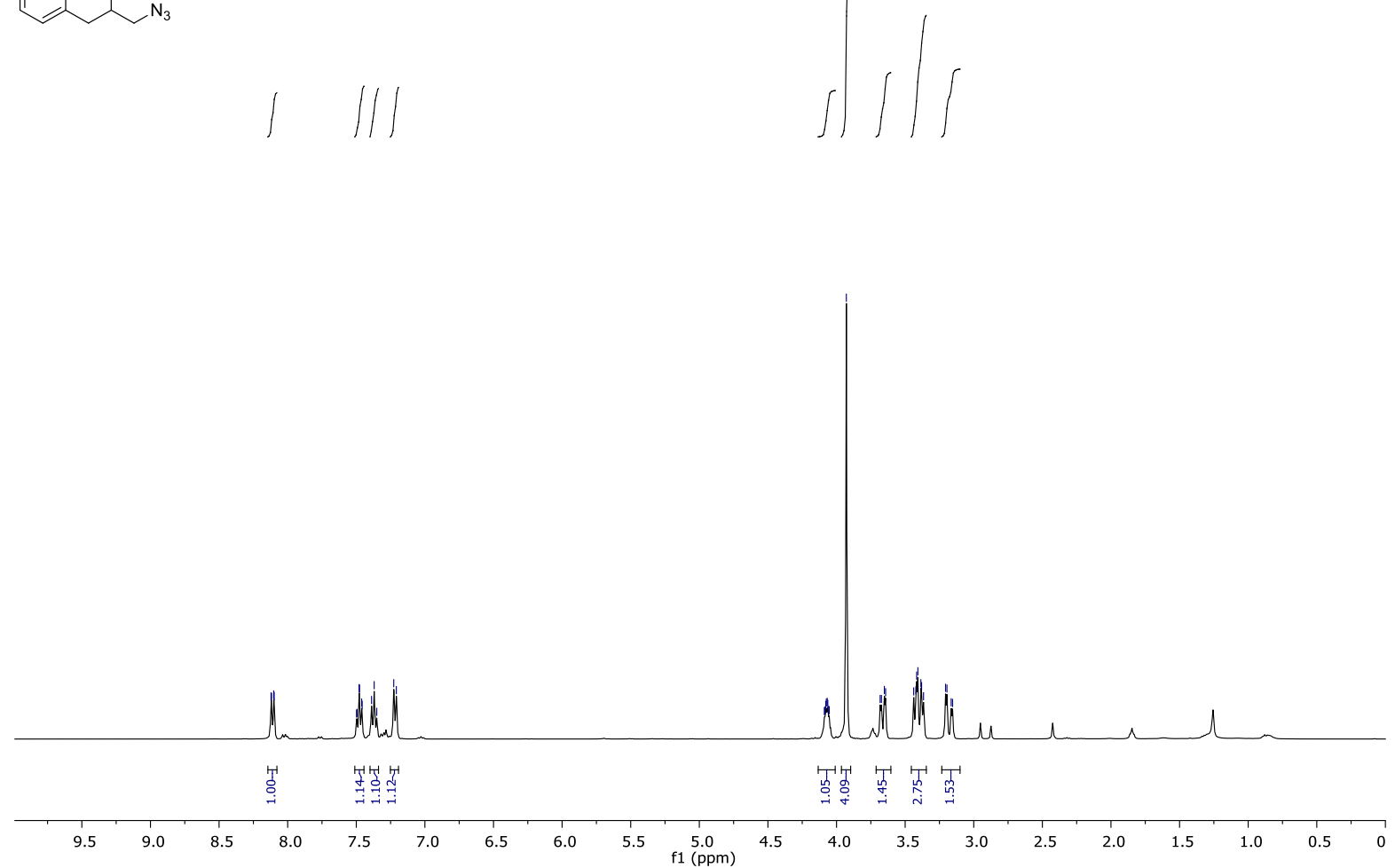
2-iodo- $N$-tosyl benzamide (31)

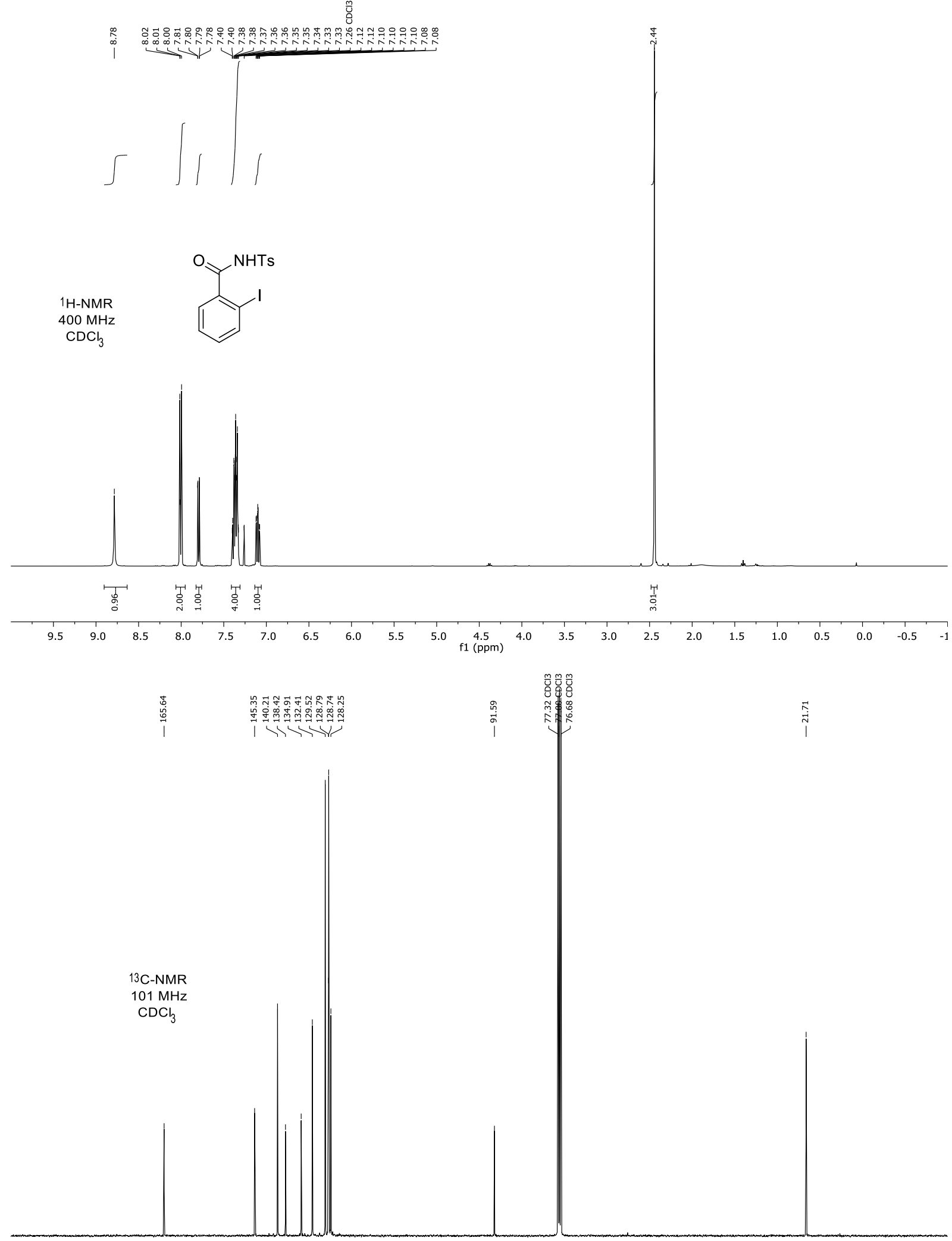

\begin{tabular}{llllllllllllllllllllll}
\hline 00 & 190 & 180 & 170 & 160 & 150 & 140 & 130 & 120 & 110 & $\underset{\mathrm{f} 1}{1}(\mathrm{ppm})$ & 80 & 70 & 60 & 50 & 40 & 30 & 20 & 10 & 0 & -
\end{tabular} 
Acetato ( $N$-tosyl)benziodazole (32).

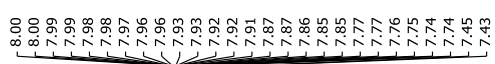

$\| 1$

1H-NMR

$400 \mathrm{MHz}$<smiles></smiles>

D6-DMSO

'́Ac

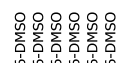

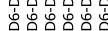

|

ity

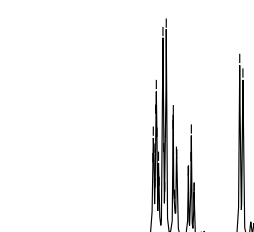

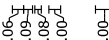

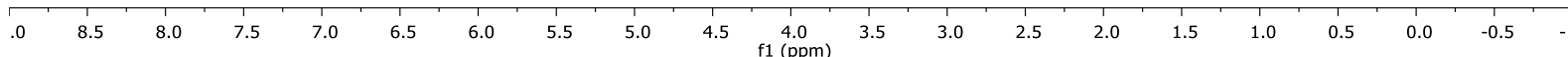

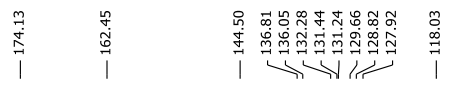

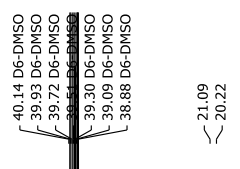

13C-NMR

$101 \mathrm{MHz}$

D6-DMSO
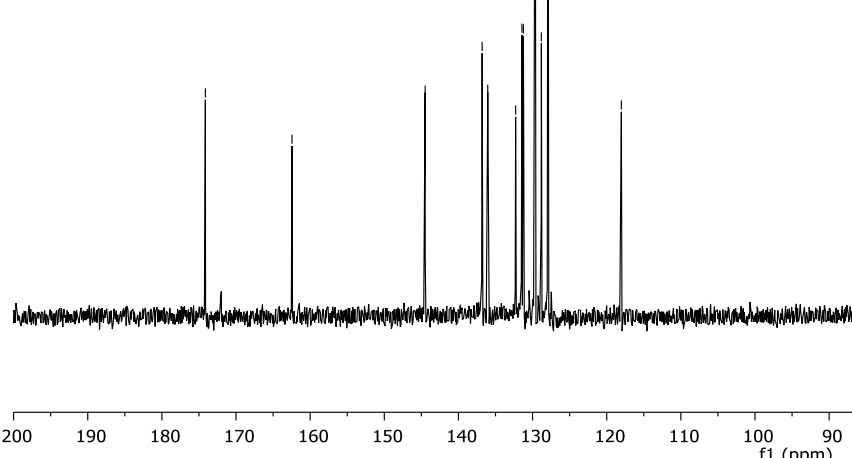

f1 (ppm) 
Azido (N-tosyl)benzoiodazole (2).

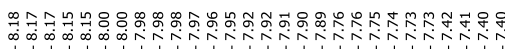<smiles>c1ccccc1</smiles>

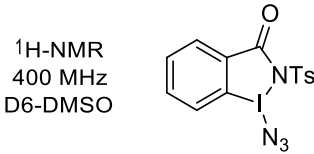

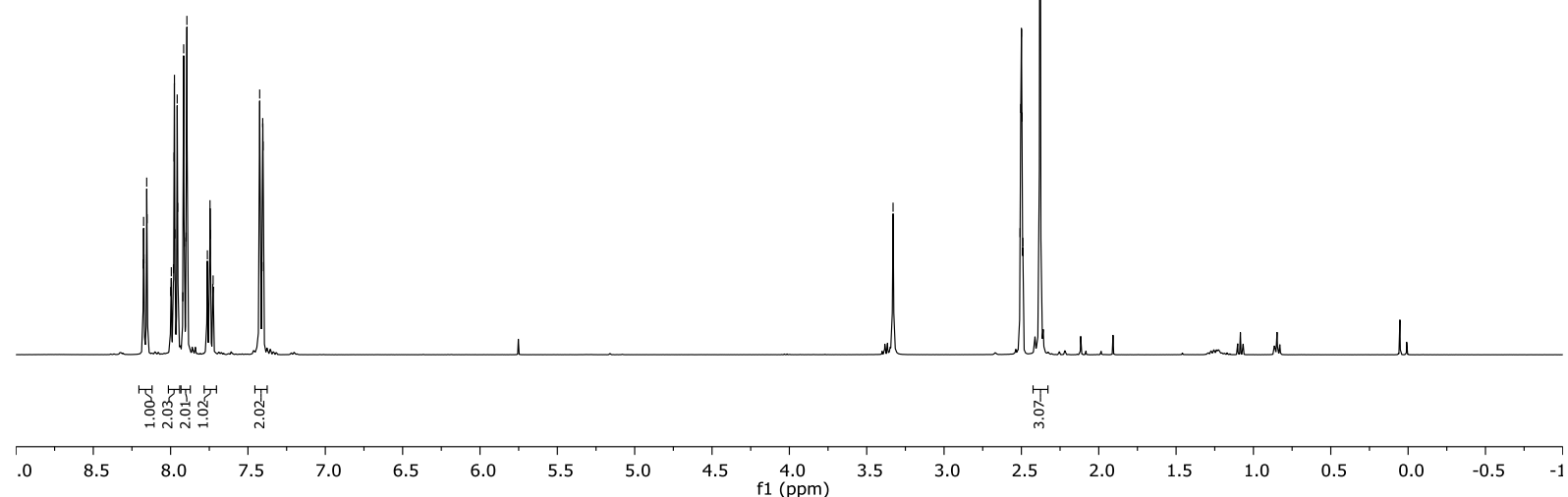

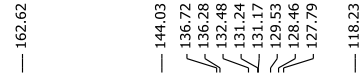

13C-NMR

$101 \mathrm{MHz}$

D6-DMSO

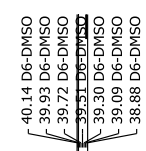

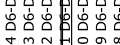

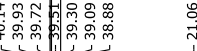

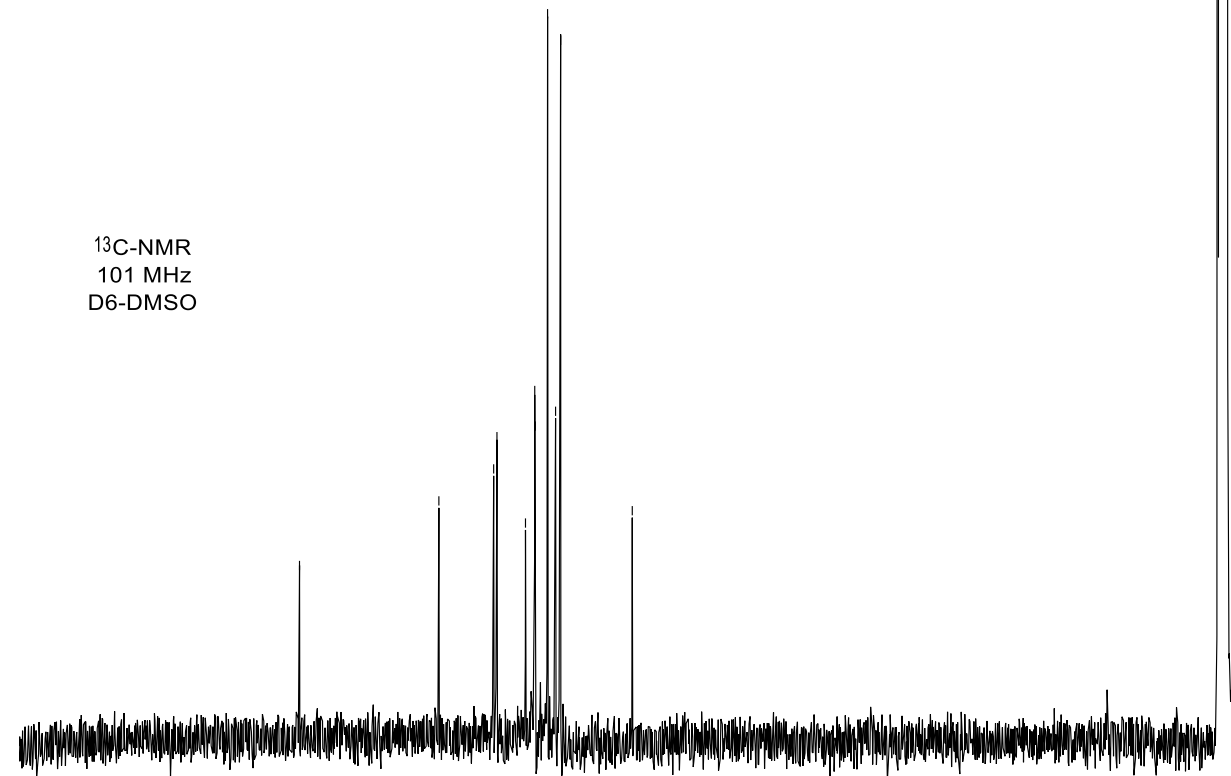

$1 \mathrm{H}-\mathrm{NMR}$

$\begin{array}{lllllllllll}00 & 190 & 180 & 170 & 160 & 150 & 140 & 130 & 120 & 110 & 100 \\ \mathrm{f} 1(\mathrm{ppm}) & 90\end{array}$

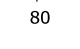

70

50

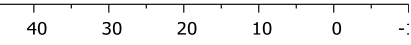


4-(1-Bromovinyl)-1,2-dimethylbenzene (33I).

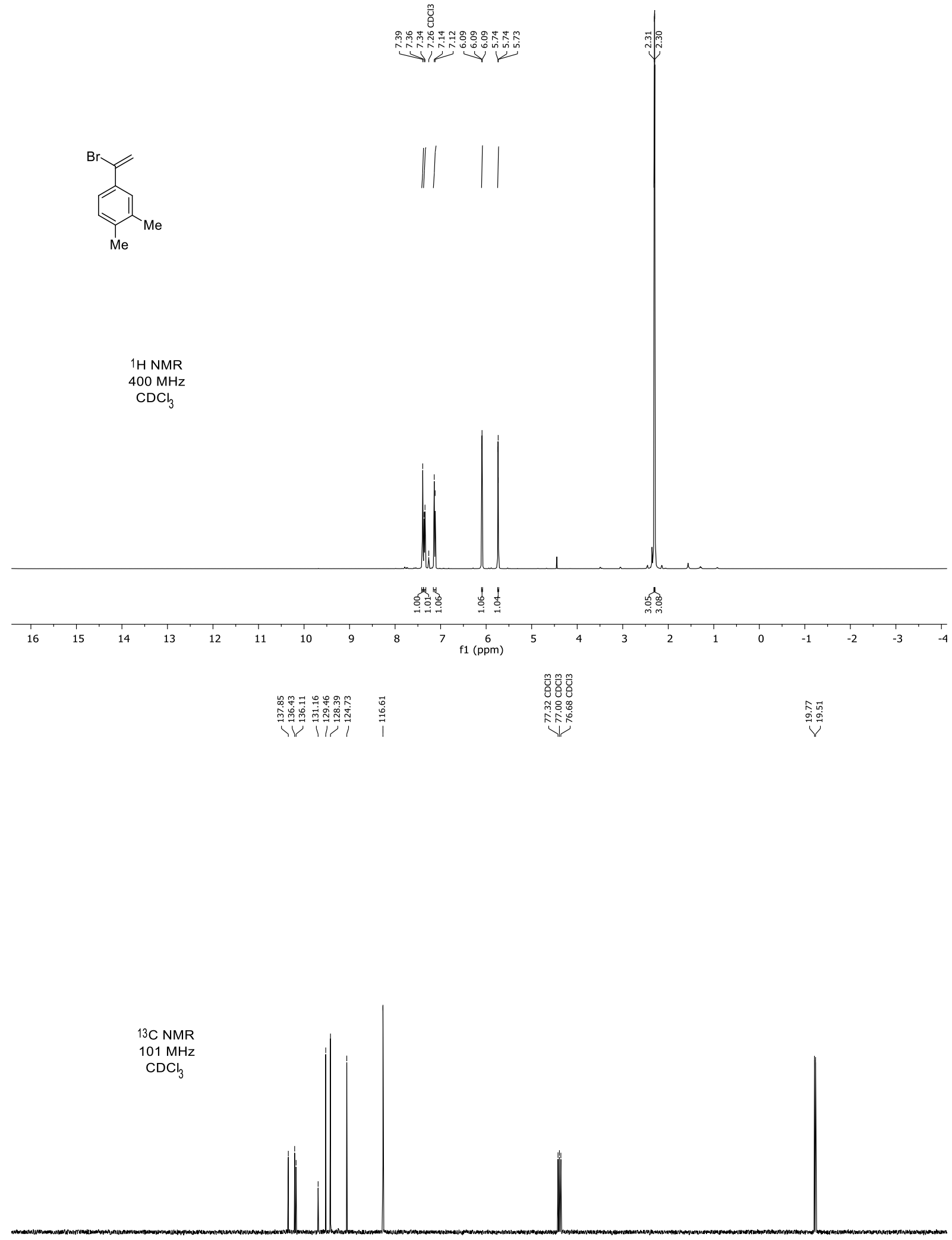

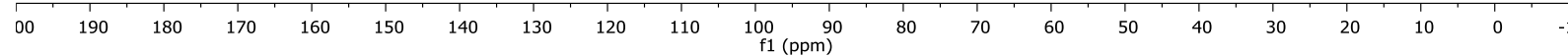


1-(1-Bromovinyl)-4-(trifluoromethyl)benzene (33t).
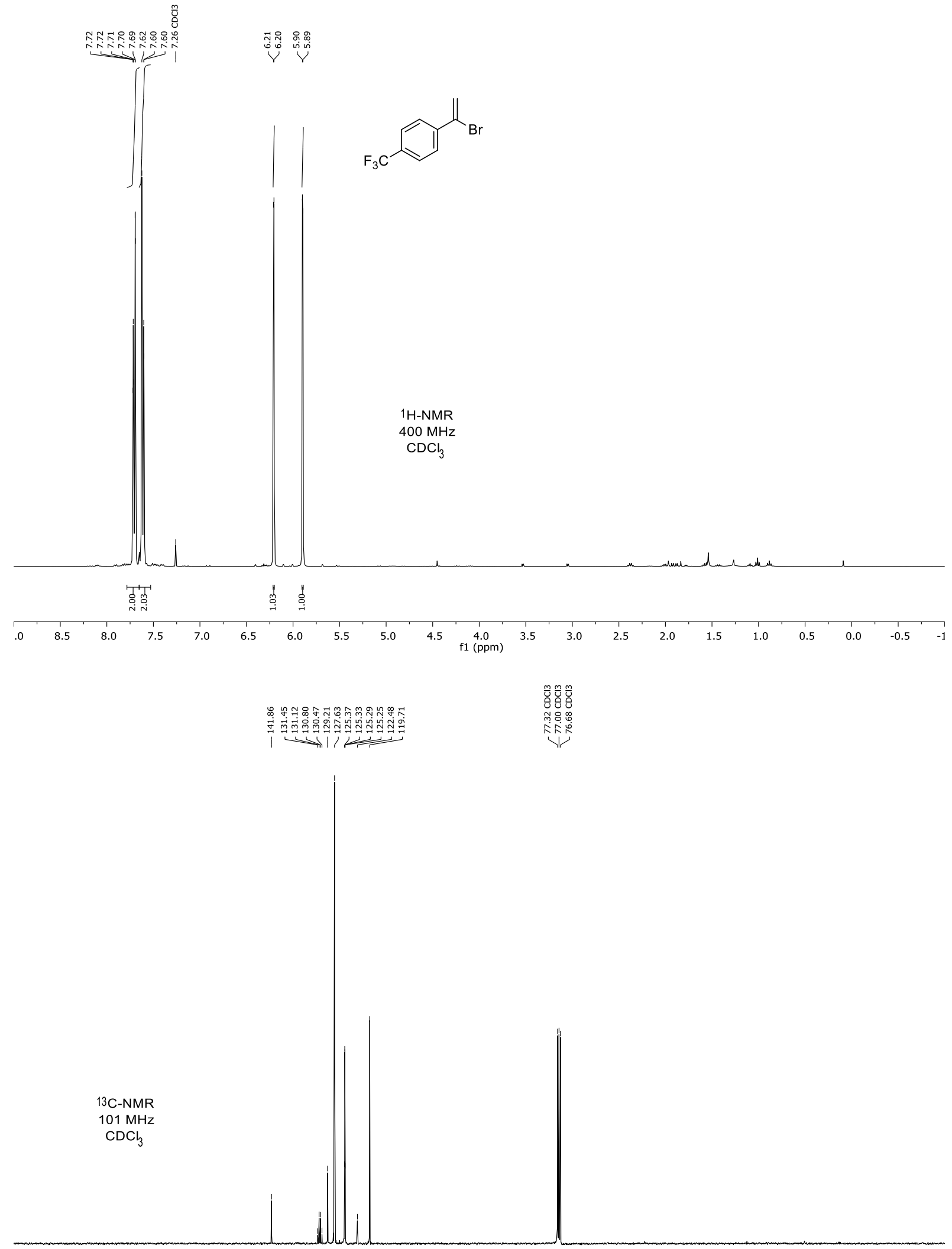

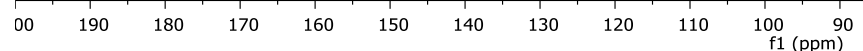

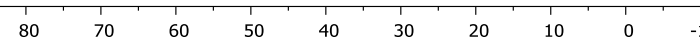


要

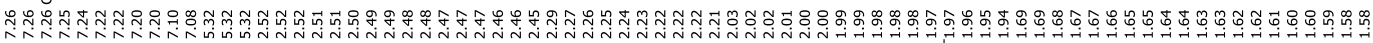

$\longrightarrow \longrightarrow$
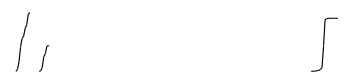

$\iint 1$

Me

H-NMR

$400 \mathrm{MHz}$

$\mathrm{CDCl}_{3}$

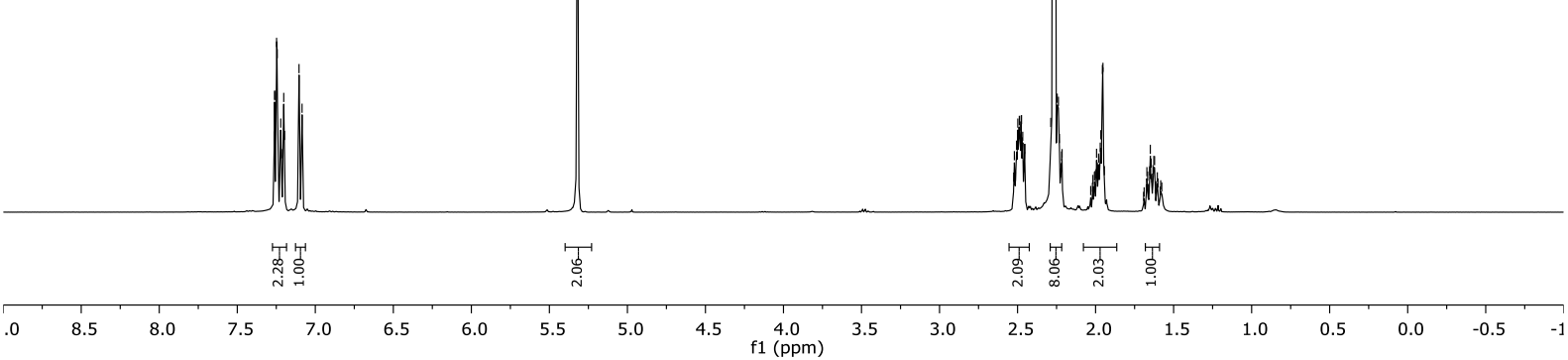


1-(1-(3,4-dimethoxyphenyl)vinyl)cyclobutanol (3m)

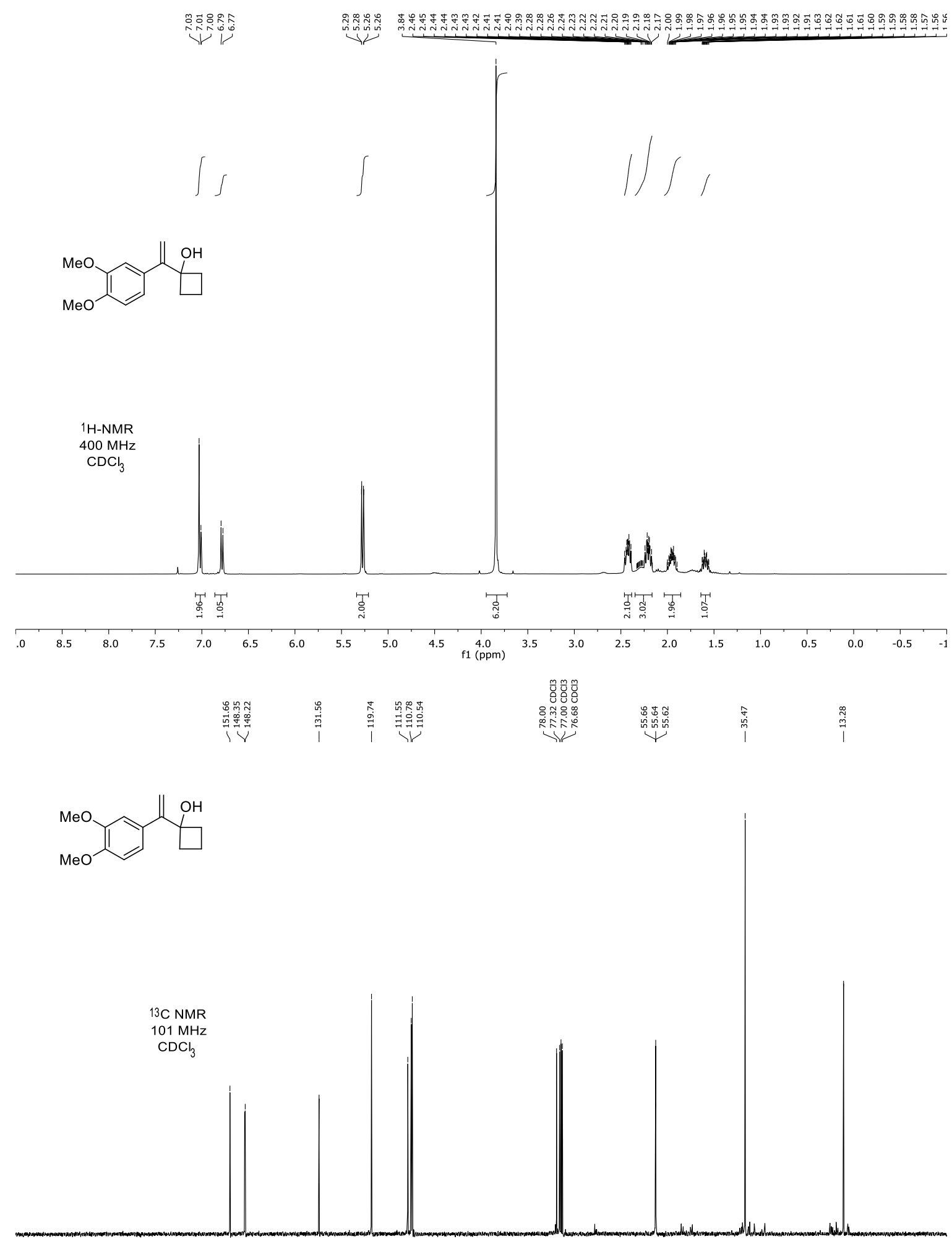

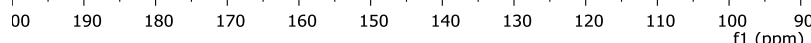


1-(1-(Naphthalen-1-yl)vinyl)cyclobutanol (3p).

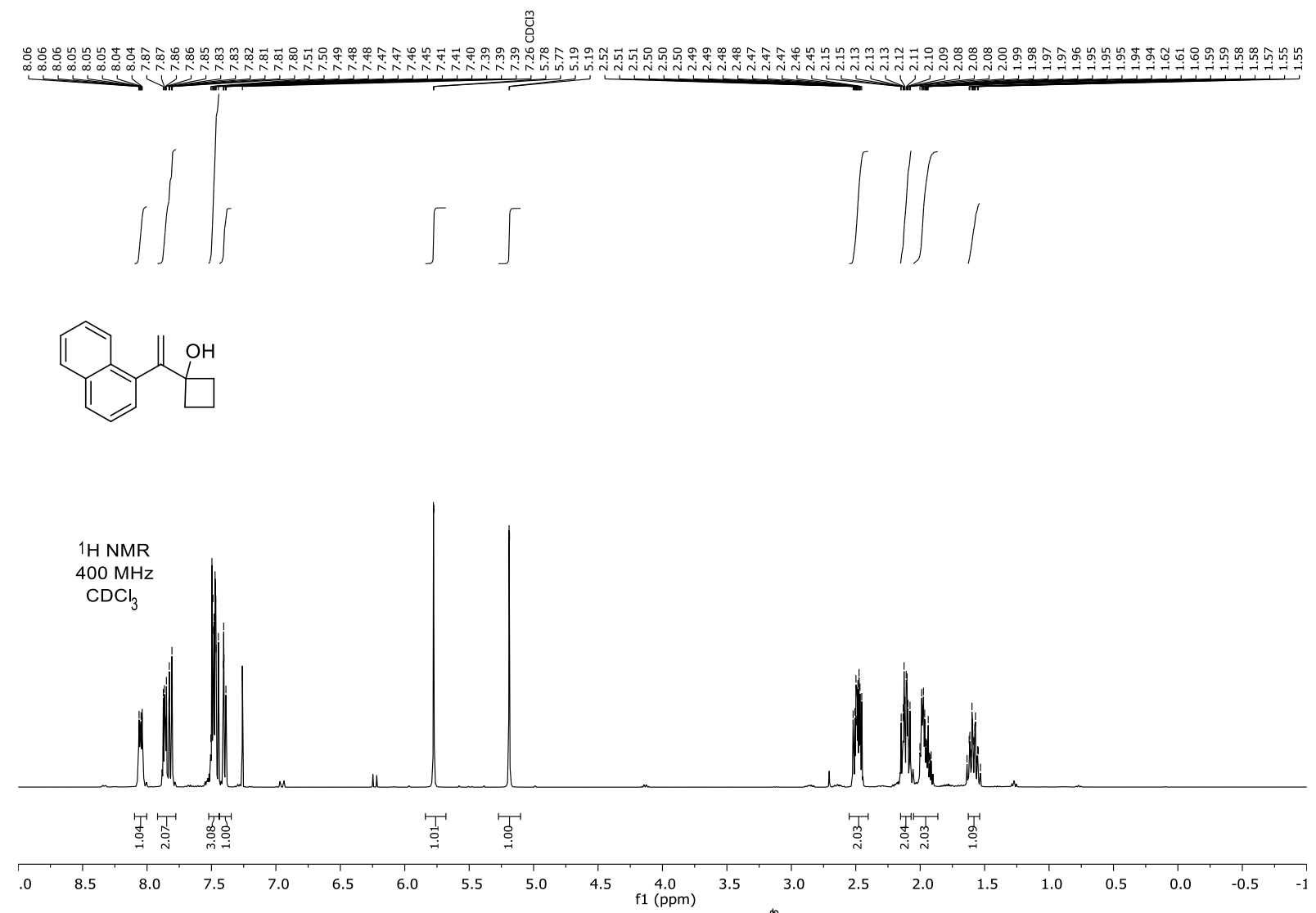
|

$101 \mathrm{MHz}$

$\mathrm{CDCl}_{3}$

\begin{tabular}{lllllllllll}
\hline 00 & 190 & 180 & 170 & 160 & 150 & 140 & 130 & 120 & 110 & $\begin{array}{c}100 \\
\mathrm{f} 1(\mathrm{ppm})\end{array}$
\end{tabular} 
tert-Butyl 3-hydroxy-3-(1-phenylvinyl)azetidine-1-carboxylate (3r)
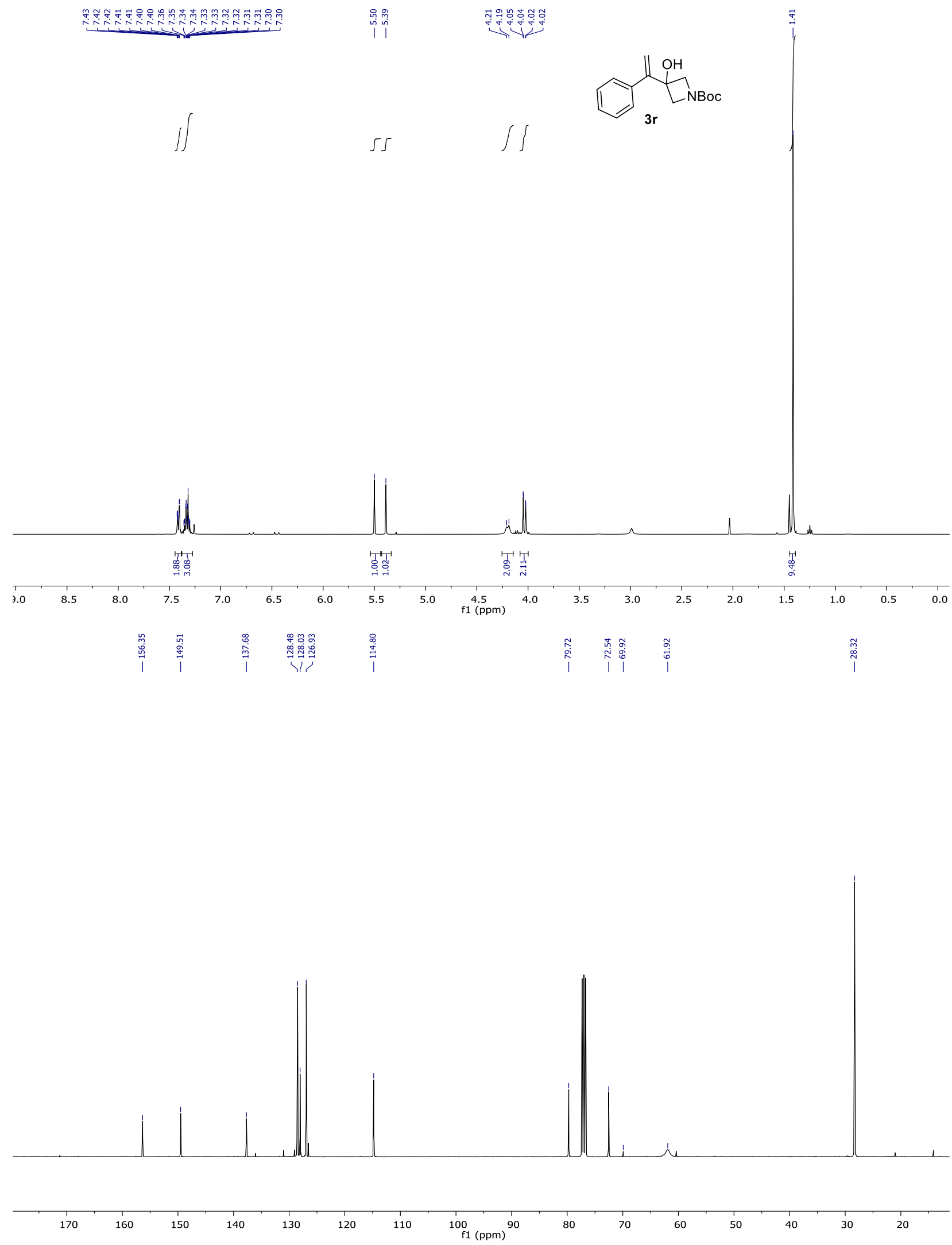
1-(1-(4-(Trifluoromethyl)phenyl)vinyl)cyclobutanol (3t).
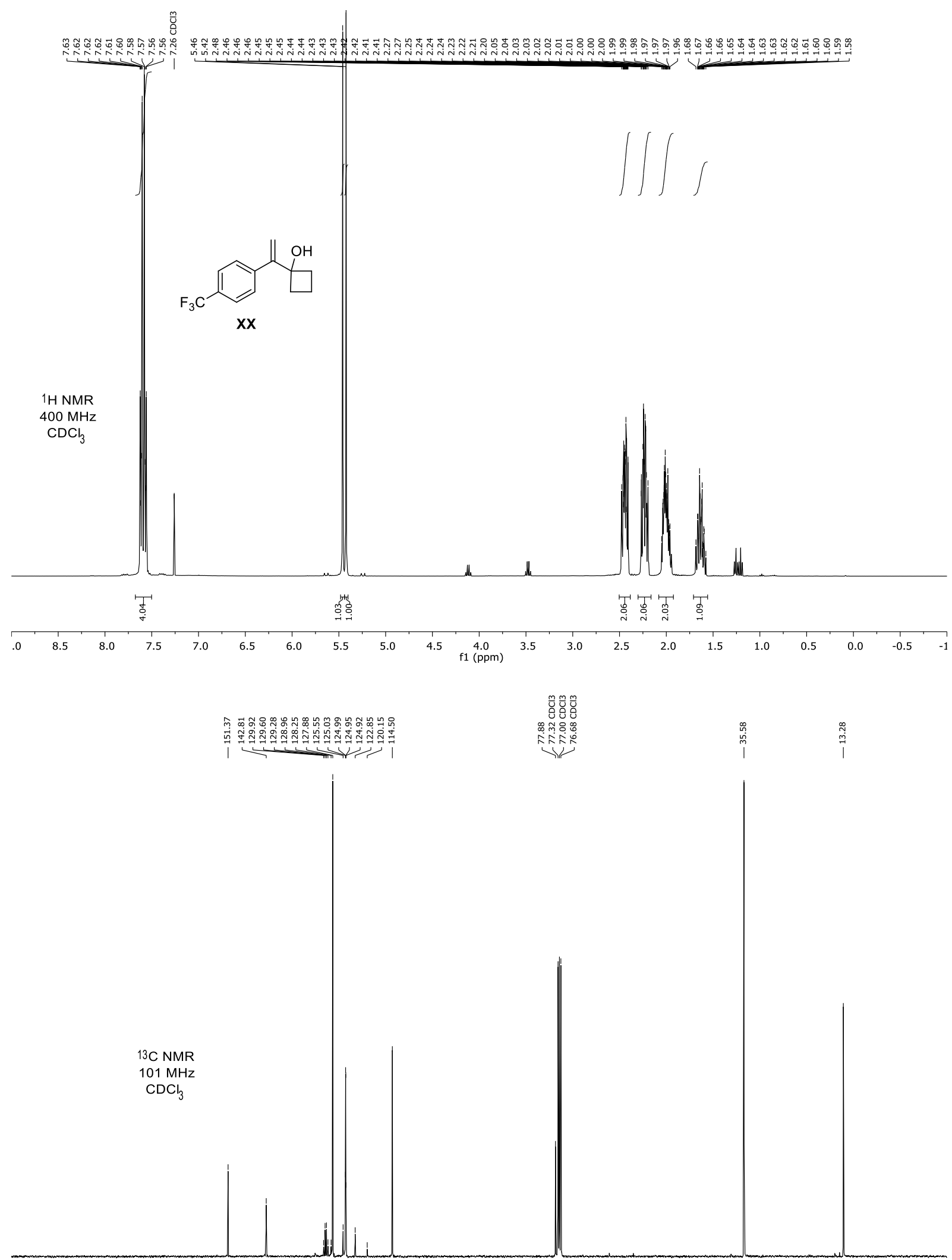

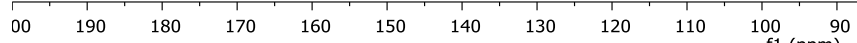


Trimethyl(1-(1-phenylvinyl)cyclobutoxy)silane (4a)

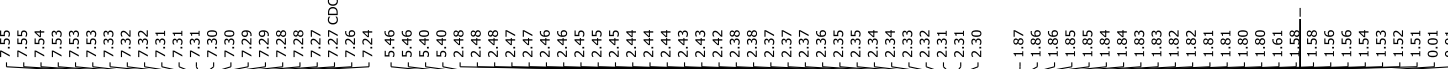

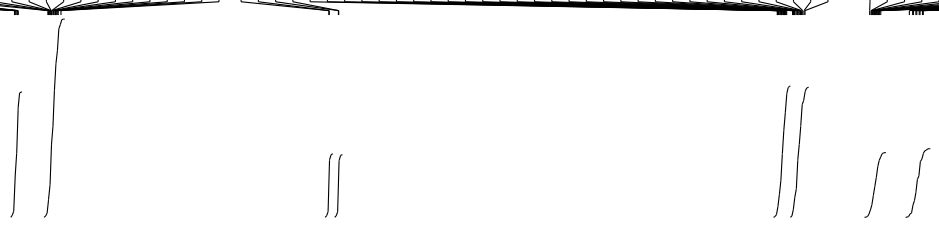<smiles>C=C(c1ccccc1)C1(O[Na])CCC1</smiles>

$1 \mathrm{H} \mathrm{NMR}$

$400 \mathrm{MHz}$

$\mathrm{CDCl}_{3}$

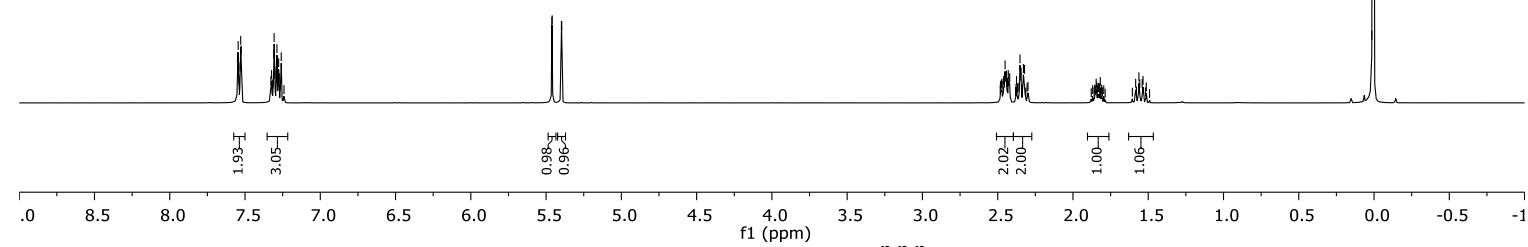

|

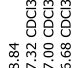

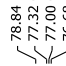

$\stackrel{\substack{\stackrel{m}{m} \\ 1}}{1}$

$\stackrel{\vec{n}}{\stackrel{\vec{n}}{1}}$

$400 \mathrm{MHz}^{2}$
$\mathrm{CDCl}_{3}$

남
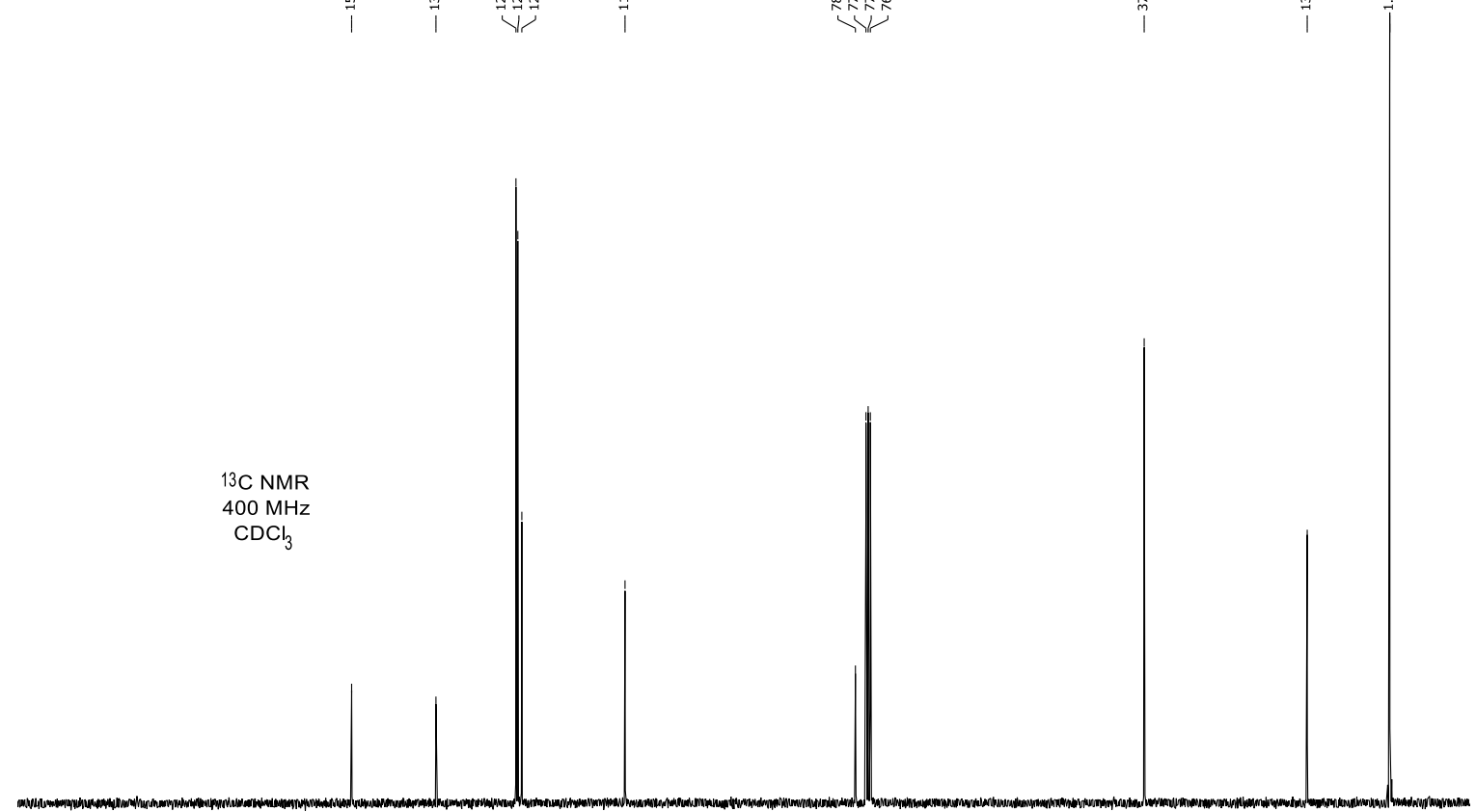

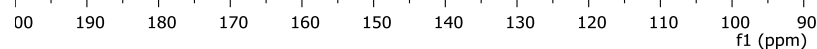
$80 \quad 70 \quad 60 \quad 50$ 


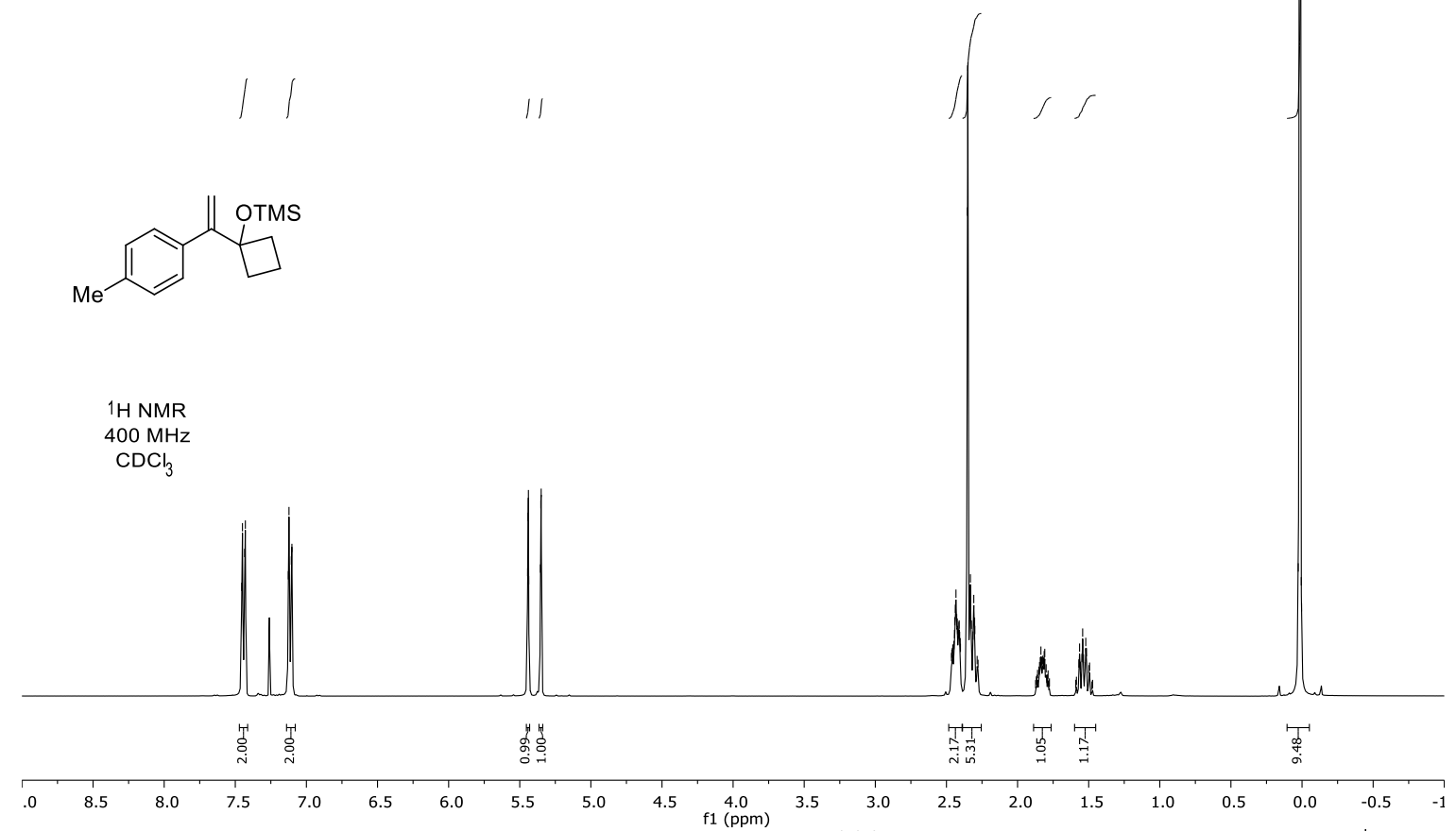

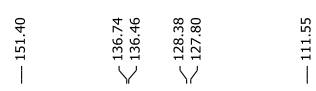

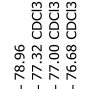

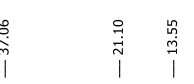

$400 \mathrm{MHz}$

$\mathrm{CDCl}_{3}$
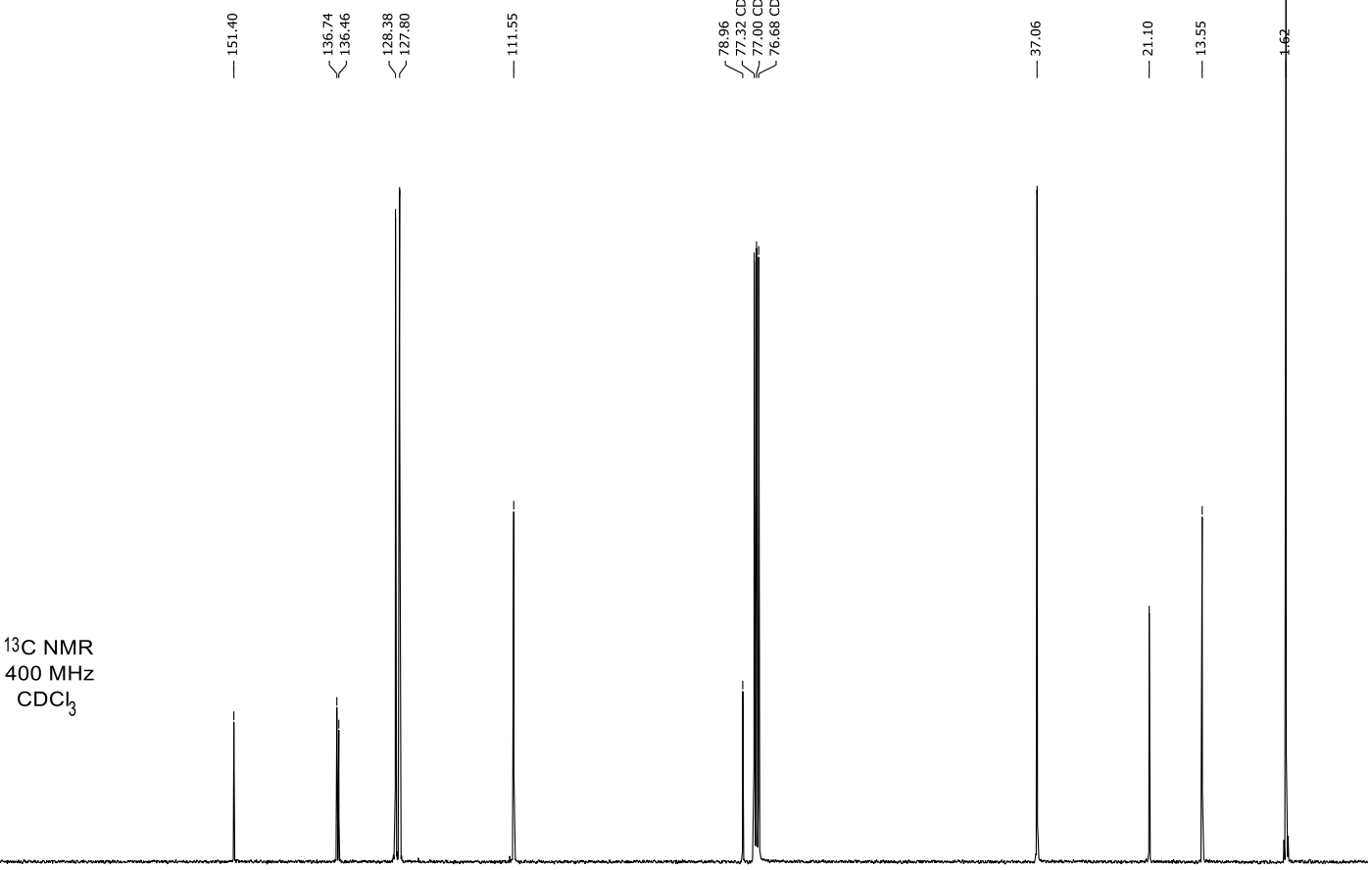

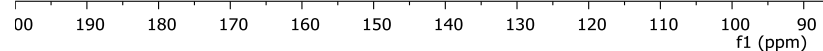
80 


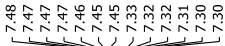

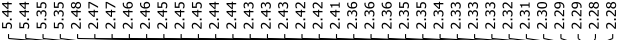
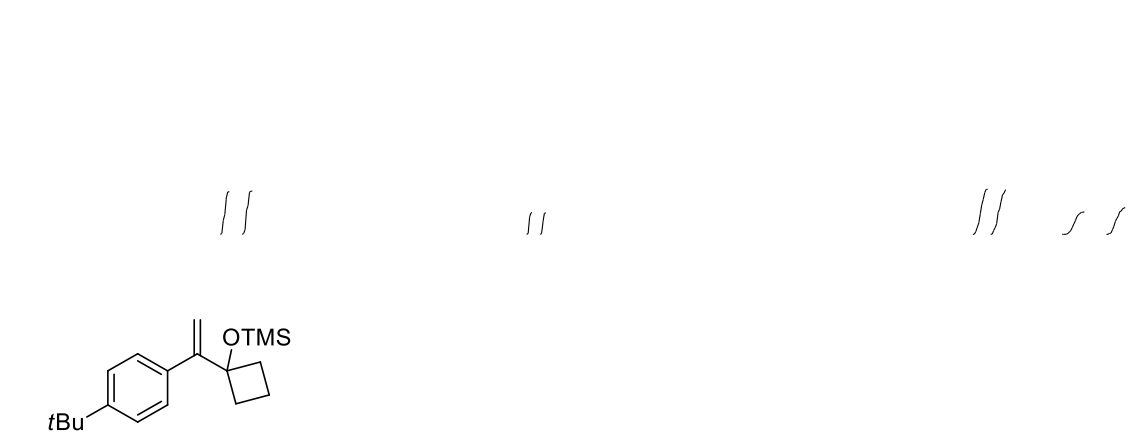

$1 \mathrm{H}$ NMR

$400 \mathrm{MHz}$

$\mathrm{CDCl}_{3}$
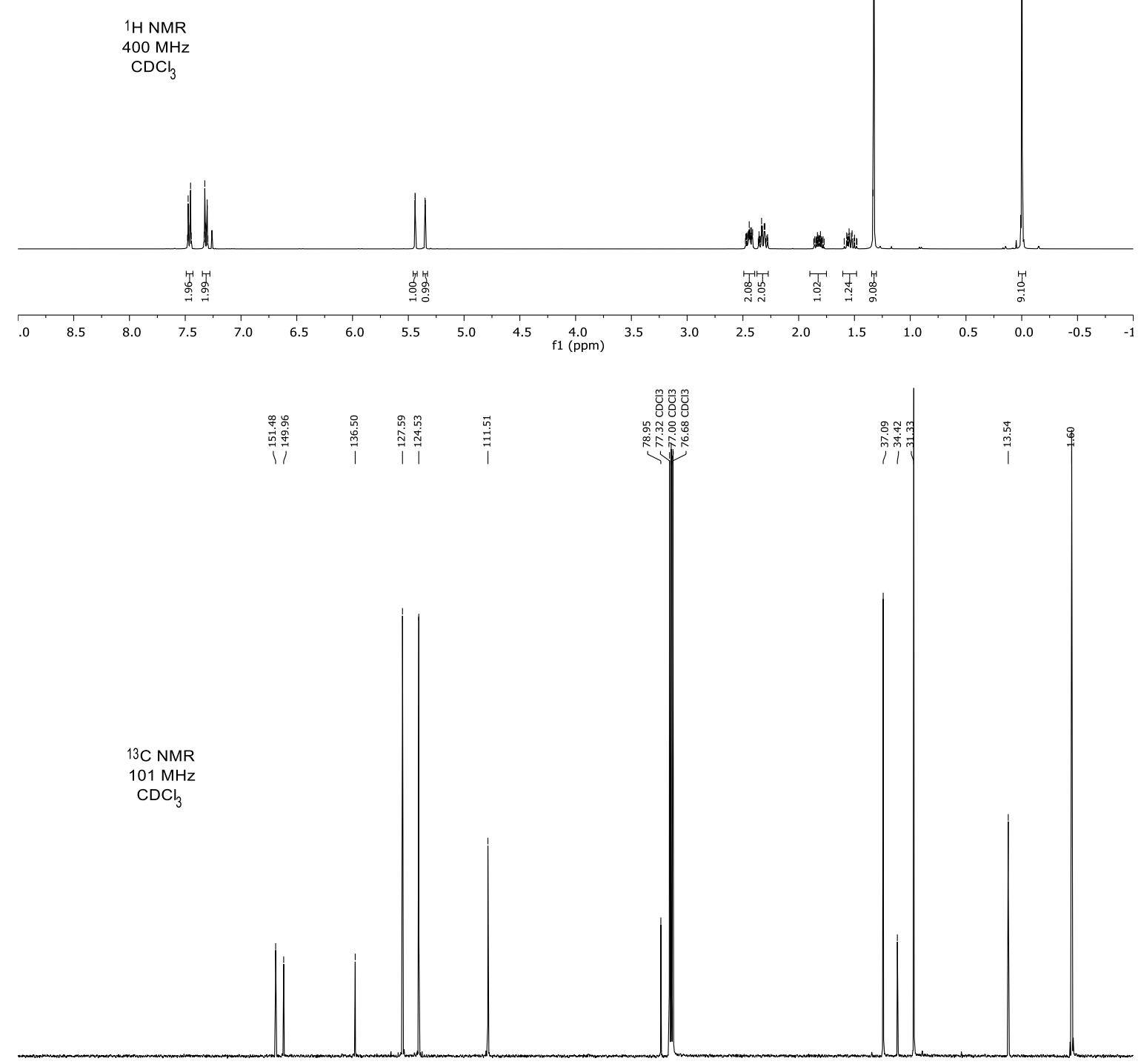

\begin{tabular}{lllllllllll}
\hline 00 & 190 & 180 & 170 & 160 & 150 & 140 & 130 & 120 & 110 & 100 \\
$\mathrm{f} 1(\mathrm{ppm})$
\end{tabular} 


\section{(1-(1-([1,1'-Biphenyl]-4-yl)vinyl)cyclobutoxy)trimethylsilane (4d)}

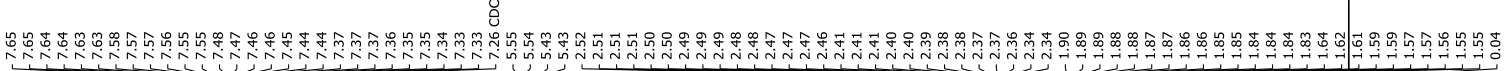

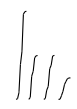

$\iint$
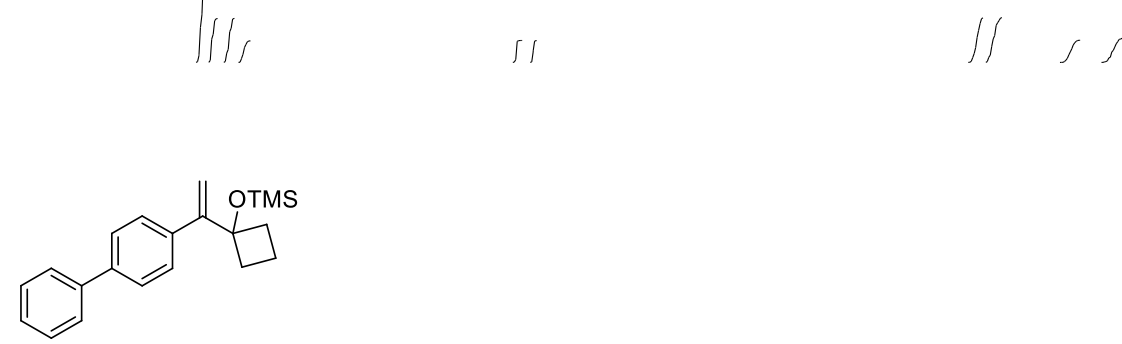

$1 \mathrm{H}$ NMR

$400 \mathrm{MHz}$

$\mathrm{CDCl}_{3}$

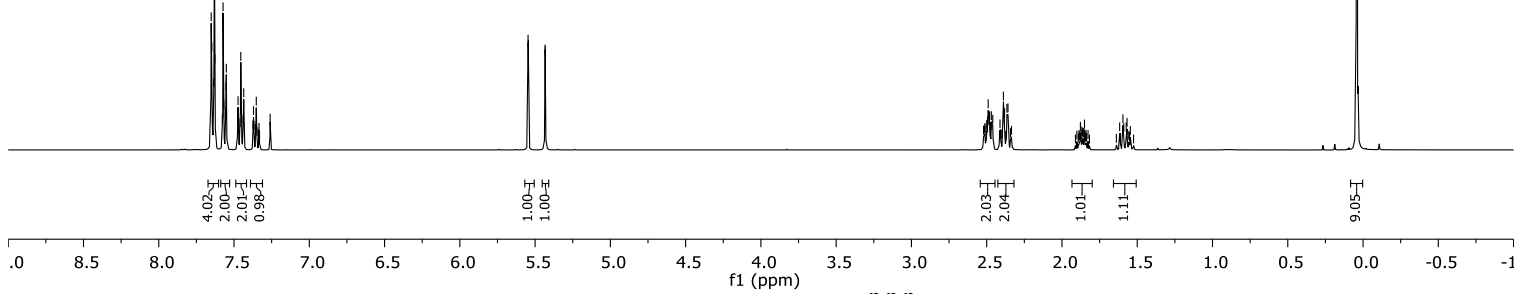

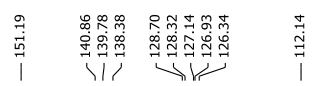

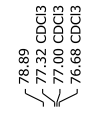

$3 \mathrm{C} \mathrm{NMR}$

$101 \mathrm{MHz}$

$\mathrm{CDCl}_{3}$

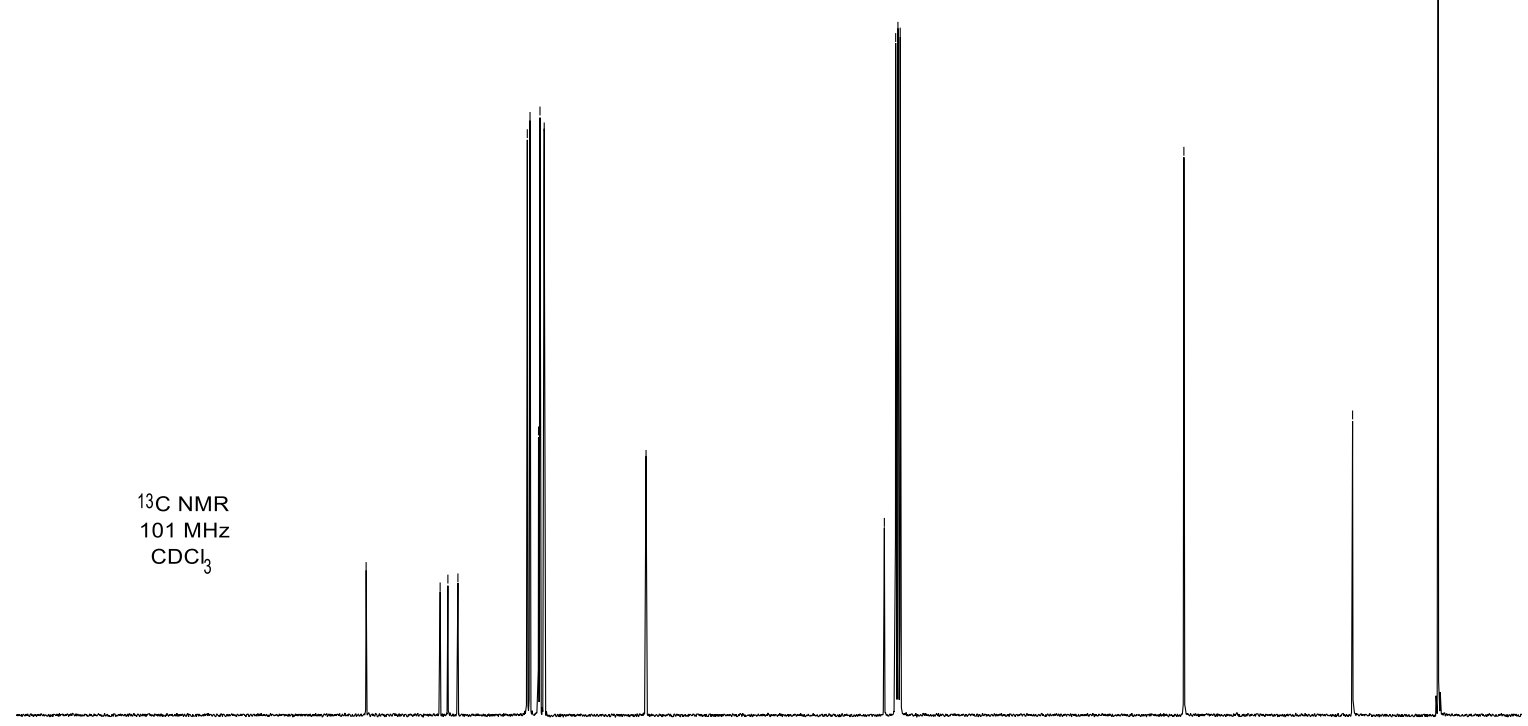

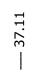

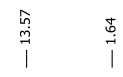

\begin{tabular}{lllllllllll}
\hline 0 & 190 & 180 & 170 & 160 & 150 & 140 & 130 & 120 & 110 & $\begin{array}{c}100 \\
\mathrm{f} 1(\mathrm{ppm})\end{array}$
\end{tabular} 
(1-(1-(4-methoxyphenyl)vinyl)cyclobutoxy)trimethylsilane (4e)

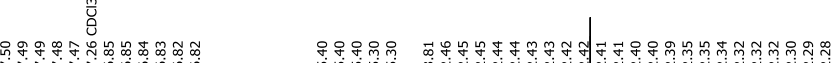

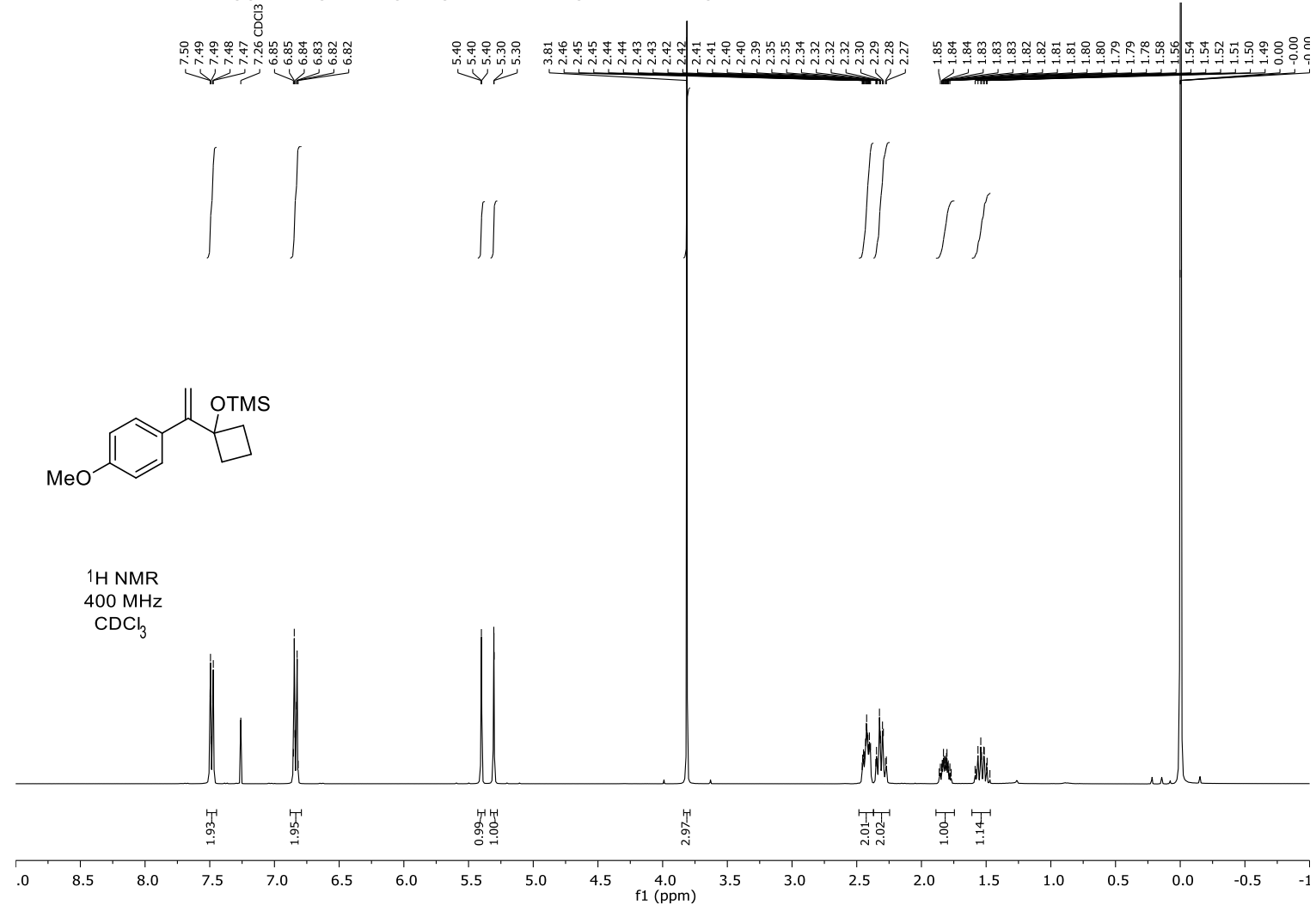

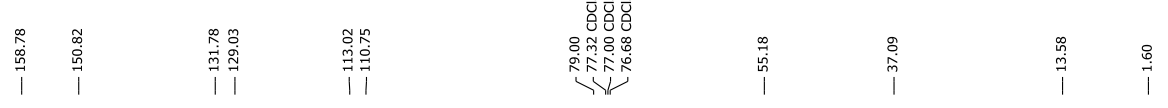

$101 \mathrm{MHz}$

$\mathrm{CDCl}_{3}$

|

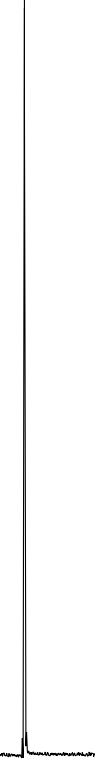

$\begin{array}{lllllllllll}00 & 190 & 180 & 170 & 160 & 150 & 140 & 130 & 120 & 110 & 100 \\ \mathrm{f} 1(\mathrm{ppm})\end{array}$

80 

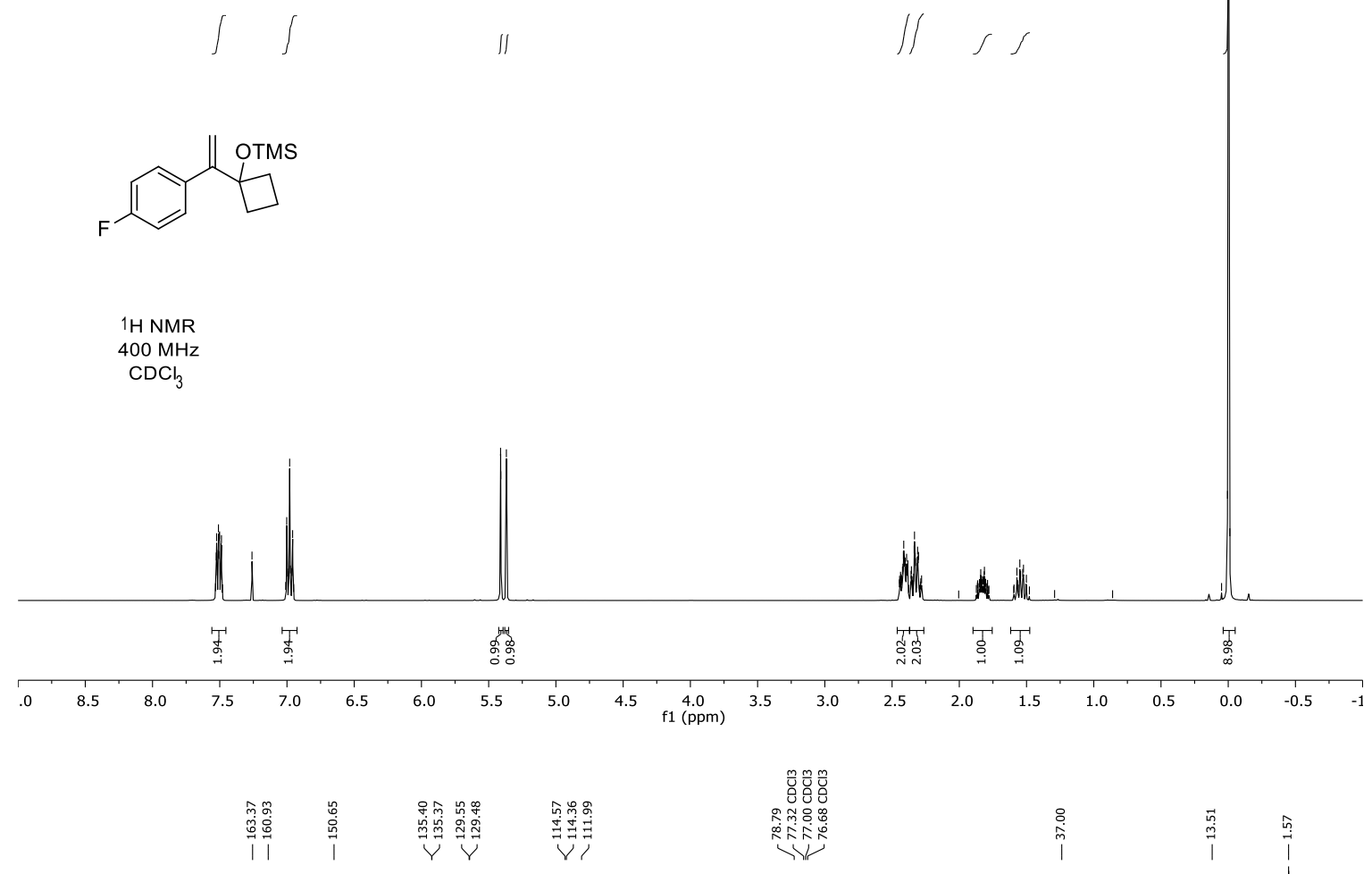

$400 \mathrm{MHz}$

$\mathrm{CDCl}_{3}$

$\stackrel{8}{\stackrel{\circ}{m}}$
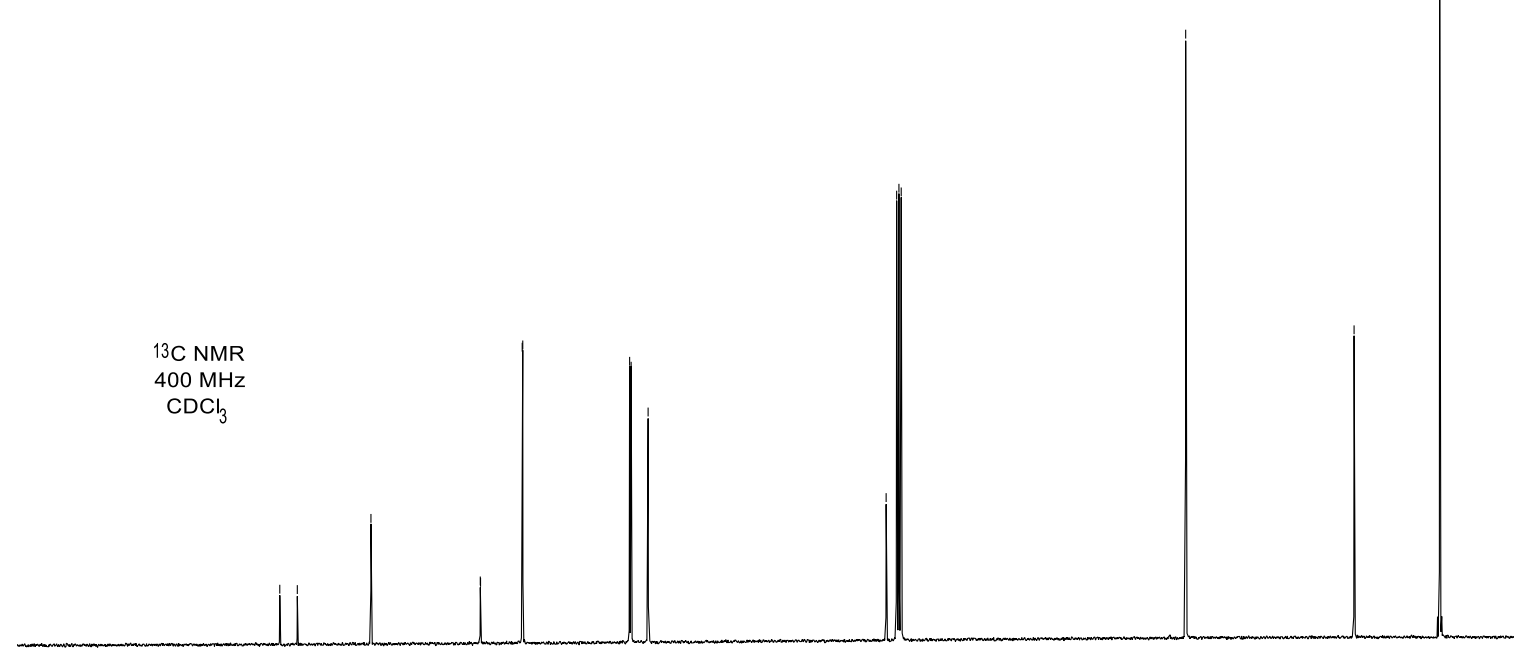

$00 \quad 190$

$\begin{array}{lllllllll}180 & 170 & 160 & 150 & 140 & 130 & 120 & 110 & 100 \begin{array}{c}90 \\ \mathrm{f} 1(\mathrm{ppm})\end{array}\end{array}$ 80 


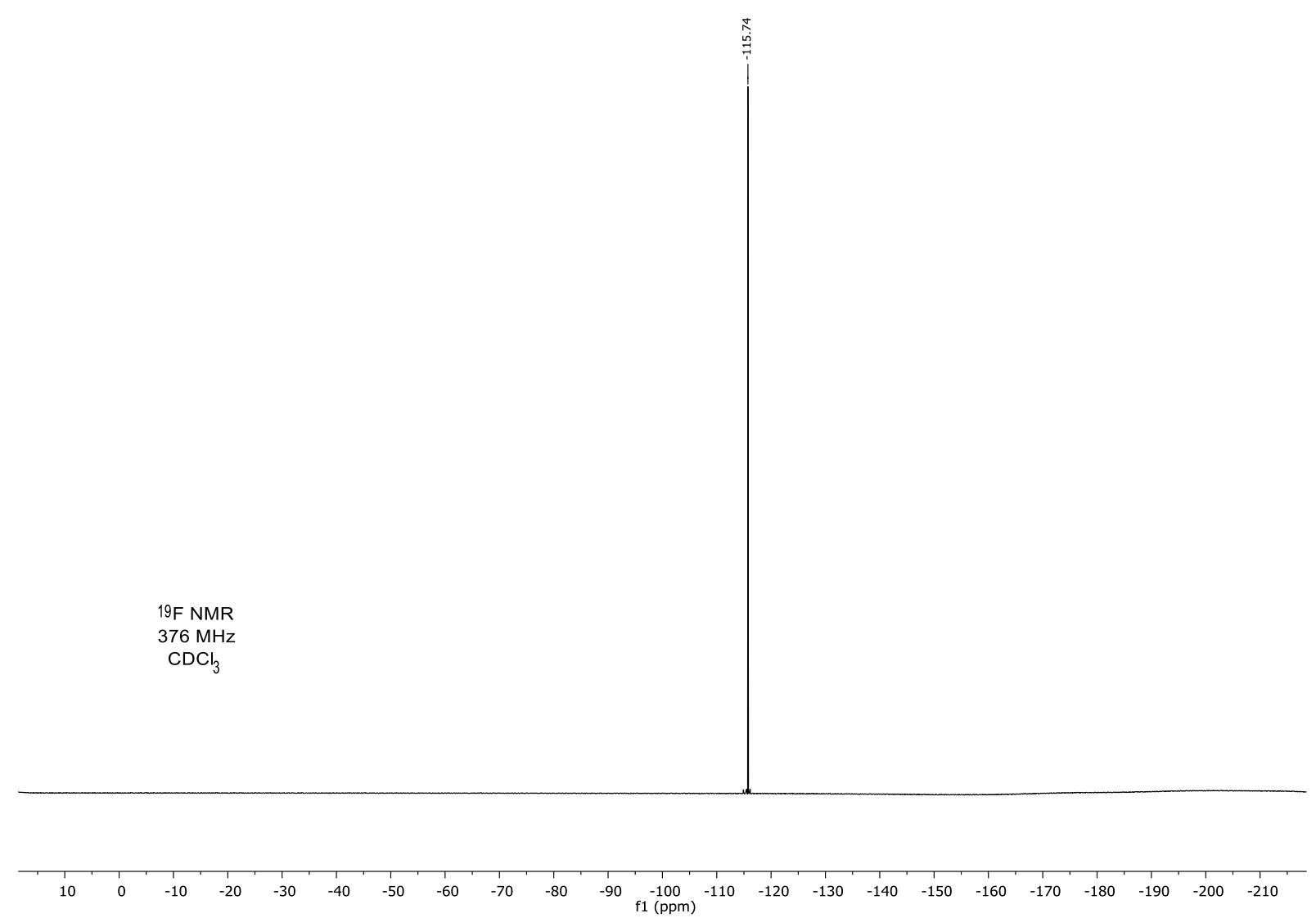




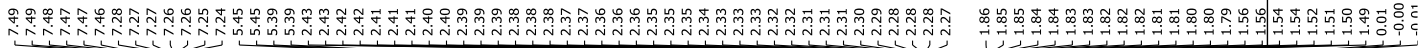
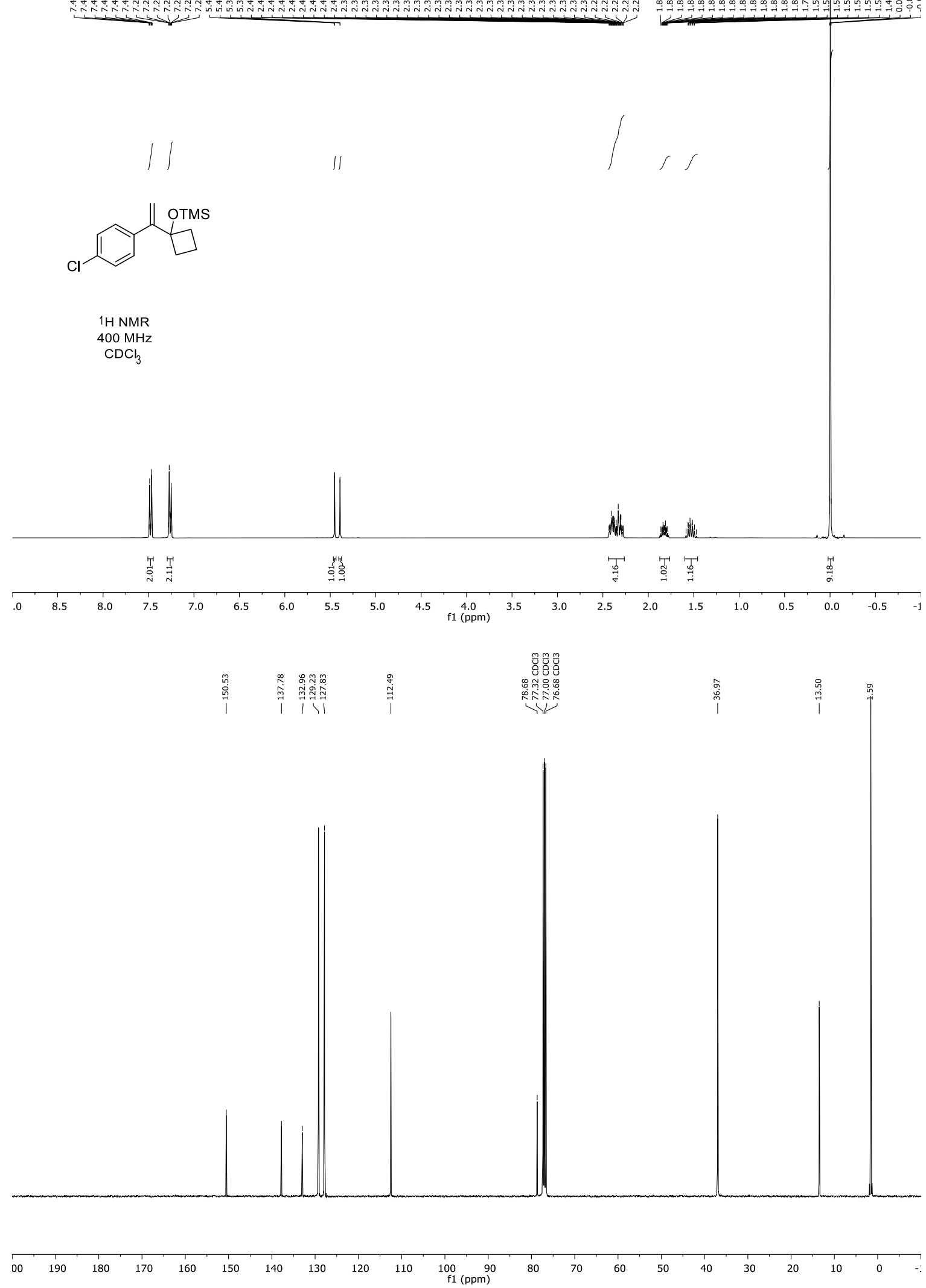
Trimethyl(1-(1-(m-tolyl)vinyl)cyclobutoxy)silane (4h)

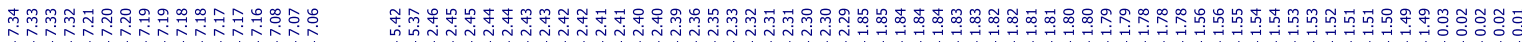
$\iiint$<smiles>C=C(C)C1(c2cccc(C)c2)CCC1</smiles>

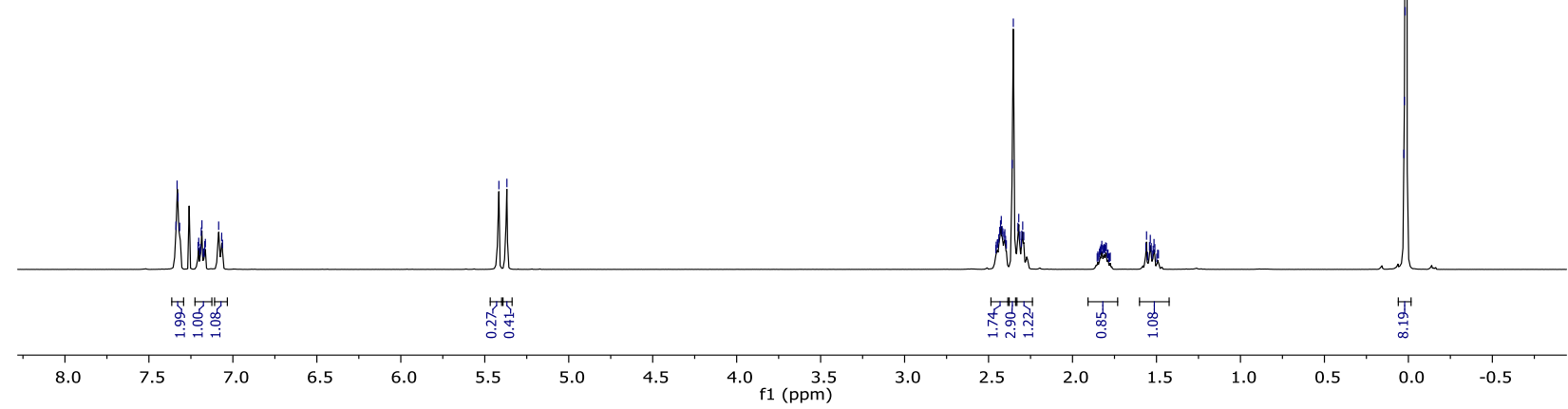

|

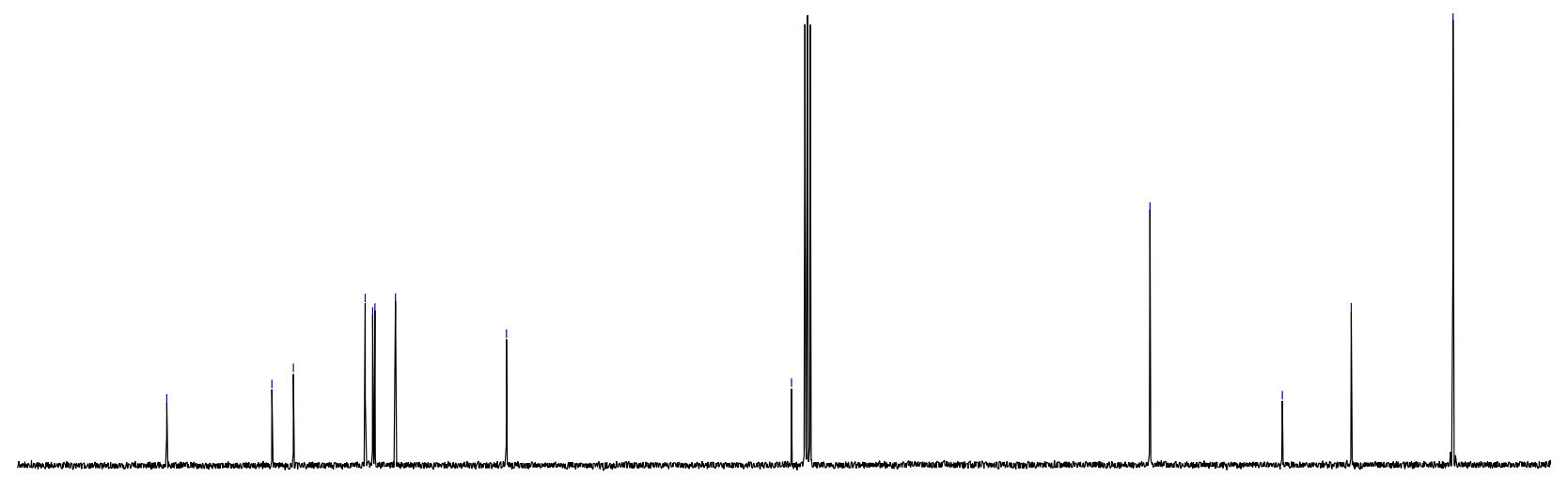




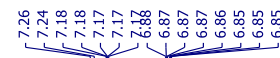

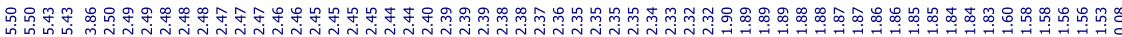

$\iint$

5厂<smiles>C=CCCCCCCC</smiles><smiles>C=C(C)C1(CCC)CC1</smiles>

$4 \mathbf{i}$

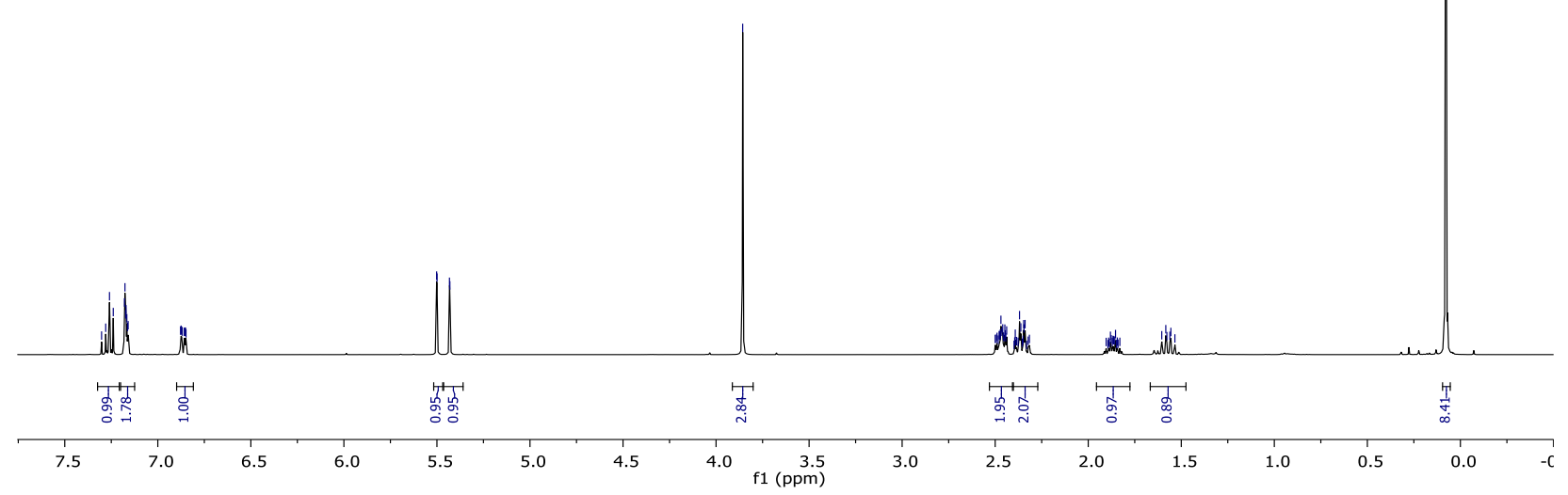

䓪

$\stackrel{\substack{\infty \\ \infty}}{i}$

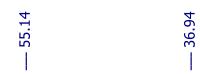

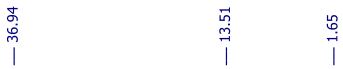

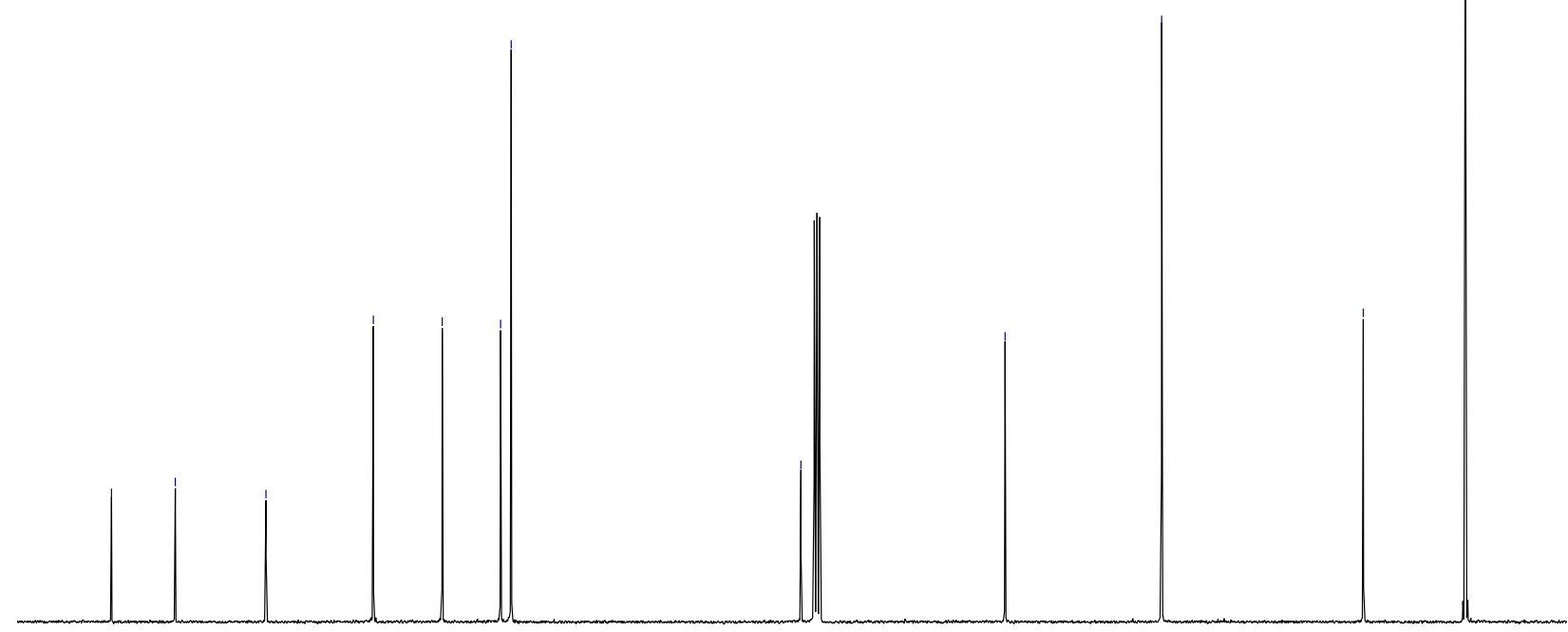
80
$1(\mathrm{ppm})$ 70 


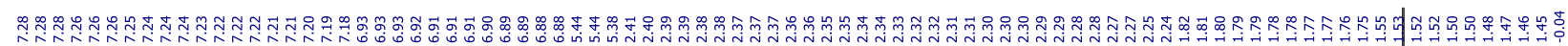

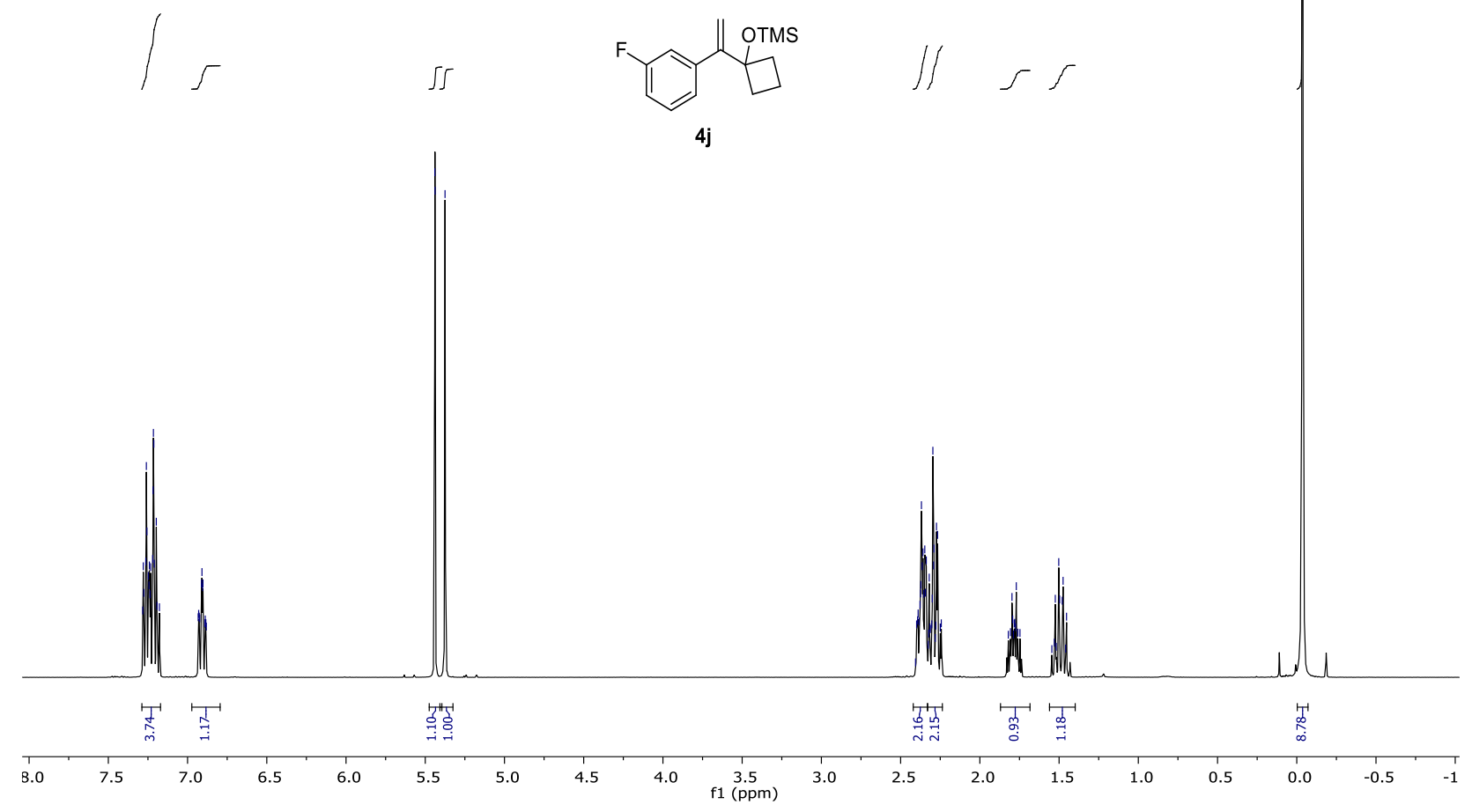

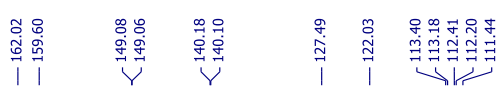

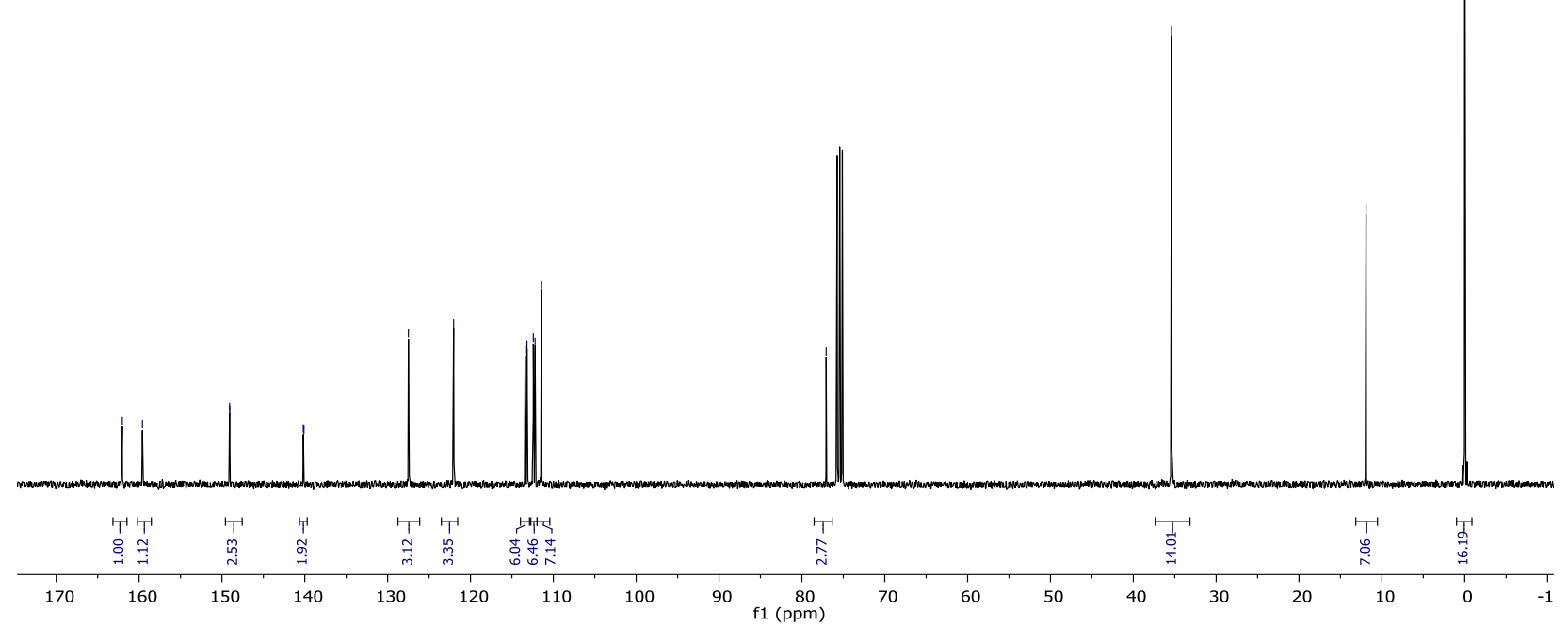


Trimethyl(1-(1-(o-tolyl)vinyl)cyclobutoxy)silane (4k).

产
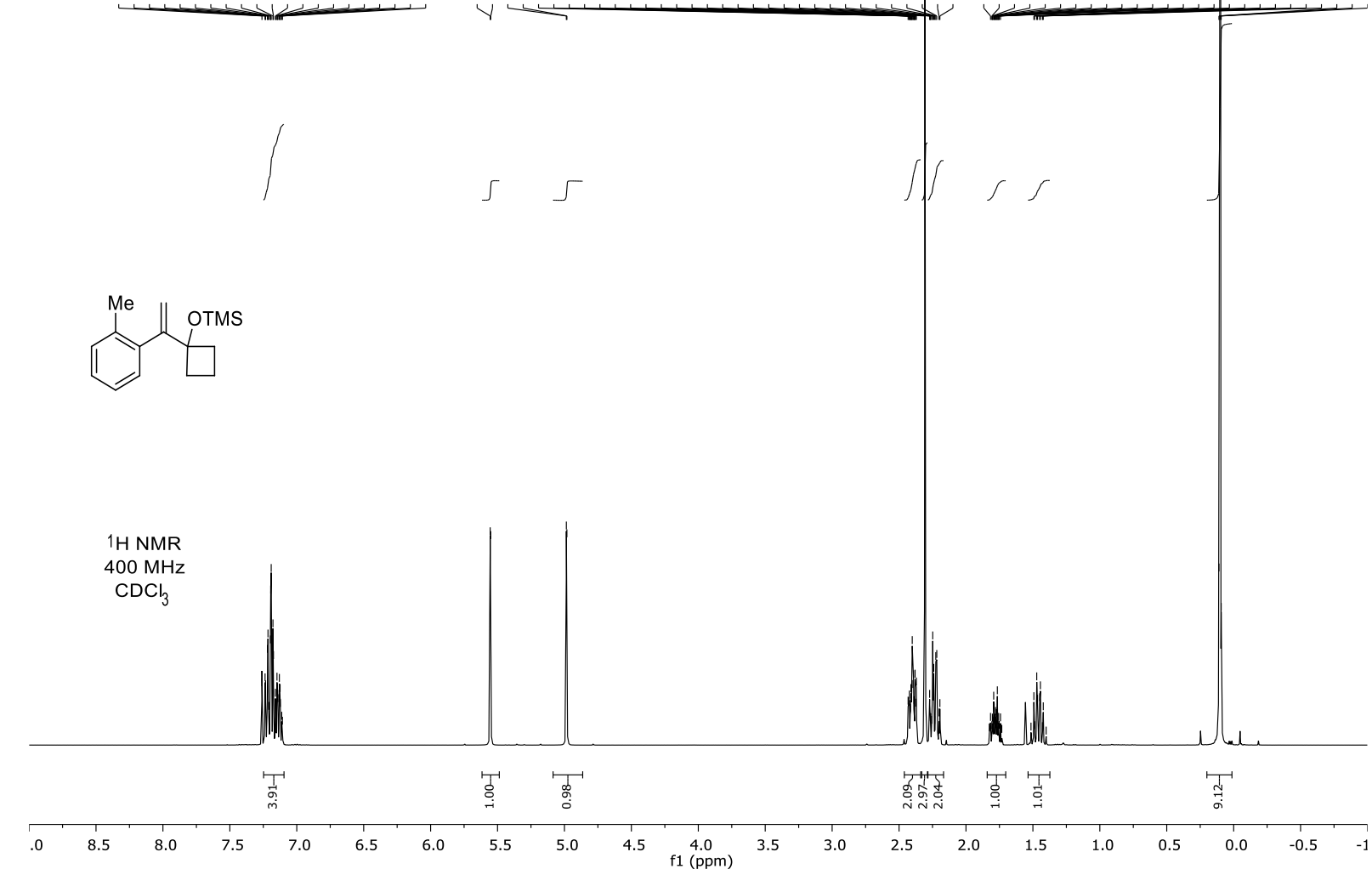

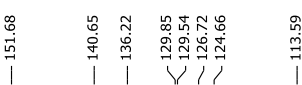

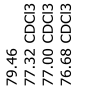

$\mathrm{CDCl}_{3}$

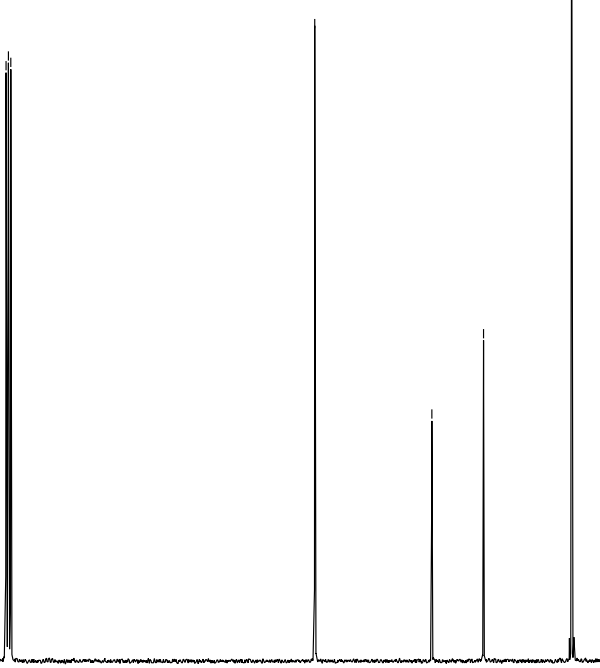

oo

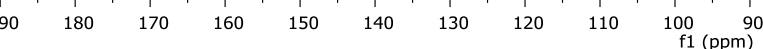


(1-(1-(3,4-Dimethylphenyl)vinyl)cyclobutoxy)trimethylsilane (4I)
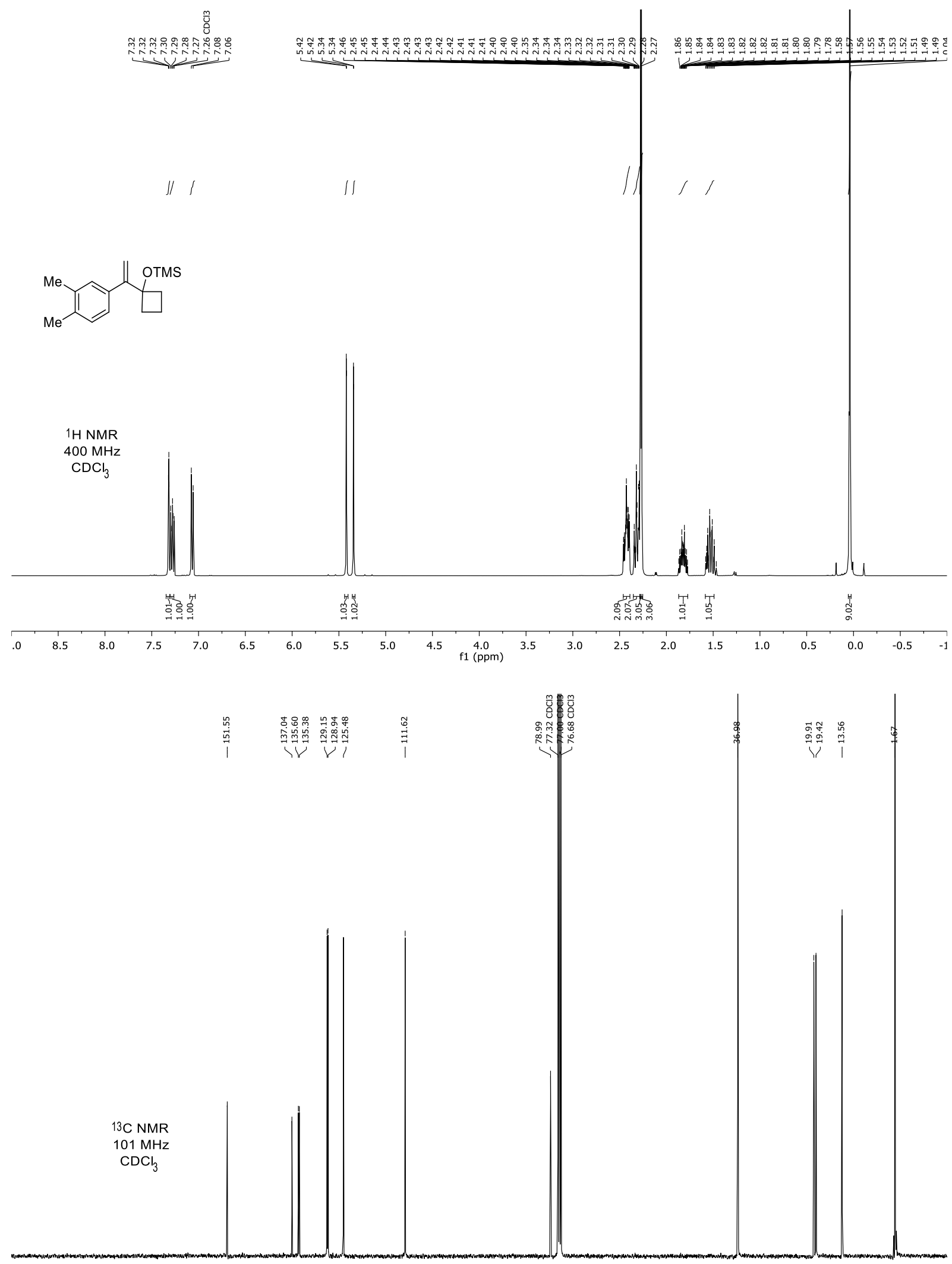

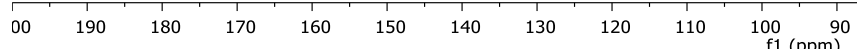




\section{(1-(1-(3,4-Dimethoxyphenyl)vinyl)cyclobutoxy)trimethylsilane (4m)}

范

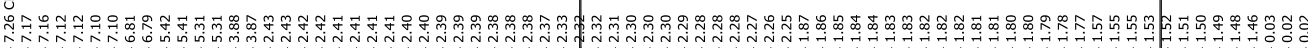<smiles>C=C(c1ccc(OC)c(OC)c1)C1([O+])CCC1</smiles>

$\mathrm{CDCl}_{3}$
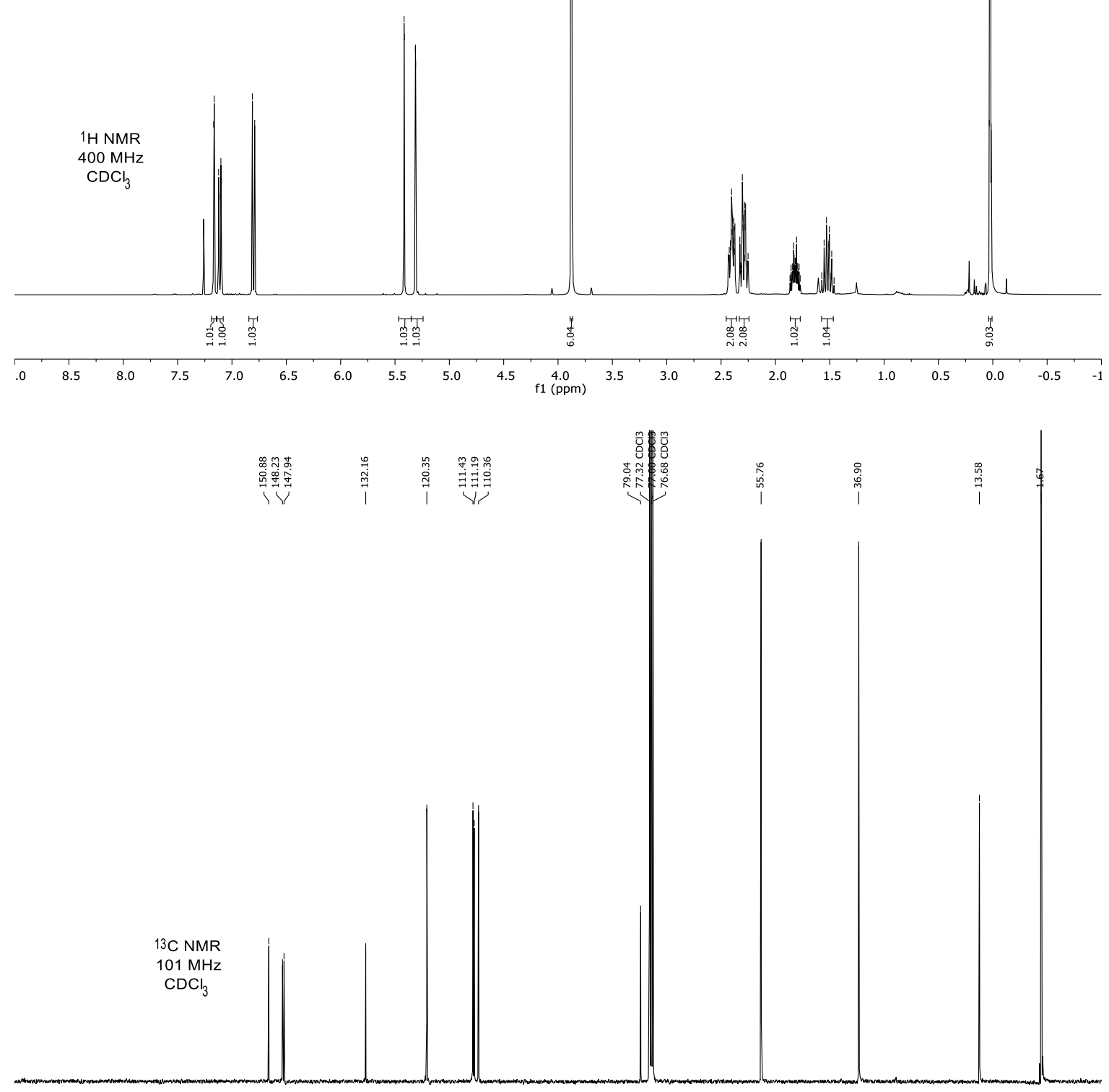

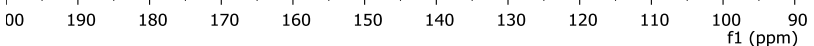


(1-(1-(Benzo[d][1,3]dioxol-5-yl)vinyl)cyclobutoxy)trimethylsilane (4n)

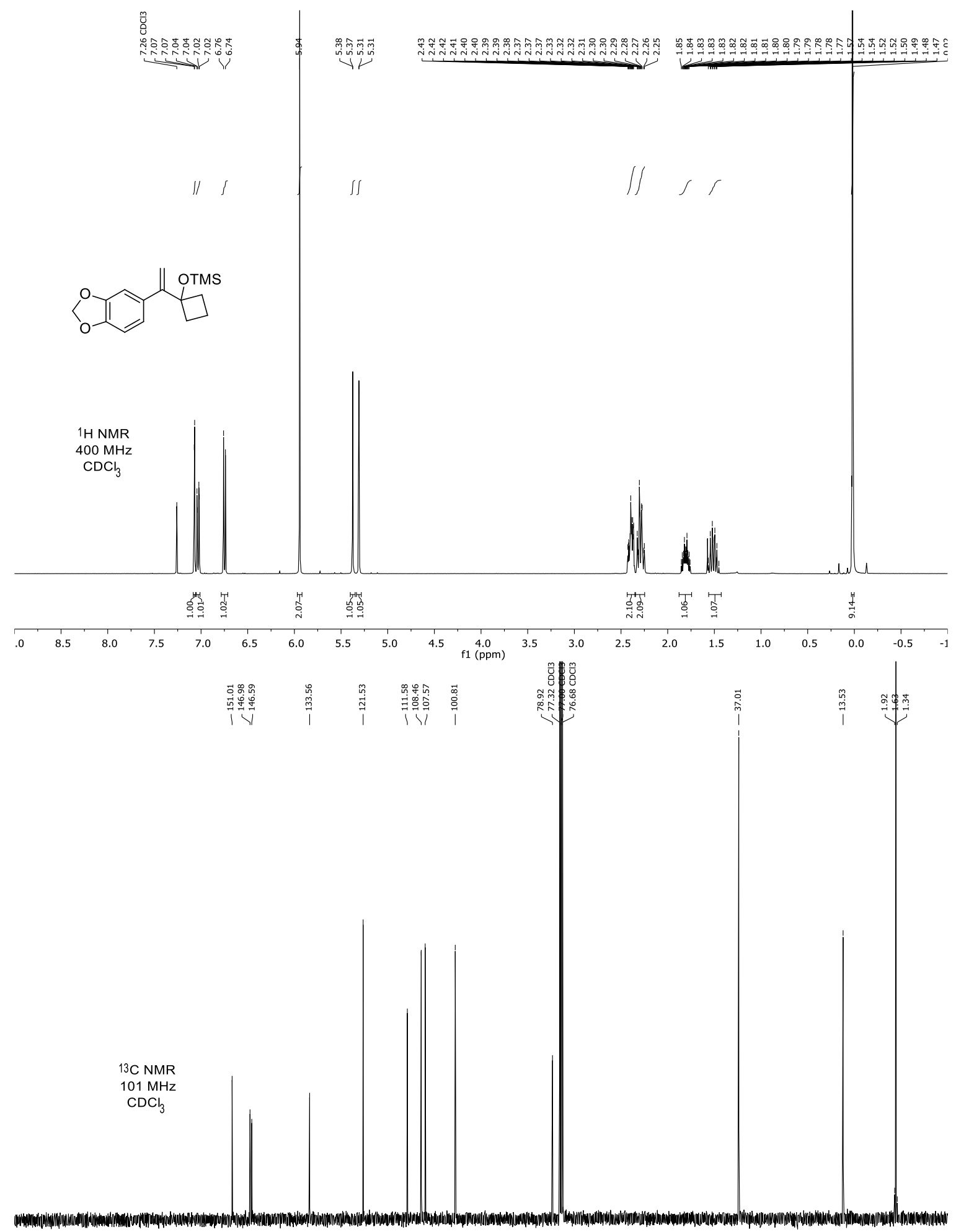

$\begin{array}{lllllllllll}00 & 190 & 180 & 170 & 160 & 150 & 140 & 130 & 120 & 110 & 100 \\ \mathrm{f} 1(\mathrm{ppm}) & 90\end{array}$ 
Trimethyl(1-(1-(naphthalen-2-yl)vinyl)cyclobutoxy)silane (40)
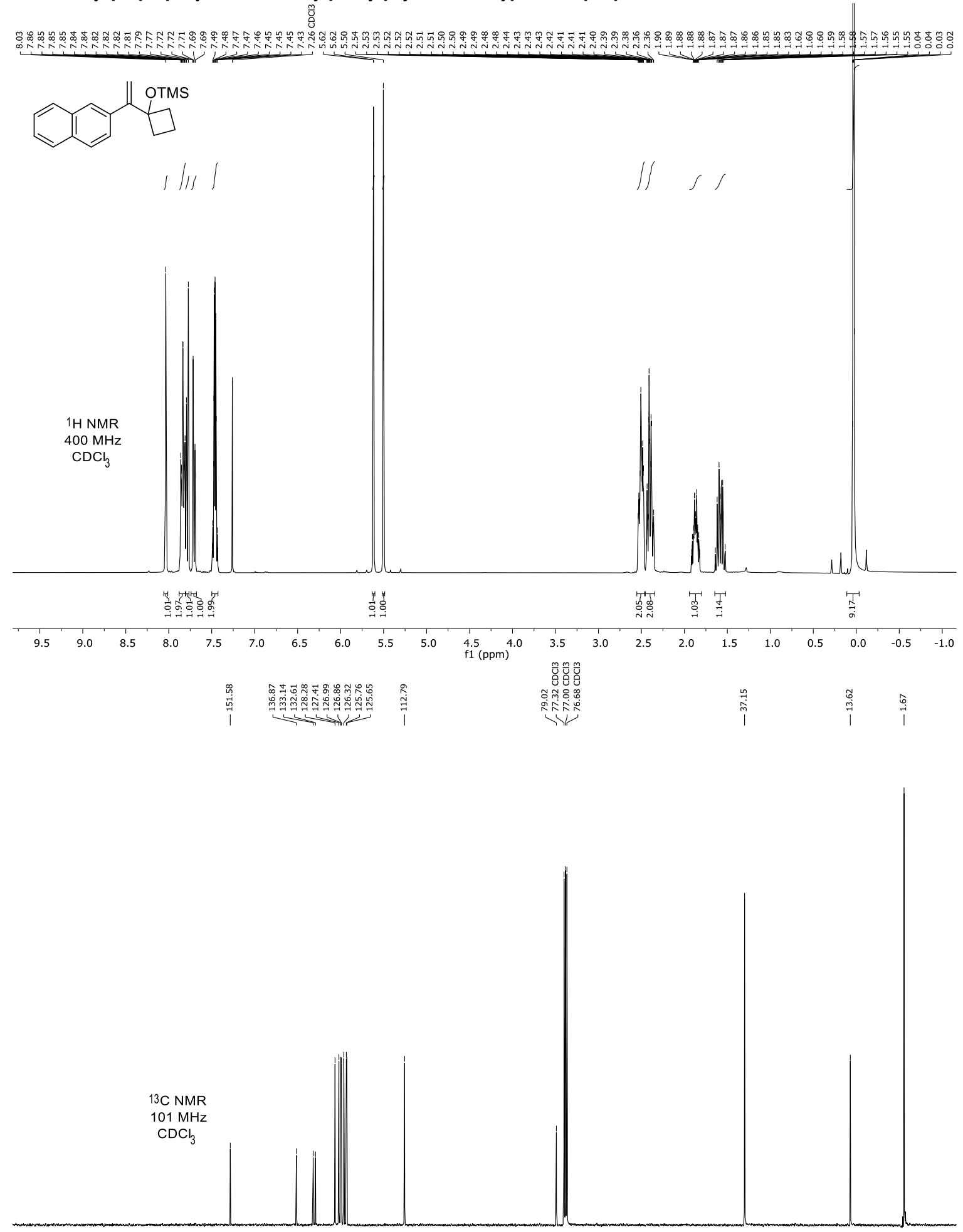

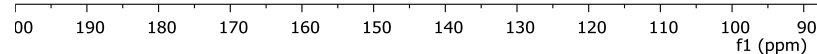
80 
Trimethyl(1-(1-(naphthalen-1-yl)vinyl)cyclobutoxy)silane (4p).
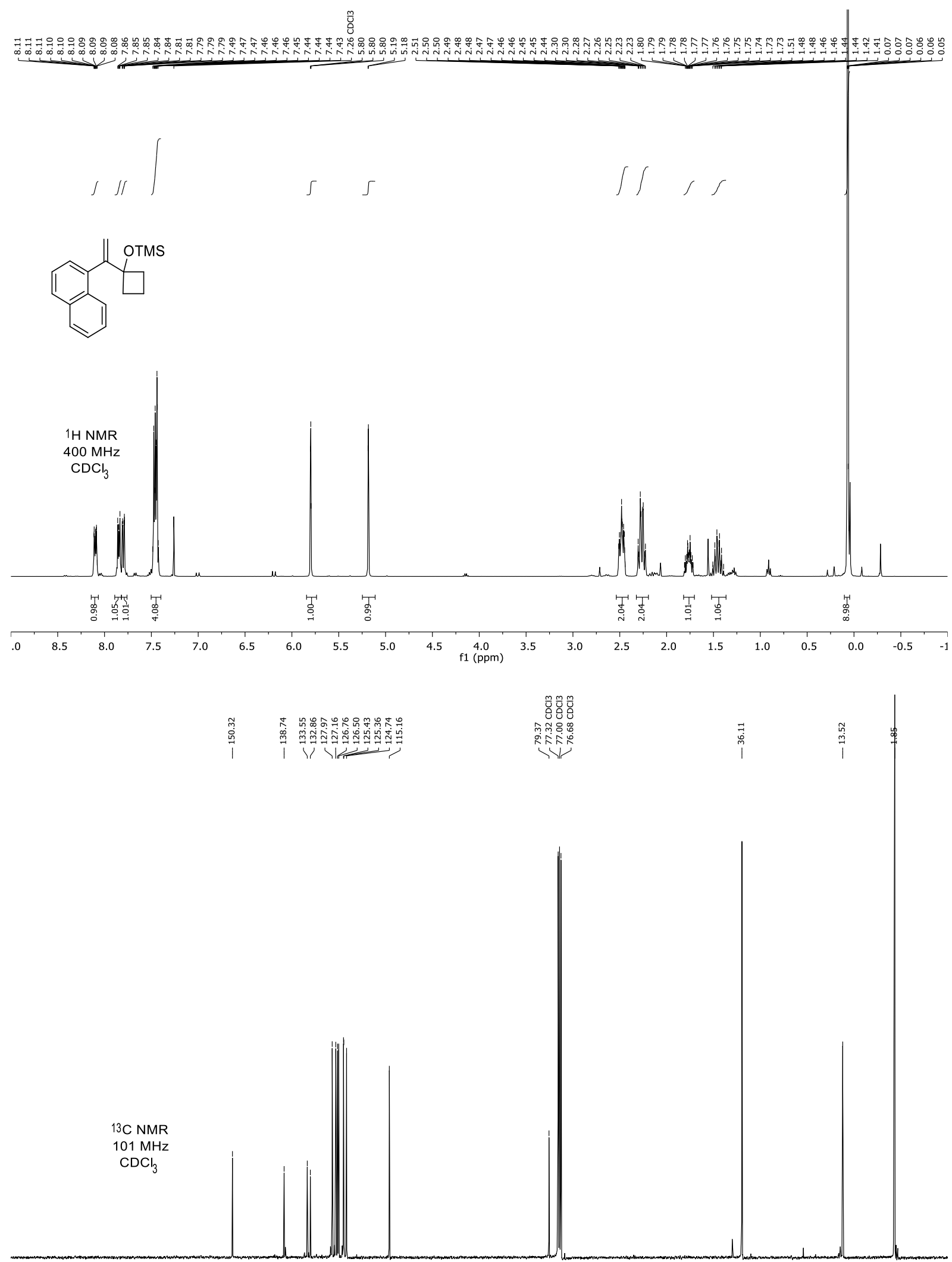

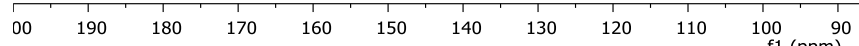


Trimethyl((3-(1-phenylvinyl)oxetan-3-yl)oxy)silane (4q)

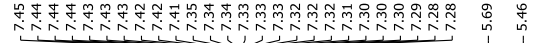
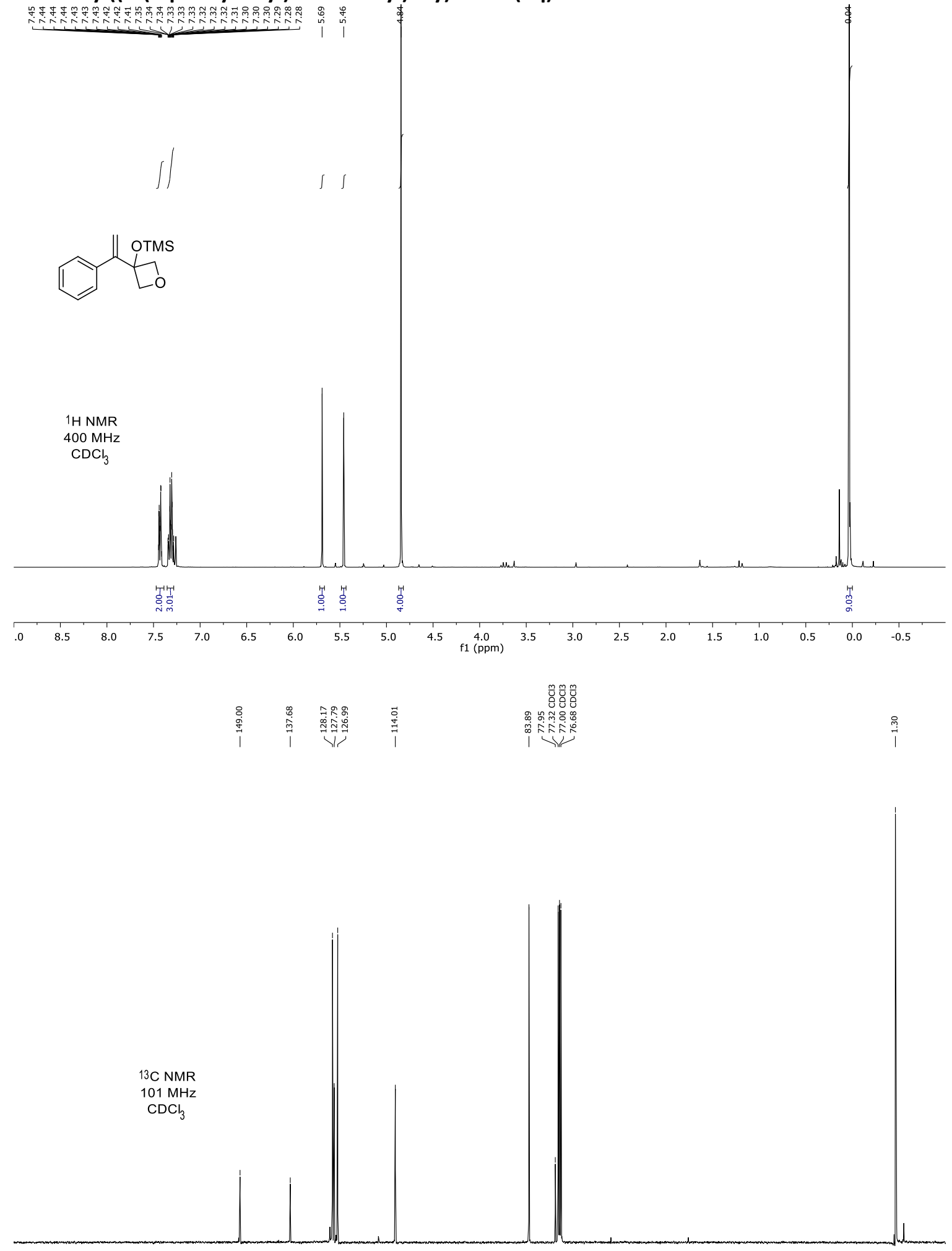

\begin{tabular}{lllllllllll}
\hline 00 & 190 & 180 & 170 & 160 & 150 & 140 & 130 & 120 & 110 & 100 \\
$\mathrm{f} 1(\mathrm{ppm})$
\end{tabular} 
tert-Butyl 3-(1-phenylvinyl)-3-((trimethylsilyl)oxy)azetidine-1-carboxylate (4r)

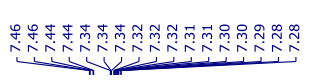

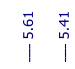

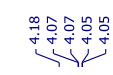

$\int$

$\ulcorner\varsigma$
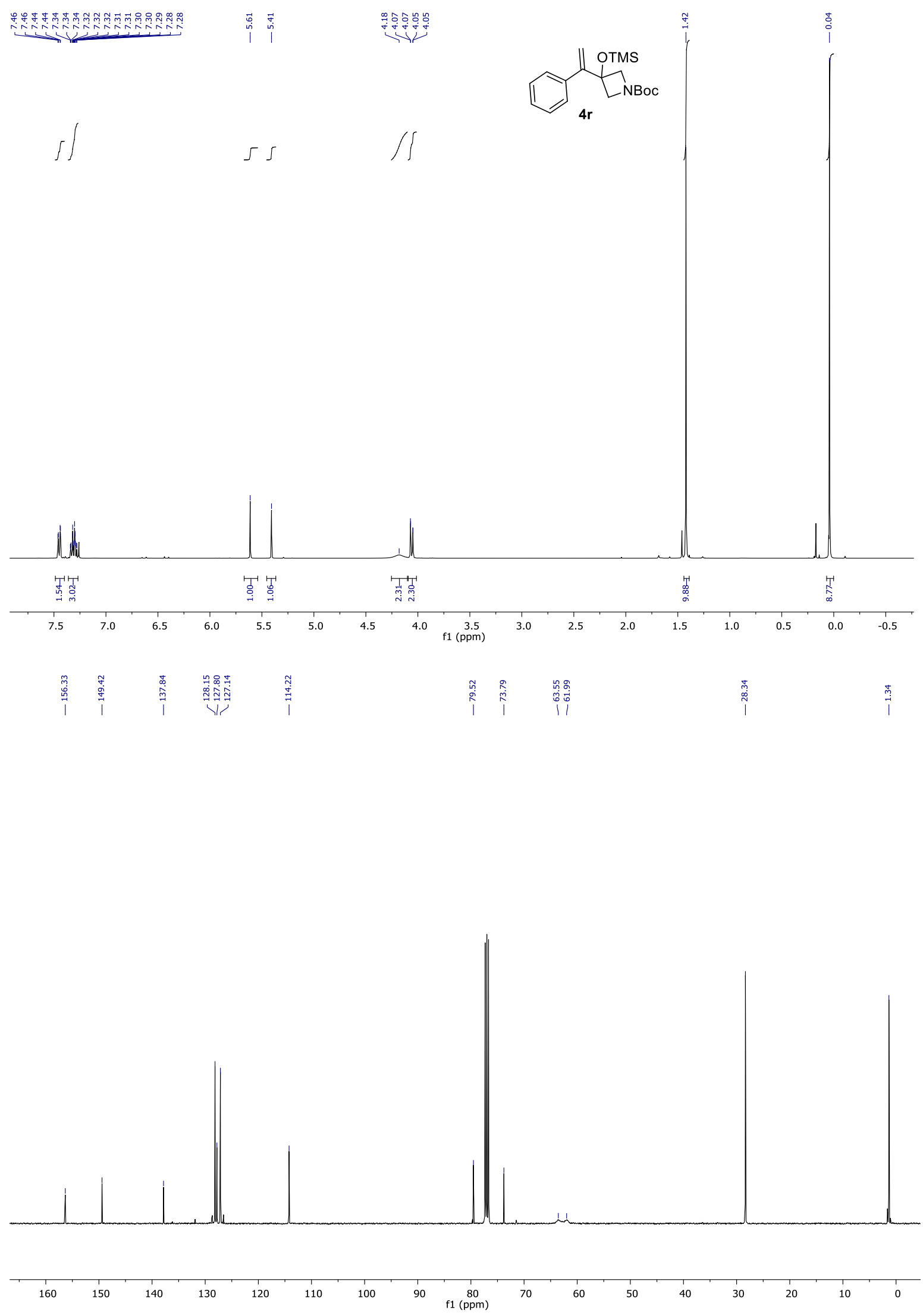

43 


\section{(1-(3,4-Dihydronaphthalen-1-yl)cyclobutoxy)trimethylsilane (4s)}

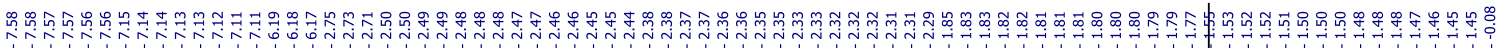

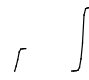

$$
\text { 厂 }
$$
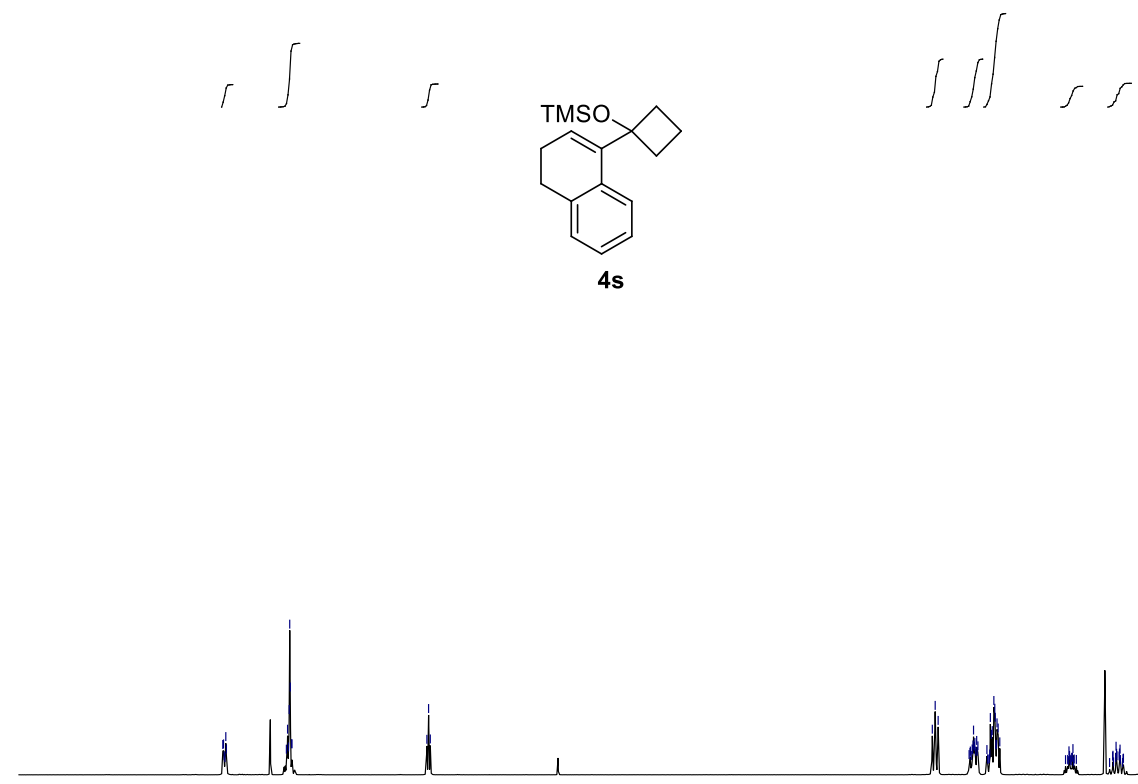

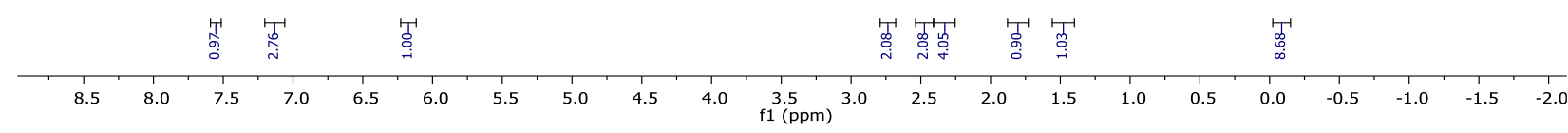

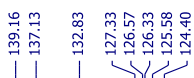

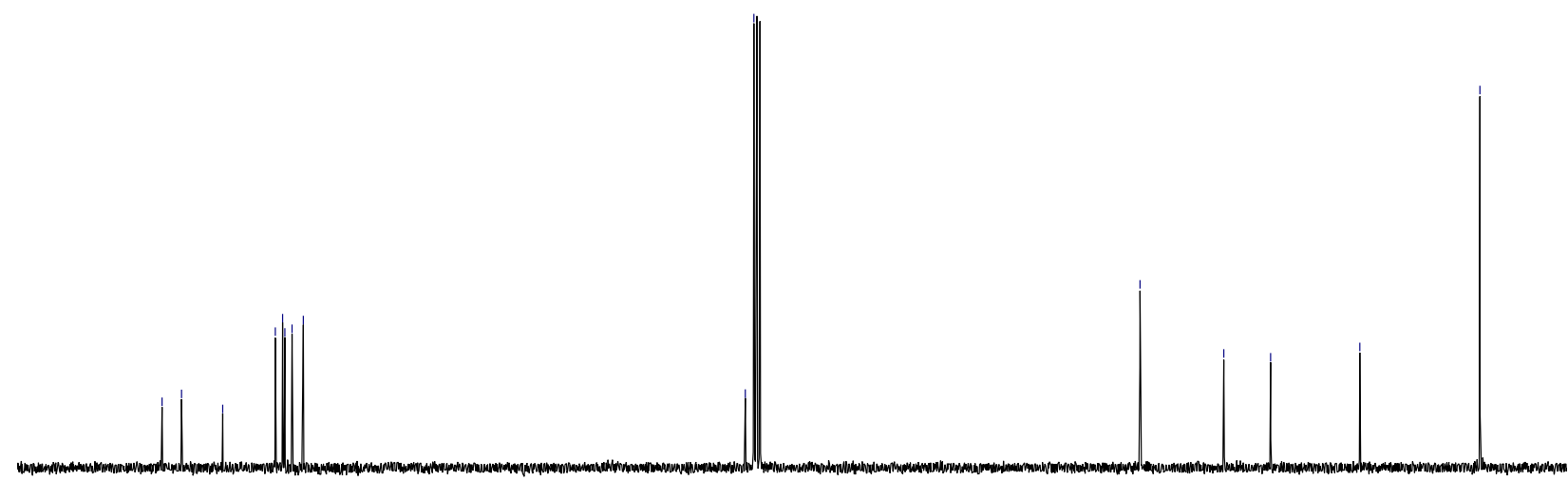

$150-140$

$80 \quad 70$

60

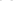

40

30 
Trimethyl(1-(1-(4-(trifluoromethyl)phenyl)vinyl)cyclobutoxy)silane (4t).
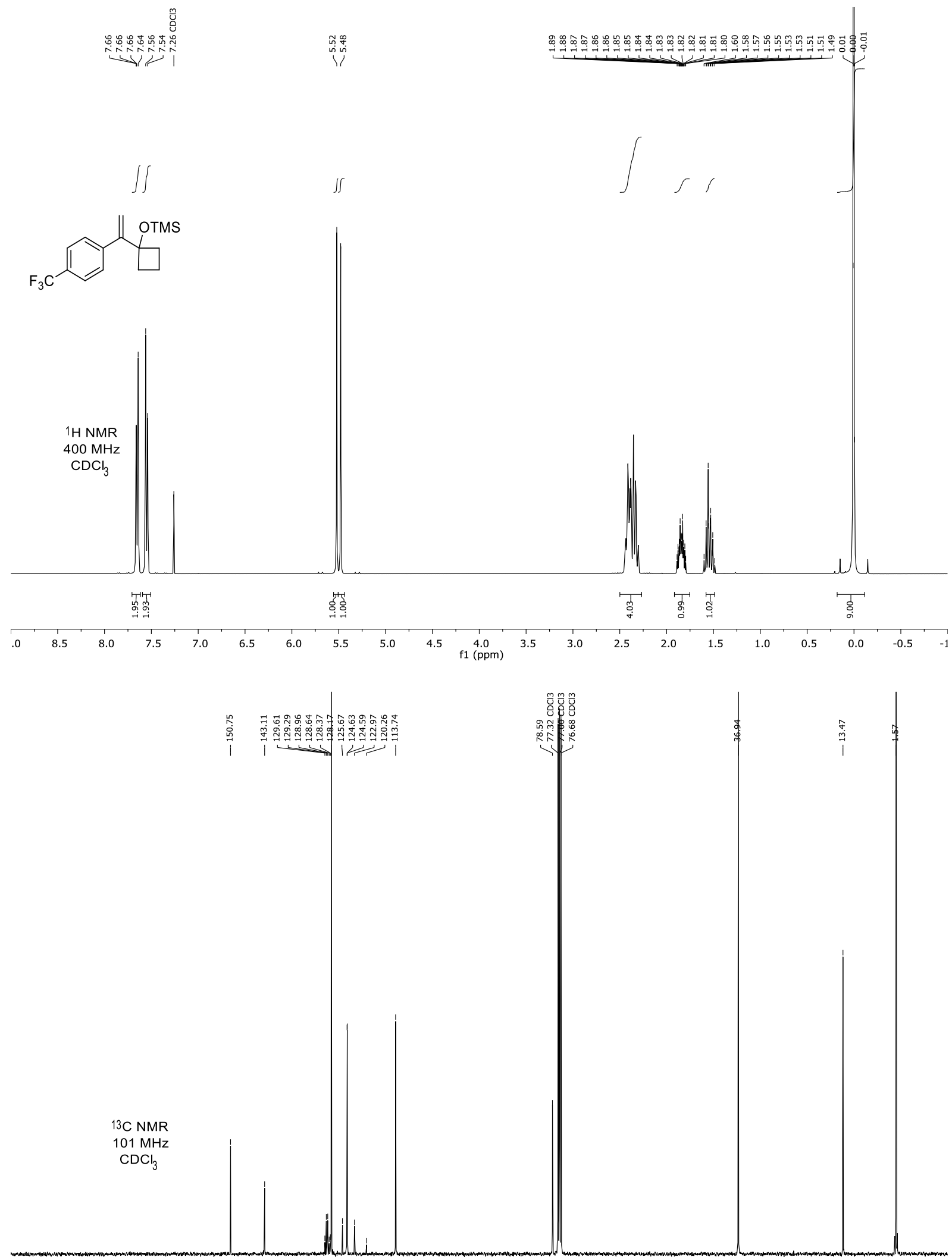

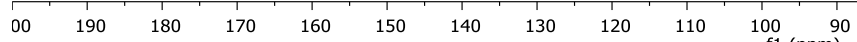




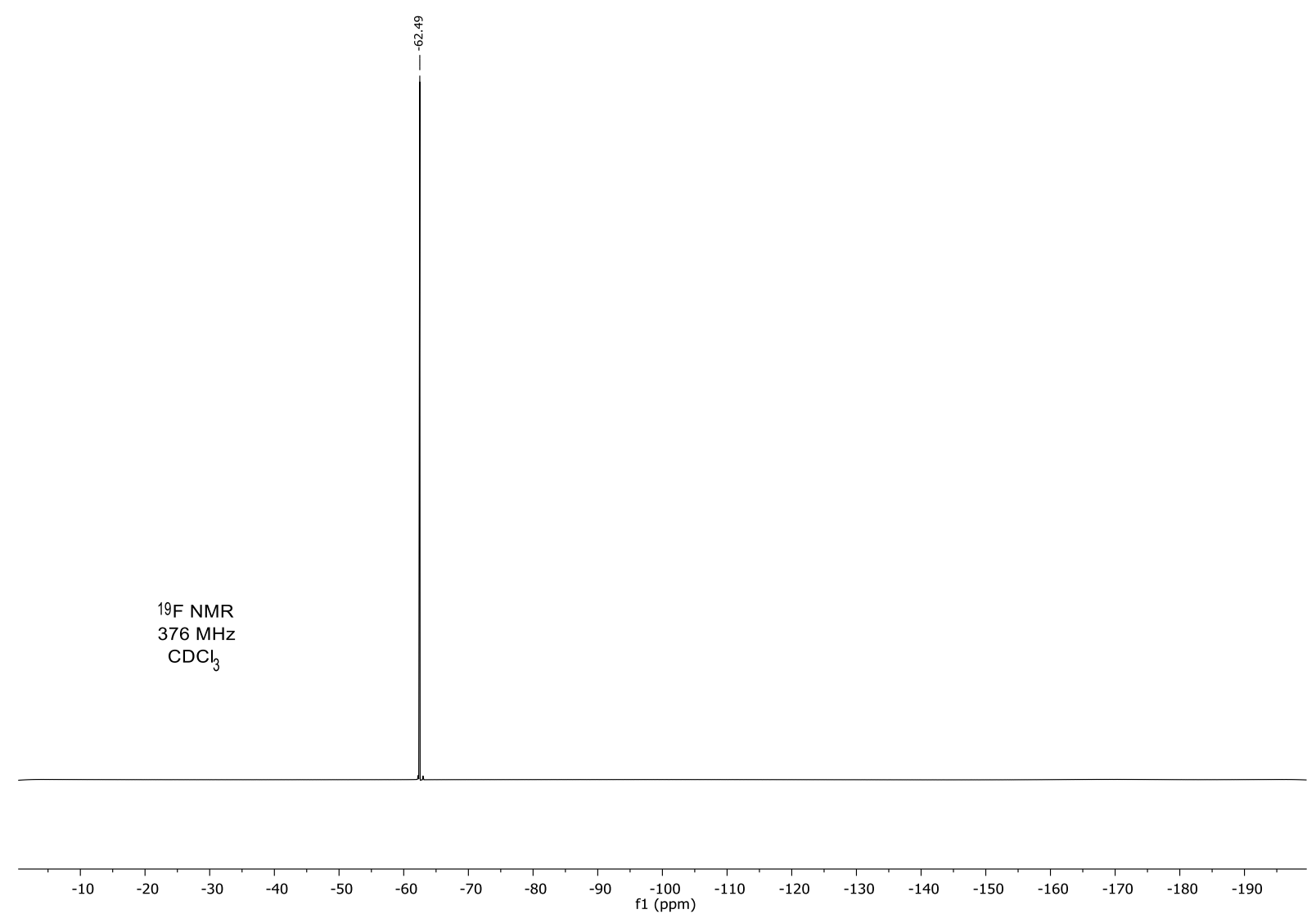


2-(Azidomethyl)-2-phenylcyclopentanone (6a)
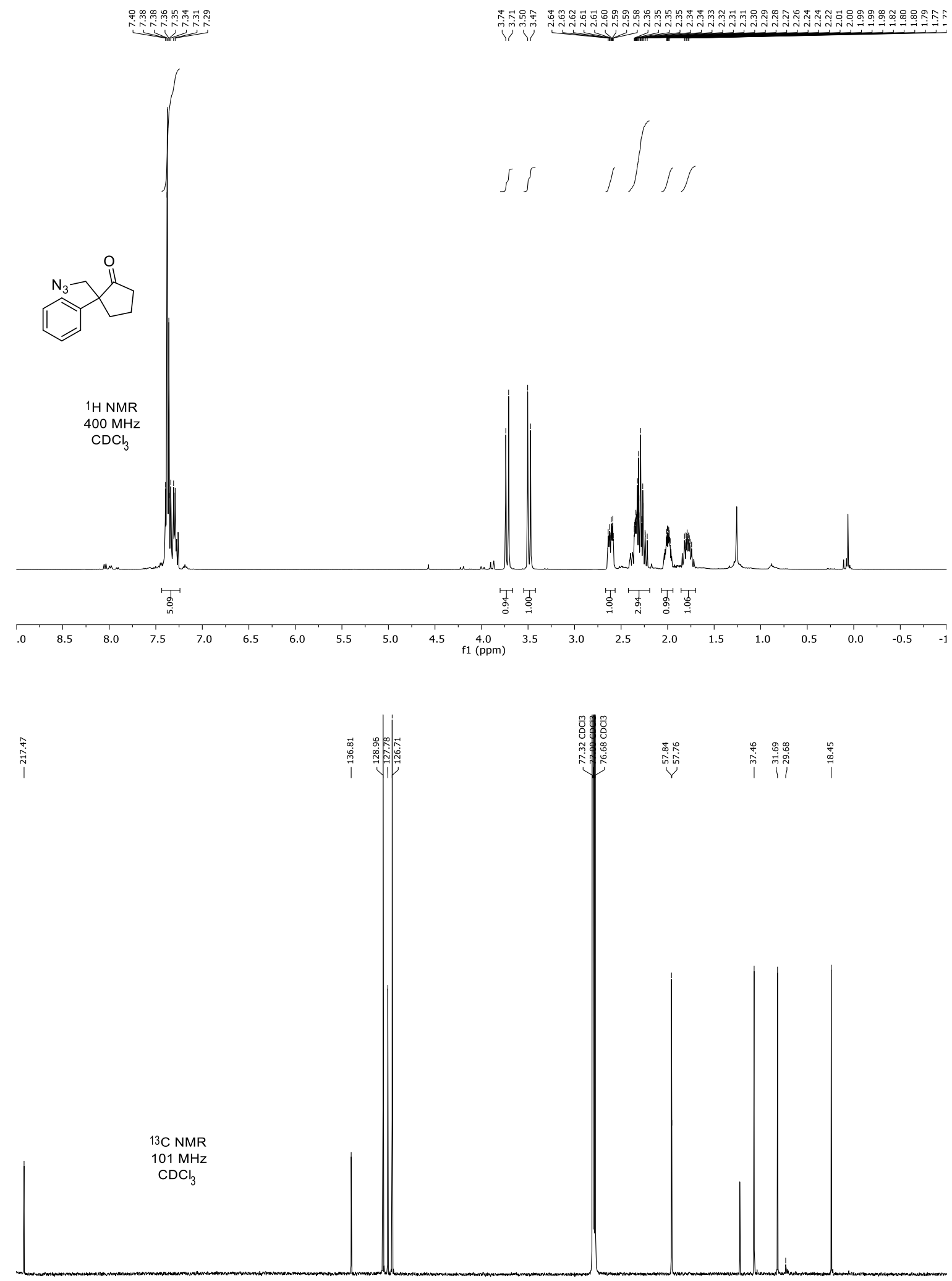

$\begin{array}{llllllllllll}210 & 200 & 190 & 180 & 170 & 160 & 150 & 140 & 130 & 120 & 110 & 100 \\ \mathrm{f} 1(\mathrm{ppm})\end{array}$ 
2-(Azidomethyl)-2-(p-tolyl)cyclopentanone (6b)

峞

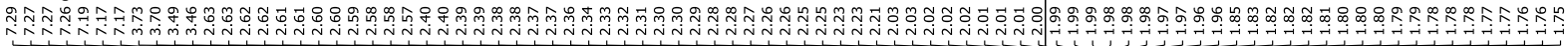
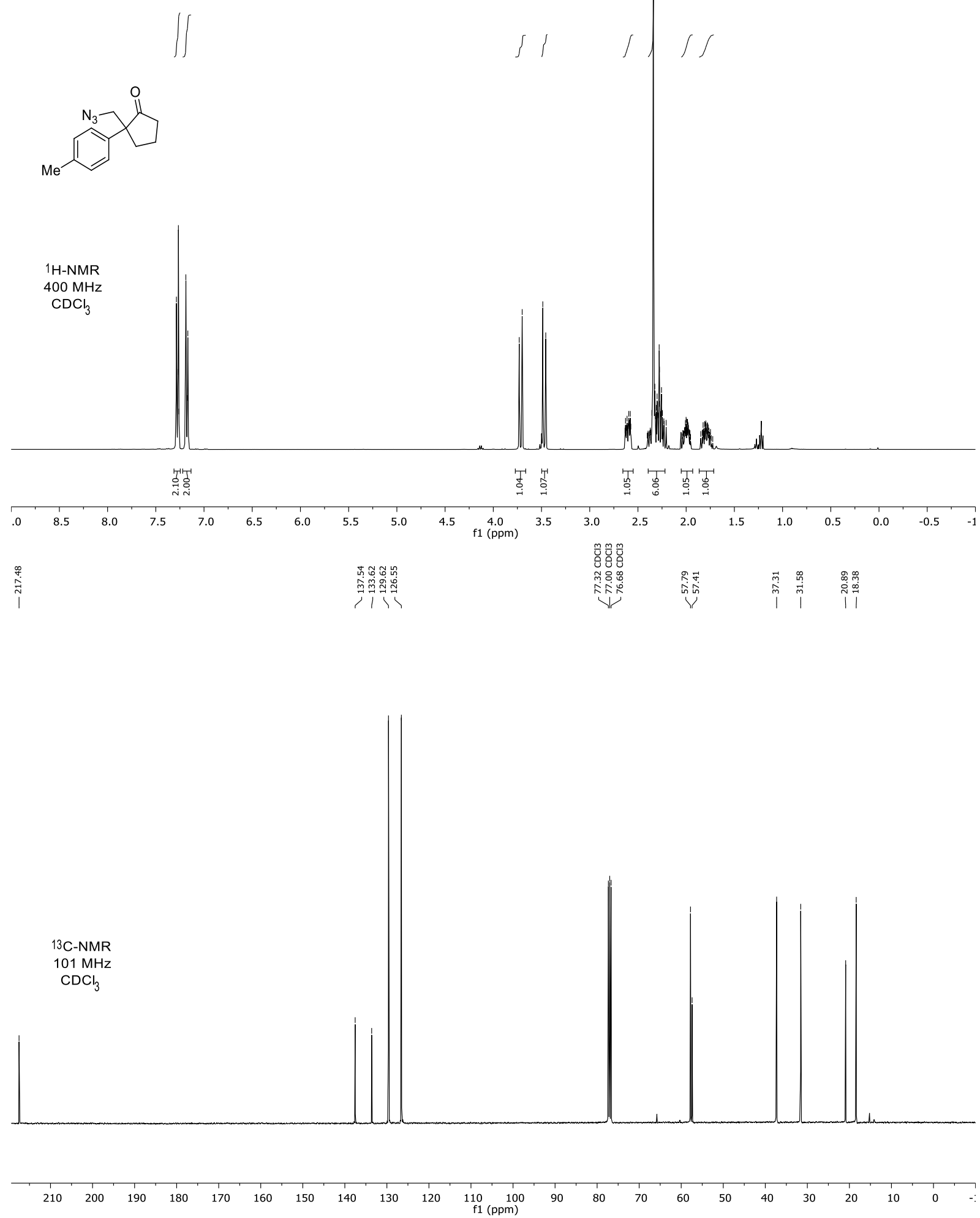
2-(Azidomethyl)-2-(4-(tert-butyl)phenyl)cyclopentanone (6c).

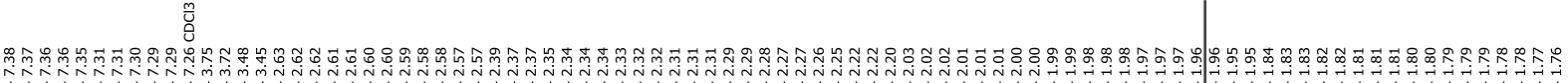

$\iint$

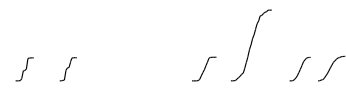<smiles>NCC1(c2ccc(Br)cc2)CCCC1=O</smiles>

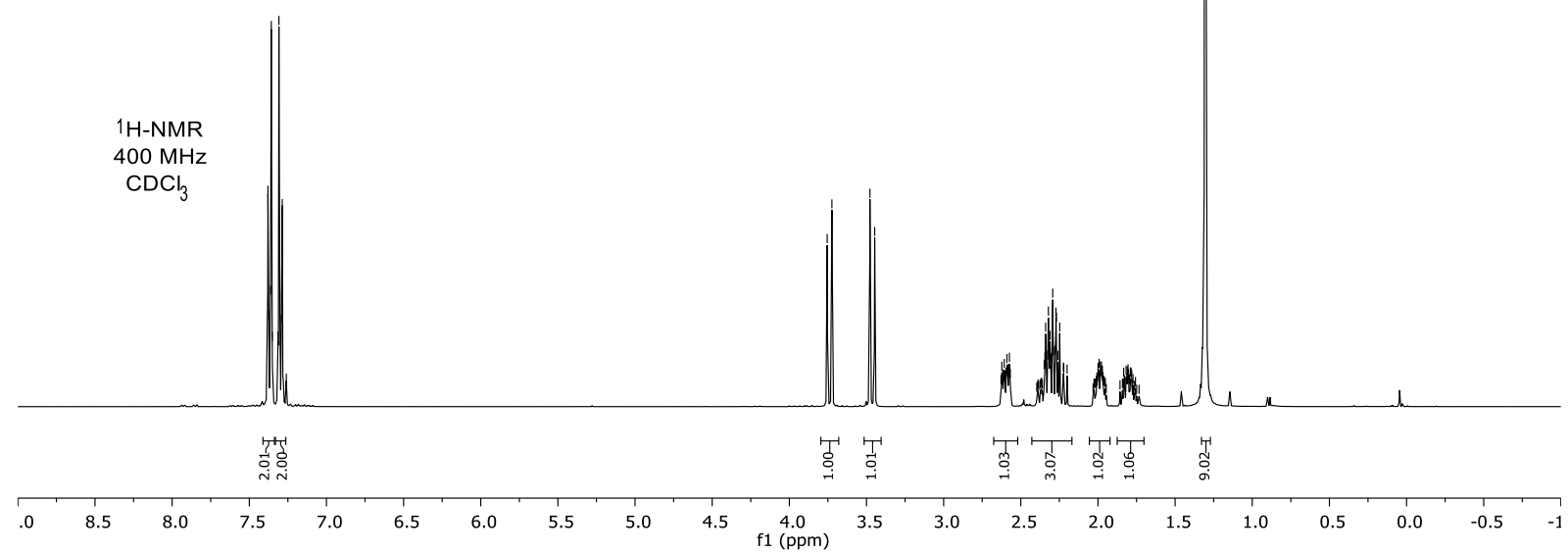

$\stackrel{\text { งิ }}{\stackrel{1}{1}}$

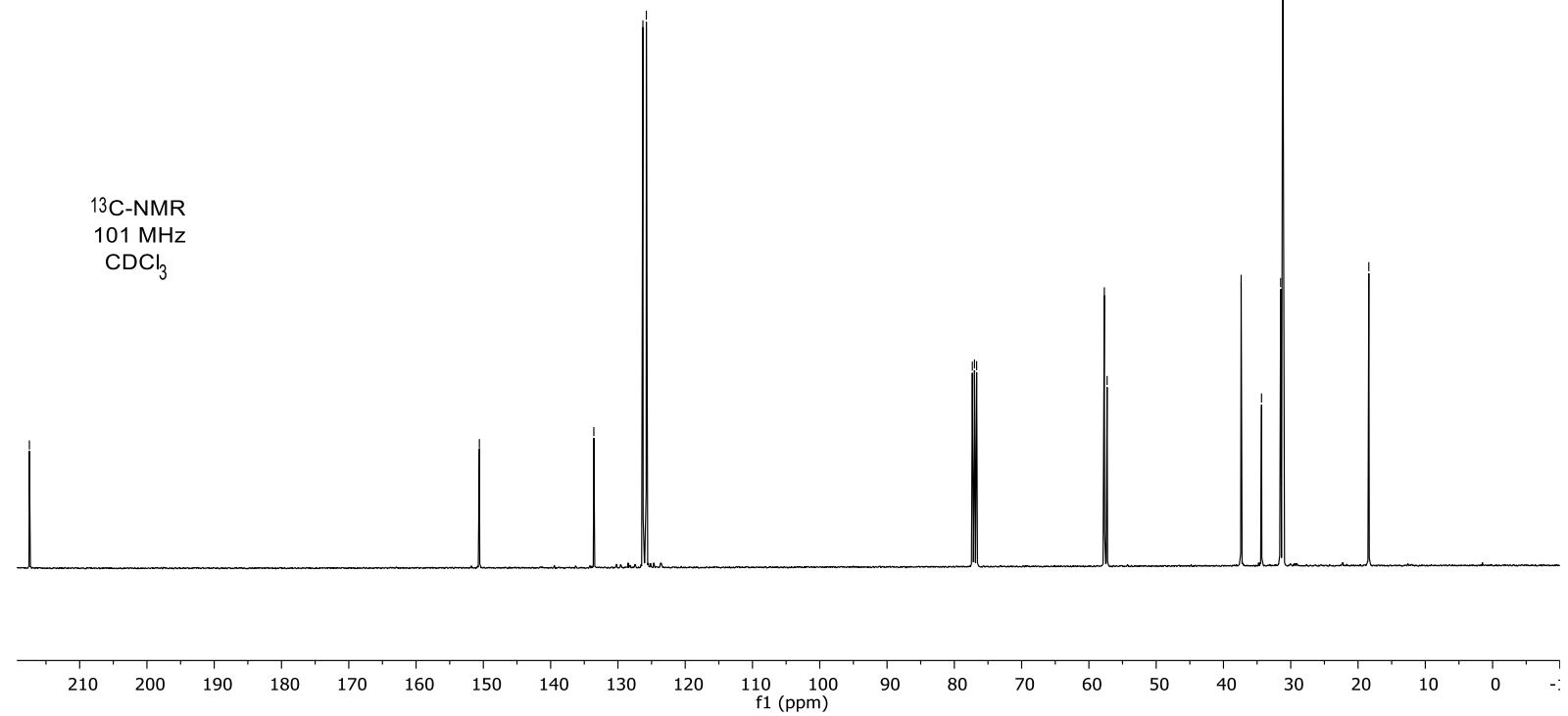


2-([1,1'-Biphenyl]-4-yl)-2-(azidomethyl)cyclopentanone (6d).
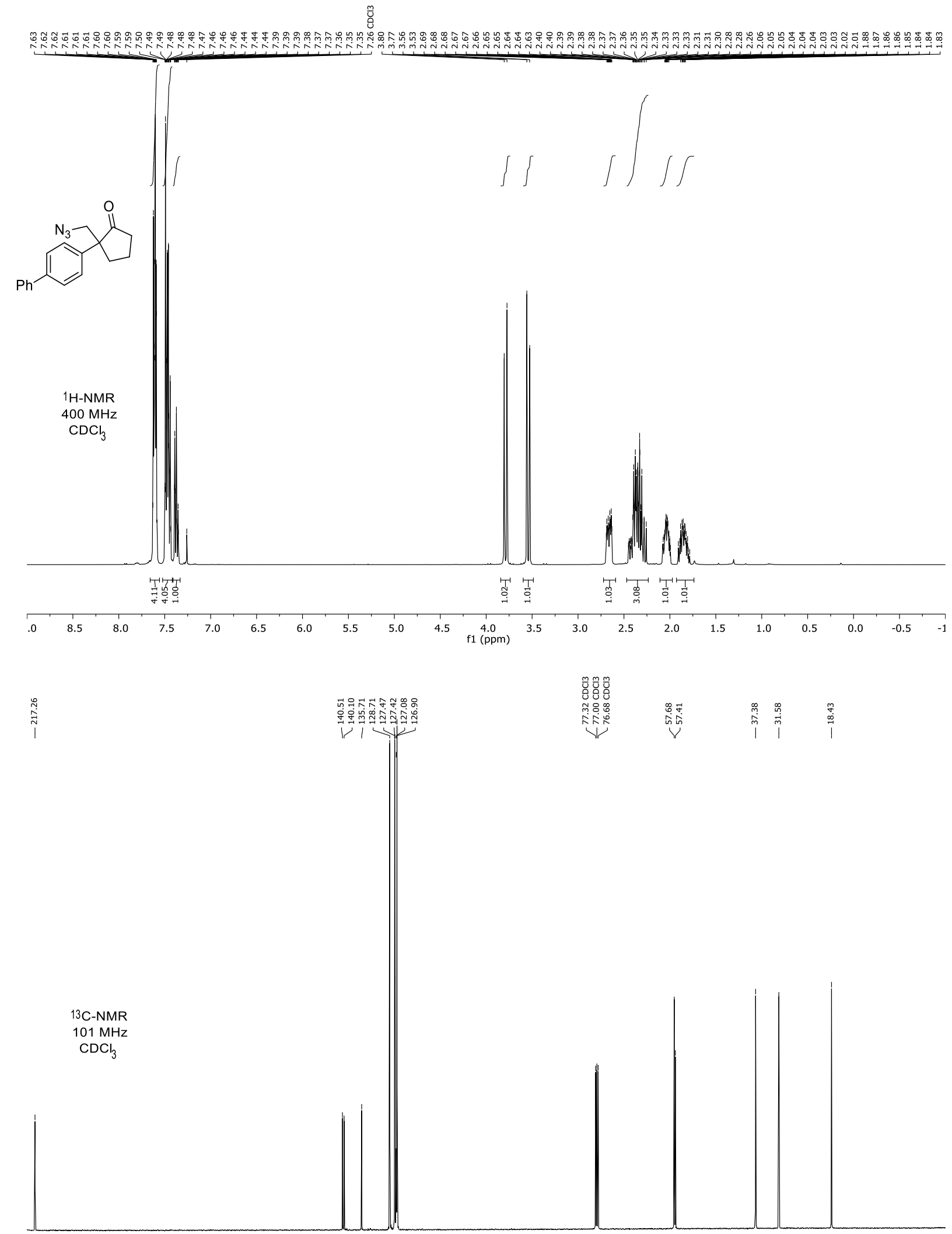

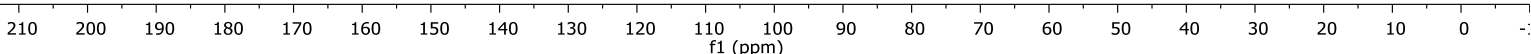


2-(Azidomethyl)-2-(4-methoxyphenyl)cyclopentanone (6e).

突

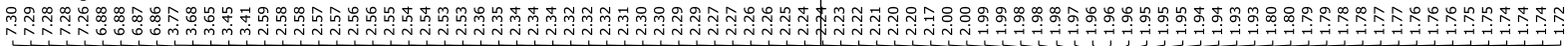

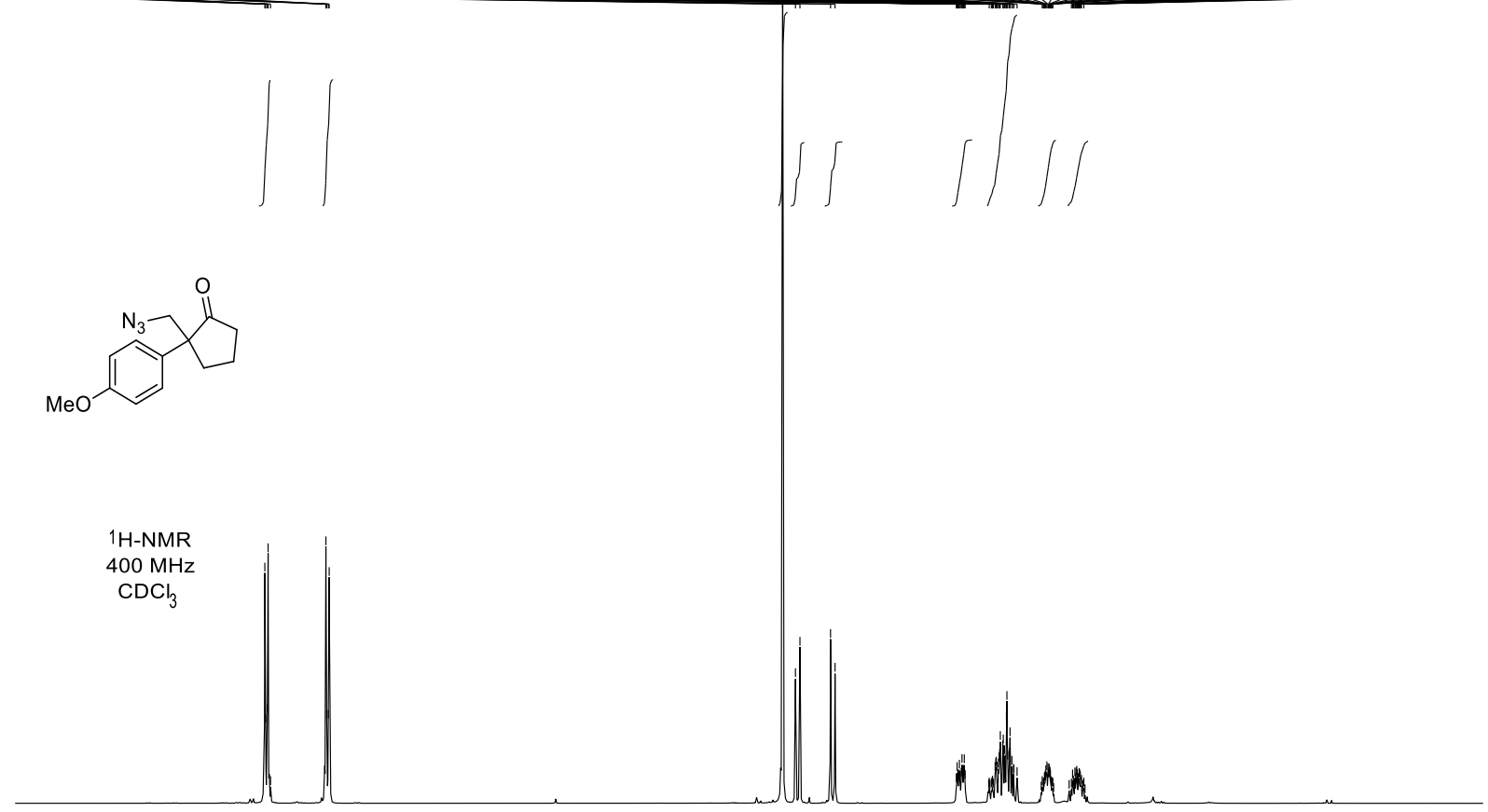

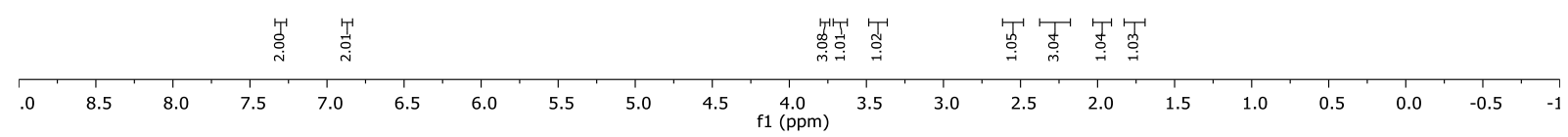

$\stackrel{\substack{\stackrel{N}{N} \\ i}}{i}$

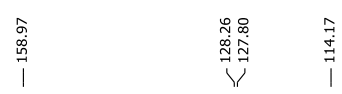

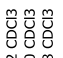

至

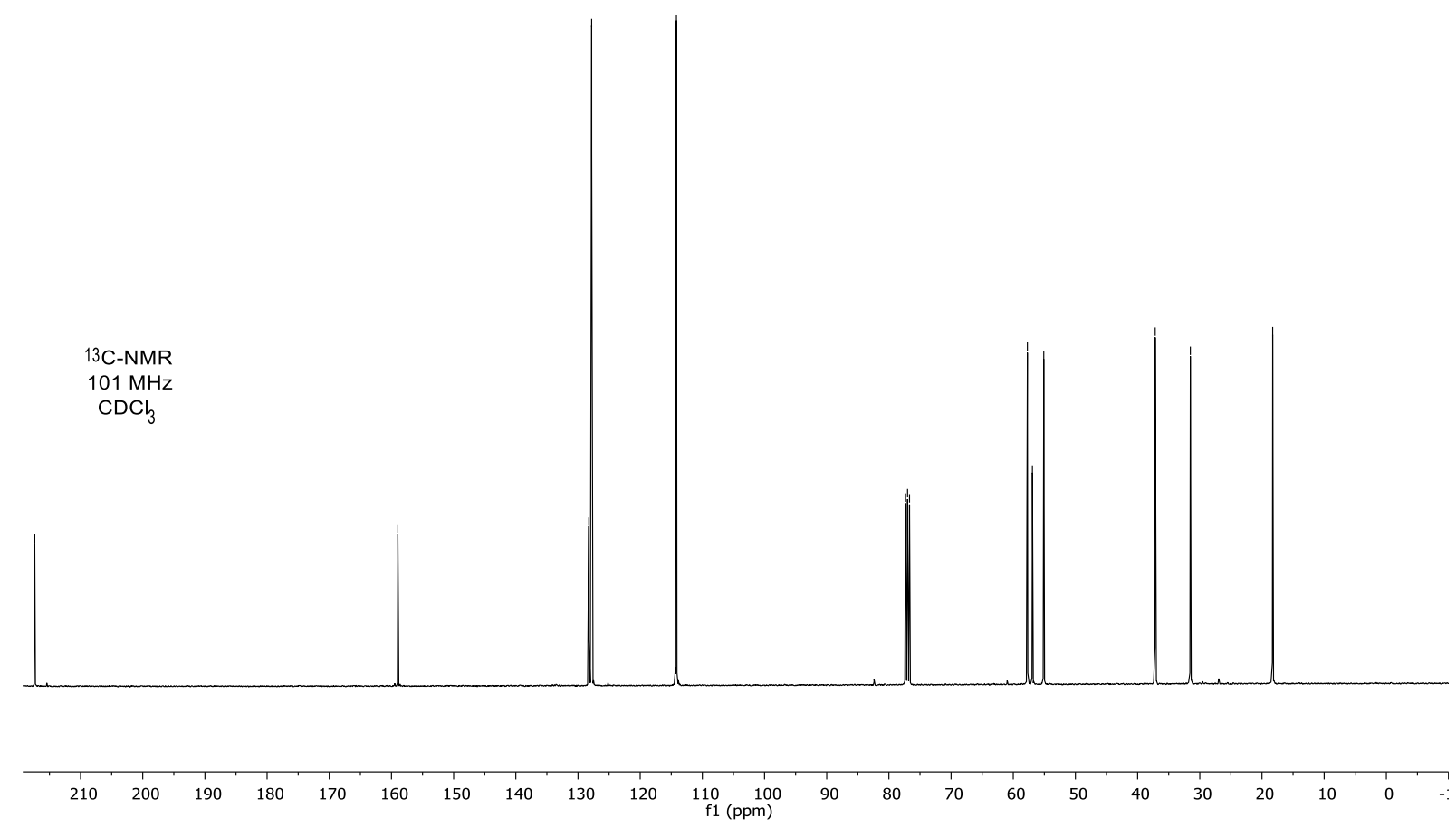


2-(Azidomethyl)-2-(4-fluorophenyl)cyclopentanone (6f)

品

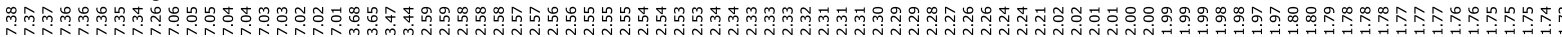

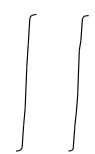

$\iint$

$\iiint$<smiles>O=C1CCCC12CCCNC2</smiles>

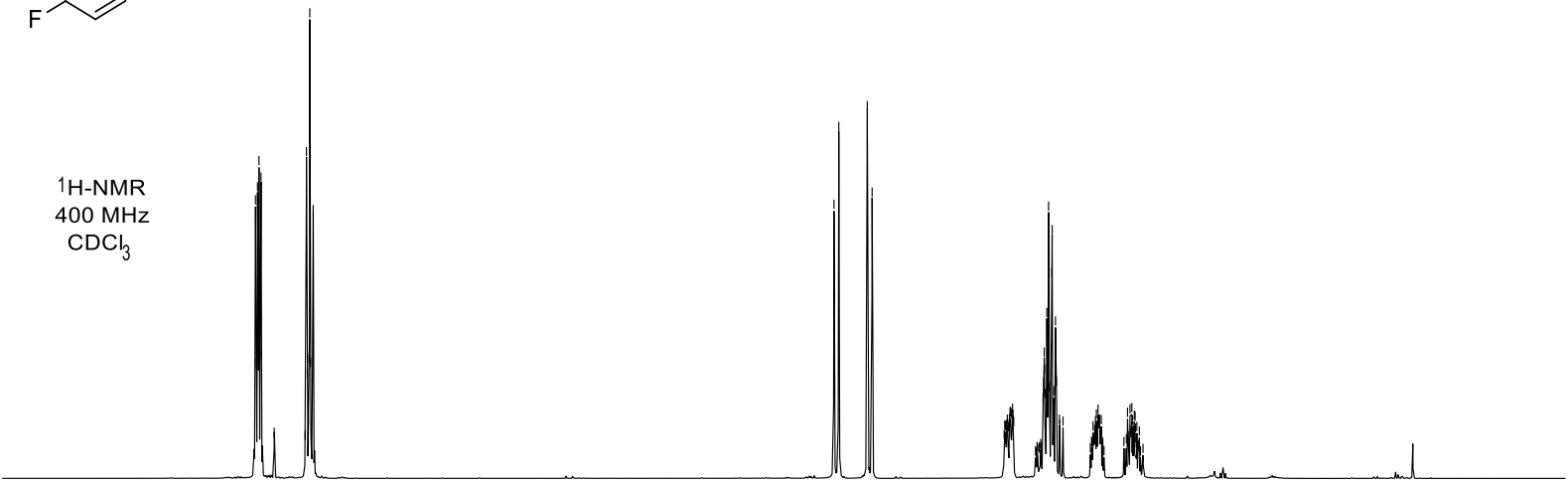

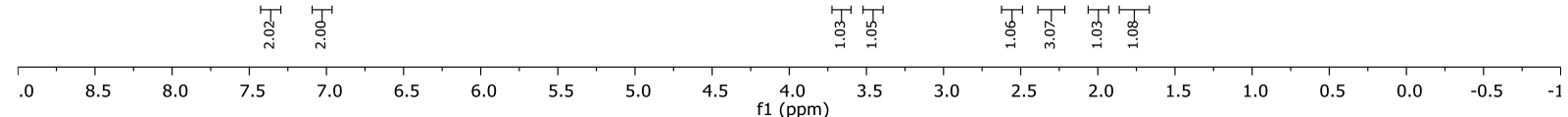

$\stackrel{\hat{n}}{\stackrel{1}{1}}$

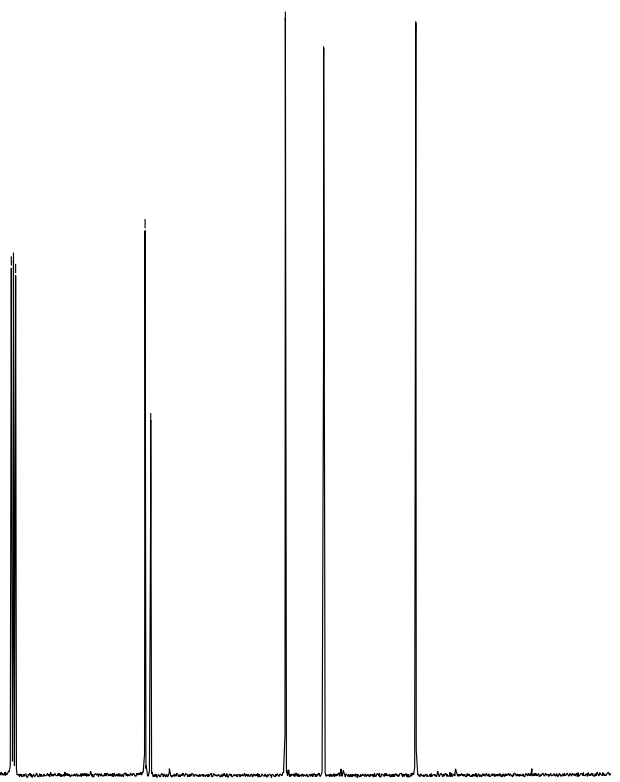

$\begin{array}{lllllllllll}210 & 200 & 190 & 180 & 170 & 160 & 150 & 140 & 130 & 120 & \begin{array}{c}110 \\ \mathrm{f} 1(\mathrm{ppm})\end{array}\end{array}$ 


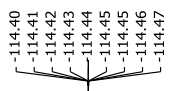

19F-NMR

$376 \mathrm{MHz}$

$\mathrm{CDCl}_{3}$

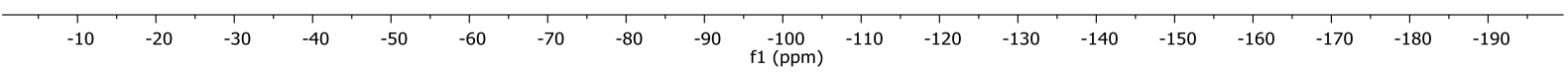

53 
2-(Azidomethyl)-2-(4-chlorophenyl)cyclopentanone (6g).
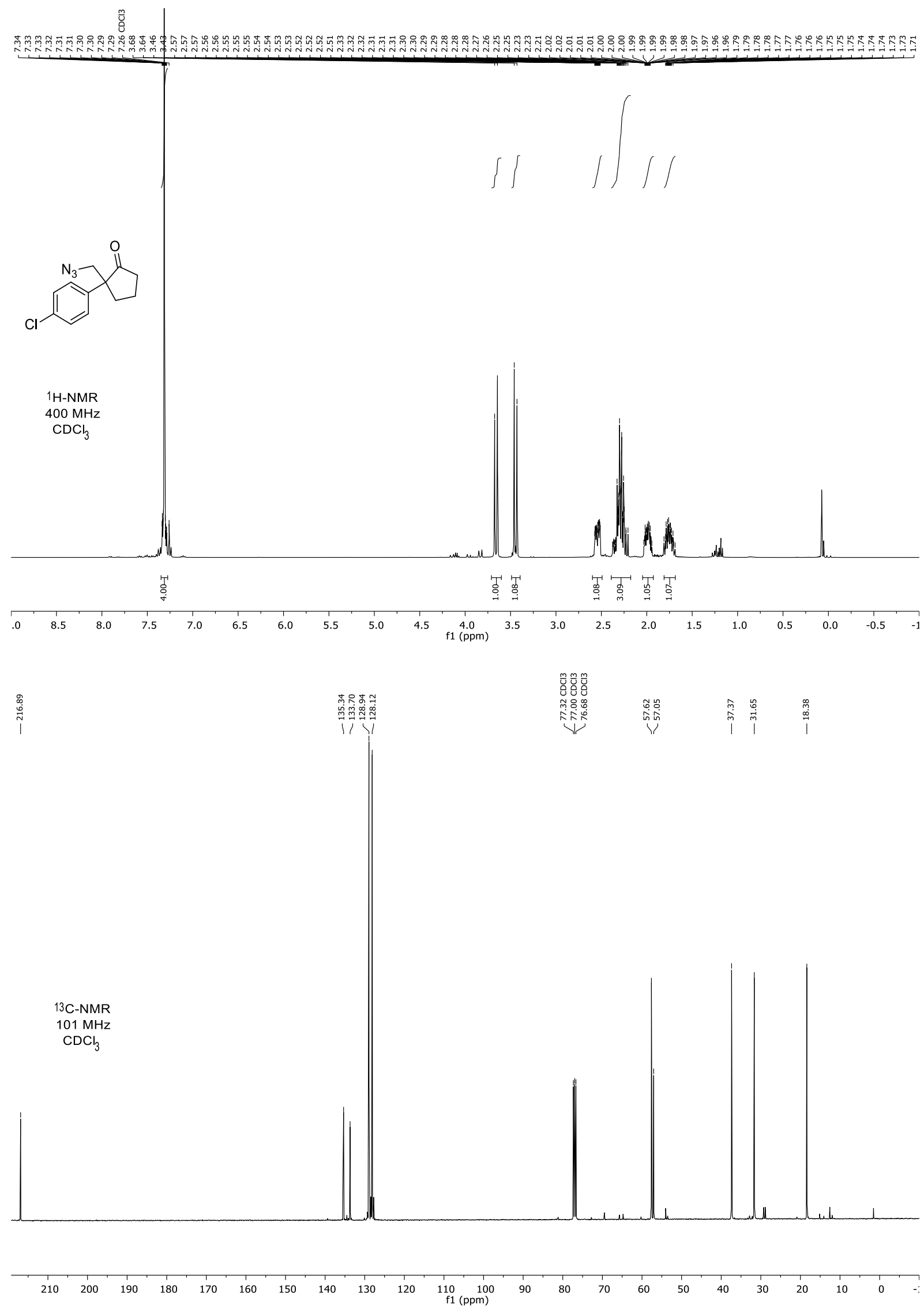
2-(Azidomethyl)-2-(m-tolyl)cyclopentanone (6h).
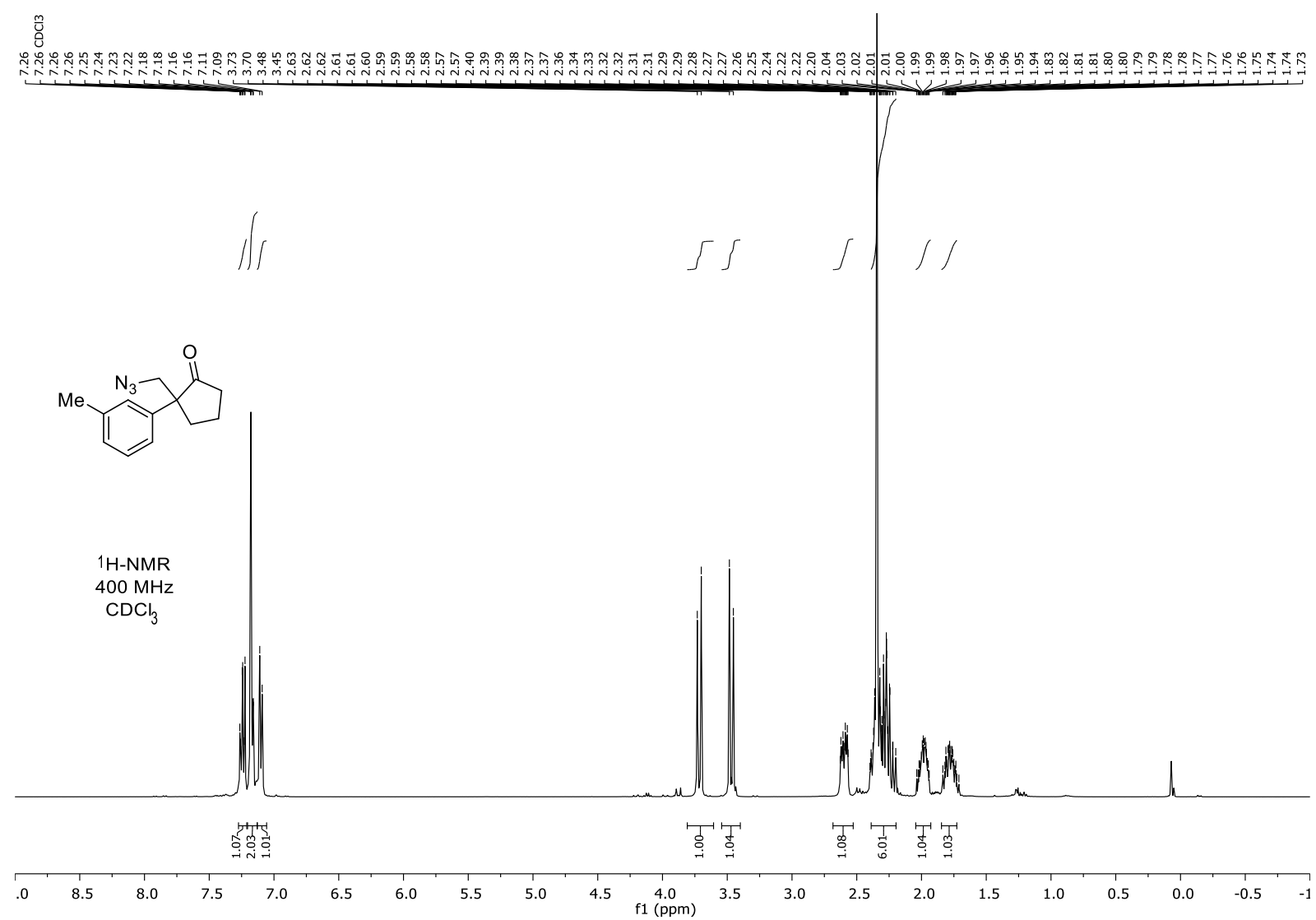

$\stackrel{\text { ิิ }}{\text { ป }}$

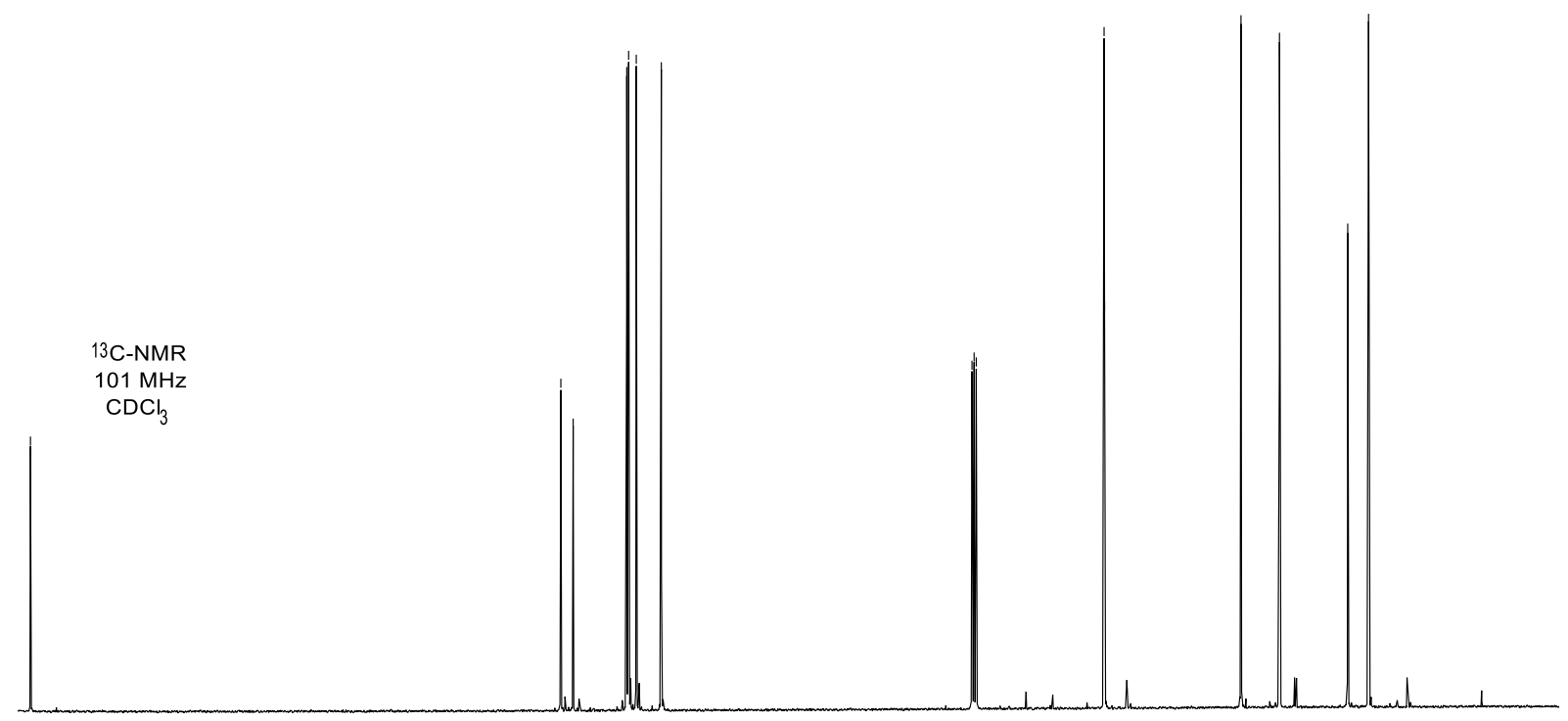

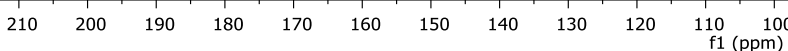


2-(Azidomethyl)-2-(3-methoxyphenyl)cyclopentanone (6i).
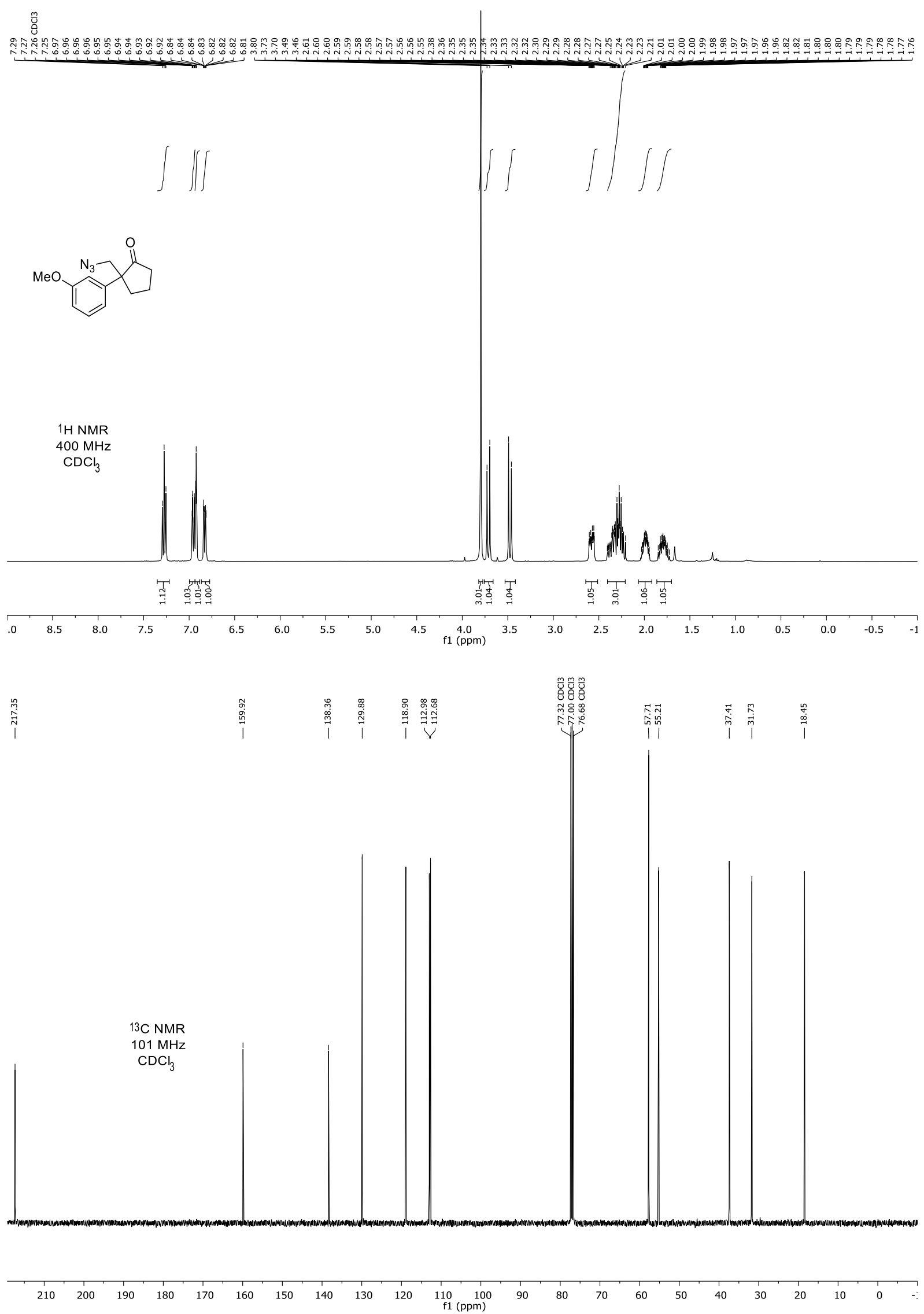

56 
2-(Azidomethyl)-2-(3-fluorophenyl)cyclopentanone (6j).

范

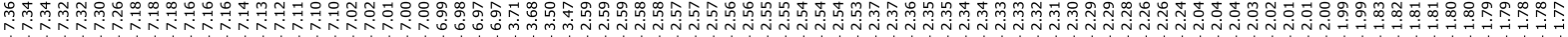
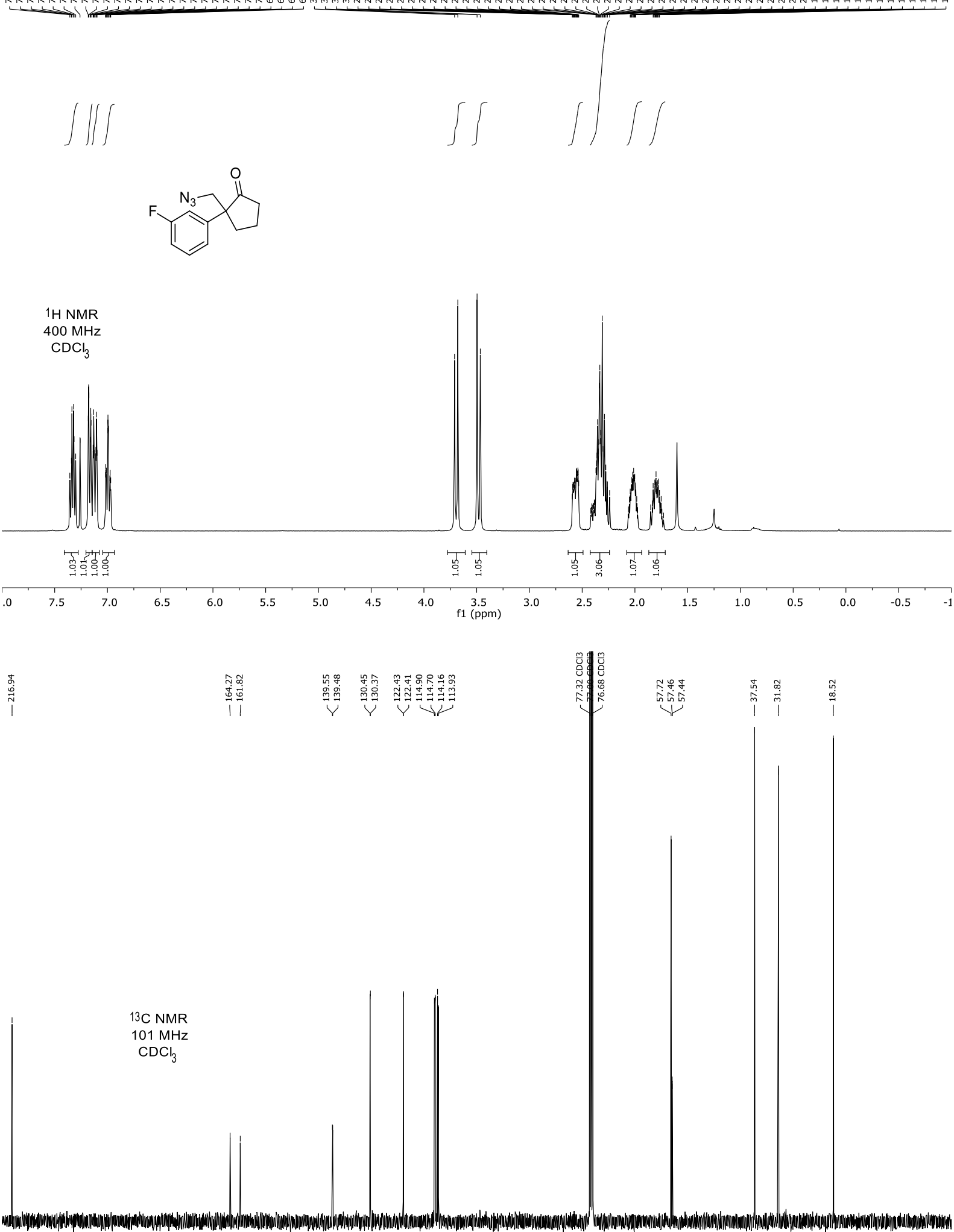

$\begin{array}{lllllllllll}210 & 200 & 190 & 180 & 170 & 160 & 150 & 140 & 130 & 120 & \begin{array}{l}110 \\ \mathrm{f} 1(\mathrm{ppm})\end{array}\end{array}$ 


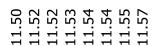

즈를

$19 \mathrm{~F}$ NMR

$376 \mathrm{MHz}$

$\mathrm{CDCl}_{3}$

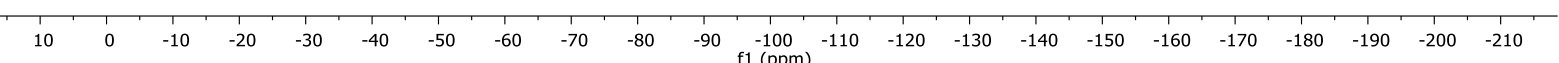


2-(Azidomethyl)-2-(o-tolyl)cyclopentanone (6k).

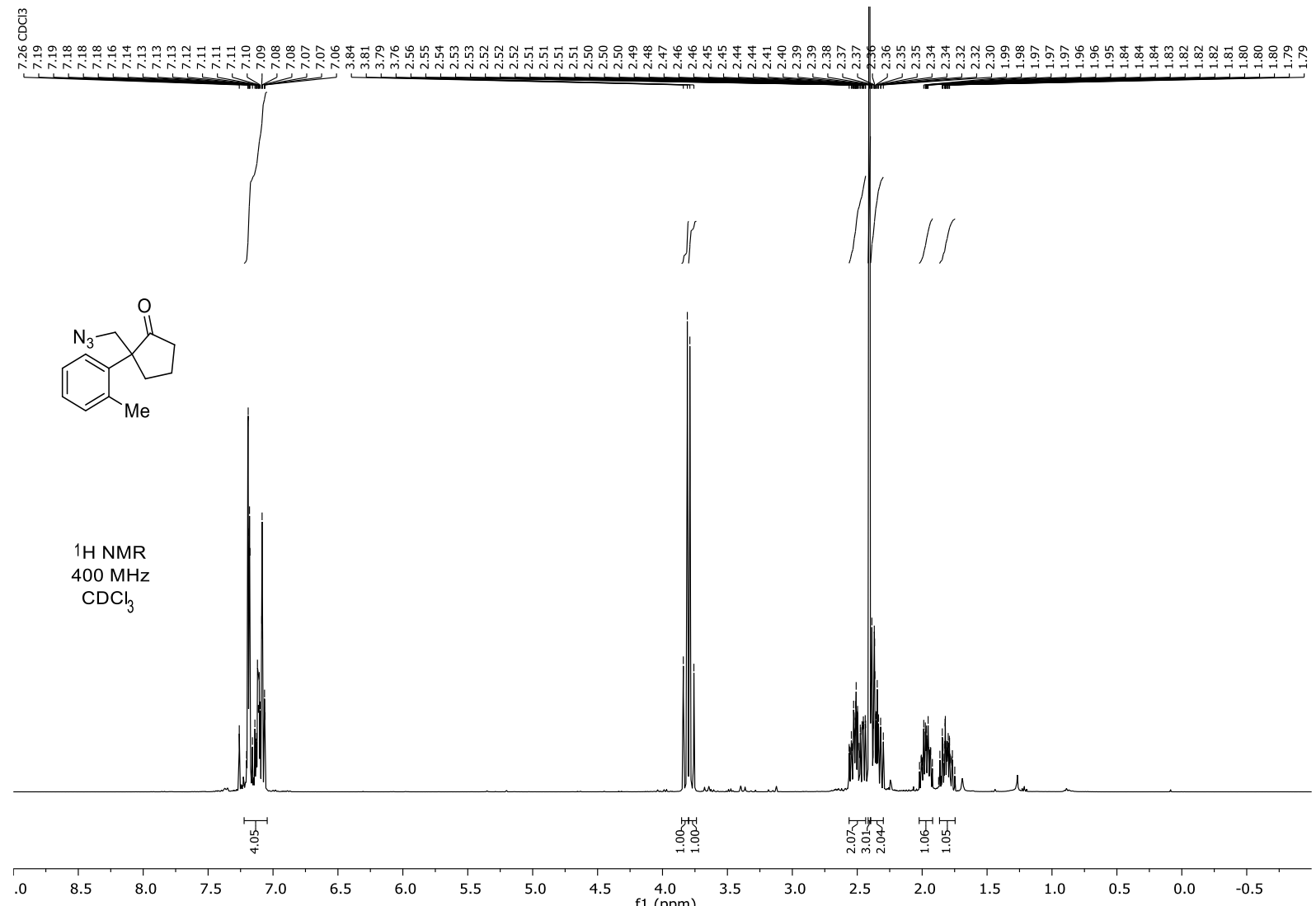

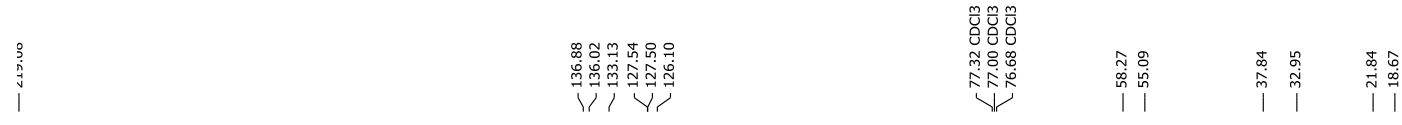

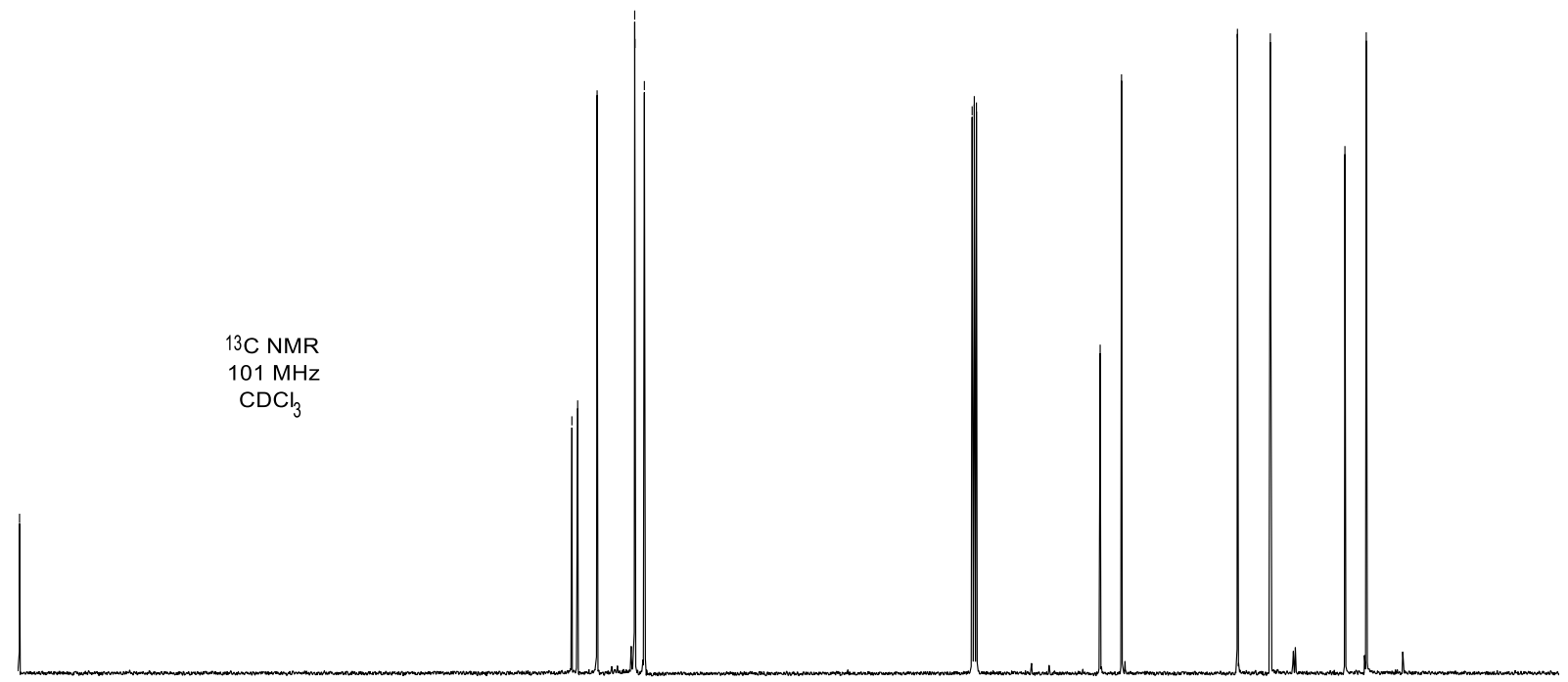

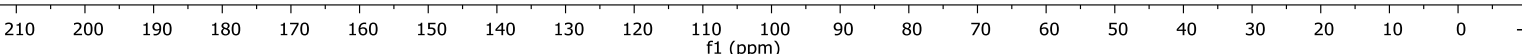


2-(Azidomethyl)-2-(3,4-dimethylphenyl)cyclopentanone (6I).

范 o
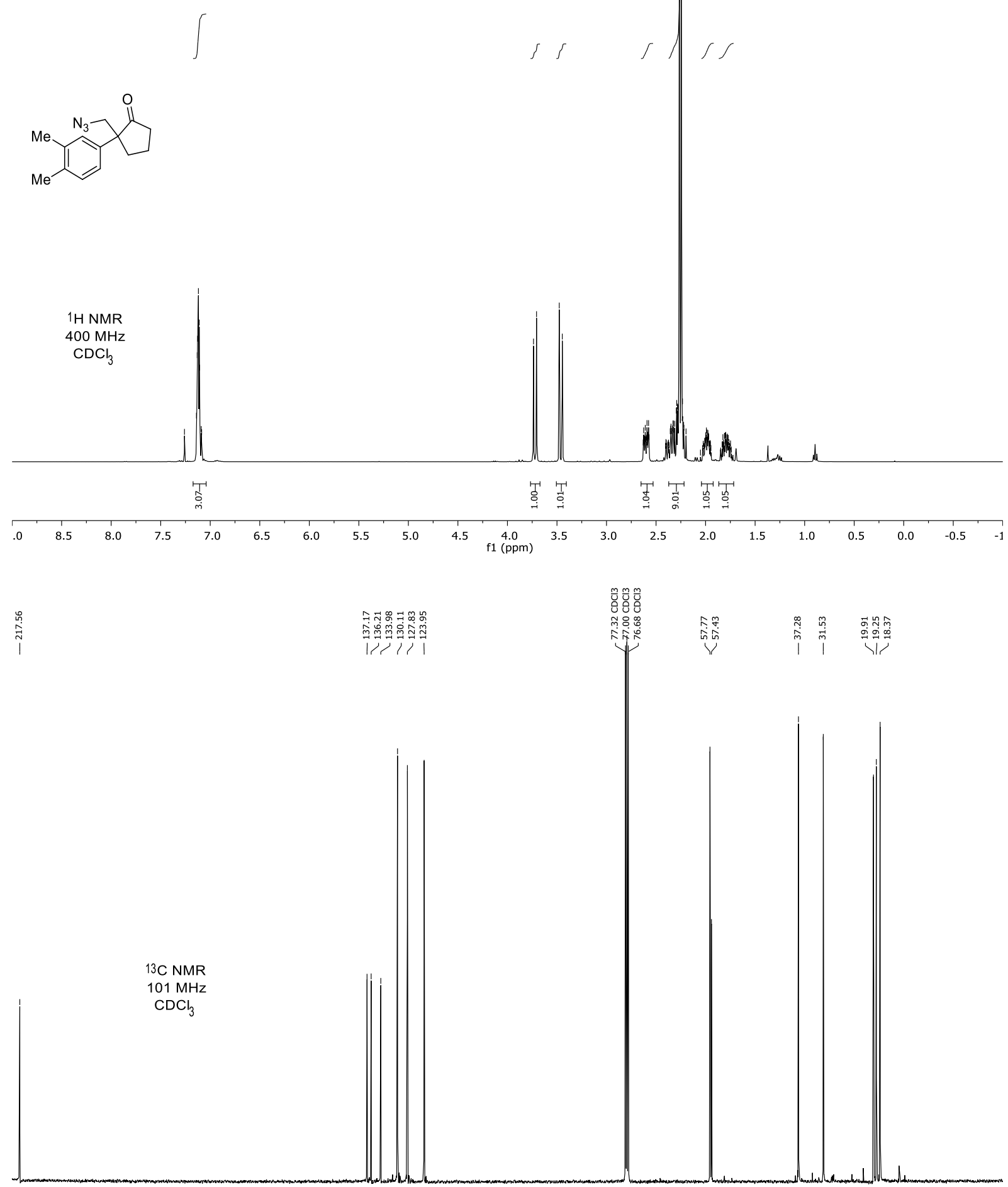

$\begin{array}{lllllllllllllllllllllllll}210 & 200 & 190 & 180 & 170 & 160 & 150 & 140 & 130 & 120 & \underset{\mathrm{f} 1}{110} \underset{(\mathrm{ppm})}{100} & 90 & 80 & 70 & 60 & 50 & 40 & 30 & 20 & 10 & 0 & -\end{array}$ 
2-(Azidomethyl)-2-(3,4-dimethoxyphenyl)cyclopentanone (6m).

芯

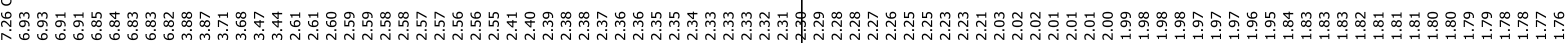

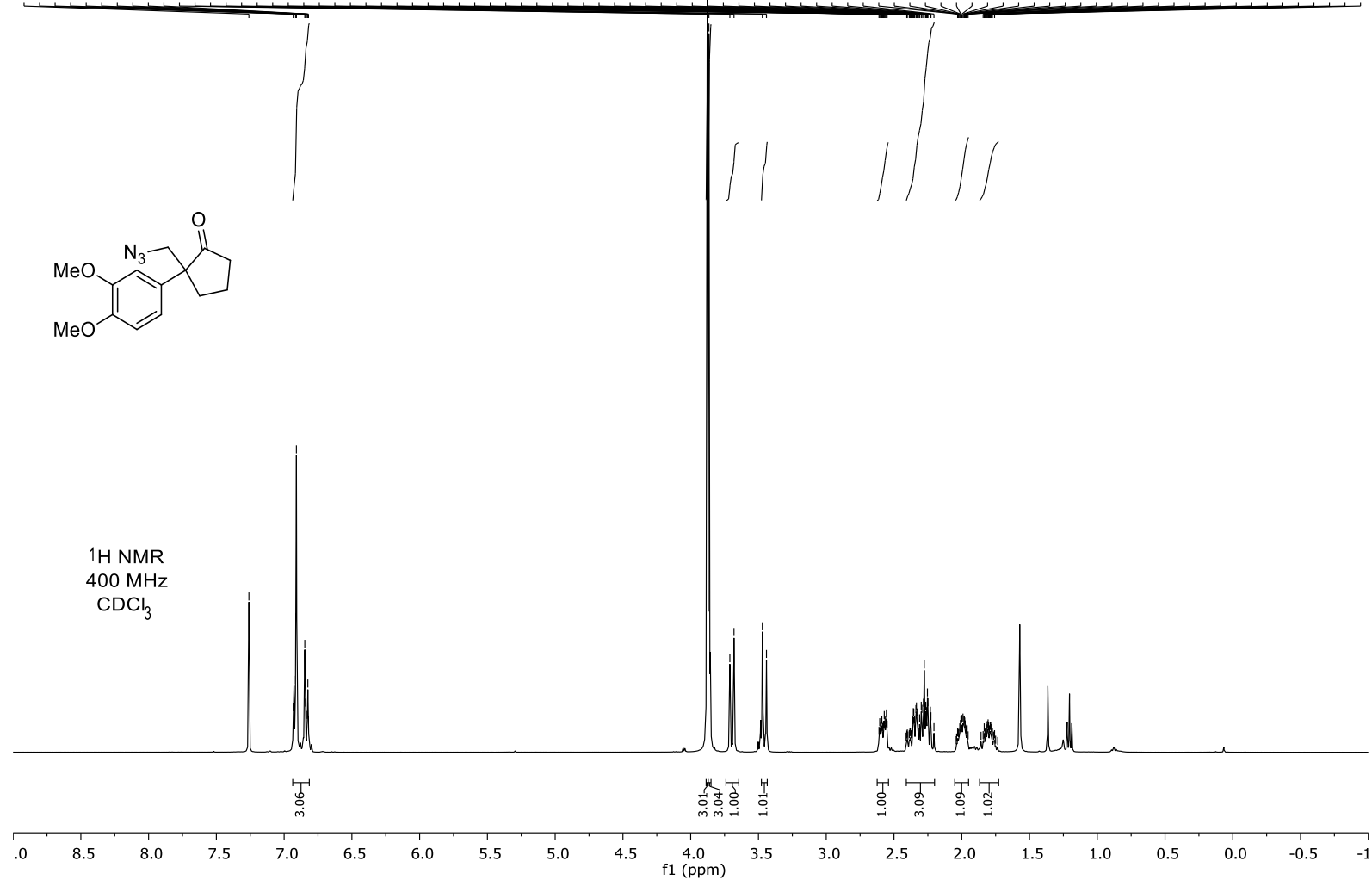

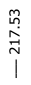

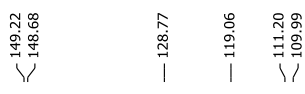

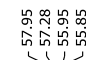

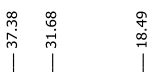

$101 \mathrm{MHz}$

$\mathrm{CDCl}_{3}$

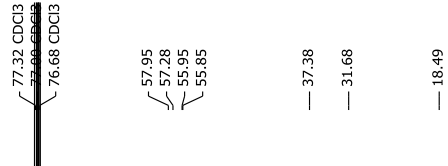

$\begin{array}{lllllllllll}210 & 200 & 190 & 180 & 170 & 160 & 150 & 140 & 130 & 120 & \begin{array}{l}110 \\ \mathrm{f} 1(\mathrm{ppm})\end{array}\end{array}$ 
2-(Azidomethyl)-2-(benzo[d][1,3]dioxol-5-yl)cyclopentanone (6n).

范

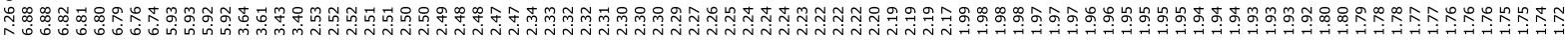

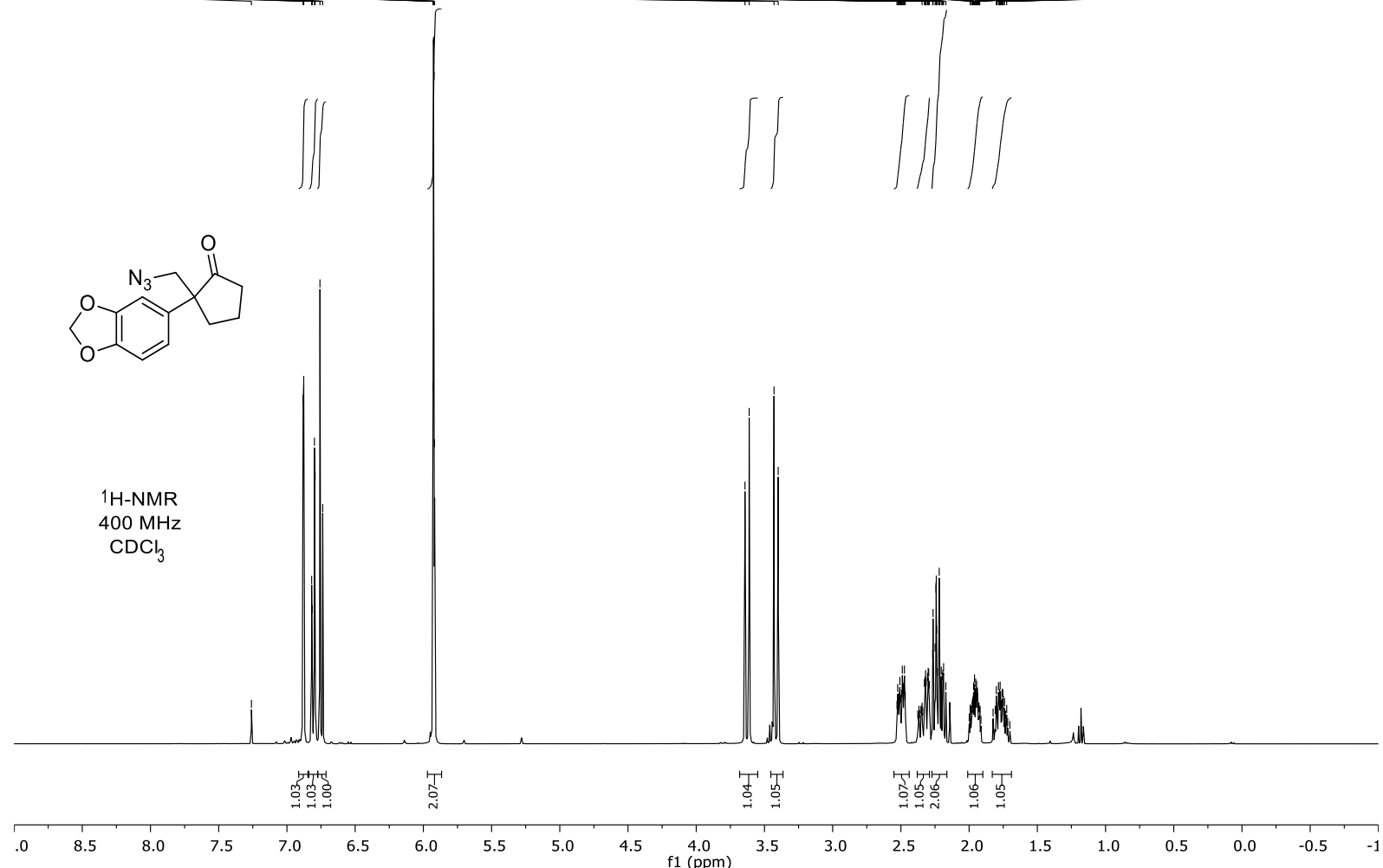

$\stackrel{\text { ำ }}{\stackrel{1}{1}}$

$101 \mathrm{MHz}$

$\mathrm{CDCl}_{3}$
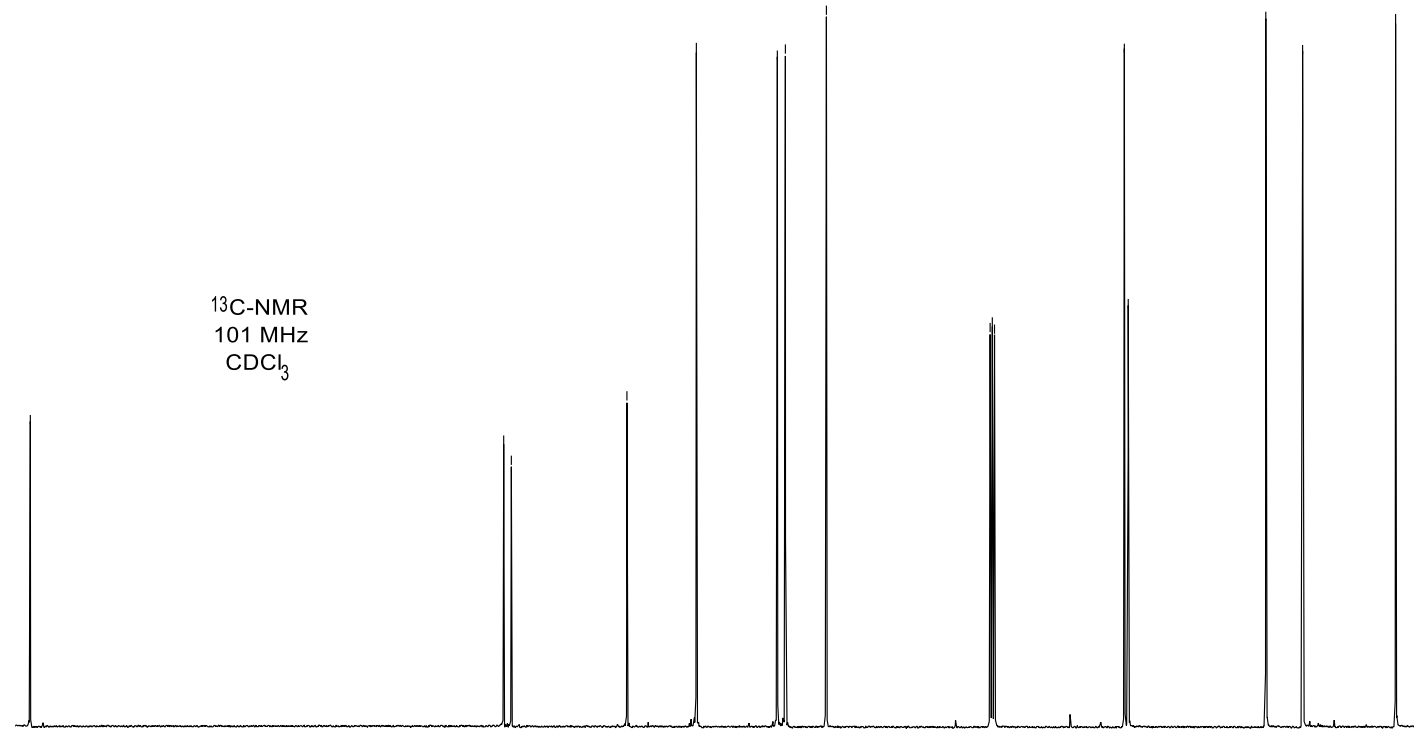

$\begin{array}{lllllllllll}210 & 200 & 190 & 180 & 170 & 160 & 150 & 140 & 130 & 120 & \begin{array}{l}110 \\ \mathrm{f} 1(\mathrm{ppm})\end{array}\end{array}$ 
2-(Azidomethyl)-2-(naphthalen-2-yl)cyclopentanone (6o).

愛
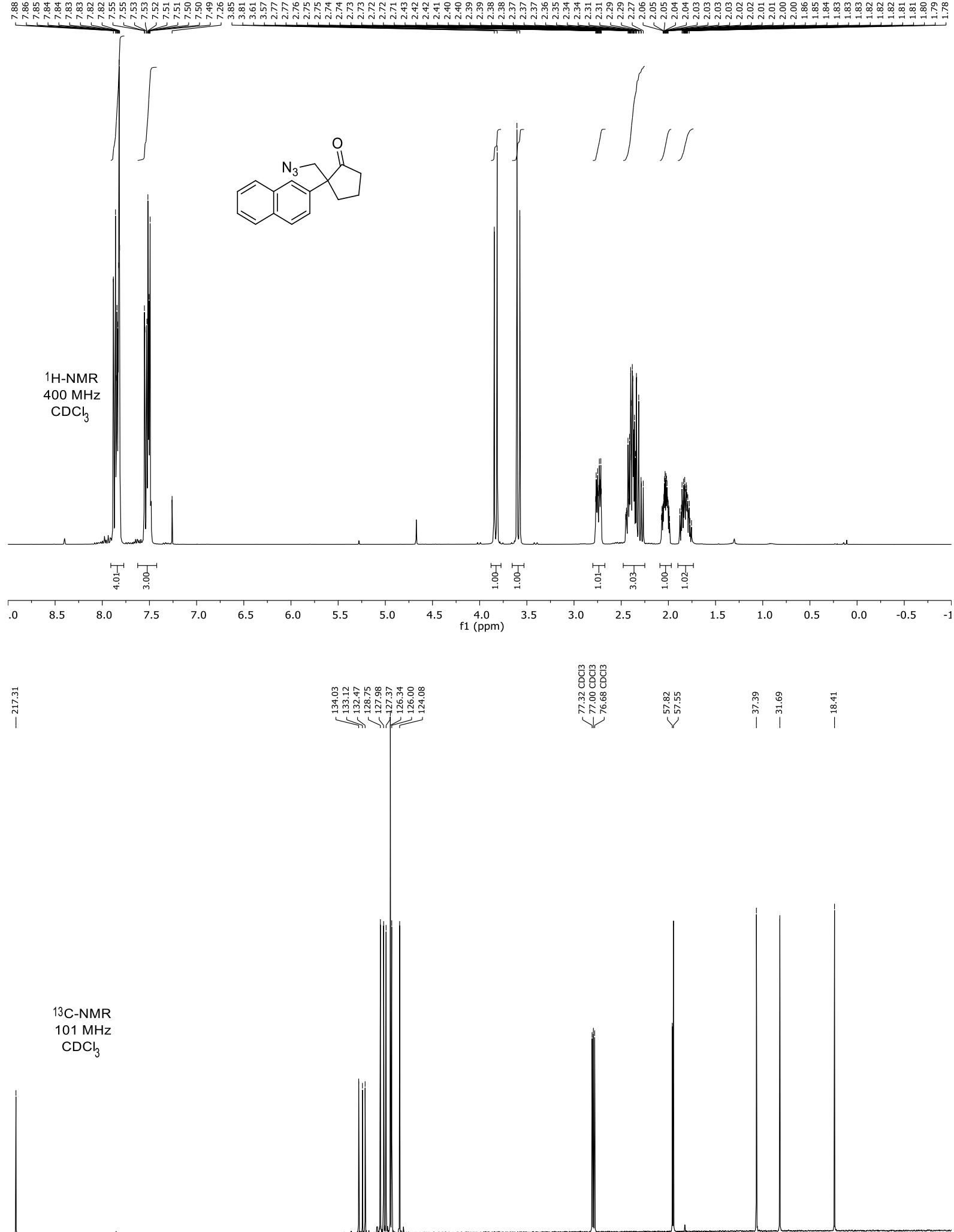

$\begin{array}{llllllllllllllllllllllllllllll}210 & 200 & 190 & 180 & 170 & 160 & 150 & 140 & 130 & 120 & 110 & 100 & 90 & 80 & 70 & 60 & 50 & 40 & 30 & 20 & 10 & 0 & -\end{array}$ 
2-(Azidomethyl)-2-(naphthalen-1-yl)cyclopentanone (6p).
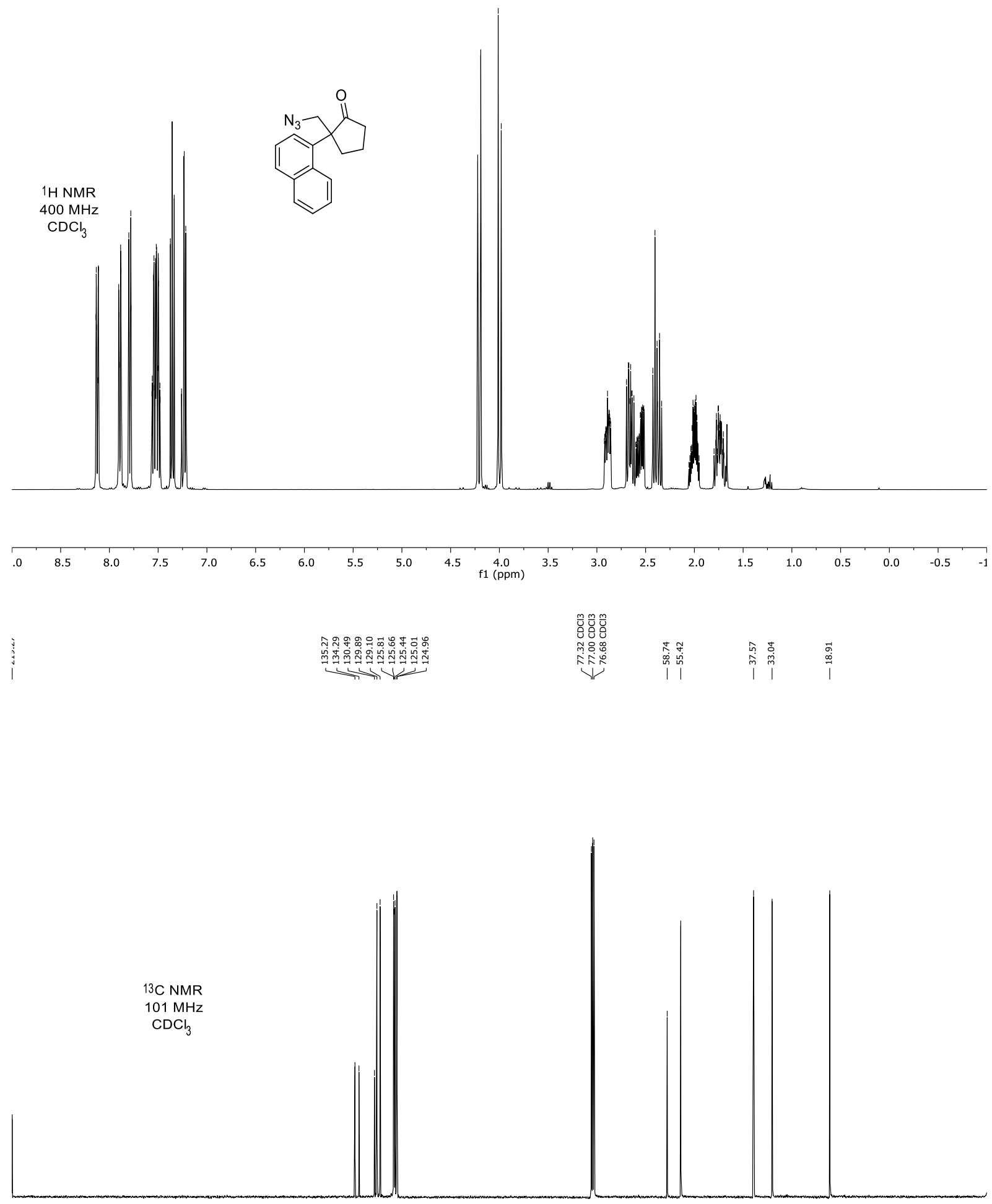

$\begin{array}{llllllllllllllllllllllllllll}210 & 200 & 190 & 180 & 170 & 160 & 150 & 140 & 130 & 120 & 110 & 100 & 90 & 80 & 70 & 60 & 50 & 40 & 30 & 20 & 10 & 0 & -10\end{array}$ 
4-(Azidomethyl)-4-phenyldihydrofuran-3(2H)-one (6q).
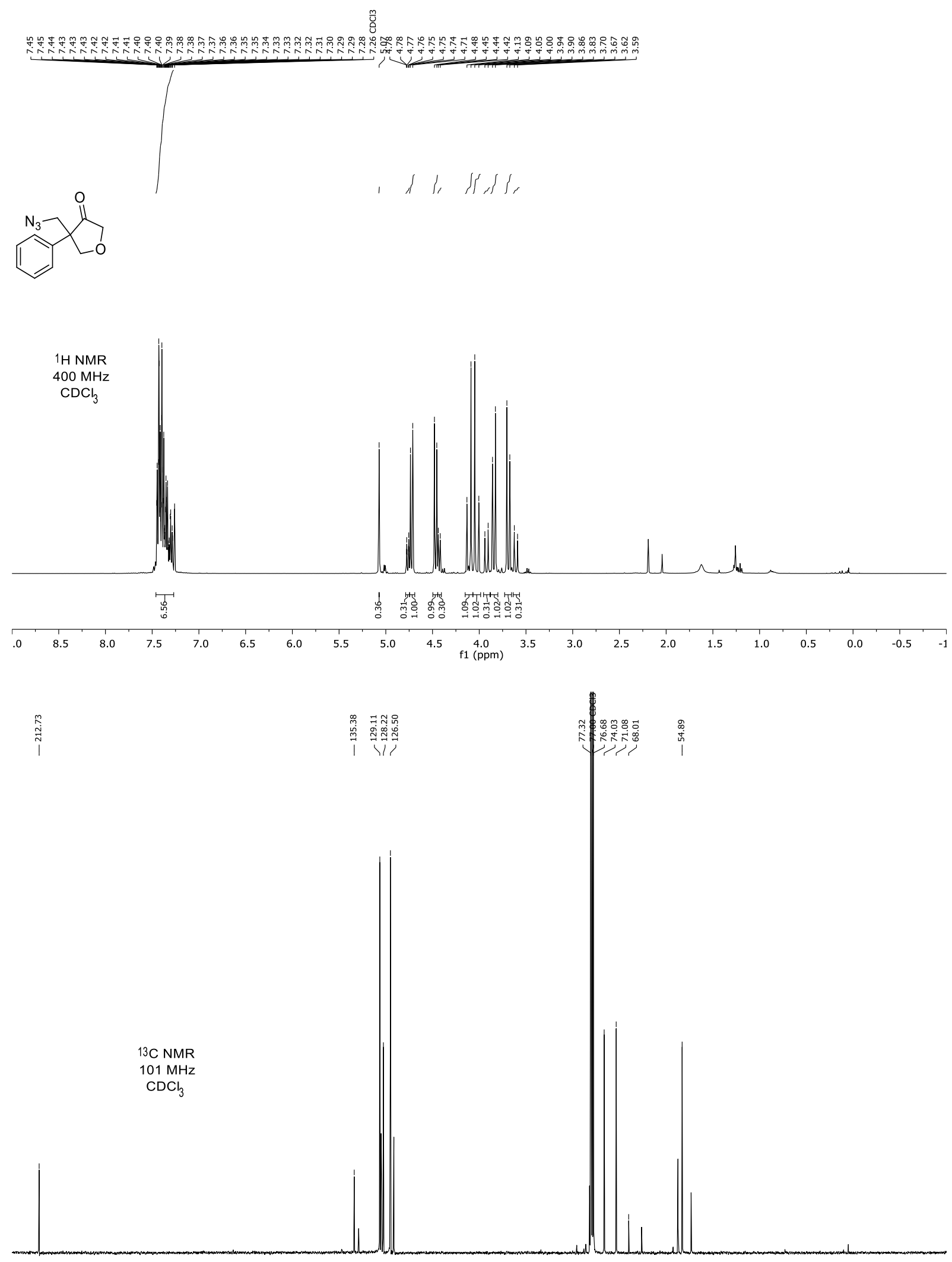

$\begin{array}{llllllllllllllllllllllll}210 & 200 & 190 & 180 & 170 & 160 & 150 & 140 & 130 & 120 & \underset{\mathrm{f} 1}{110}(\mathrm{ppm}) & 100 & 90 & 80 & 70 & 60 & 50 & 40 & 30 & 20 & 10 & 0 & -\end{array}$ 
2-(Azidomethyl)-2-(4-(trifluoromethyl)phenyl)-1-oxaspiro[2.3]hexane (7t).

范

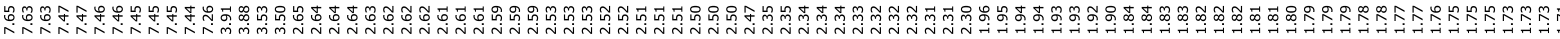
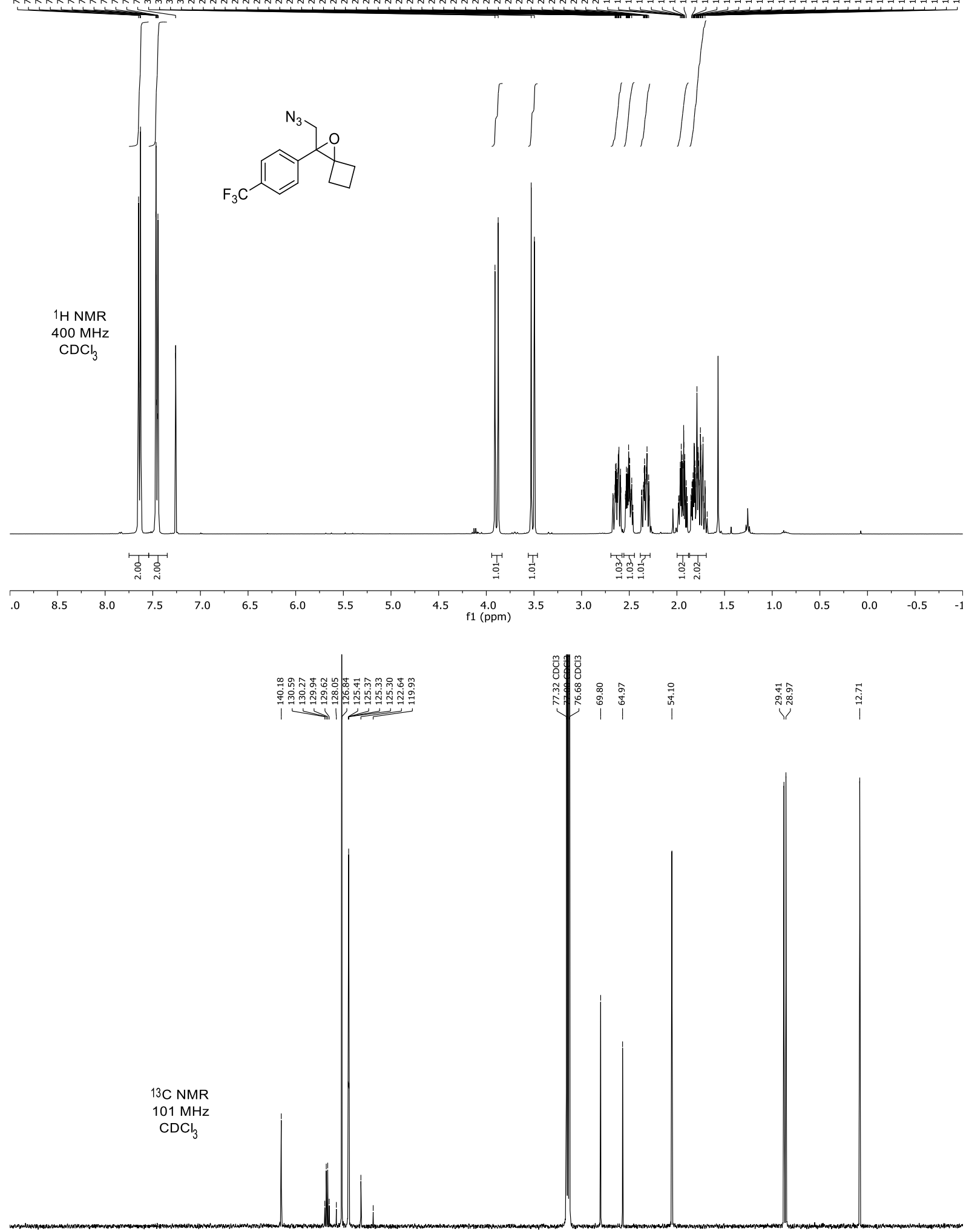

$\begin{array}{lllllllllll}\text { D0 } & 190 & 180 & 170 & 160 & 150 & 140 & 130 & 120 & 110 & 100 \\ \text { f1 (ppm) }\end{array}$ 


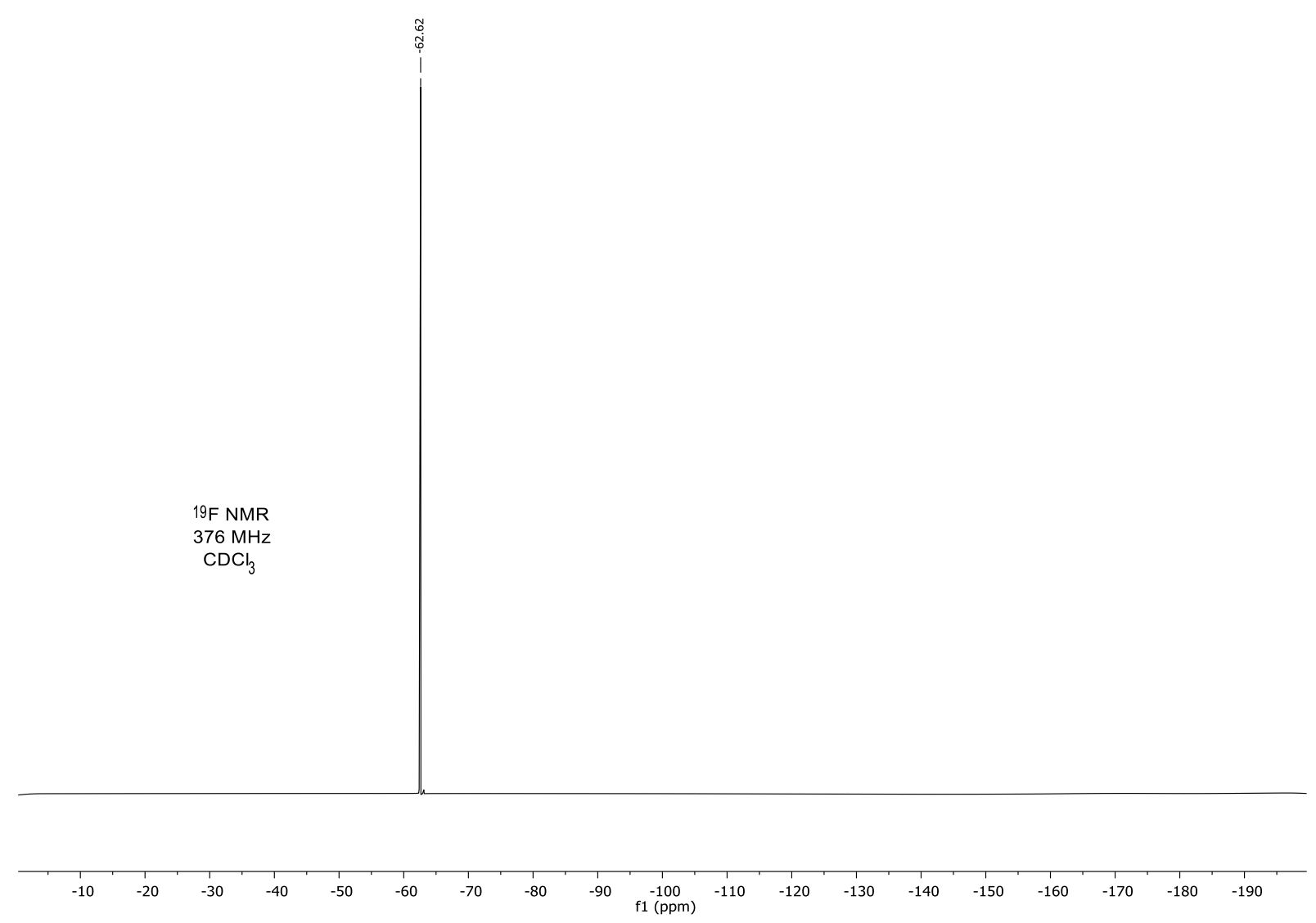


2-Phenyl-2-((4-phenyl-1H-1,2,3-triazol-1-yl)methyl)cyclopentanone (14).

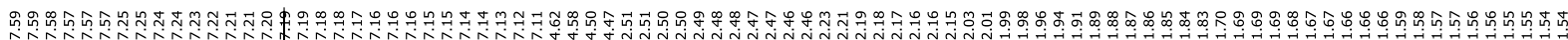
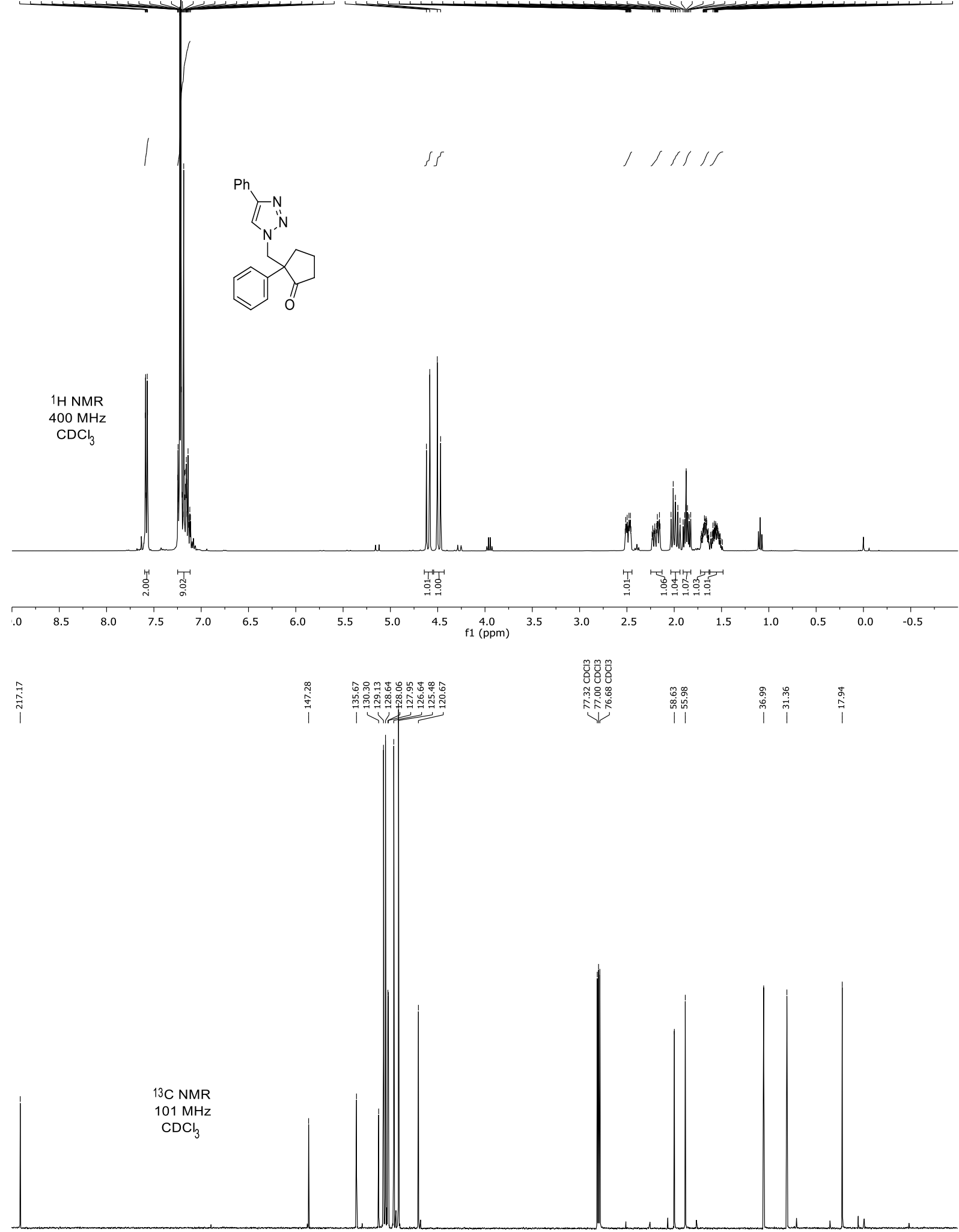

$\begin{array}{lllllllllll}210 & 200 & 190 & 180 & 170 & 160 & 150 & 140 & 130 & 120 & 110 \\ \mathrm{f} 1(\mathrm{ppm})\end{array}$
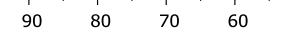
Benzyl ((2-oxo-1-phenylcyclopentyl)methyl)carbamate (15).
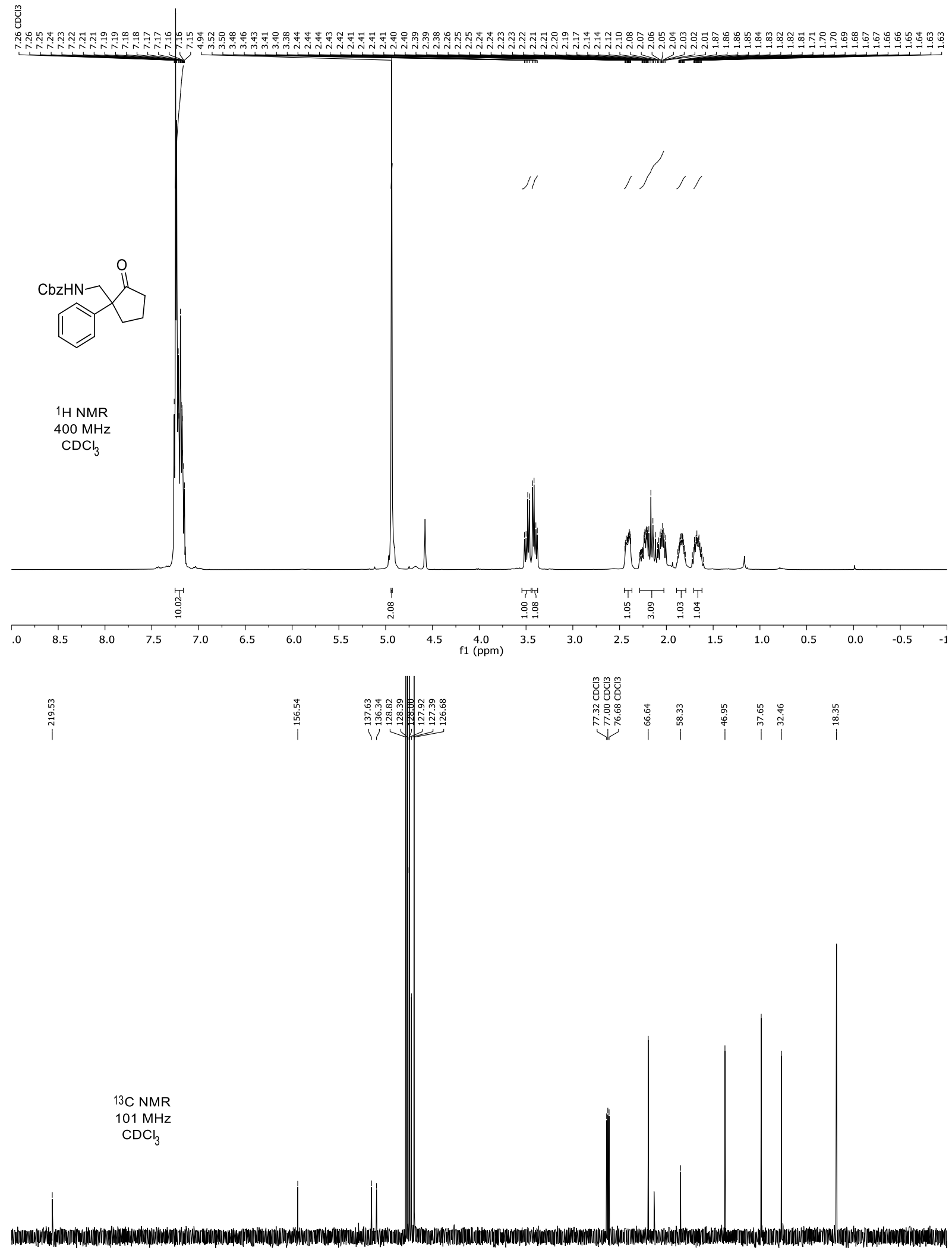

$\begin{array}{llllllllllllllllllllllllllllllll}30 & 220 & 210 & 200 & 190 & 180 & 170 & 160 & 150 & 140 & 130 & 120 & 110 & 100 & 90 & 80 & 70 & 60 & 50 & 40 & 30 & 20 & 10 & 0 & -\end{array}$ 
2-(Isothiocyanatomethyl)-2-phenylcyclopentanone (16).

离

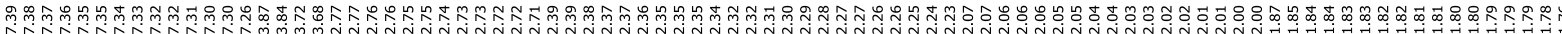

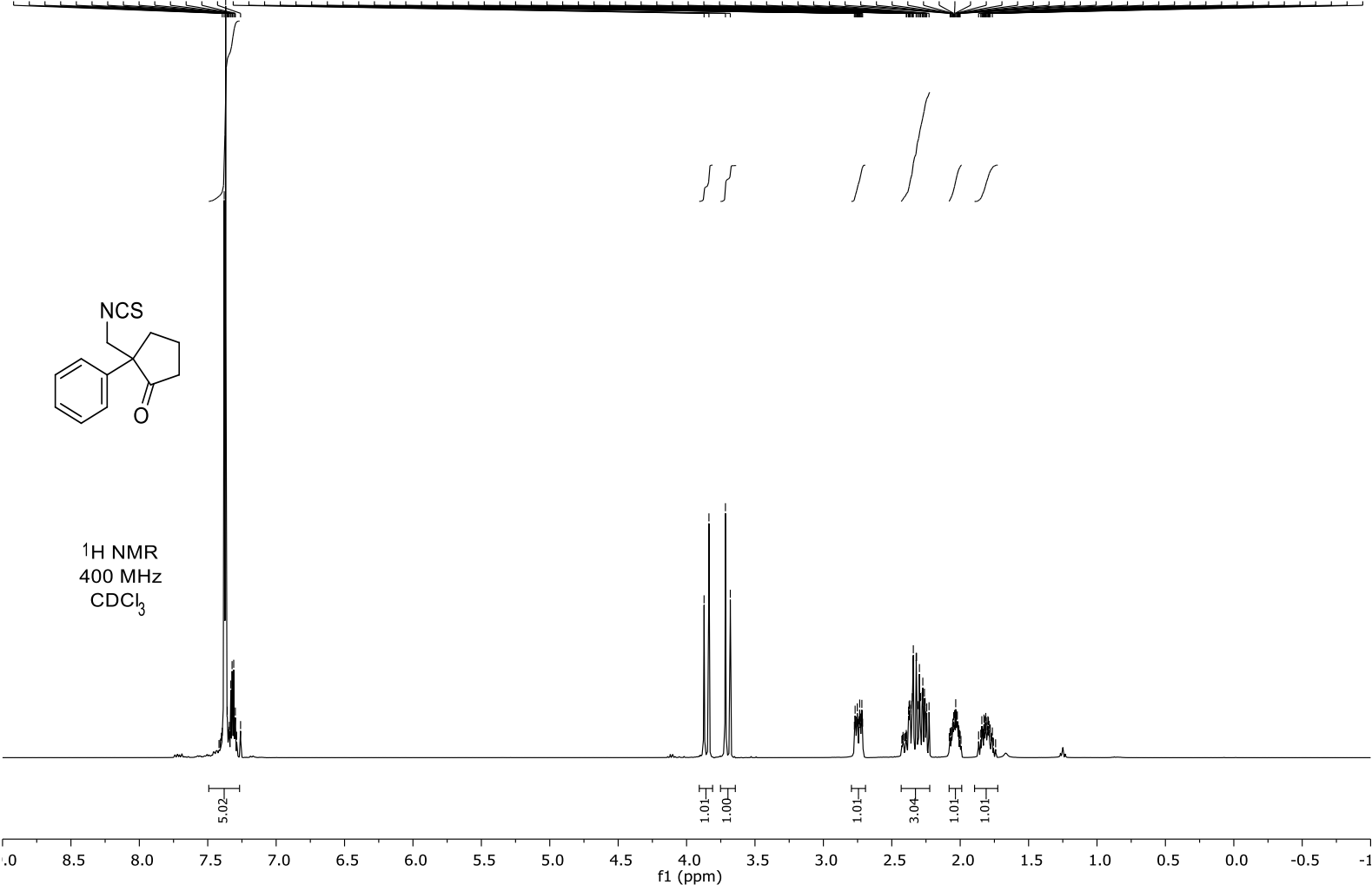

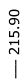

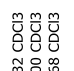

许

$13 \mathrm{C}$ NMR

$101 \mathrm{MHz}$

$\mathrm{CDCl}_{3}$

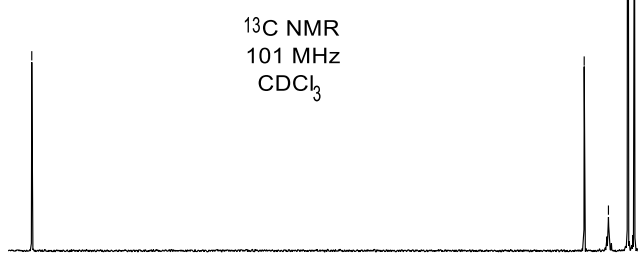

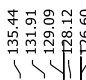

$\begin{array}{lllllllllll}210 & 200 & 190 & 180 & 170 & 160 & 150 & 140 & 130 & 120 & 110 \\ \mathrm{f} 1(\mathrm{ppm}) & 100\end{array}$

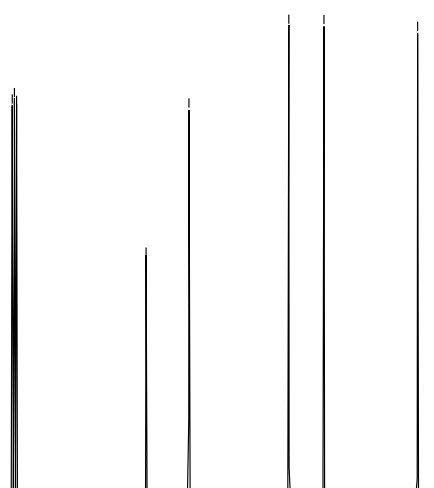

